\title{
The Frequency of Incipient Fires at the Savannah River Site
}

by

A. Blanchard

Westinghouse Savannah River Company

Savannah River Site

Aiken, South Carolina 29808

D. A. Coutts

WSMS

G. R. Morton

WSMS

T. I. Brown

WSMS

DOE Contract No. DE-AC09-96SR18500

This paper was prepared in connection with work done under the above contract number with the $U$. S. Department of Energy. By acceptance of this paper, the publisher and/or recipient acknowledges the U. S. Government's right to retain a nonexclusive, royalty-free license in and to any copyright covering this paper, along with the right to reproduce and to authorize others to reproduce all or part of the copyrighted paper. 
WSRC-TR-99-00361

\title{
The Frequency of Incipient Fires
}

\section{at the Savannah River Site (U)}

\author{
September 30, 1999
}

\author{
D. A. Coutts \\ G. R. Morton \\ T. I. Brown
}

Westinghouse Safety Management Solutions LLC 1993 South Centennial Avenue, SE

Aiken, SC 29804

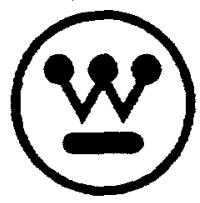




\section{DISCLAIMER}

This report was prepared as an account of work sponsored by an agency of the United States Government. Neither the United States Government nor any agency thereof, nor any of their employees, makes any warranty, express or implied, or assumes any legal liability or responsibility for the accuracy, completeness, or usefulness of any information, apparatus, product, or process disclosed, or represents that its use would not infringe privately owned rights. Reference herein to any specific commercial product, process, or service by trade name, trademark, manufacturer, or otherwise does not necessarily constitute or imply its endorsement, recommendation, or favoring by the United States Goverrment or any agency thereof. The views and opinions of authors expressed herein do not neciessarily state or reflect those of the United States Government or any agency thereof.

This report has been reproduced directly from the best available copy.

Available to DOE and DOE contractors from the Office of Scientific and Technical Information, P.O. Box 62, Oak Ridge, TN 37831; prices available from (6:15) 576-8401.

Available to the public from the National Technical Information Service, U.S. Department of Commerce; 5285 Port Royal Road, Springfield, VA 22161. 


\section{DISCLAIMER}

Portions of this document may be illegible in electronic image products. Images are produced from the best available original document. 
Key words: Fire Hazard

Fire Risk

Emergency Response

\title{
The Frequency of Incipient Fires
}

\section{at the Savannah River Site (U)}

September 30, 1999

\author{
D. A. Coutts \\ G. R. Morton \\ T. I. Brown
}

Does not contain

Unclassified Controlled Nuclear Information

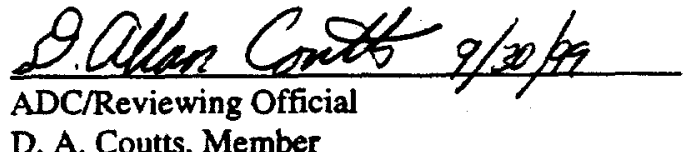

Westinghouse Safety Management Solutions LLC 1993 South Centennial Avenue, SE

Aiken, SC 29804

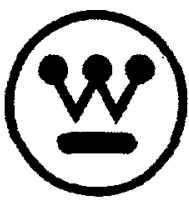


WSRC-TR-99-00361

This page intentionally blank. 
Document: WSRC-TR-99-00361

Title:

Date:

The Frequency of Incipient Fires at the Savannah River Site (U)

Authors:

September 30, 1999

D. A. Coutts G. R. Morton and T. I. Brown

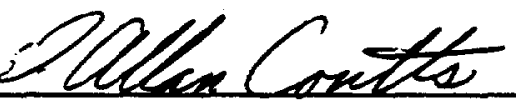

D. Allan Coutts, Member

Date

SR. Rentor

G. R. Morton, Member

lomm \&

J. I. Brovg, Member

Snown of

Date

Approvals:

DS. Cramen

$9 / 29 / 99$

D. S. Cramer, Reviewer

Date

Yon Paldeford

D. F. Paddleford, Reviewer
$9 / 29 / 99$

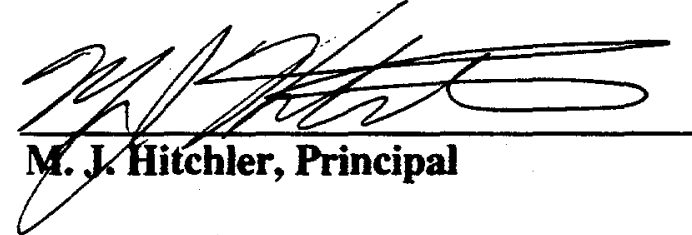

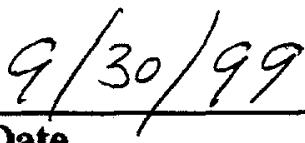

Date 
WSRC-TR-99-00361

This page intentionally blank. 


\section{Contents}

Page

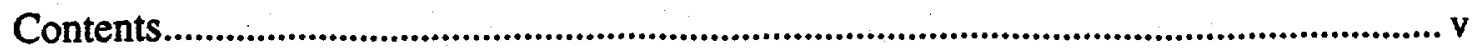

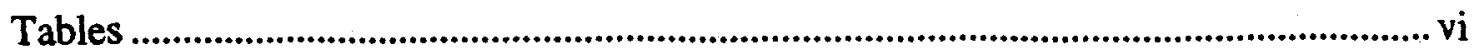

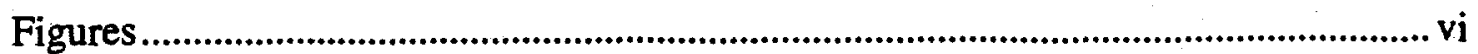

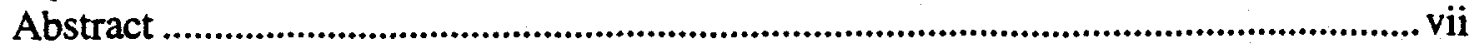

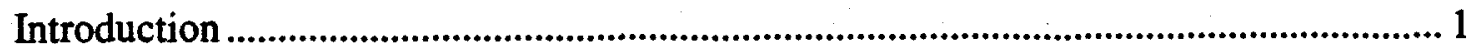

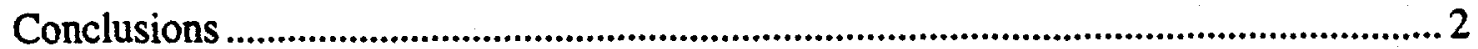

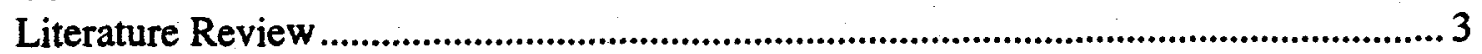

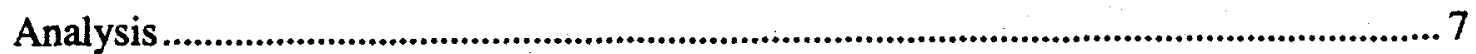

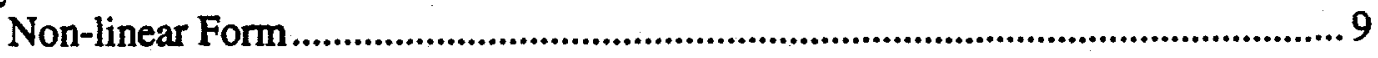

Administrative Occupancies................................................................................ 10

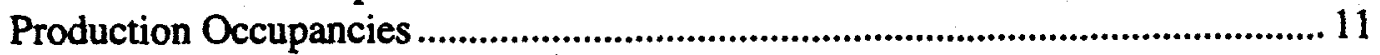

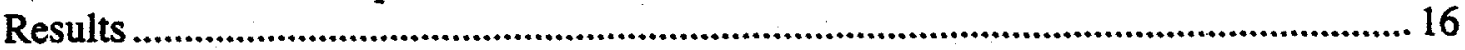

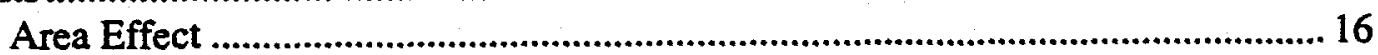

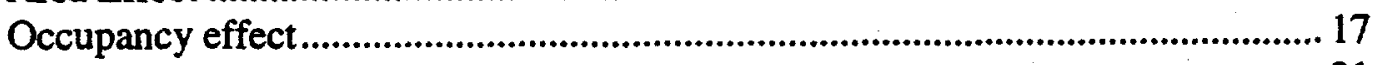

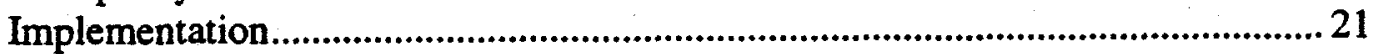

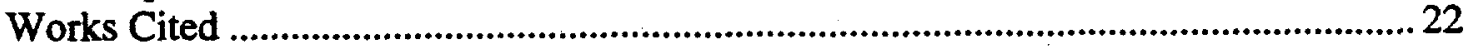

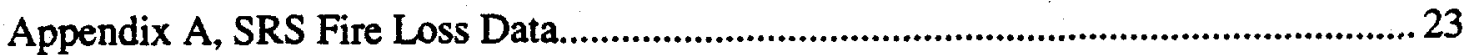

Appendix B, SRS Fire Loss Data for 1991, 1992, 1993 and 1994 .............................61

Appendix C, Background Material for Original Fire Frequency Estimator ...................65 


\section{Tables}

Table

Page

Table 1. - Original SRS incipient fire frequency results .................................................5

Table 2. -Area-based uniform fire frequency estimators.................................................5

Table 3. -Occupancy-based fire frequency estimators ............................................... 6

Table 4. - Area-based non-uniform fire frequency estimators ...........................................6

Table 5. - SRS incipient fire summaries............................................................................ 8

Table 6. - SRS uniform incipient fire frequencies by location ..................................... 9

Table 7. - Summary of SRS building fire data ……........................................................ 12

Table 8. - Fire frequency data for all SRS buildings..................................................... 12

Table 9. - Summary of SRS building fire data excluding E, G and N Areas .............. 13

Table 10. - Fire frequency data for administrative areas............................................... 13

Table 11. - Fire frequency data for production areas .................................................. 13

Table 12. - Fire frequency data for special areas ............................................................ 14

Table 13. - Fire frequency data for production areas ....................................................... 18

Table 14. - Comparison of occupancy specific correlations ............................................ 18

Table 15.-Industrial fire frequency ratios ...................................................................... 19

Table 16. - SRS production facility derivation of occupancy multiplier.......................20

Table 17. -SRS fire frequency ratios ............................................................................2 20

\section{Figures}

Figure $\quad \underline{\text { Page }}$

Figure 1, Recommended design and analysis incipient fire frequency correlations.........3

Figure 2, Fire frequency curve fits for all SRS buildings ............................................... 14

Figure 3, Fire frequency curve fits for SRS administrative areas ................................... 15

Figure 4, Fire frequency curve fit for SRS production areas ........................................... 15

Figure 5, Comparison of SRS curve fits with industrial data.......................................... 17

Figure 6, Fire frequency predictions for industrial facilities .............................................. 21 


\begin{abstract}
Fire is a significant hazard in most industrial and nuclear facilities. As such it is important that adequate safeguards be provided to ensure a responsible level of safety. In determining this level of safety it is necessary to know three key parameters. These are the frequency of the incipient fire, the probability that a fire will grow from the incipient stage to cause the potential consequence, and the potential consequences (i.e., losses) from an unwanted fire. Consequence predictions have been modeled and evaluated extensively and can be readily confirmed by comparison with historic loss records. These loss records can also provide significant insight into the probability that given a fire it grows to create a defined consequence. The other key parameter, frequency, is the focus of this report. This report determines an alternative method for estimating SRS building fire frequencies as a function of floor area to the linear method previously used.

The frequency of an incipient fire is not easily derived from existing fire loss records. This occurs because the fire loss records do not make reference to the sample population. To derive an initiating frequency both the number of events (incipient fires) and the population (number of buildings and years in service) must be known. This report documents an evaluation that estimates the frequency of incipient fires in industrial and nuclear facilities. These estimates were developed from the unique historical record that . has been maintained at the Savannah River Site.
\end{abstract}


This page intentionally blank 


\section{Introduction}

Since 1994 SRS has estimated the frequency of incipient fires based on the floor area of the building [1]. Typically, the fire frequency was considered proportional to the building area. This method also assumed that the fire frequency did not vary with building use (i.e., occupancy). In 1995 the British Standards Institute [2] published a set of incipient fire frequency correlations for industrial occupancies that were non-linear with respect to building size and included an occupancy effect. In addition, data has become available for other occupancy types [2,3]. This report reviews the newly published data, and provides an updated evaluation of the SRS fire records.

The reevaluation has demonstrated that incipient fire frequencies in Production Occupancies are non-linear with respect to building size. Thus, for very large structures the uniform frequency method (i.e., the incipient fire frequency is proportional to the size of the building) overpredicts the frequency of fire in SRS Production Occupancies, but underestimates them for smaller structures. The reevaluation also demonstrated that for SRS Administrative Occupancies the incipient fire frequency is proportional to the building area as assumed by the uniform frequency method. In addition, the reevaluation allowed the development of occupancy-based frequency multipliers. These multipliers allow the frequency estimates to account for different incipient frequencies for different : industrial occupancies.

In evaluating the SRS data and comparing it with correlations developed from other data sets the definitions for the data groups is very important. Often the data groups are not consistent between the sets. This occurs because there is no uniform classification method. In addition some data sets smear several building occupancies (i.e., uses) into one category. The SRS Administrative Occupancy is a good example of this. In practice the Administrative Occupancy is the non-Production Occupancy and thus contains office buildings, warehouses, machine shops and service facilities. Thus, any frequency results obtained for the Administrative Occupancy are smeared across several occupancy types. The confusion created by this, must be addressed by consistent use of the terms within any report and care by the reader in making comparisons with other documents. 


\section{Conclusions}

A reevaluation of the SRS Fire Department loss data has dimmonstrated that larger production buildings do in fact have a lower incipient fire frequency per unit of area. This effect can be substantial. The analysis also demonstrated that the incipient fire frequency for smaller production associated buildings exceeds values used in previous work. Thus, in terms of the new non-linear model presented below, the uniform frequency method, which is based on 33 fires $/ \mathrm{km}^{2} \cdot \mathrm{yr}$, while over-conservative for large production and industrial buildings, under-estimates the frequency for all smaller production and industrial buildings.

It was demonstrated for Administrative Occupancies that the incipient fire frequency is simply proportional to the building area as has been assumed in previous work.

The recommend design and analysis frequency predictors for use at SRS are:

Office occupancies

General storage occupancies

Production and industrial facilities

Other locations

$$
\begin{aligned}
& F=1.0 E-5 A \\
& F=3.3 E-5 A \\
& F=0.0017 \mathrm{KA} \mathrm{A}^{0.53} \\
& F=0.0027 \mathrm{~A}^{0.52}
\end{aligned}
$$

Where $\mathrm{F}$ is the fire frequency (fires/year), $\mathrm{A}$ is the building; area $\left(\mathrm{m}^{2}\right)$ and $\mathrm{K}$ is an occupancy-based multiplier:

Low potential (vault storage, reactor operations)

Nominal potential (general industry, simple operations)

High potential (laboratories, material processing facilities)
0.8

1.5

\begin{tabular}{|c|c|c|c|c|c|}
\hline \multirow[b]{2}{*}{ Building } & \multirow[b]{2}{*}{ Area, $m^{2}$} & \multirow[b]{2}{*}{ Occupancy } & \multirow[b]{2}{*}{$\mathbf{K}$} & \multicolumn{2}{|c|}{ Frequencies, fires/year } \\
\hline & & & & $\begin{array}{l}\text { original } \\
\text { value }\end{array}$ & $\begin{array}{l}\text { updated } \\
\text { value }\end{array}$ \\
\hline $105-K$ & 46000 & Production - Low potential & $\overline{0.8}$ & 1.52 & 0.40 \\
\hline $221-\mathrm{H}$ & 31000 & Production - High potential & 3.0 & 1.02 & 1.22 \\
\hline $233-\mathrm{H}$ & 3000 & Production - Nominal potential & 1.2 & 0.10 & 0.14 \\
\hline 234-H & 5000 & Production - Nominal potential & 1.2 & 0.17 & 0.19 \\
\hline 703-A & 16000 & Office & $\ldots$ & 0.53 & 0.16 \\
\hline $772-\mathrm{F}$ & 8000 & Production - High potential & 3.0 & 0.26 & 0.60 \\
\hline 773-A & 28000 & Production - High potential & 3.0 & 0.92 & 1.16 \\
\hline
\end{tabular}

Examples of the effect of these new correlations on typical buildings at SRS is presented below. Figure 1 presents a comparison of the more important recommended design and analysis frequency predictors listed above with the previous uniform frequency method. 


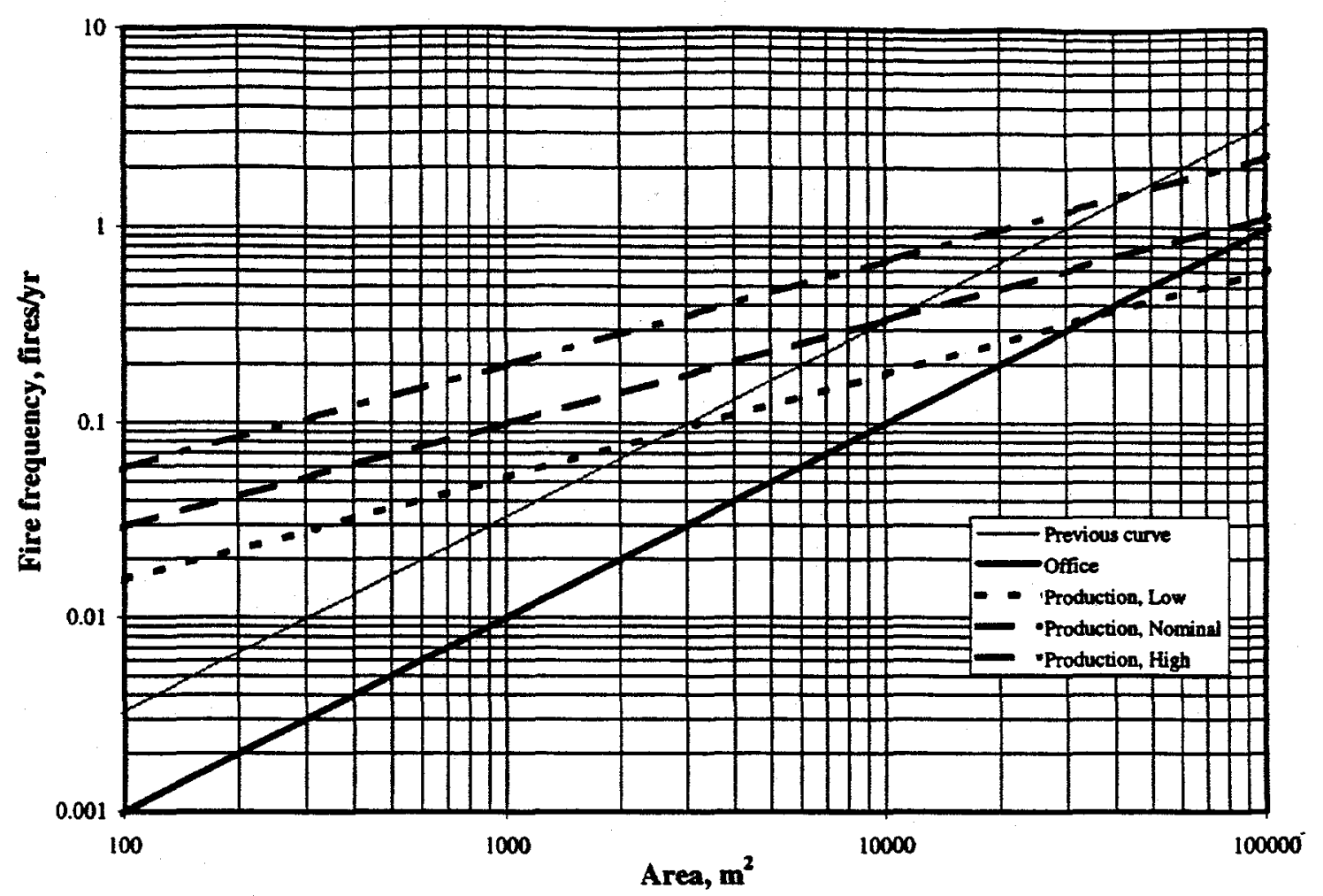

Figure 1, Recommended design and analysis incipient fire frequency correlations

More generally, the following is true for production and industrial buildings with nominal fire potential and a floor area of less than $10,000 \mathrm{~m}^{2}$, or with high fire potential and floor area of less than $45,000 \mathrm{~m}^{2}$, the previous linear estimator of 33 fires $/ \mathrm{km}^{2} \cdot y r$ underestimated the fire frequencies. 


\section{Literature Review}

For the past several years SRS has relied on a uniform incipient fire frequency value of 33 fires $/ \mathrm{km}^{2} \cdot \mathrm{yr}\left(3.3 \mathrm{E}-5 \mathrm{fires} / \mathrm{m}^{2} \cdot \mathrm{yr}\right)$ for predicting the frequency of an incipient fire [1]. This value was developed from the site-specific data [4] in Table 1 that was derived from SRS Fire Department loss records [5]. At the time that the uniform incipient fire frequency was published, based on the information available, no significant difference was identified between production and administrative building groups. The basis for the frequencies in Table 1 is data and evaluation prepared in support of the Multi-AttributeUtility model [4] that has been unpublished until this report. This basis is presented as Appendix C.

Other incipient fire frequency estimators have been published in the same form as in Table 1. These values are between one-forth and three times the SRS value and are presented in Table 2. Thus, they are within a factor of 3 or 4 of the SRS value.

Fire frequency estimators have also been published on a building basis as shown in Table 3. These values have the inherent weakness that they do not reflect the known increase in incipient fires as the building size increases. It is possible to estimate the area-based frequency from these values by assuming a nominal area. For dwellings a reasonable size estimate would be $150 \mathrm{~m}^{2}$. Thus, the area-based fire frequency would be:

(1) $F^{\prime \prime}=\frac{F}{A}=\frac{0.0030 \frac{\text { fires }}{\text { year }}}{150 \mathrm{~m}^{2}}=20 \frac{\text { fires }}{\mathrm{km}^{2} \cdot \mathrm{yr}}$

For other types of occupancies a higher average size is appropriate. Since these areas are basically conjectures with large uncertainties, they will nol: be developed further in this report.

For industrial facilities it has been shown that the fire frequency is not linearly proportional to the area [2]. The equation form is:

(2) $\mathrm{F}=\mathrm{aA}$

where the coefficient $b$ is less than unity and the frequency, $F$, is given in fires per year. Thus, the rate (or slope) of fire frequency as the building area increases falls below the relationship that the frequency is proportional to the area. That is, the incremental increase in frequency is always decreasing with increasing; area. This suggests that that the use of a single constant, such as 33 fires $/ \mathrm{km}^{2} \cdot \mathrm{yr}$, for estimating the incipient fire frequency may be over-conservative for very large buildings. 
Table 1. - Original SRS incipient fire frequency results

\begin{tabular}{|c|c|c|}
\hline Area & Occupancy & $\begin{array}{l}\text { Frequency [4] } \\
\left(\text { fires } / \mathrm{km}^{2} \cdot \mathrm{yr} \text { ) }\right.\end{array}$ \\
\hline$\overline{\mathbf{A}}$ & Administration & 18.4 \\
\hline B & Administration & 13.3 \\
\hline $700 \mathrm{C}$ & Administration & 12.7 \\
\hline $700 \mathrm{D}$ & Administration & 31.2 \\
\hline $700 F$ & Administration & 49.4 \\
\hline $700 \mathrm{H}$ & Administration & 17.4 \\
\hline $700 \mathrm{~K}$ & Administration & 6.9 \\
\hline $700 \mathrm{~L}$ & Administration & 66.0 \\
\hline $700 \mathrm{M}$ & Administration & 58.7 \\
\hline $700 \mathrm{P}$ & Administration & 40.0 \\
\hline 700R & Administration & 15.6 \\
\hline 100 & Production & 9.2 \\
\hline $400 \mathrm{D}$ & Production & 66.3 \\
\hline $200 F$ & Production & 50.5 \\
\hline $200 \mathrm{H}$ & Production & 30.4 \\
\hline $300 \mathrm{M}$ & Production & 78.7 \\
\hline $\mathbf{S}$ & Production & Not reported \\
\hline & Production & 12.8 \\
\hline \multirow[t]{6}{*}{ G,N,E } & Special & 14.1 \\
\hline & Mean & 33 \\
\hline & Standard deviation & 23 \\
\hline & Median & 24 \\
\hline & Minimum & 7 \\
\hline & Maximum & 79 \\
\hline
\end{tabular}

Table 2. - Area-based uniform fire frequency estimators

\begin{tabular}{l|c|l}
\hline \multicolumn{1}{c|}{ Occupancy } & $\begin{array}{c}\text { Frequency } \\
\text { fires/km².yr }\end{array}$ & \multicolumn{1}{c}{ Reference } \\
\hline SRS - 1994 & 33 & SRS [1] \\
Apartments & 22 & Australia [3] \\
Offices & 8.9 & Australia [3] \\
Offices & 12 & United States [2]* \\
Storage & 33 & United States [2]* \\
Public assembly & 97 & United States [2]* \\
\hline
\end{tabular}

*Attributed to F. Smith ("How successful are sprinklers?" Fire Prevention. 1982, pp. 28-35) however this citation does not present these values. 
Table 3. - Occupancy-based fire frequency estimators

\begin{tabular}{l|c|l}
\hline \multicolumn{1}{c|}{ Occupancy } & $\begin{array}{c}\text { Frequency } \\
\text { fires/building.yr }\end{array}$ & Reference \\
\hline Industrial & 0.044 & United Kingdom [2] \\
Storage & 0.013 & United Kingdom [2] \\
Offices & 0.0062 & United Kingdom [2] \\
Assembly entertainment & 0.12 & United Kingdom [2] \\
Assembly non-residential & 0.020 & United Kingdom [2] \\
Hospitals & 0.30 & United Kingdom [2] \\
Schools & 0.04 & United Kingdom [2] \\
Dwellings & 0.0030 & United Kingdom [2] \\
\hline
\end{tabular}

Table 4. - Area-based non-uniform fire frequency estimators

\begin{tabular}{l|c|c|l}
\hline \multicolumn{1}{c|}{ Occupancy } & $\mathrm{a}$ & $\mathrm{b}$ & \multicolumn{1}{c}{ Reference } \\
\hline All manufacturing & 0.0017 & 0.53 & United Kingdom [2] \\
Food, drink, tobacco & 0.0011 & 0.60 & United Kingdom [2] \\
Chemical and allied & 0.0069 & 0.46 & United Kingdom [2] \\
Mechanical engineering & 0.0001 & 0.75 & United Kingdom [2] \\
Electrical engineering & 0.0006 & 0.59 & United Kingdom [2] \\
Vehicle manufacture & 0.0001 & 0.86 & United Kingdom [2] \\
Metal goods & 0.0016 & 0.54 & United Kingdom [2] \\
Textiles & 0.0075 & 0.35 & United Kingdom [2] \\
Paper, printing, publishing & 0.00007 & 0.91 & United Kingdom [2] \\
Other manufacturing & 0.0084 & 0.41 & United Kingdom [2]
\end{tabular}




\section{Analysis}

The SRS loss data in Appendix A has been updated to include the fire history in 1991, 1992, 1993 and $1994[6,7,8,9]$. These fires are summarized in Appendix B. In addition, minor revisions to the data were made to reflect the best available data. These updates are based on interviews with site personnel, the most recent SRS Building List and analysts judgement. A sensitivity review for outliers demonstrates that these revisions had no significant effect on the final results.

Table 5 summarizes the incipient fire history for SRS from January 1958 to December 1994. The original analysis assumed that all buildings had been in service for 33 years. This neglected the construction boom that occurred in the early 1980s and had the effect of under-predicting the fire frequency. The size-year values in Table 5 present the product of the combined building size and 33 years. The size-year data for the update are based on the individual building size and the actual number of years that a building was in service. This approach avoids the error of assuming that all of the buildings were built in the same year. Appendix A presents all of the data used in this analysis.

The area-specific uniform fire frequencies are presented in Table 6. With the exception of A-Area and 400D, the frequencies calculated using the data base information replicate, the original published work within expected roundoff error. In the original work Building 773-A was excluded as an outlier (28,267 $\mathrm{m}^{2}$ of area and 96 fires). If this is accounted for, the replicated uniform frequency for A-Area would be:

$$
\text { (3) } \quad F^{\prime \prime}=\frac{169 \text { fires }-96 \text { fires }}{\left(148,432 \mathrm{~m}^{2}-28,267 \mathrm{~m}^{2}\right)(33 \text { years })}=18.4 \frac{\text { fires }}{\mathrm{km}^{2} \cdot \mathrm{yr}}
$$

The updated results have a greater variability than the original results. The uniform-area frequencies range from 9 to 110 fires $/ \mathrm{km}^{2} \cdot \mathrm{yr}$. The mean value increased from 33 to 50 fires $/ \mathrm{km}^{2} \cdot \mathrm{yr}$. The median value increased from 23 to 28 fires $/ \mathrm{km}^{2} \cdot \mathrm{yr}$. 
WSRC-TR-99-00361

Table 5. - SRS incipient fire summaries

\begin{tabular}{|c|c|c|c|c|c|c|c|}
\hline \multirow[b]{2}{*}{ Area } & \multirow[b]{2}{*}{ Occupancy } & \multicolumn{2}{|c|}{$\begin{array}{c}\text { Combined } \\
\text { building size, } \\
\mathrm{m}^{2}\end{array}$} & \multicolumn{2}{|c|}{$\begin{array}{c}\text { Size-years } \\
\mathrm{m}^{2} \cdot \mathrm{yr}\end{array}$} & \multicolumn{2}{|c|}{ Number of fires } \\
\hline & & Original & Updated & Original & Updated & Original & Updated \\
\hline $\bar{A}$ & Administration & 148432 & 149529 & 4898256 & 4169083 & 169 & 186 \\
\hline B & Administration & 6856 & 7285 & 226248 & 55783 & 3 & 5 \\
\hline $700 \mathrm{C}$ & Administration & 11972 & 12783 & 395076 & 211919 & 5 & 8 \\
\hline $700 \mathrm{D}$ & Administration & 4861 & 4861 & 160413 & 181965 & 5 & 5 \\
\hline $700 \mathrm{~F}$ & Administration & 25730 & 25726 & 849090 & 746506 & 42 & 44 \\
\hline $700 \mathrm{H}$ & Administration & 12150 & 11107 & 400950 & 185544 & 7 & 12 \\
\hline $700 \mathrm{~K}$ & Administration & 4380 & 4537 & 144540 & 91054 & 1 & 3 \\
\hline $700 \mathrm{~L}$ & Administration & 1835 & 1835 & 60555 & 60010 & 4 & 4 \\
\hline $700 \mathrm{M}$ & Administration & 2584 & 2591 & 85272 & 49194 & 5 & 5 \\
\hline $700 \mathrm{P}$ & Administration & 2268 & 2269 & 74844 & 79243 & 3 & 3 \\
\hline $700 \mathbf{R}$ & Administration & 1939 & 1940 & 63987 & 63696 & 1 & 1 \\
\hline 100 & Production & 311900 & 311902 & 10292700 & 11712194 & 95 & 106 \\
\hline 400D & Production & 34291 & 34288 & 1131603 & 1239614 & 75 & 85 \\
\hline $200 \mathrm{~F}$ & Production & 70227 & 72170 & 2317491 & 2025392 & 117 & 131 \\
\hline $200 \mathrm{H}$ & Production & 61986 & 69851 & 2045538 & 2123424 & 62 & 77 \\
\hline $300 \mathrm{M}$ & Production & 31483 & 31481 & 1038939 & 800995 & 82 & 87 \\
\hline $\mathbf{S}$ & Production & $\cdots$ & 53380 & $\cdots$ & 201365 & $\cdots$ & 6 \\
\hline $\mathrm{T}$ & Production & 14264 & 14264 & 470712 & 232069 & 6 & 6 \\
\hline \multirow[t]{2}{*}{ G,N,E } & Special & 53935 & 57440 & 1779855 & 1367896 & 25 & 39 \\
\hline & Sum & 801093 & 869239 & 26436069 & 25596946 & 707 & 813 \\
\hline
\end{tabular}


Table 6. - SRS uniform incipient fire frequencies by location

\begin{tabular}{|c|c|c|c|c|}
\hline \multirow[b]{3}{*}{ Area } & \multirow[b]{3}{*}{ Occupancy } & \multicolumn{3}{|c|}{ Frequency $\left(\right.$ fires $/ \mathrm{km}^{2} \cdot \mathrm{yr}$ ) } \\
\hline & & \multicolumn{2}{|c|}{ Original } & \multirow{2}{*}{$\begin{array}{c}\text { Updated } \\
\text { calculated }\end{array}$} \\
\hline & & reported & calculated & \\
\hline$\overline{\mathbf{A}}$ & Administration & 18.4 & 34.5 & $\overline{44.6}$ \\
\hline B & Administration & 13.3 & 13.3 & 89.6 \\
\hline $700 \mathrm{C}$ & Administration & 12.7 & 12.7 & 37.8 \\
\hline $700 \mathrm{D}$ & Administration & 31.2 & 31.2 & 27.5 \\
\hline $700 \mathrm{~F}$ & Administration & 49.4 & 49.5 & 58.9 \\
\hline $700 \mathrm{H}$ & Administration & 17.4 & 17.5 & 64.7 \\
\hline $700 \mathrm{~K}$ & Administration & 6.9 & 6.9 & 32.9 \\
\hline $700 \mathrm{~L}$ & Administration & 66.0 & 66.1 & 66.7 \\
\hline $700 \mathrm{M}$ & Administration & 58.7 & 58.6 & 101.6 \\
\hline $700 \mathrm{P}$ & Administration & 40.0 & 40.1 & 37.9 \\
\hline 700R & Administration & 15.6 & 15.6 & 15.7 \\
\hline 100 & Production & 9.2 & 9.2 & 9.1 \\
\hline $400 \mathrm{D}$ & Production & 66.3 & 64.5 & 67.0 \\
\hline $200 \mathrm{~F}$ & Production & 50.5 & 50.5 & 64.7 \\
\hline $200 \mathrm{H}$ & Production & 30.4 & 30.3 & 36.3 \\
\hline $300 \mathrm{M}$ & Production & 78.7 & 78.9 & 108.6 \\
\hline S & Production & $\ldots$ & $\cdots$ & 29.8 \\
\hline $\mathrm{T}$ & Production & 12.8 & 12.7 & 25.9 \\
\hline $\mathrm{G}, \mathrm{N}, \mathrm{E}$ & Special & 14.1 & 14.0 & 28.5 \\
\hline \multicolumn{2}{|l|}{ Mean } & 33 & 34 & 50 \\
\hline \multicolumn{2}{|c|}{ Std. Deviation } & 23 & 23 & 28 \\
\hline \multicolumn{2}{|c|}{ Median } & 24 & 31 & 38 \\
\hline \multicolumn{2}{|c|}{ Minimum } & 7 & 7 & 9 \\
\hline \multicolumn{2}{|c|}{ Maximum } & 79 & 79 & 110 \\
\hline
\end{tabular}

\section{Non-linear Form}

The primary intent of the study presented in this report was to evaluate the non-linear fire frequency estimator, $F$. As was discussed above when analyzing fire frequency data it is necessary to normalize the results in terms of area-years. Thus, the data becomes fires per year per unit area (fires $/ \mathrm{km}^{2} \cdot \mathrm{yr}$ ) rather than fires per year as given in Equation (2). To accommodate this, a normalized fire frequency equation will be used for analysis purposes. This normalized frequency is:

$$
\text { (4) } F^{\infty}=\alpha A^{\beta}=a b A^{b-1}
$$

Thus, to transform the results developed using Equation (4) into the design form of Equation (2):

$$
\text { (5) } a=\frac{\alpha}{(\beta+1)}
$$


The median size of the buildings listed in Appendix A is $89 \mathrm{~m}^{2}$. Twenty-five percent of the buildings are smaller than $29 \mathrm{~m}^{2}, 75$ percent of the buildings are smaller than $280 \mathrm{~m}^{2}$, and 90 percent are smaller than $1100 \mathrm{~m}^{2}$. The largest building is $69677 \mathrm{~m}^{2}$. This suggests the following logical groupings based on size:

$\begin{array}{ll}\text { Very small } & A \leq 30 \mathrm{~m}^{2} \\ \text { Small } & 30<A \leq 90 \mathrm{~m}^{2} \\ \text { Medium } & 90<A \leq 300 \mathrm{~m}^{2} \\ \text { Large } & 300<A \leq 1000 \mathrm{~m}^{2}\end{array}$

In addition, the largest building that did not have a fire had an area of slightly less than $5000 \mathrm{~m}^{2}$. Thus, for buildings greater than $5000 \mathrm{~m}^{2}$ it is poissible to explicitly calculate the fire frequency. For buildings smaller than $5000 \mathrm{~m}^{2}$ the data is grouped as shown in Table 7. These data are plotted in Figure 2 along with the individual building frequencies from Table 8. To account for the unequal area-years of history the curve fit was weighted using the area-years value. The weighted curve fit for all of the SRS data is:

(7) $F^{\circ}=630 \mathrm{~A}^{-0.39}$

On inspection of the E, G, N Areas data, which is treated as a special occupancy, it is uncertain if the information is complete. Thus, a curve fit was generated excluding this data. Table 9 provides the summary of the SRS building fire data after the exclusion of $\mathrm{E}, \mathrm{G}$, and $\mathrm{N}$ Areas. The resulting weighted curve fit is:

(8) $F^{\prime \prime}=910 \mathrm{~A}^{-0.42}$

The original study excluded Building 773-A as an outlier. The data for Buildings 105-L, 221-F and 313-M could also be excluded based on the same logic. If these data and the information for $\mathrm{E}, \mathrm{G}$ and $\mathrm{N}$ Areas area excluded, the weighted curve fit is:

(9) $F^{\prime}=850 \mathrm{~A}^{-0.43}$

These three curve fits are shown in Figure 2. There is little: difference in the results. Since the "outliers" have little effect it is recommended that the curve fit that excludes $E$, $\mathrm{G}$ and $\mathrm{N}$ Area data be the fit of choice to cover all SRS buildings (i.e., Equation (8)). When transformed back to the design equation form, Equation (8) becomes:

(10) $F=0.0016 A^{0.58}$

\section{Administrative Occupancies:}

The analysis for all building data can be repeated for both Administrative and Production Occupancies. Table 10 provides a summary of the Administrative Occupancy fire loss data that has been combined with the Administrative Occupancy data in Table 8 and plotted in Figure 3. Two weighted curve fits have been presented. The first includes all 
data; the second excludes the data from Buildings 772-F and 773-A. Both of these buildings are actually laboratories, rather than the more common Administrative Occupancies (e.g., office buildings, maintenance shops, warehouses). The respective weighted curve fits are:

(11) $F^{\prime \prime}=19 A^{0.085}$

(12) $\mathrm{F}^{\prime \prime}=150 \mathrm{~A}^{-0.21}$

The first fit, which includes data from the two laboratory buildings, has a positive slope. This is the opposite of that expected. After excluding the two laboratory buildings the slope becomes negative. The present data does not suggest a strong variation with increasing area. Thus, it is recommended that weighted mean (excluding 772-F and 773A) be used for fire frequency estimates for Administrative Occupancies. This mean value is 35 fires $/ \mathrm{km}^{2} \cdot \mathrm{yr}$.

\section{Production Occupancies}

Table 11 provides a summary of the Production Occupancy fire loss data. (Information on Special Occupancies is shown in Table 12 to allow comparison.) This data has been combined with the Production Occupancy data in Table 8 and plotted in Figure 4. Two weighted curve fits have been presented. The first includes all data; the second excludes the data from Buildings 105-L, 221-F and 313-M. The respective weighted curve fits are:

$$
\begin{aligned}
& F^{\prime}=1400 A^{-0.48} \\
& F^{\prime \prime}=950 A^{-0.44}
\end{aligned}
$$

These curves are very similar and since there is no strong evidence to exclude data from any of the buildings, Equation (13) is the recommended curve fit for Production Occupancies. When transformed back to the design equation form, Equation (13) becomes:

(15) $\mathrm{F}=0.0027 \mathrm{~A}^{0.52}$ 
Table 7. - Summary of SRS building fire data

\begin{tabular}{|c|c|c|c|c|c|c|}
\hline \multirow[t]{2}{*}{ Size } & \multirow{2}{*}{$\begin{array}{c}\begin{array}{c}\text { Number } \\
\text { of } \\
\text { buildings }\end{array} \\
\end{array}$} & \multirow{2}{*}{$\begin{array}{c}\text { Number of } \\
\text { fires }\end{array}$} & \multicolumn{2}{|c|}{ Area, $\mathrm{m}^{2}$} & \multirow{2}{*}{$\begin{array}{c}\text { Area-years } \\
\mathrm{m}^{2} \cdot \mathrm{yr}\end{array}$} & \multirow{2}{*}{$\begin{array}{c}F^{n} \\
\text { fires } / \mathrm{km}^{2} \cdot \mathrm{yr}\end{array}$} \\
\hline & & & Mean & Sum & & \\
\hline$\leq 30$ & 303 & 56 & 14 & 4276 & 72073 & 777 \\
\hline 30 to 90 & 295 & 15 & 63 & 18521 & 229978 & 65 \\
\hline 90 to 300 & 295 & 91 & 160 & $471: 4$ & 604614 & 151 \\
\hline 300 to 1000 & 145 & 73 & 560 & 81478 & 2017036 & 36 \\
\hline 1000 to 5000 & 115 & 128 & 1900 & $2223: 5$ & 5031352 & 25 \\
\hline$>5000$ & 24 & 450 & 21000 & 495535 & 17641893 & 25 \\
\hline Sums & 1177 & 813 & & 869239 & 25596946 & \\
\hline
\end{tabular}

Table 8. - Fire frequency data for all SRS buildings

\begin{tabular}{l|l|r|r|c|c}
\hline Occupancy & Building & $\begin{array}{c}\text { Average size } \\
\mathrm{m}^{2}\end{array}$ & $\begin{array}{c}\text { Area-years } \\
\mathrm{m}^{2} \cdot \mathrm{yr}\end{array}$ & $\begin{array}{c}\text { Number } \\
\text { of fires }\end{array}$ & $\begin{array}{c}\mathrm{F}^{n} \\
\text { fires/km } \mathrm{km}^{2} \cdot \mathrm{yr}\end{array}$ \\
\hline Production & $105-000 \mathrm{C}$ & 46452 & 1718724 & 18 & 10 \\
Production & $105-000 \mathrm{~K}$ & 46452 & 1718724 & 15 & 9 \\
Production & $184-000 \mathrm{~K}$ & 7897 & 292189 & 11 & 2 \\
Production & $105-000 \mathrm{~L}$ & 46452 & 1718724 & 3 & 6 \\
Production & $184-000 \mathrm{~L}$ & 7897 & 292189 & 3 & 4 \\
Production & $105-000 \mathrm{P}$ & 69677 & 2578049 & 15 & 38 \\
Production & $184-000 \mathrm{P}$ & 7897 & 292189 & 12 & 10 \\
Production & $105-000 \mathrm{R}$ & 46452 & 1904532 & 8 & 41 \\
Production & $184-000 \mathrm{R}$ & 7897 & 292189 & 3 & 10 \\
Production & $210-000 \mathrm{~S}$ & 8696 & 34784 & 1 & 29 \\
Production & $221-000 \mathrm{~S}$ & 17782 & 71128 & 2 & 77 \\
Administration & $772-000 \mathrm{~F}$ & 7785 & 311400 & 24 & 27 \\
Production & $484-000 \mathrm{D}$ & 23226 & 859362 & 10 & 28 \\
Production & $313-000 \mathrm{M}$ & 5315 & 223230 & 32 & 45 \\
Production & $320-000 \mathrm{M}$ & 5880 & 217560 & 20 & 31 \\
Production & $321-000 \mathrm{M}$ & 5209 & 192733 & 17 & 48 \\
Production & $221-000 \mathrm{H}$ & 30621 & 1224840 & 33 & 143 \\
Production & $232-000 \mathrm{H}$ & 5130 & 200070 & 9 & 92 \\
Production & $234-000 \mathrm{H}$ & 5331 & 191916 & 6 & 88 \\
Production & $221-000 \mathrm{~F}$ & 30632 & 1225280 & 94 & 12 \\
Production & $247-000 \mathrm{~F}$ & 13006 & 104048 & 5 & 16 \\
Administration & $703-000 \mathrm{~A}$ & 16444 & 608428 & 10 & 77 \\
Administration & $773-000 \mathrm{~A}$ & 28267 & 1158947 & 96 & 83 \\
Administration & $777-010 \mathrm{~A}$ & 5138 & 210658 & 3 & 14 \\
\hline & Sums & 495535 & 17641893 & 450 & \\
\hline
\end{tabular}


Table 9. - Summary of SRS building fire data excluding E, G and N Areas

\begin{tabular}{l|c|r|r|r|r|r}
\hline \multirow{2}{*}{ Size } & \multirow{2}{*}{$\begin{array}{c}\text { Number } \\
\text { of }\end{array}$} & \multirow{2}{*}{$\begin{array}{c}\text { Number of } \\
\text { buildings }\end{array}$} & \multicolumn{2}{|c|}{ Area, $\mathrm{m}^{2}$} & \multirow{2}{*}{$\begin{array}{c}\text { Area-years } \\
\text { fires }\end{array}$} & \multirow{2}{*}{$\begin{array}{c}\mathrm{F}^{\prime \prime} \\
\text { fires } / \mathrm{km}^{2} \cdot \mathrm{yr}\end{array}$} \\
\hline$\leq 30$ & 239 & 38 & 16 & 3706 & 62800 & 605 \\
30 to 90 & 262 & 12 & 63 & 16627 & 198880 & 60 \\
90 to 300 & 274 & 89 & 159 & 43527 & 539444 & 165 \\
300 to 1000 & 114 & 70 & 555 & 63307 & 1621503 & 43 \\
1000 to 5000 & 94 & 115 & 2012 & 189097 & 4164530 & 28 \\
$>5000$ & 24 & 450 & 20647 & 495535 & 17641893 & 26 \\
\hline Sums & 1007 & 774 & & 811799 & 24229050 & \\
\hline
\end{tabular}

Table 10. - Fire frequency data for administrative areas

\begin{tabular}{|c|c|c|c|c|c|c|}
\hline \multirow[t]{2}{*}{ Size } & \multirow{2}{*}{\begin{tabular}{|c|}
$\begin{array}{c}\text { Number } \\
\text { of } \\
\text { buildings }\end{array}$ \\
\end{tabular}} & \multirow{2}{*}{$\begin{array}{c}\text { Number of } \\
\text { fires }\end{array}$} & \multicolumn{2}{|c|}{ Area, $\mathrm{m}^{2}$} & \multirow{2}{*}{$\begin{array}{c}\text { Area-years } \\
\mathrm{m}^{2} \cdot \mathrm{yr}\end{array}$} & \multirow{2}{*}{$\begin{array}{c}F^{\prime \prime} \\
\text { fires } / \mathrm{km}^{2} \cdot \mathrm{yr}\end{array}$} \\
\hline & & & Mean & Sum & & \\
\hline$\leq 30$ & 91 & 24 & 15 & 1405 & 26994 & 889 \\
\hline 30 to & 112 & 2 & 66 & 7338 & 83826 & 24 \\
\hline 90 to 300 & 149 & 8 & 161 & 23978 & 305676 & 26 \\
\hline 300 to 1000 & 38 & 26 & 505 & 19175 & 496809 & 52 \\
\hline 1000 to 5000 & 55 & 83 & 2090 & 114933 & 2691259 & 31 \\
\hline Sums & 445 & 143 & & 166829 & 3604564 & \\
\hline
\end{tabular}

Table 11. - Fire frequency data for production areas

\begin{tabular}{|c|c|c|c|c|c|c|}
\hline \multirow{2}{*}{ Size } & \multirow{2}{*}{$\begin{array}{c}\text { Number } \\
\text { of } \\
\text { buildings }\end{array}$} & \multirow{2}{*}{$\begin{array}{c}\text { Number of } \\
\text { fires }\end{array}$} & \multicolumn{2}{|c|}{ Area, $\mathrm{m}^{2}$} & \multirow{2}{*}{$\begin{array}{c}\text { Area-years } \\
\mathrm{m}^{2} \cdot \mathrm{yr}\end{array}$} & \multirow{2}{*}{$\begin{array}{c}F^{\prime \prime} \\
\text { fires } / \mathrm{km}^{2} \cdot \mathrm{yr}\end{array}$} \\
\hline & & & Mean & Sum & & \\
\hline$\leq 30$ & 148 & 14 & 16 & 2301 & 35806 & 391 \\
\hline 30 to 90 & 150 & 10 & 62 & 9289 & 115054 & 87 \\
\hline 90 to 300 & 125 & 81 & 156 & 19549 & 233768 & 346 \\
\hline 300 to 1000 & 76 & 44 & 581 & 44132 & 1124694 & 39 \\
\hline 1000 to 5000 & 39 & 32 & 1902 & 74164 & 1473271 & 22 \\
\hline$\overline{\text { Sums }}$ & 538 & 181 & & 149435 & 2982593 & \\
\hline
\end{tabular}


Table 12. - Fire frequency data for special areas

\begin{tabular}{|c|c|c|c|c|c|c|}
\hline \multirow[t]{2}{*}{ Size } & \multirow{2}{*}{$\begin{array}{c}\text { Number } \\
\text { of } \\
\text { buildings }\end{array}$} & \multirow{2}{*}{$\begin{array}{c}\text { Number of } \\
\text { fires }\end{array}$} & \multicolumn{2}{|c|}{ Area, $\mathrm{m}^{2}$} & \multirow{2}{*}{$\begin{array}{c}\text { Area-years } \\
\mathrm{m}^{2} \cdot \mathrm{yr}\end{array}$} & \multirow{2}{*}{$\begin{array}{c}F^{\prime \prime} \\
\text { fires } / \mathrm{km}^{2} \cdot \mathrm{yr}\end{array}$} \\
\hline & & & Mean & Sum & & \\
\hline$\leq 30$ & 64 & 18 & 9 & 570 & 9273 & 1900 \\
\hline 30 to 9 & 33 & 3 & 57 & 189 & 310 & 97 \\
\hline 90 to 300 & 21 & 2 & 171 & 3587 & 65170 & 31 \\
\hline 300 to 1000 & 31 & 3 & 586 & 18171 & 395533 & 8 \\
\hline 1000 to 5000 & 21 & 13 & 1582 & 33218 & 866822 & 15 \\
\hline$\overline{\text { Sums }}$ & 170 & 39 & & 57440 & 1367896 & \\
\hline
\end{tabular}

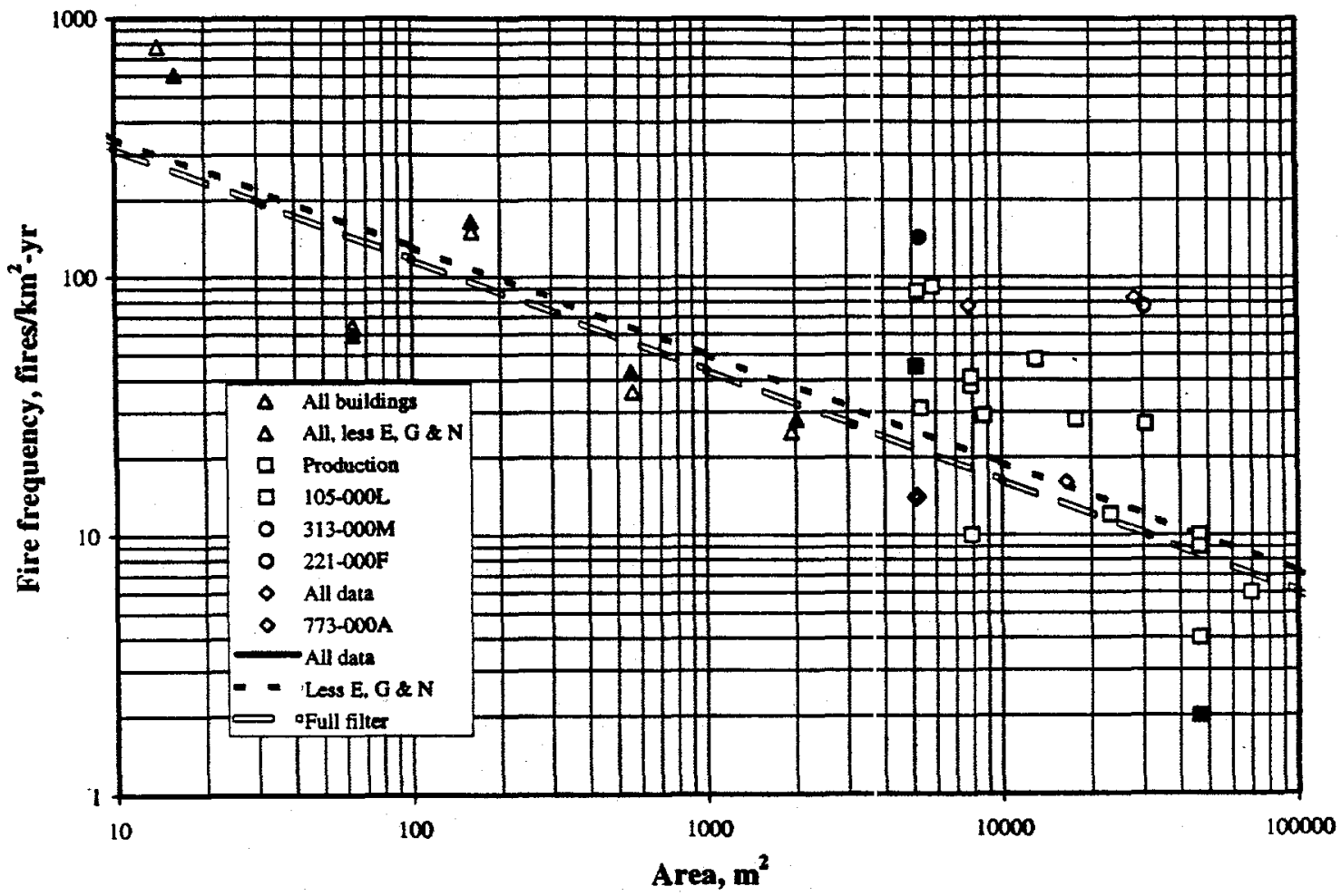

Figure 2, Fire frequency curve fits for all SRS building; 


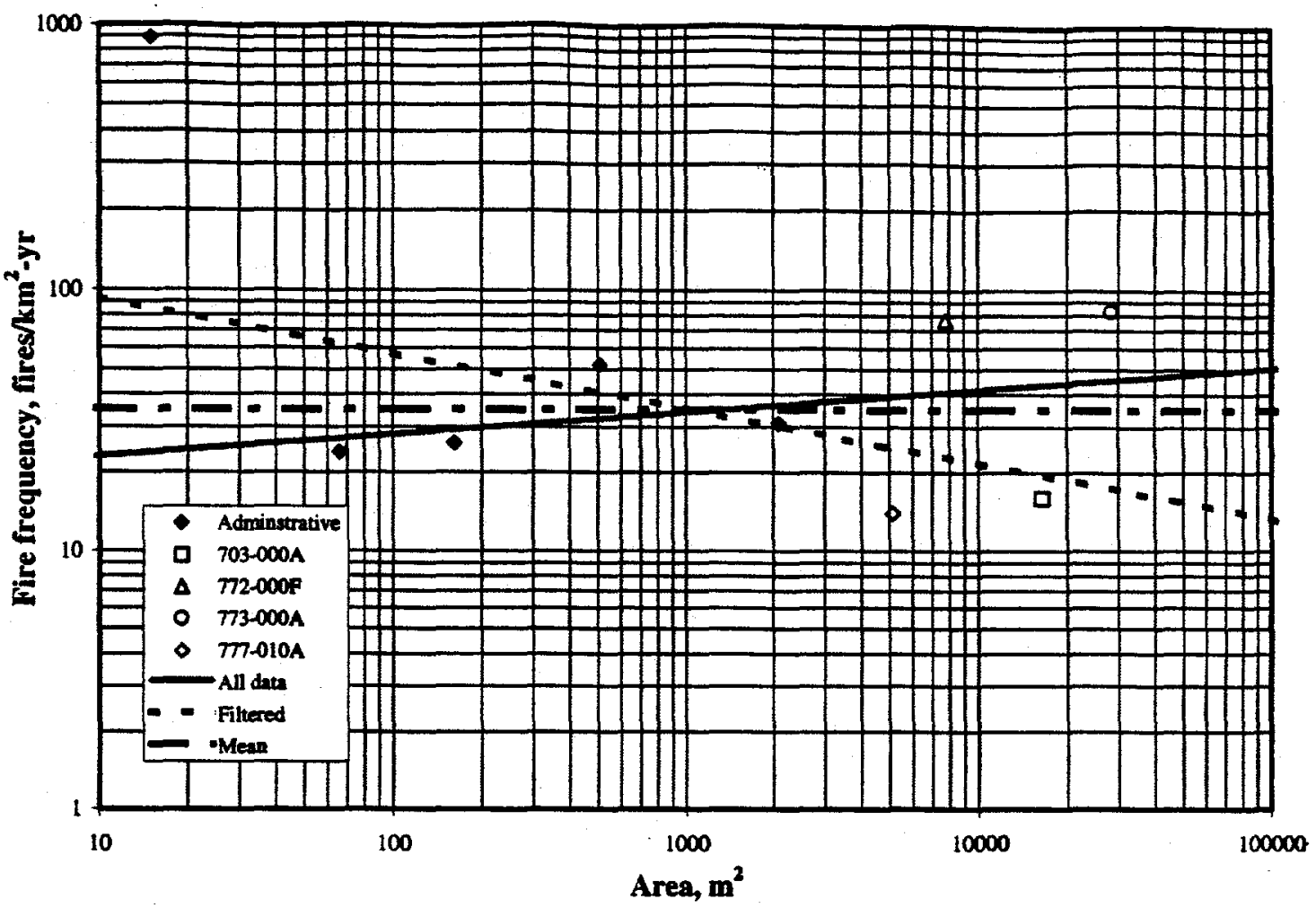

Figure 3, Fire frequency curve fits for SRS administrative areas

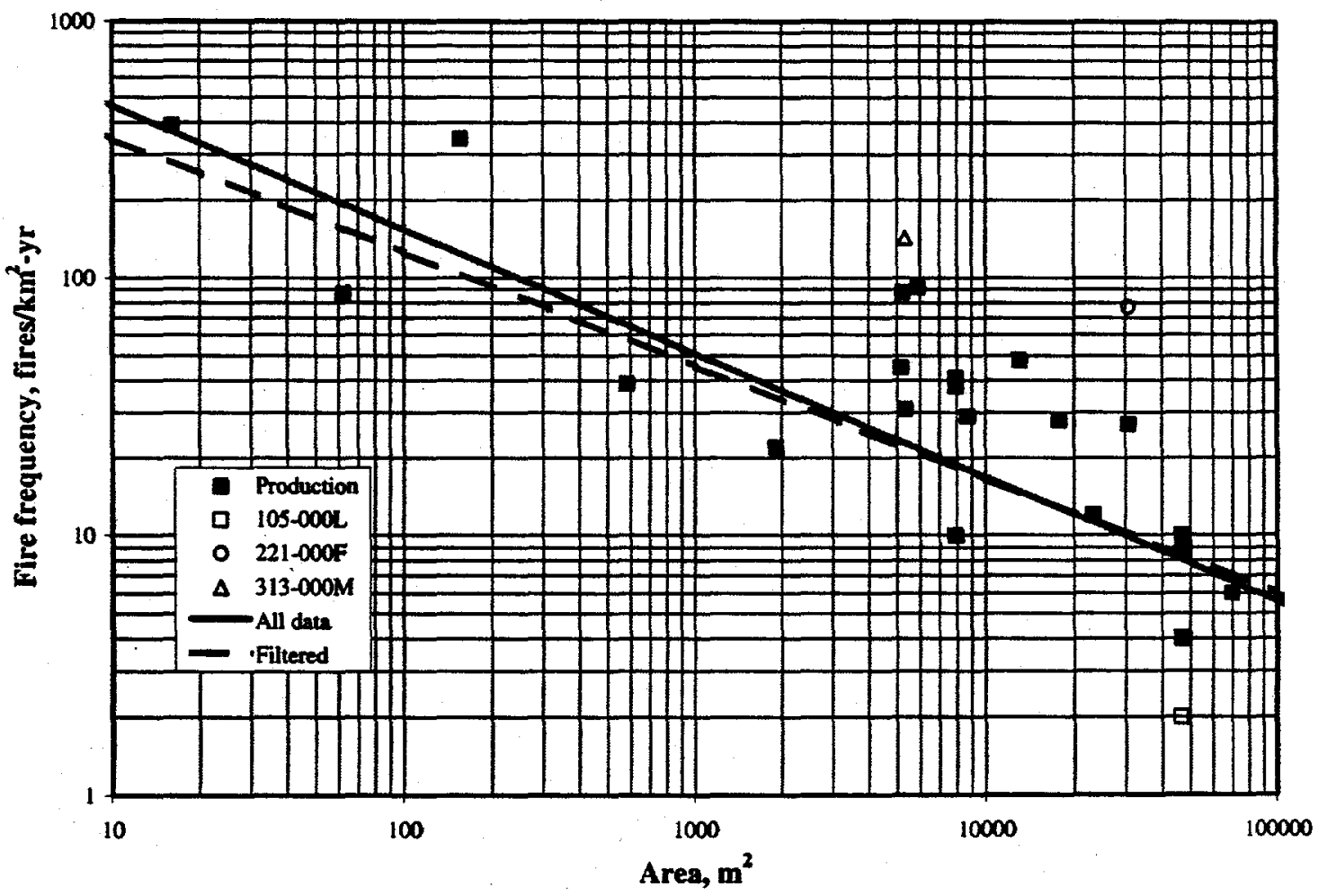

Figure 4, Fire frequency curve fit for SRS production areas 


\section{Results}

The reanalysis has demonstrated that there is a size effect for Production Facilities. This effect can be significant. The effect was not observed for Administrative Facilities. For Administrative Facilities it appears that an area-based proportional constant is all that is necessary to accurately estimate the incipient fire frequency.

The basic correlations developed from the SRS data are:

$$
\begin{array}{ll}
\text { Administrative facilities } & \mathrm{F}=3.5 \mathrm{E}-5 \mathrm{~A} . \\
\text { Production facilities } & \mathrm{F}=0.0027 \mathrm{~A}^{0.52}
\end{array}
$$

Where $\mathrm{F}$ is the fire frequency in fires per year and $\mathrm{A}$ is the building area in $\mathrm{m}^{2}$.

\section{Area Effect}

Figure 5 shows how well the SRS Production Curve, Equation (13), parallels with industrial data. The SRS curve is slightly higher than the all-manufacturing correlation and slightly below the correlation for chemical and allied processes. This would be expected since SRS production facilities represent a blend of general industry and nuclear facilities. Often these nuclear facilities are very similar to those used in the chemical industry.

Figure 5 also demonstrates the SRS all-building curve. This curve does not parallel the all manufacturing correlation. This is expected since the SRS all-building curve contains a significant portion of administrative facilities, which have been shown to be better represented using a uniform frequency estimator.

Direct comparison between the SRS Administrative Occupancy results and published literature is not readily possible. SRS Administrative Occupancies are a blend of offices, laboratories, repair and storage facilities. The published estimator for offices ranges from 8.9 to 13 fires $/ \mathrm{km}^{2}$.yr. This range is lower than the SRS estimator, which is 35 fires $/ \mathrm{km}^{2}$.yr. Figure 5 graphically demonstrates that the frequency of fires in typical industrial facilities ranges from 10 to 100 fires $/ \mathrm{km}^{2} \cdot \mathrm{yr}$ and can exceed 200 fires $/ \mathrm{km}^{2} \cdot \mathrm{yr}$ for small chemical and allied processes. Thus, it is expected that the SRS Administrative Occupancy result is higher than the published values for offices.

It is possible to generalize the SRS results to compare it with published data. To accomplish this requires the following thought experiment. Assume that one-third of the Administrative Occupancies at SRS are offices or similar coccupancies with an incipient fire frequency of 10 fires $/ \mathrm{km}^{2}$.yr. The remaining two-thirds are split equally between typical industrial and storage facilities and higher potential storage facilities. The frequency estimates for these occupancies are 33 and $80 \mathrm{files} / \mathrm{km}^{2} \cdot \mathrm{yr}$. The net effect would be an overall frequency of: 
(16)

$$
F^{\prime}=\frac{10 \frac{\text { fires }}{\mathrm{km}^{2} \cdot \mathrm{yr}}}{3}+\frac{33 \frac{\text { fires }}{\mathrm{km}^{2} \cdot \mathrm{yr}}}{3}+\frac{80 \frac{\text { fires }}{\mathrm{km}^{2} \cdot \mathrm{yr}}}{3}=41 \frac{\text { fires }}{\mathrm{km}^{2} \cdot \mathrm{yr}}
$$

This result exceeds the 35 fires $/ \mathrm{km}^{2} \cdot \mathrm{yr}$, but is less then the $46 \mathrm{fires} / \mathrm{km}^{2} \cdot \mathrm{yr}$ that is the Administrative Occupancy result prior to excluding Buildings 772-F and 773-A. Thus, the SRS Administrative Occupancy results do compare favorably with published values.

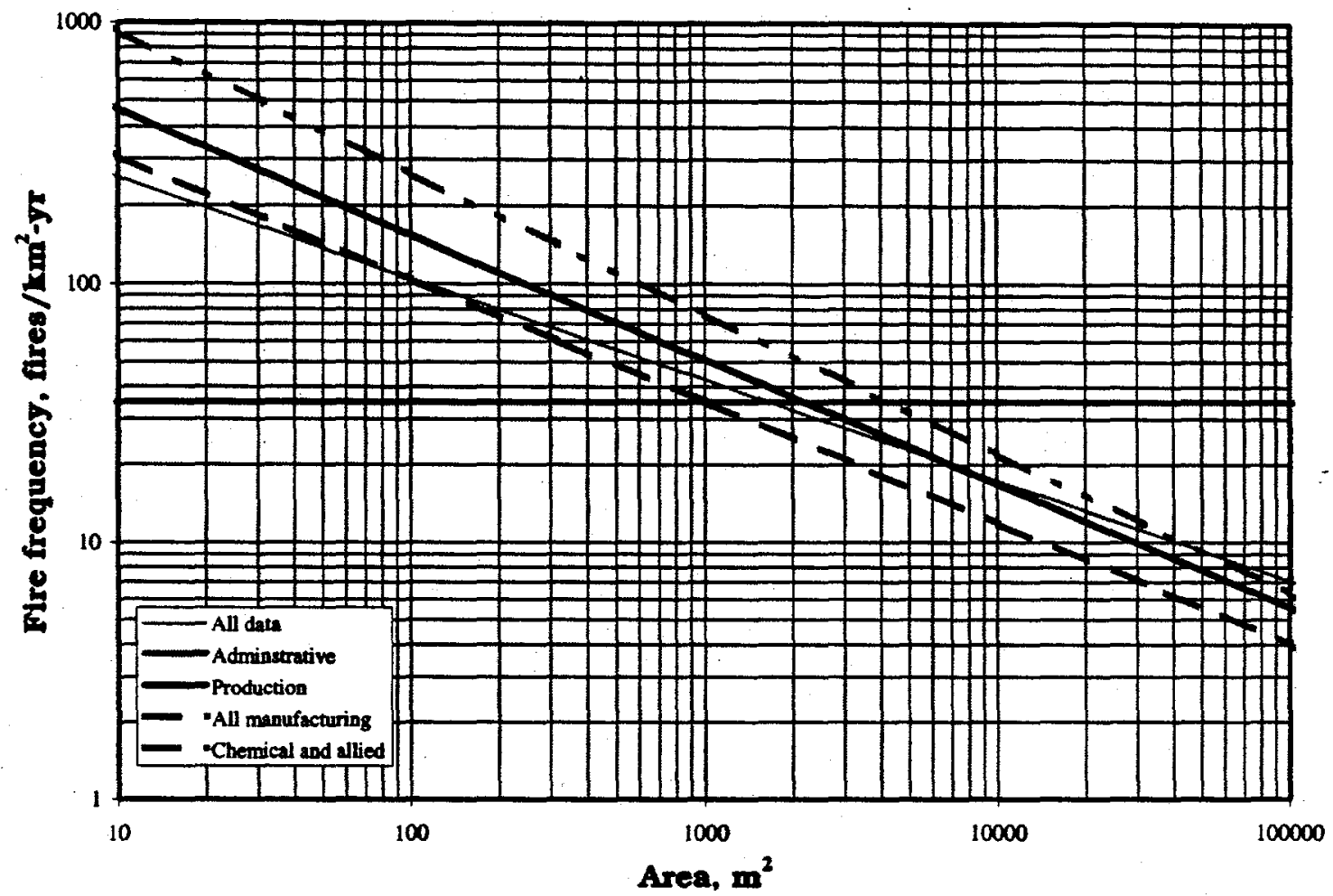

Figure 5, Comparison of SRS curve fits with industrial data

\section{Occupancy effect}

The fire frequency estimators presented in Table 4 can be recast using the form for Equation (4). This is presented in Table 13. For typical building sizes the normalized incipient fire frequencies all show a decrease in the normalized value as the building size increases. This is shown in Figure 6. For many occupancies the equation slopes are almost identical. Where the slopes are similar it is reasonable to represent the area effect with a single equation and account for the occupancy using a multiplier, $\mathrm{K}$.

(17) $\mathrm{F}=\mathrm{a} \cdot \mathrm{K} \cdot \mathrm{A}^{\mathrm{b}}$ 
Table 13. - Fire frequency data for production areas

\begin{tabular}{l|r|r}
\hline \multicolumn{1}{c|}{ Building size $\left(\mathrm{m}^{2}\right)$} & $\alpha$ & $\beta$ \\
\hline SRS new & 610 & -0.39 \\
All manufacturing & 901 & -0.47 \\
Food, drink, tobacco & 660 & -0.40 \\
Chemical and allied & 3174 & -0.54 \\
Mechanical engineering & 75 & -0.25 \\
Electrical engineering & 354 & -0.41 \\
Vehicle manufacture & 86 & -0.14 \\
Metal goods & 864 & -0.46 \\
Textiles & 2625 & -0.65 \\
Paper, printing, publishing & 64 & -0.09 \\
Other manufacturing & 3444 & -0.59 \\
\hline
\end{tabular}

Table 14. - Comparison of occupancy specific correlations

\begin{tabular}{|c|c|c|c|c|c|c|c|}
\hline \multirow[b]{2}{*}{ Building size $\left(\mathrm{m}^{2}\right)$} & \multicolumn{6}{|c|}{$\begin{array}{l}\text { Ratio of occupancy specific fire frequency to all- } \\
\text { manufacturing prediction }\end{array}$} & \multirow{2}{*}{$\begin{array}{l}\text { Fire } \\
\text { potential }\end{array}$} \\
\hline & 10 & $10^{2}$ & $10^{3}$ & $10^{4}$ & $10^{5}$ & $10^{6}$ & \\
\hline All manufacturing & 1.00 & 1.00 & 1.00 & $1.0 \overline{0}$ & 1.00 & 1.00 & nominal \\
\hline Food, drink, tobacco & 0.76 & 0.89 & 1.05 & 1.23 & 1.45 & 1.70 & minal \\
\hline Chemical and allied & 3.45 & 2.94 & 2.50 & 2.13 & 1.81 & 1.54 & high \\
\hline gineering & 0.10 & 0.16 & 0.27 & 0.45 & 0.74 & 1.23 & low \\
\hline Electrical engineering & 0.41 & 0.47 & 0.53 & 0.61 & 0.70 & 0.81 & low \\
\hline Vehicle manufacture & 0.13 & 0.27 & 0.57 & 1.23 & 2.63 & 5.62 & nominal \\
\hline Metal goods & 0.96 & 0.99 & 1.01 & 1.03 & 1.06 & 1.08 & nominal \\
\hline rext & 2.91 & 1.93 & 1.2 & 0.84 & 0.56 & 0.37 & nominal \\
\hline publishing & 0.10 & 0.24 & 0.57 & 1.36 & 3.27 & 7.85 & $\ldots$ \\
\hline Other manufacturing & 3.75 & 2.84 & 2.16 & 1.64 & 1.24 & 0.94 & $\ldots$ \\
\hline
\end{tabular}

Table 14 provides the ratio of the estimated fire frequency (fires/yr) and the estimated fire frequency for all industry over a range of areas. From this information it is possible to estimate an appropriate multiplier for each occupancy. To simplify implementation the occupancies were grouped into similar types. Since the basis for the correlations is not available, some of the occupancy descriptions are rater obsicure. This grouping process allows the occupancies to be defined explicitly. The logical groups are low, nominal and high potential. These groups were defined based on the frequency estimates at $1000 \mathrm{~m}^{2}$ : 
$\begin{array}{cc}\text { low } & \frac{F}{F_{\text {all }}} \leq 0.7 \\ \text { (18) nominal } & 0.7<\frac{F}{F_{\text {all }}} \leq 1.3 \\ \text { high } & 1.3<\frac{F}{F_{\text {all }}}\end{array}$

Table 15 summarizes the results for the fire potential grouping. The data for vehicle manufacture and paper, printing, publishing have been excluded because of their slopes are very shallow. The other manufacturing category is also excluded from the analysis since this category is ill defined.

Table 15.-Industrial fire frequency ratios

\begin{tabular}{l|c|c|c}
\hline Fire potential & $\begin{array}{c}\text { Number in } \\
\text { category }\end{array}$ & Mean & $\begin{array}{c}\text { Standard } \\
\text { deviation }\end{array}$ \\
\hline low & 2 & 0.4 & 0.2 \\
nominal & 4 & 1.0 & 0.3 \\
high & 1 & 2.5 & $\ldots$ \\
\hline
\end{tabular}

This analysis approach is repeated in Table 16 for the SRS Production Occupancy results. Ranking the ratios in order demonstrates that below a value of 1.0 most of the large buildings are related to reactor type operations. Above a value of 1.5 the buildings are related to material processing activities or laboratories. These ranges will be denoted as low, nominal and high.

The respective mean values of the ratios for each group are presented in Table 17. These mean values do not represent the best estimator for the $\mathrm{K}$ multiplier. In some cases the data is sparse. In some cases some of the building use has be inconsistent. (e.g., Building 105- $\mathrm{L}$ was in a non-operational state and essentially abandoned for more than 10 years.) It may also be skewed because there are several buildings with a particular occupancy. The recommended $\mathrm{K}$ multipliers presented in Table 17 are based on the information in Table 16 and the author's judgement. 
Table 16. - SRS production facility derivation of occupancy multiplier

\begin{tabular}{|c|c|c|c|c|c|c|c|}
\hline \multirow[b]{2}{*}{ Building } & \multicolumn{3}{|c|}{ Historical data } & \multicolumn{2}{|c|}{$\begin{array}{c}\text { All-manufacturing } \\
\text { correlation }\end{array}$} & \multicolumn{2}{|c|}{$\begin{array}{c}\text { SRS } \\
\text { Correlation }\end{array}$} \\
\hline & $\begin{array}{c}F^{\prime \prime} \\
\text { fires/km } \mathrm{km}^{2} \cdot \mathrm{yr}\end{array}$ & $\begin{array}{c}\text { Area } \\
\mathrm{m}^{2}\end{array}$ & $\begin{array}{c}F \\
\text { fires/yr }\end{array}$ & $\begin{array}{c}F \\
\text { fires/yr }\end{array}$ & $\mathrm{K}$ & $\begin{array}{c}F \\
\text { fires/yr }\end{array}$ & $\mathbf{K}$ \\
\hline $105-000 \mathrm{~L}$ & 1.7 & 46452 & 0.08 & 0.51 & 0.16 & 0.72 & 0.11 \\
\hline $105-000 \mathrm{R}$ & 4.2 & 46452 & 0.20 & 0.51 & 0.39 & 0.72 & 0.27 \\
\hline $184-000 \mathrm{~L}$ & 10.3 & 7897 & 0.08 & 0.20 & 0.41 & 0.29 & 0.28 \\
\hline 184-000R & 10.3 & 7897 & 0.08 & 0.20 & 0.41 & 0.29 & 0.28 \\
\hline $105-000 \mathrm{P}$ & 5.8 & 69677 & 0.41 & 0.63 & 0.65 & 0.89 & 0.46 \\
\hline 484-000D & 11.6 & 23226 & 0.27 & 0.35 & 0.77 & 0.50 & 0.54 \\
\hline $105-000 \mathrm{~K}$ & 8.7 & 46452 & 0.41 & 0.51 & 0.80 & 0.72 & 0.56 \\
\hline $105-000 \mathrm{C}$ & 10.5 & 46452 & 0.49 & 0.51 & 0.96 & 0.72 & 0.67 \\
\hline $234-000 \mathrm{H}$ & 31.3 & 5331 & 0.17 & 0.16 & 1.04 & 0.23 & 0.71 \\
\hline $210-000 S$ & 28.7 & 8696 & 0.25 & 0.21 & 1.20 & 0.30 & 0.83 \\
\hline $232-000 \mathrm{H}$ & 45.0 & 5130 & 0.23 & 0.16 & 1.47 & 0.23 & 1.01 \\
\hline $184-000 \mathrm{~K}$ & 37.6 & 7897 & 0.30 & 0.20 & 1.50 & 0.29 & 1.04 \\
\hline 184-000P & 41.1 & 7897 & 0.32 & 0.20 & 1.64 & 0.29 & 1.13 \\
\hline 221-000S & 28.1 & 17782 & 0.50 & 0.30 & 1.64 & 0.44 & 1.14 \\
\hline $221-000 \mathrm{H}$ & 26.9 & 30621 & 0.82 & 0.41 & 2.03 & 0.58 & 1.42 \\
\hline $247-000 F$ & 48.1 & 13006 & 0.63 & 0.26 & 2.43 & 0.37 & 1.68 \\
\hline $321-000 \mathrm{M}$ & 88.2 & 5209 & 0.46 & 0.16 & 2.90 & 0.23 & 1.99 \\
\hline $772-000 F$ & 77.1 & 7785 & 0.60 & 0.20 & 3.06 & 0.28 & 2.11 \\
\hline $320-000 \mathrm{M}$ & 91.9 & 5880 & 0.54 & 0.17 & 3.20 & 0.25 & 2.19 \\
\hline $313-000 \mathrm{M}$ & 143.3 & 5315 & 0.76 & 0.16 & 4.75 & 0.23 & 3.26 \\
\hline $221-000 \mathrm{~F}$ & 76.7 & 30632 & 2.35 & 0.41 & 5.79 & 0.58 & 4.04 \\
\hline 773-000A & 82.8 & 28267 & 2.34 & 0.39 & 6.02 & 0.56 & 4.20 \\
\hline
\end{tabular}

Table 17. - SRS fire frequency ratios

\begin{tabular}{l|c|c|c}
\hline Fire potential & $\begin{array}{c}\text { All-manufacturing } \\
\text { correlation }\end{array}$ & $\begin{array}{c}\text { SRS } \\
\text { correlation }\end{array}$ & $\begin{array}{c}\text { Recommended } \\
\text { K multiplier }\end{array}$ \\
\hline Low & 0.6 & 0.4 & 0.8 \\
Nominal & 1.2 & 0.8 & 1.2 \\
High & 3.2 & 2.2 & 3.0 \\
\hline
\end{tabular}




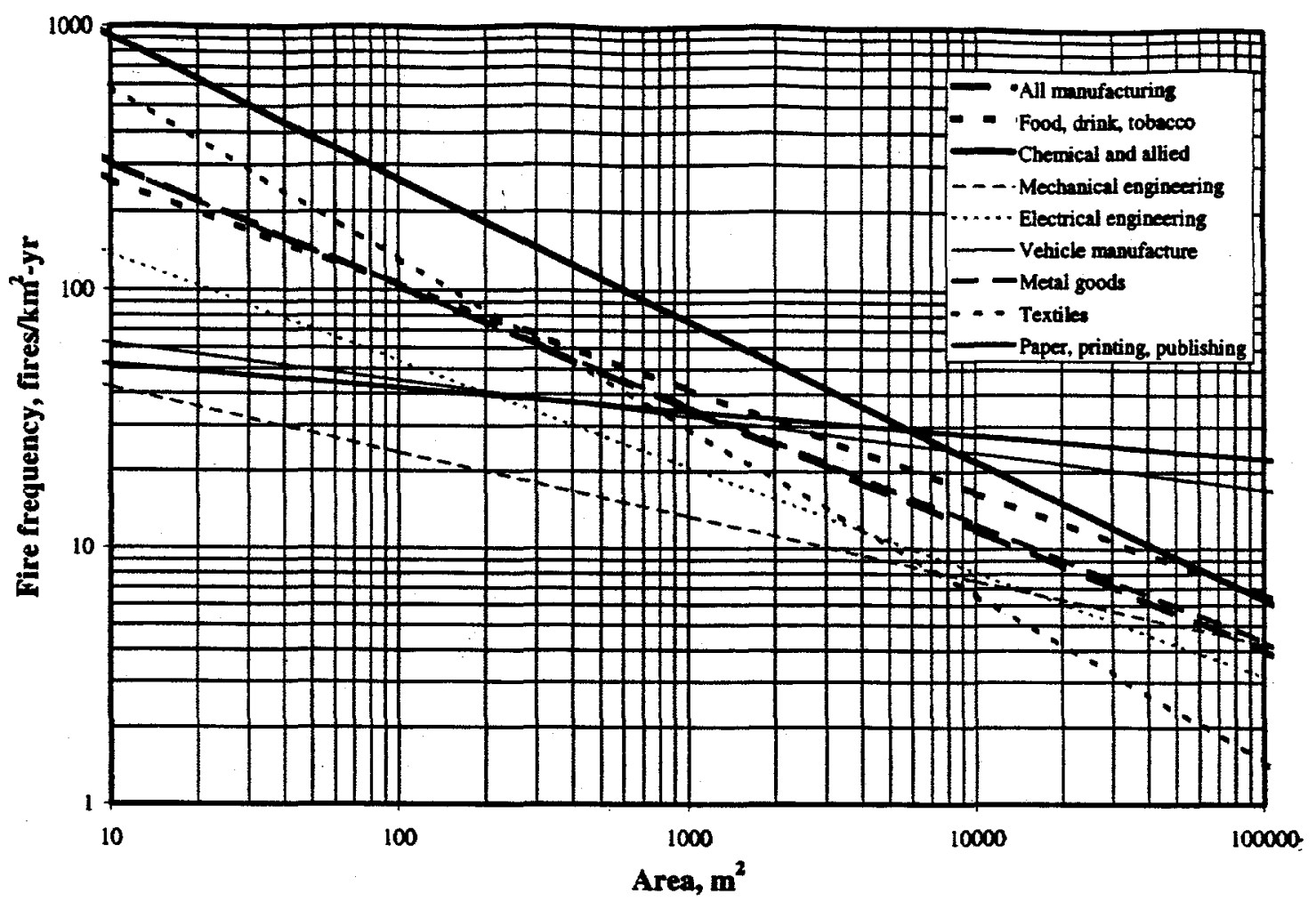

Figure 6, Fire frequency predictions for industrial facilities

\section{Implementation}

The incipient fire frequencies presented in this report are intended to estimate the frequency of an incipient fire occurring in a specific building. As such only one correlation should be applied to any one building. In a mixed occupancy (e.g., offices and industrial activities in a single building) the correlation that best represents the overall occupancy should be selected and a single incipient fire frequency can then be computed. Multiple correlations should not be used to estimate the frequencies in a single building unless the building segmented by construction that create independent occupancies (i.e., 4-hour blank fire rated construction.)

There is nothing in the above approach that mandates that the incipient fire frequency be distributed uniformly over the building. If known the incipient frequency can be distributed based on room use. Such information is readily available in single and multifamily residences. In industrial occupancies the weighting can be derived from the occupancy multipliers in Table 17. 
WSRC-TR-99-00361

\section{Works Cited}

1. Coutts, D. A. 1994. Fire Risk Assessment Methodology Generic Event Tree Description (U). Aiken, SC: Westinghouse Savaunah River Company. (March) WSRC-TR-94-0188.

2. Fire Safety Engineering in Buildings, Part 1: Guide to the Application of Fire Safety Engineering Principles. 1997. London, England: British Standards Institute. DD240: Part 1: 1997.

3. Fire Engineering Guidelines. 1996. Sydney, New South Wales, Australia: Fire Code Reform Centre Limited.

4. Ford, H. A., D. F. Paddleford, and M. J. Vitacco. 1992. Benefit vs Cost Evaluation Methodology for Fire Protection Upgrade Projects (U). Aiken, SC: Westinghouse Savannah River Company. (May) VrSRC-TR-92-141.

5. Brandyberry, M. D. 1990. Analysis of SRS Industrial Fire Records (U). Aiken, SC: Westinghouse Savannah River Company. (Jariuary) WSRC-TR-90-143.

6. Westinghouse Savannah River Site Fire Department 1991 Annual Report. 1992. Aiken, SC: Westinghouse Savannah River Compariy.

7. Westinghouse Savannah River Site Fire Department 1992 Annual Report. 1992. Aiken, SC: Westinghouse Savannah River Company.

8. Westinghouse Savannah River Site Fire Department 1993 Annual Report. 1994. Aiken, SC: Westinghouse Savannah River Company.

9. Westinghouse Savannah River Site Fire Department 1994 Annual Report. 1995. Aiken, SC: Westinghouse Savannah River Company. 
WSRC-TR-99-00361

Appendix A, SRS Fire Loss Data 
This page intentionally blank. 
Table A-1.--Incipient fire data for A Area

\begin{tabular}{|c|c|c|c|c|c|c|c|c|c|}
\hline \multirow[b]{2}{*}{ Area } & \multirow[b]{2}{*}{ Type } & \multirow[b]{2}{*}{ ID } & \multirow[b]{2}{*}{ Area, $\mathbf{f t}^{2}$} & \multirow[b]{2}{*}{ Area, $\mathbf{m}^{2}$} & \multirow{2}{*}{$\begin{array}{c}\text { years in } \\
\text { service to } \\
94\end{array}$} & \multirow[b]{2}{*}{ yrs $^{*} m^{2}$} & \multicolumn{3}{|c|}{ Fire count } \\
\hline & & & & & & & $\begin{array}{c}\text { Original } \\
\text { count }\end{array}$ & '91 to '94| & $\begin{array}{l}\text { Sum of } \\
\text { all fires }\end{array}$ \\
\hline & & $9 y$ & MOG & 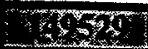 & & 76009 & 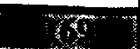 & $\pi$ & W \\
\hline$A$ & $\mathrm{~A}$ & $305-000 \mathrm{~A}$ & 18606 & 1729 & 37 & 63973 & 0 & 0 & 0 \\
\hline $\mathbf{A}$ & $\mathbf{A}$ & $607-002 \mathrm{~A}$ & 240 & 22 & 41 & 902 & 2 & 0 & 2 \\
\hline $\bar{A}$ & $\bar{A}$ & 607-016A & 600 & 56 & 7 & 392 & 0 & 0 & $\overline{0}$ \\
\hline$\overline{\mathbf{A}}$ & A & 614-001A & 86 & $\overline{8}$ & 41 & 328 & 0 & 0 & $\overline{0}$ \\
\hline $\bar{A}$ & A & $701-001 \mathrm{~A}$ & 873 & $\overline{81}$ & 40 & 3240 & 0 & 0 & $\overline{0}$ \\
\hline A & $\mathrm{A}$ & $701-002 \mathrm{~A}$ & 170 & 16 & 41 & 656 & $\overline{0}$ & 0 & 0 \\
\hline $\bar{A}$ & $\bar{A}$ & $701-003 A$ & 150 & 14 & 41 & 574 & 0 & 0 & $\frac{0}{0}$ \\
\hline A & $\bar{A}$ & $701-009 A$ & 64 & $\overline{6}$ & 9 & 54 & 0 & 0 & 0 \\
\hline A & $\mathrm{A}$ & $701-012 \mathrm{~A}$ & 1216 & 113 & 6 & 678 & $\overline{0}$ & 0 & 0 \\
\hline $\bar{A}$ & $\mathrm{~A}$ & $701-013 A$ & 48 & $\overline{4}$ & 8 & 32 & $\overline{0}$ & 0 & $\overline{0}$ \\
\hline $\bar{A}$ & $\bar{A}$ & $702-000 \mathrm{~A}$ & 5195 & 483 & 37 & 17871 & 2 & 0 & 2 \\
\hline A & A & 703-000A & 177000 & 16444 & 37 & 608428 & 7 & $\overline{3}$ & 10 \\
\hline A & $\bar{A}$ & $703-002 A$ & 1536 & 143 & 14 & 2002 & $\overline{0}$ & 0 & $\overline{0}$ \\
\hline $\bar{A}$ & $\bar{A}$ & $703-003 \mathrm{~A}$ & 1536 & 143 & 9 & 1287 & 0 & 0 & $\overline{0}$ \\
\hline $\mathbf{A}$ & A & $1703-004 A$ & 1560 & 145 & 14 & 2030 & 0 & 0 & $\overline{0}$ \\
\hline A & $\mathrm{A}$ & $703-005 \mathrm{~A}$ & 1560 & 145 & 9 & 1305 & $\overline{0}$ & 0 & $\overline{0}$ \\
\hline A & $\mathrm{A}$ & $703-006 \mathrm{~A}$ & 1536 & 143 & 9 & 1287 & $\overline{0}$ & $\overline{0}$ & $\overline{0}$ \\
\hline $\mathbf{A}$ & $A$ & $703-009 \mathrm{~A}$ & 823 & 76 & 9 & 684 & $\overline{0}$ & 0 & $\overline{0}$ \\
\hline $\mathbf{A}$ & $\mathrm{A}$ & $703-010 \mathrm{~A}$ & 1536 & 143 & 9 & 1287 & $\overline{0}$ & 0 & $\overline{0}$ \\
\hline $\mathbf{A}$ & $\bar{A}$ & $703-011 \mathrm{~A}$ & 1536 & 143 & 9 & 1287 & $\overline{0}$ & 0 & $\overline{0}$ \\
\hline $\bar{A}$ & $\bar{A}$ & $703-012 \mathrm{~A}$ & 720 & 67 & 9 & 603 & $\overline{0}$ & 0 & $\overline{0}$ \\
\hline $\bar{A}$ & $\sqrt{\mathrm{A}}$ & $703-013 A$ & 720 & 67 & 9 & 603 & $\overline{0}$ & 0 & 0 \\
\hline $\mathrm{A}$ & $\sqrt{A}$ & $703-014 \mathrm{~A}$ & 1536 & 143 & 9 & 1287 & $\overline{0}$ & 0 & $\overline{0}$ \\
\hline $\bar{A}$ & A & $703-015 A$ & 720 & 67 & 9 & 603 & $\overline{0}$ & 0 & $\overline{0}$ \\
\hline $\bar{A}$ & $\bar{A}$ & $703-016 \mathrm{~A}$ & 720 & 67 & 9 & 603 & $\overline{0}$ & 0 & $\overline{0}$ \\
\hline $\bar{A}$ & $\sqrt{\mathrm{A}}$ & $703-017 \mathrm{~A}$ & 720 & 67 & 9 & 603 & $\overline{0}$ & 0 & 0 \\
\hline$\overline{\mathrm{A}}$ & $\widehat{A}$ & $703-018 A$ & 720 & 67 & 9 & 603 & $\overline{0}$ & 0 & $\overline{0}$ \\
\hline A & $\mathrm{A}$ & $703-022 \mathrm{~A}$ & 1792 & 166 & 9 & 1494 & 0 & 0 & 0 \\
\hline A & $A$ & $703-026 A$ & 1792 & 166 & 9 & 1494 & 0 & 0 & 0 \\
\hline A & $A$ & $703-028 \mathrm{~A}$ & 1792 & 166 & 9 & 1494 & 0 & 0 & 0 \\
\hline A & A & $703-030 \mathrm{~A}$ & 1792 & 166 & 14 & 2324 & 0 & 0 & 0 \\
\hline $\mathbf{A}$ & $\mathrm{A}$ & $703-031 \mathrm{~A}$ & 1792 & 166 & 14 & 2324 & $\overline{0}$ & 0 & 0 \\
\hline A & $\mathrm{A}$ & $703-033 A$ & 1536 & 143 & 14 & 2002 & $\overline{0}$ & $\overline{0}$ & 0 \\
\hline $\mathrm{A}$ & $\bar{A}$ & $703-034 \mathrm{~A}$ & 1600 & 149 & 14 & 2086 & $\overline{0}$ & $\overline{0}$ & 0 \\
\hline $\bar{A}$ & $\bar{A}$ & $703-037 A$ & 1600 & 149 & 14 & 2086 & $\overline{0}$ & $\overline{0}$ & $\overline{0}$ \\
\hline $\bar{A}$ & $\bar{A}$ & $703-038 \mathrm{~A}$ & 1600 & 149 & 14 & 2086 & 0 & 0 & $\overline{0}$ \\
\hline $\mathbf{A}$ & $A$ & 703-041A & 20000 & 1858 & 14 & 26012 & $\overline{0}$ & 1 & 1 \\
\hline $\mathbf{A}$ & $\mathbf{A}$ & 703-042A & 20000 & 1858 & 14 & 26012 & 0 & 1 & 1 \\
\hline $\mathbf{A}$ & $\bar{A}$ & $703-043 A$ & 20000 & 1858 & 14 & 26012 & 4 & 0 & $\overline{4}$ \\
\hline A & $\mathbf{A}$ & 703-044A & 20000 & 1858 & 5 & 9290 & 1 & 0 & 1 \\
\hline A & $\mathrm{A}$ & $703-045 \mathrm{~A}$ & 20000 & 1858 & 5 & 9290 & 0 & 0 & 0 \\
\hline A & $\mathrm{A}$ & $703-051 \mathrm{~A}$ & 432 & 40 & 7 & 280 & $\overline{0}$ & $\overline{0}$ & 0 \\
\hline $\bar{A}$ & $\sqrt{A}$ & $703-052 A$ & 896 & $\overline{83}$ & 7 & 581 & $\overline{0}$ & $\overline{0}$ & 0 \\
\hline $\bar{A}$ & $\widehat{A}$ & $703-053 A$ & 896 & $\overline{83}$ & 7 & 581 & 0 & $\overline{0}$ & $\overline{0}$ \\
\hline A & $\bar{A}$ & $703-054 \mathrm{~A}$ & 896 & 83 & 7 & 581 & $\overline{0}$ & $\overline{0}$ & 0 \\
\hline $\mathbf{A}$ & $\bar{A}$ & $703-055 \mathrm{~A}$ & 896 & $\overline{83}$ & 7 & 581 & $\overline{0}$ & 0 & 0 \\
\hline
\end{tabular}


Table A-1.--Incipient fire data for A Area

\begin{tabular}{|c|c|c|c|c|c|c|c|c|c|}
\hline \multirow[b]{2}{*}{ Area } & \multirow[b]{2}{*}{ Type } & \multirow[b]{2}{*}{ ID } & \multirow[b]{2}{*}{ Area, $\mathbf{f}^{2}$} & \multirow[b]{2}{*}{ Area, $\mathbf{m}^{2}$} & \multirow{2}{*}{\begin{tabular}{|c|} 
years in \\
service to \\
94
\end{tabular}} & \multirow[b]{2}{*}{$\mathrm{yrs}^{*} \mathbf{m}_{1}^{2}$} & \multicolumn{3}{|c|}{ Fire count } \\
\hline & & & & & & & $\begin{array}{c}\text { Original } \\
\text { count }\end{array}$ & |'91 to '94| & $\begin{array}{l}\text { Sum of } \\
\text { all fires }\end{array}$ \\
\hline A & $A$ & 703-056A & 896 & 83 & 7 & 581 & 0) & 0 & 0 \\
\hline$A$ & $\bar{A}$ & $703-057 \mathrm{~A}$ & 896 & 83 & 7 & 581 & 0 & 0 & 0 \\
\hline$A$ & $\mathrm{~A}$ & $703-058 \mathrm{~A}$ & 896 & 83 & 7 & 581 & 0 & 0 & 0 \\
\hline$A$ & $\mathrm{~A}$ & $703-063 \mathrm{~A}$ & 896 & 83 & 9 & 747 & 0 & 0 & 0 \\
\hline $\bar{A}$ & $\overline{\mathrm{A}}$ & $703-070 \mathrm{~A}$ & 896 & 83 & 9 & 747 & 0 & 0 & 0 \\
\hline$A$ & $\mathbf{A}$ & $703-071 \mathrm{~A}$ & 600 & 56 & 9 & 504 & 0 & 0 & 0 \\
\hline$A$ & $\bar{A}$ & $703-073 \mathrm{~A}$ & 1792 & 166 & 6 & 996 & of & 0 & 0 \\
\hline $\mathrm{A}$ & $\mathbf{A}$ & $706-000 \mathrm{~A}$ & 1216 & 113 & 41 & 4633 & 0 & 0) & 0 \\
\hline $\bar{A}$ & $\overline{\mathrm{A}}$ & $707-000 \mathrm{~A}$ & 1008 & 94 & 41 & 3854 & 0 & 0 & 0 \\
\hline$\widehat{A}$ & $\bar{A}$ & 708-000A & 40068 & 3722 & 37 & 137714 & 7 & 0 & 7 \\
\hline $\mathrm{A}$ & $\overline{\mathbf{A}}$ & 709-000A & 3695 & 343 & 41 & $14 \overline{063}$ & 0 & 0 & 0 \\
\hline A & $\mathbf{A}$ & $710-000 \mathrm{~A}$ & 9533 & 886 & 41 & 36326 & 2 & 01 & 2 \\
\hline $\mathbf{A}$ & A & $711-000 \mathrm{~A}$ & 2512 & 233 & \begin{tabular}{l|l}
41 \\
\end{tabular} & 9553 & 0 & 0 & 0 \\
\hline A & A & $712-000 \mathrm{~A}$ & 683 & 631 & 41 & 2583 & 0 & 이 & 0 \\
\hline A & A & $713-000 \mathrm{~A}$ & 48521 & 4508 & 41 & 184.828 & 0 & 0 & 0 \\
\hline $\mathrm{A}$ & A & $713-001 \mathrm{~A}$ & 11760 & 1093 & 43 & 46999 & 1 & 0 & 1 \\
\hline $\bar{A}$ & $\bar{A}$ & $713-002 \mathrm{~A}$ & 20000 & 1858 & 11 & $2(438$ & 0 & of & 0 \\
\hline A & $\mathrm{A}$ & $713-003 \mathrm{~A}$ & 896 & 83 & 6 & 498 & 0 & 0 & 0 \\
\hline$A$ & $\bar{A}$ & $714-000 \mathrm{~A}$ & 45036 & 4184 & 41 & 171.544 & 0 & 0 & 0 \\
\hline A & $\bar{A}$ & $715-000 \mathrm{~A}$ & 229 & 21 & 41 & 861 & 1 & 1 & 2 \\
\hline A & A & $716-000 \mathrm{~A}$ & 100 & 9 & 41 & 369 & 7 & 1 & 8 \\
\hline A & A & $716-001 \mathrm{~A}$ & 896 & 83 & 5 & 415 & 0 & 0 & 0 \\
\hline$A$ & $\mathrm{~A}$ & $716-002 \mathrm{~A}$ & 7225 & 671 & 5) & 3355 & 2 & 1 & 3 \\
\hline A & A & $716-003 A$ & 360 & 33 & 8 & 264 & 0 & 0 & 0 \\
\hline$A$ & $A$ & $716-004 \mathrm{~A}$ & 2400 & 223 & 8 & 1784 & 0 & 0 & 0 \\
\hline$A$ & $\mathrm{~A}$ & $717-000 \mathrm{~A}$ & 52435 & 4871 & 41 & 199711 & 9 & 0 & 9 \\
\hline $\mathrm{A}$ & $\mathrm{A}$ & $717-004 \mathrm{~A}$ & 200 & 19 & 10 & 190 & 0 & 0 & 0 \\
\hline $\mathrm{A}$ & $\mathrm{A}$ & $717-005 \mathrm{~A}$ & 1536 & 143 & 15 & 2145 & 0 & 0 & 0 \\
\hline A & $A$ & $717-006 \mathrm{~A}$ & 160 & 15 & 9 & 135 & 0 & 0 & 0 \\
\hline $\mathrm{A}$ & $A$ & $717-007 \mathrm{~A}$ & 1536 & 143 & 10 & 1430 & 0 & 0 & 0 \\
\hline$A$ & A & $717-008 \mathrm{~A}$ & 792 & 74 & 7 & 518 & 0 & 0 & 0 \\
\hline $\bar{A}$ & $\widehat{A}$ & $717-009 \mathrm{~A}$ & 792 & 74| & 7 & 518 & 0 & 0 & 0 \\
\hline $\mathrm{A}$ & $\mathbf{A}$ & 717-010A & 1440 & 134 & 8 & 1072 & 0 & of & 0 \\
\hline A & A & $719-000 \mathrm{~A}$ & 26487 & 2461 & 37 & 91057 & 0 & 0 & 0 \\
\hline $\mathrm{A}$ & A & 719-001A & 840 & 78 & 6 & 468 & 0 & 0 & 0 \\
\hline$\overline{\mathbf{A}}$ & $\bar{A}$ & $719-002 \mathrm{~A}$ & 1792 & 166 & 6 & 996 & of & 0 & 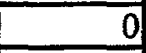 \\
\hline A & $A$ & $719-003 \mathrm{~A}$ & 160 & 15 & 13 & 195 & 0 & 0 & $\overline{0}$ \\
\hline$A$ & $A$ & 719-004A & 16000 & 1486 & 9 & 13374 & 0 & 0 & 0 \\
\hline A & $A$ & $719.007 \mathrm{~A}$ & 896 & 83 & 7 & 581 & 0 & 0 & 0 \\
\hline $\mathrm{A}$ & A & $719-008 \mathrm{~A}$ & 896 & 83 & 6 & 498 & 1 & 0 & 1 \\
\hline $\bar{A}$ & $\bar{A}$ & $719-009 \mathrm{~A}$ & 896 & 83 & 6 & 498 & 0 & 0 & 0 \\
\hline $\mathrm{A}$ & $A$ & $719-011 \mathrm{~A}$ & 900 & 84 & 6 & 504 & 0 & 1 & 1 \\
\hline$A$ & $\bar{A}$ & $719-013 A$ & 504 & 47 & 5 & 235 & 0 & 0 & 0 \\
\hline $\mathbf{A}$ & A & $719-014 A$ & 952 & 88 & 5| & 440 & 0 & 0 & 0 \\
\hline $\bar{A}$ & $\bar{A}$ & $719-015 A$ & 952 & 88 & 5 & 440 & 0 & 0 & 0 \\
\hline A & $A$ & $719-016 \mathrm{~A}$ & 952 & 88 & 5 & 440 & 0 & 0 & 0 \\
\hline A & $\widehat{A}$ & $719-017 \mathrm{~A}$ & 962 & 89 & 5 & 445 & 0 & 0 & 0 \\
\hline
\end{tabular}


Table A-1.--Incipient fire data for A Area

\begin{tabular}{|c|c|c|c|c|c|c|c|c|c|}
\hline \multirow[b]{2}{*}{ Area } & \multirow[b]{2}{*}{ Type } & \multirow[b]{2}{*}{ ID } & \multirow[b]{2}{*}{ Area, $\mathbf{f t}^{2}$} & \multirow[b]{2}{*}{ Area, $\mathbf{m}^{2}$} & \multirow{2}{*}{$\begin{array}{c}\text { years in } \\
\text { service to } \\
194\end{array}$} & \multirow[b]{2}{*}{ yrs* $\mathbf{m}^{2}$} & \multicolumn{3}{|c|}{ Fire count } \\
\hline & & & & & & & $\begin{array}{c}\text { Original } \\
\text { count }\end{array}$ & & $\begin{array}{l}\text { Sum of } \\
\text { all fires }\end{array}$ \\
\hline A & A & $719-018 \mathrm{~A}$ & 1792 & 166 & 5) & 830 & 이 & 이 & 0 \\
\hline $\mathbf{A}$ & $\mathrm{A}$ & $719-019 \mathrm{~A}$ & 1792 & 166 & 5 & 830 & of & 0 & 0 \\
\hline $\mathbf{A}$ & $\mathrm{A}$ & $719-020 \mathrm{~A}$ & 720 & 67 & 5 & 335 & of & 0 & 0 \\
\hline A & A & $720-000 \mathrm{~A}$ & 11180 & 1039 & 41 & 42599 & 0 & 0 & $\overline{0}$ \\
\hline A & $\mathrm{A}$ & $721.000 \mathrm{~A}$ & 4595 & 427 & 17 & 7259 & 0 & 0 & 0 \\
\hline A & A & $722-000 \mathrm{~A}$ & 11700 & 1087 & 37) & 40219 & 0 & 0 & 0 \\
\hline $\mathbf{A}$ & A & $722-001 \mathrm{~A}$ & 6900 & 641 & 13 & 8333 & 0 & 0 & $\overrightarrow{0}$ \\
\hline A & $\mathrm{A}$ & $722-002 \mathrm{~A}$ & 720 & 67 & 14 & 938 & 0 & 0 & 0 \\
\hline A & A & $722-004 \mathrm{~A}$ & 7800 & 725 & 11 & 7975 & 0 & 0 & 0 \\
\hline $\bar{A}$ & $\mathbf{A}$ & $722-005 A$ & 20000 & 1858 & 5 & 9290 & 0 & 0 & 0 \\
\hline A & A & $722.008 \mathrm{~A}$ & 792 & 74 & 6 & 444 & 0 & 0 & $\overline{0}$ \\
\hline A & $\mathrm{A}$ & $723-000 \mathrm{~A}$ & 25194 & 2341 & 38 & 88958 & 31 & 1) & 4 \\
\hline A & $\mathrm{A}$ & 723-002A & 1152 & 107 & 18 & 1926 & 0 & 이 & 0 \\
\hline A & A & $723-004 \mathrm{~A}$ & 1792 & 166 & 17| & 2822 & 0 & 0) & 0 \\
\hline A & A & $723-005 \mathrm{~A}$ & 384 & 36 & 17 & 612 & 0 & 0 & 0 \\
\hline A & A & $723-006 \mathrm{~A}$ & 1320 & 123 & 17 & 2091 & 0 & 0 & 0 \\
\hline A & A & $723-007 A$ & 1560 & 145 & 17 & 2465 & 0 & 0 & 0 \\
\hline $\mathbf{A}$ & A & $723-008 \mathrm{~A}$ & 720 & 67 & 16 & 1072 & 0 & 0 & 0 \\
\hline $\mathrm{A}$ & A & $723-009 \mathrm{~A}$ & 720 & 67 & 16 & 1072 & 0 & 0 & 0 \\
\hline $\mathbf{A}$ & $A$ & $723-012 \mathrm{~A}$ & 240 & 22 & 13 & 286 & 0 & 0 & 0 \\
\hline $\bar{A}$ & A & $723-013 \mathrm{~A}$ & 210 & 20 & 14 & 280 & 0 & 0 & 0 \\
\hline $\bar{A}$ & A & $723-015 A$ & 2400 & 223 & 6 & 1338 & 0 & 0 & 0 \\
\hline $\mathrm{A}$ & A & $724-000 \mathrm{~A}$ & 11595 & 1077 & 41 & 44157 & 0 & 0 & 0 \\
\hline A & A & 724-002A & 2160 & 201 & 17 & 3417 & 이 & 0 & 0 \\
\hline A & A & 724-003A & 1536 & 1.43 & 7 & 1001 & 0 & 의 & 0 \\
\hline A & $A$ & 724-004A & 1536 & 143 & 7 & 1001 & 0] & 0] & 0 \\
\hline A & A & $724-006 \mathrm{~A}$ & 384 & 36 & 8 & 288 & 의 & 의 & 0 \\
\hline A & A & 724-007A & 384 & 36 & 8 & 288 & 0 & 의 & 0 \\
\hline A & A & $725-000 \mathrm{~A}$ & 1680 & 156 & 39 & 6084 & 0 & 요 & 0 \\
\hline A & A & $726-000 \mathrm{~A}$ & 400 & 37 & 39 & 1443 & 0] & 이 & 0 \\
\hline A & A & $730-000 \mathrm{~A}$ & 20000 & 1858 & 9 & 16722 & 1 & 0 & 1 \\
\hline A & A & $730-001 \mathrm{~A}$ & 1792 & 166 & 6 & 996 & of & 0 & 0 \\
\hline A & A & $730-002 \mathrm{~A}$ & 1792 & 166 & 6 & 996 & 0 & 0 & 0 \\
\hline $\bar{A}$ & $\mathbf{A}$ & $730-003 \mathrm{~A}$ & 1792 & 166 & 6 & 996 & 이 & 0 & 0 \\
\hline $\bar{A}$ & $\mathbf{A}$ & $733-000 \mathrm{~A}$ & 1926 & 179 & 41 & 7339 & 0 & 0 & 0 \\
\hline A & $\mathbf{A}$ & $733-001 \mathrm{~A}$ & 4000 & 372 & 6 & 2232 & 01 & 0 & 0 \\
\hline A & $\mathbf{A}$ & 734-000A & 2034 & 189 & 41 & 7749 & 0 & 0 & 0 \\
\hline $\mathrm{A}$ & $\mathbf{A}$ & $735-000 \mathrm{~A}$ & 48290 & 4486 & 41 & 183926 & 13 & 0 & 13 \\
\hline A & $\mathbf{A}$ & $735-002 \mathrm{~A}$ & 1800 & 167 & 20 & 3340 & of & 1 & 7 \\
\hline $\mathbf{A}$ & A & $735-004 A$ & 213 & 20 & 18 & 360 & of & 0 & 0 \\
\hline A & $\mathbf{A}$ & 735-007A & 7500 & 697 & 11 & 7667 & 의 & 0 & 0 \\
\hline $\bar{A}$ & $\mathbf{A}$ & $735-008 \mathrm{~A}$ & 1536 & 143 & 14 & 2002 & 0 & 0 & 0 \\
\hline A & $\mathbf{A}$ & $735-009 \mathrm{~A}$ & 1536 & 143 & 12 & 1716 & 0 & 0 & 0 \\
\hline $\mathrm{A}$ & A & $735-011 \mathrm{~A}$ & 30000 & 2787 & 6 & 16722 & 0 & 0 & $\overline{0}$ \\
\hline A & A & $735-013 \mathrm{~A}$ & 1800 & 167 & 6 & 1002 & 0 & 0 & $\underline{0}$ \\
\hline A & A & $735-016 \mathrm{~A}$ & 1792 & 166 & 6 & 996 & 0 & 0 & 0 \\
\hline A & $\mathbf{A}$ & $736-000 \mathrm{~A}$ & 10286 & 956 & 37 & 35372 & 0 & 0 & $\underline{0}$ \\
\hline
\end{tabular}


Table A-1.--Incipient fire data for A Area

\begin{tabular}{|c|c|c|c|c|c|c|c|c|c|}
\hline \multirow[b]{2}{*}{ Area } & \multirow[b]{2}{*}{ Type } & \multirow[b]{2}{*}{ ID } & \multirow[b]{2}{*}{ Area, $\mathbf{f t}^{2}$} & \multirow[b]{2}{*}{ Area, $\mathbf{m}^{2}$} & \multirow{2}{*}{\begin{tabular}{|c|} 
years in \\
service to \\
'94
\end{tabular}} & \multirow[b]{2}{*}{$\mathrm{yrs}^{*} \mathrm{mi}^{2}$} & \multicolumn{3}{|c|}{ Fire count } \\
\hline & & & & & & & $\begin{array}{c}\text { Origina } \\
\text { count }\end{array}$ & '91 to $94 \mid$ & $\begin{array}{l}\text { Sum of } \\
\text { all fires }\end{array}$ \\
\hline A & A & $736-001 \mathrm{~A}$ & 120 & 11 & 37 & 407 & 이 & 0) & 0 \\
\hline $\mathrm{A}$ & A & $737-000 \mathrm{~A}$ & 27321 & 2538 & 17 & 43146 & of & 0 & 0 \\
\hline A & A & $737-001 \mathrm{~A}$ & 2702 & 251 & 12 & 3012 & 01 & 0 & 0 \\
\hline $\mathrm{A}$ & A & $737-002 \mathrm{~A}$ & 1210 & 112 & 10 & 1120 & 0 & 0 & 0 \\
\hline $\bar{A}$ & $A$ & $737-003 \mathrm{~A}$ & 1356 & 126 & 10 & 1260 & o) & 이 & 0 \\
\hline$\widehat{A}$ & A & $737-004 \mathrm{~A}$ & 740 & 69 & 10 & 690 & 0 & 0 & 0 \\
\hline $\bar{A}$ & A & $737-005 \mathrm{~A}$ & 2400 & 223 & 20 & $\triangle 460$ & 0 & 0 & 0 \\
\hline $\mathrm{A}$ & A & $737-006 \mathrm{~A}$ & 1584 & 147 & 9 & 1323 & of & 0 & 0 \\
\hline $\bar{A}$ & A & $737-007 \mathrm{~A}$ & 1440 & 134 & 9 & 206 & 0 & 0 & 0 \\
\hline$A$ & A & $737-008 \mathrm{~A}$ & 1440 & 134 & 9 & 206 & 0 & 0 & 0 \\
\hline$A$ & $\mathrm{~A}$ & $737-011 \mathrm{~A}$ & 1475 & 137 & 9 & 1233 & of & 0 & 0 \\
\hline $\bar{A}$ & $\mathrm{~A}$ & $737-012 A$ & 738 & 69 & 9 & 621 & of & 0 & 0 \\
\hline $\mathbf{A}$ & $\bar{A}$ & $737-013 \mathrm{~A}$ & 5000 & 465 & 8 & 3720 & of & 0 & 0 \\
\hline $\mathbf{A}$ & $\mathrm{A}$ & $737-014 A$ & 1440 & 134 & 9 & 1206 & of & 0 & 0 \\
\hline A & A & $737-015 \mathrm{~A}$ & 1440 & 134 & 9] & 1206 & 0 & 0 & 0 \\
\hline A & A & $737-017 \mathrm{~A}$ & 500 & 46 & 9 & 414 & 0 & 0 & 0 \\
\hline $\mathbf{A}$ & $A$ & $737-018 \mathrm{~A}$ & 2400 & 223 & 8 & 1784 & 0 & 0 & 0 \\
\hline$A$ & 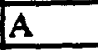 & $737-019 \mathrm{~A}$ & 1440 & 134 & 7 & 938 & 0 & 0 & 0 \\
\hline $\mathbf{A}$ & $\mathbf{A}$ & $737-020 \mathrm{~A}$ & 768 & 71 & 9 & 639 & 0 & 0 & 0 \\
\hline $\mathrm{A}$ & $\mathrm{A}$ & $737-023 \mathrm{~A}$ & 300 & 28 & 9 & 252 & 0 & 0 & 0 \\
\hline $\mathbf{A}$ & $\mathrm{A}$ & $738-000 \mathrm{~A}$ & 96 & 9 & 41 & 369 & 0 & 0 & 0 \\
\hline $\bar{A}$ & $\mathrm{~A}$ & $740-000 \mathrm{~A}$ & 3026 & 281 & 41 & 11521 & 0 & 0 & 0 \\
\hline $\mathbf{A}$ & $\mathrm{A}$ & $740-003 \mathrm{~A}$ & 1792 & 166 & 6 & 996 & 0 & 0 & 0 \\
\hline$A$ & A & $740-004 \mathrm{~A}$ & 1792 & 166 & 6 & 996 & 0 & 0 & 0 \\
\hline $\mathrm{A}$ & $\widehat{A}$ & $740-005 \mathrm{~A}$ & 1792 & 166 & 6 & 996 & 0 & 0 & 0 \\
\hline A & A & $740-006 \mathrm{~A}$ & 1792 & 166 & 6 & 996 & 0 & 0 & 0 \\
\hline $\mathbf{A}$ & $\mathrm{A}$ & $740-007 \mathrm{~A}$ & 504 & 47 & 5 & 235 & 0 & 0 & 0 \\
\hline$A$ & $A$ & $740-008 \mathrm{~A}$ & 1200 & 111 & 5) & 555 & 0 & 0 & 0 \\
\hline $\bar{A}$ & A & $740-009 \mathrm{~A}$ & 1792 & 166 & 5 & 830 & 0 & 0 & 0 \\
\hline $\bar{A}$ & A & $740-010 \mathrm{~A}$ & 1792 & 166 & 5 & 830 & 0 & 0 & 0 \\
\hline $\mathbf{A}$ & $\mathbf{A}$ & $740-011 \mathrm{~A}$ & 1792 & 166 & 5 & 830 & 0 & 0 & 0 \\
\hline $\mathrm{A}$ & $\mathrm{A}$ & $740-012 \mathrm{~A}$ & 1792 & 166 & 5 & 830 & 0 & 0 & 0 \\
\hline $\bar{A}$ & $\bar{A}$ & $742-000 \mathrm{~A}$ & 29000 & 2694 & 11 & 29634 & 0 & 0 & 0 \\
\hline$A$ & $A$ & $742-002 \mathrm{~A}$ & 1792 & 166 & 6 & 996 & 0 & 0 & 0 \\
\hline $\mathrm{A}$ & $\bar{A}$ & $742-003 \mathrm{~A}$ & 1792 & 166 & 6 & 996 & 0 & 0 & 0 \\
\hline A & $\mathbf{A}$ & $742-004 \mathrm{~A}$ & 720 & 67 & 6 & 402 & 0 & 0 & 0 \\
\hline A & A & $742-005 \mathrm{~A}$ & 720 & 67 & 6 & 402 & 0 & 0 & 0 \\
\hline $\mathbf{A}$ & $\widehat{A}$ & $743-000 \mathrm{~A}$ & 1034 & 96 & 41 & 3936 & 0 & 0 & 0 \\
\hline A & A & $743-001 \mathrm{~A}$ & 1440 & 134 & 14. & 1876 & 0 & 0 & 0 \\
\hline $\mathrm{A}$ & $\mathrm{A}$ & $745-000 \mathrm{~A}$ & 11025 & 1024 & 36 & 36864 & 1 & 0 & 1 \\
\hline $\bar{A}$ & $\bar{A}$ & $745-003 \mathrm{~A}$ & 384 & 36 & 11 & 396 & 0 & 0 & 0 \\
\hline A & $\bar{A}$ & $745-006 \mathrm{~A}$ & 2400 & 223 & 11 & 2453 & 0 & 0 & 0 \\
\hline $\mathrm{A}$ & $\mathrm{A}$ & $746-000 \mathrm{~A}$ & 1296 & 120 & 36 & 4320 & 0 & 0 & 0 \\
\hline$A$ & A & $746-001 \mathrm{~A}$ & 1200 & 111 & 14 & 1554 & 0 & 0 & 0 \\
\hline $\bar{A}$ & A & $748-000 \mathrm{~A}$ & 5680 & 528 & 27 & 14256 & 0 & 0 & 0 \\
\hline $\mathbf{A}$ & $\bar{A}$ & $748-001 \mathrm{~A}$ & 35 & 3 & 27 & 81 & 0 & 0 & 0 \\
\hline$A$ & $A$ & $749-000 \mathrm{~A}$ & 11500 & 1068 & 6 & 6408 & 0 & 0 & 0 \\
\hline
\end{tabular}


Table A-1.--Incipient fire data for A Area

\begin{tabular}{|c|c|c|c|c|c|c|c|c|c|}
\hline \multirow[b]{2}{*}{ Area } & \multirow[b]{2}{*}{ Type } & \multirow[b]{2}{*}{ ID } & \multirow[b]{2}{*}{ Area, $\mathbf{f t}^{2}$} & \multirow[b]{2}{*}{ Area, $\mathbf{m}^{2}$} & \multirow{2}{*}{\begin{tabular}{|c|} 
years in \\
service to \\
.94
\end{tabular}} & \multirow[b]{2}{*}{$y r{ }^{*} m^{2}$} & \multicolumn{3}{|c|}{ Fire count } \\
\hline & & & & & & & $\begin{array}{c}\text { Original } \\
\text { count }\end{array}$ & '91 to '94] & $\begin{array}{l}\text { Sum of } \\
\text { all fires }\end{array}$ \\
\hline$A$ & A & 749-001A & 100 & 9 & 6 & 54 & 0 & 0 & 0 \\
\hline A & A & $751-001 \mathrm{~A}$ & 4450 & 413 & 37. & 15281 & 1 & 0 & 1 \\
\hline A & A & $751-002 \mathrm{~A}$ & 288 & 27 & 41 & 1107 & 0 & 0 & 0 \\
\hline $\mathrm{A}$ & A & $752-025 \mathrm{~A}$ & 200 & 19 & 19 & 361 & 0 & 0 & 0 \\
\hline$A$ & A & $761-000 \mathrm{~A}$ & 141 & 13 & 37 & 481 & 0 & 0 & 0 \\
\hline$A$ & $A$ & $763-000 \mathrm{~A}$ & 2400 & 223 & 15 & 3345 & 0 & 0 & 0 \\
\hline A & A & $770-000 \mathrm{~A}$ & 1404 & 130 & 41 & 5330 & 0 & 0 & 0 \\
\hline $\bar{A}$ & A & $771-000 \mathrm{~A}$ & 45 & 4 & 39 & 156 & 0 & $\overline{0}$ & $\overline{0}$ \\
\hline A & A & $773-000 \mathrm{~A}$ & 304263 & 28267 & 41 & 1158947 & 96 & 0 & 96 \\
\hline A & A & $773-002 \mathrm{~A}$ & 1012 & 94 & 41 & 3854 & 0 & 0 & 0 \\
\hline A & A & $773-003 \mathrm{~A}$ & 449 & 42 & 41 & 1722 & 0 & 0 & 0 \\
\hline$A$ & A & $773-004 \mathrm{~A}$ & 35 & 3 & 28 & 84 & 0 & 0 & 0 \\
\hline $\mathbf{A}$ & $\mathbf{A}$ & $773-005 \mathrm{~A}$ & 35 & 3 & 28 & 84 & 0 & 0 & $\overline{0}$ \\
\hline A & A & $773-006 A$ & 35 & 3 & 26 & 78 & 0 & 0 & 0 \\
\hline $\mathbf{A}$ & A & $773-008 A$ & 35 & 3 & 25 & 75 & 0 & 0 & $\overline{0}$ \\
\hline $\mathbf{A}$ & $\bar{A}$ & $773-011 \mathrm{~A}$ & 1824 & 169 & 9 & 1521 & I) & 0 & 1 \\
\hline $\mathbf{A}$ & $\mathrm{A}$ & $773-012 \mathrm{~A}$ & 1632 & 152 & 9 & 1368 & 0 & 0 & 0 \\
\hline A & A & $773-013 A$ & 1500 & 139 & 9 & 1251 & 0 & 이 & 0 \\
\hline A & A & 773-014A & 768 & 71 & 9 & 639 & 0] & 0 & 0 \\
\hline A & A & 773-016A & 1536 & 143 & 9 & 1287 & 0 & 0 & 0 \\
\hline $\mathbf{A}$ & A & $773-017 \mathrm{~A}$ & 1536 & 143 & 9 & 1287 & 0 & 0 & 0 \\
\hline A & A & 773-018A & 1536 & 143 & 9 & 1287 & 0| & 0 & 0 \\
\hline A & $\mathbf{A}$ & $773-019 A$ & 1536 & 143 & 9 & 1287 & of & 이 & 0 \\
\hline A & A & $773-020 \mathrm{~A}$ & 896 & 83 & 16 & 1328 & 0 & 0 & 0 \\
\hline $\mathrm{A}$ & A & $773-021 \mathrm{~A}$ & 1512 & 140 & 16 & 2240 & of & 0 & 0 \\
\hline A & A & $773-022 \mathrm{~A}$ & 1536 & 143 & 14 & 2002 & 0 & 0 & 0 \\
\hline $\mathrm{A}$ & A & $773-023 A$ & 1536 & 143 & 14 & 2002 & 0 & 0 & 0 \\
\hline A & $\bar{A}$ & $773-024 \mathrm{~A}$ & 1536 & 143 & 14 & 2002 & 0 & 0 & $\overline{0}$ \\
\hline $\mathbf{A}$ & $\mathrm{A}$ & $773-026 \mathrm{~A}$ & 1536 & 143 & 9 & 1287 & of & 0 & 0 \\
\hline $\mathrm{A}$ & A & $773-027 A$ & 1536 & 143 & $\overline{9}$ & 1287 & 0 & 0 & $\overline{0}$ \\
\hline $\mathbf{A}$ & A & $773-041 \mathrm{~A}$ & 20000 & 1858 & 10 & 18580 & 0 & 0 & 0 \\
\hline $\bar{A}$ & A & $773-042 \mathrm{~A}$ & 20000 & 1858 & 9 & 16722 & 0 & 0 & $\overline{0}$ \\
\hline$\overline{\mathbf{A}}$ & $\bar{A}$ & $773-043 A$ & 18720 & 1739 & 7 & 12173 & 0 & 0 & $\overline{0}$ \\
\hline $\bar{A}$ & A & $773-051 \mathrm{~A}$ & 20000 & 1858 & $\overline{6}$ & 11148 & 0 & 0 & $\overline{0}$ \\
\hline A & A & $773-053 \mathrm{~A}$ & 1792 & 166 & 6 & 996 & 0 & 0 & $\underline{0}$ \\
\hline $\bar{A}$ & A & $773-054 A$ & 1792 & 166 & $\overline{6}$ & 996 & 0 & 0 & $\overline{0}$ \\
\hline $\mathbf{A}$ & $\mathbf{A}$ & $773-055 \mathrm{~A}$ & 1792 & 166 & 6 & 996 & 0 & 0 & 0 \\
\hline $\mathbf{A}$ & $\bar{A}$ & $773-056 \mathrm{~A}$ & $\overline{1792}$ & 166 & 5 & 830 & 0 & 0 & 0 \\
\hline $\bar{A}$ & $\mathbf{A}$ & $773-057 A$ & 1792 & 166 & 5 & 830 & 0 & 0 & 0 \\
\hline $\bar{A}$ & A & $773-058 \mathrm{~A}$ & 1792 & 166 & 5 & 830 & 0 & 0 & 0 \\
\hline A & A & $773-059 A$ & 1792 & 166 & 5 & 830 & 0 & 0 & 0 \\
\hline$A$ & A & $773-060 \mathrm{~A}$ & 504 & 47 & 9 & 423 & 0 & 0 & $\overline{0}$ \\
\hline $\bar{A}$ & A & $774-000 \mathrm{~A}$ & 2501 & 232 & 37 & 8584 & 1 & 0 & 1 \\
\hline A & A & 774-001A & 720 & 67 & 8 & 536 & 0 & 이 & 0 \\
\hline A & A & $774-002 \mathrm{~A}$ & 798 & 74 & 8 & 592 & 0 & 0 & 0 \\
\hline $\mathrm{A}$ & A & $775-000 \mathrm{~A}$ & 819 & 76 & 25 & 1900 & 0 & 0 & $\overline{0}$ \\
\hline $\bar{A}$ & A & $776-000 \mathrm{~A}$ & 3000 & 279 & 6 & 1674 & 0 & 0 & 0 \\
\hline
\end{tabular}


Table A-1.--Incipient fire data for A Area

\begin{tabular}{|c|c|c|c|c|c|c|c|c|c|}
\hline \multirow[b]{2}{*}{ Area } & \multirow[b]{2}{*}{ Type } & \multirow[b]{2}{*}{ I } & \multirow[b]{2}{*}{ Area, $\mathrm{n}^{2}$} & \multirow[b]{2}{*}{ Area, $\mathrm{m}^{2}$} & \multirow{2}{*}{$\begin{array}{c}\text { years in } \\
\text { service to } \\
94\end{array}$} & \multirow[b]{2}{*}{$y\left(s^{*} m^{2}\right.$} & \multicolumn{3}{|c|}{ Fire count } \\
\hline & & & & & & & $\begin{array}{c}\text { Origina } \\
\text { count }\end{array}$ & $\mid 91$ to $94 \mid$ & $\begin{array}{l}\text { Sum of } \\
\text { all fires }\end{array}$ \\
\hline$A$ & $A$ & $776-001 \mathrm{~A}$ & 1801 & 167 & 41 & 6847 & 0 & 0 & 0 \\
\hline $\mathrm{A}$ & $A$ & $776-003 \mathrm{~A}$ & 280 & 26 & 26 & 676 & $\overline{0}$ & 0 & 0 \\
\hline $\mathrm{A}$ & A & $776-004 \mathrm{~A}$ & 280 & 26 & 26 & 676 & 0 & 0 & 0 \\
\hline $\mathrm{A}$ & A & $776-005 \mathrm{~A}$ & 360 & 33 & 8 & 264 & 0 & 0 & 0 \\
\hline A & A & $776-006 \mathrm{~A}$ & 2563 & 238 & 41 & 9758 & $\overline{0}$ & 0 & 0 \\
\hline A & A & $776-008 \mathrm{~A}$ & 608 & 56 & 9 & 504 & 0 & 0 & 0 \\
\hline A & A & $777-000 \mathrm{~A}$ & 2800 & 260 & 5 & 1300 & 0 & 0 & 0 \\
\hline $\mathrm{A}$ & A & $777.010 \mathrm{~A}$ & 55308 & 5138 & 41 & 21.0658 & 1 & 2 & 3 \\
\hline A & $\mathrm{A}$ & $777-011 \mathrm{~A}$ & 96 & 9 & 41 & 369 & 0 & 0 & 0 \\
\hline $\mathrm{A}$ & $\mathrm{A}$ & $777-012 \mathrm{~A}$ & 885 & 82 & 30 & 2460 & $\overline{0}$ & 0 & 0 \\
\hline A & $\mathbf{A}$ & $777-015 \mathrm{~A}$ & 720 & 67 & 7 & 469 & $\overline{0}$ & o & 0 \\
\hline $\mathbf{A}$ & $\mathrm{A}$ & $778-000 \mathrm{~A}$ & 144 & 13 & 33 & 429 & 0 & 0 & 0 \\
\hline $\mathbf{A}$ & $\mathrm{A}$ & $778.005 \mathrm{~A}$ & & 0 & & & 0 & 1 & 1 \\
\hline $\mathrm{A}$ & $\mathrm{A}$ & $779.000 \mathrm{~A}$ & 4589 & 426 & 24 & 0224 & 1 & 0 & 1 \\
\hline A & $\mathrm{A}$ & $779-001 \mathrm{~A}$ & 720 & 67 & 9 & 603 & 0 & 0 & 0 \\
\hline A & A & $779.003 \mathrm{~A}$ & 150 & 14 & 9 & 126 & 0 & 0) & 0 \\
\hline A & A & $779-006 \mathrm{~A}$ & 288 & 27 & 9 & 243 & $\overline{0}$ & 0 & 0 \\
\hline$A$ & A & $779-010 \mathrm{~A}$ & 1450 & 135 & 9 & 1215 & 0 & 0 & 0 \\
\hline $\mathbf{A}$ & A & $780-001 \mathrm{~A}$ & 270 & 25 & 41 & 1025 & $\overline{0}$ & 0 & 0 \\
\hline A & A & $780-002 \mathrm{~A}$ & 421 & 39 & 41 & 1599 & $\overline{0}$ & 0 & 0 \\
\hline A & A & $781-000 \mathrm{~A}$ & 10800 & 1003 & 9 & 9027 & 0 & 0 & 0 \\
\hline A & A & $784-000 \mathrm{~A}$ & 6000 & 557 & 37 & 20609 & 2 & 1 & $\overline{3}$ \\
\hline A & A & $784001 \mathrm{~A}$ & 960 & 89 & 13 & 1157 & 0 & 0 & 0 \\
\hline A & A & $784-003 \mathrm{~A}$ & 150 & 14 & 14 & 196 & 0 & 이 & 0 \\
\hline A & $A$ & $784-004 \mathrm{~A}$ & 480 & 45 & 14 & 630 & 0 & 0 & 0 \\
\hline A & A & $784-004 \mathrm{~A}$ & 173 & 16 & 14 & 224 & 0 & 0 & 0 \\
\hline A & A & $785-000 \mathrm{~A}$ & 960 & 89 & 41 & 3649 & 0 & 0 & 0 \\
\hline A & A & $785-001 \mathrm{~A}$ & 121 & 11 & 30 & 330 & 0 & 0 & 0 \\
\hline A & A & $786-000 \mathrm{~A}$ & 4600 & 427 & 22 & 9394 & 0 & 0 & 0 \\
\hline A & $\mathrm{A}$ & $786-001 \mathrm{~A}$ & 1536 & 143 & 14 & 2002 & 0 & 0 & 0 \\
\hline $\mathbf{A}$ & $\mathrm{A}$ & $786-002 \mathrm{~A}$ & 408 & 38 & 14 & 532 & 0 & 0 & 0 \\
\hline $\mathbf{A}$ & $\mathrm{A}$ & $786-004 \mathrm{~A}$ & 288 & 27 & 8 & 216 & 0 & 0 & 0 \\
\hline A & $\mathrm{A}$ & $786-005 \mathrm{~A}$ & 2100 & 195 & 9 & 1755 & 0 & 0 & 0 \\
\hline $\mathbf{A}$ & A & $786-006 \mathrm{~A}$ & 1120 & 104 & 9) & 936 & $\overline{0}$ & 0 & 0 \\
\hline $\mathbf{A}$ & $\bar{A}$ & $786-013 A$ & 144 & 13 & 9 & 117 & $\overline{0}$ & 0 & 0 \\
\hline $\bar{A}$ & $\mathbf{A}$ & $789-000 \mathrm{~A}$ & 6000 & 557 & 37 & 20609 & 2 & 2 & 4 \\
\hline A & $A$ & $792-000 \mathrm{~A}$ & 882 & 82 & 20 & 1640 & $\overline{0}$ & 0 & 0 \\
\hline A & $\mathbf{A}$ & $905.098 \mathrm{~A}$ & 196 & 18 & 13 & 234 & $\overline{0}$ & 0 & 0 \\
\hline
\end{tabular}


Table A-2.--Incipient fire data for B Area

\begin{tabular}{|c|c|c|c|c|c|c|c|c|c|}
\hline \multirow[b]{2}{*}{ Area } & \multirow[b]{2}{*}{ Type } & \multirow[b]{2}{*}{ ID } & \multirow[b]{2}{*}{ Area, $\mathrm{f}^{2}$} & \multirow[b]{2}{*}{ Area, $m^{2}$} & \multirow{2}{*}{$\begin{array}{c}\text { years in } \\
\text { service to } \\
.94\end{array}$} & \multirow[b]{2}{*}{$\mathrm{yrs}^{*} \mathrm{~m}^{2}$} & \multicolumn{3}{|c|}{ Fire count } \\
\hline & & & & & & & $\begin{array}{c}\text { Original } \\
\text { count }\end{array}$ & '91 to 94 & $\begin{array}{l}\text { Sum of } \\
\text { all fires }\end{array}$ \\
\hline & & $\sqrt{10}$ & $\mathrm{rat}$ & 36 & & 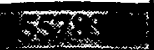 & 8 & 4 & 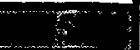 \\
\hline B & A & $607-002 \mathrm{~B}$ & 432 & 40 & 7 & 280 & 0 & 0 & 0 \\
\hline $\bar{B}$ & $\sqrt{A}$ & $623-000 \mathrm{~B}$ & o & $\overline{0}$ & 0 & $\overrightarrow{0}$ & 1 & $\overline{0}$ & 1 \\
\hline$\overline{\mathbf{B}}$ & A & $703-000 \mathrm{~B}$ & 20000 & 1858 & 8 & 14864 & 1 & 1 & 2 \\
\hline $\bar{B}$ & $\bar{A}$ & $703-001 B$ & 20000 & 1858 & 8 & 14864 & 1 & $\overline{1}$ & 2 \\
\hline $\bar{B}$ & A & $703-005 B$ & 20000 & 1858 & 7 & 13006 & $\overline{0}$ & $\overline{0}$ & 0 \\
\hline $\mathbf{B}$ & A & $703-007 \mathrm{~B}$ & 952 & $\overline{88}$ & 6 & 528 & $\overline{0}$ & $\overline{0}$ & 0 \\
\hline $\bar{B}$ & $A$ & $703-010 \mathrm{~B}$ & 480 & $\overline{45}$ & 6 & 270 & $\overline{0}$ & $\overline{0}$ & 0 \\
\hline $\bar{B}$ & $\bar{A}$ & $703-012 B$ & 1200 & 111 & 6 & 666 & $\overline{0}$ & $\overline{0}$ & 0 \\
\hline B & A & $704-000 \mathrm{~B}$ & 3000 & 279 & 9 & 2511 & $\overline{0}$ & $\overline{0}$ & $\overline{0}$ \\
\hline B & A & 704-003B & 1800 & 167 & 8 & 1336 & 0 & 0 & 0 \\
\hline B & $A$ & $707-032 \mathrm{~B}$ & 504 & 47 & 9 & 423 & 0 & 0 & 0 \\
\hline $\bar{B}$ & $\mathbf{A}$ & $707-033 B$ & 504 & 47 & 9 & 423 & $\overline{0}$ & $\overline{0}$ & 0 \\
\hline $\bar{B}$ & $\mathbf{A}$ & $716-000 \mathrm{~B}$ & 4624 & 430 & 9 & 3870 & 0 & $\overline{0}$ & 0 \\
\hline$\overline{\mathbf{B}}$ & A & $772-026 B$ & 1920 & 178 & 6 & 1068 & $\overline{0}$ & 0 & 0 \\
\hline $\bar{B}$ & A & $772-027 \mathrm{~B}$ & 3000 & 279 & 6 & 1674 & $\overline{0}$ & $\overline{0}$ & 0 \\
\hline
\end{tabular}


Table A-3.--Incipient fire data for 700C Area

\begin{tabular}{|c|c|c|c|c|c|c|c|c|c|}
\hline & & & & & years in & & & Fire coun & \\
\hline Area & Type & I & Area, $\mathrm{ft}^{2}$ & Area, $\mathbf{m}^{2}$ & $\begin{array}{c}\text { service to } \\
94\end{array}$ & $y_{r s}{ }^{*} m^{2}$ & $\begin{array}{c}\text { Original } \\
\text { count }\end{array}$ & ' 91 to '94 & $\begin{array}{l}\text { Sum of } \\
\text { all fires }\end{array}$ \\
\hline & & ST. & 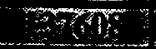 & 78 & & 79 & $=$ & & \\
\hline C & A & $701-001 \mathrm{C}$ & 4164 & 387 & Y1 & 3483 & 0 & 0 & 0 \\
\hline $\bar{C}$ & A & $701-002 \mathrm{C}$ & 1679 & 156 & 9 & 1404 & $\underline{0}$ & $\overline{0}$ & $\pi$ \\
\hline $\bar{C}$ & $\mathrm{~A}$ & $701-004 C$ & 240 & 22 & 9 & 198 & 0 & $\overline{0}$ & $\overline{0}$ \\
\hline C & $A$ & $701-005 \mathrm{C}$ & 240 & 22 & 9 & 198 & $\underline{0}$ & $\overline{0}$ & 0 \\
\hline $\bar{C}$ & A & $701-006 \mathrm{C}$ & 200 & 19 & 9 & 171 & $\overline{0}$ & $\overline{0}$ & 0 \\
\hline $\bar{c}$ & $\mathbf{A}$ & $702-000 \mathrm{C}$ & 1665 & 155 & 9 & 1395 & $\underline{0}$ & $\overline{0}$ & $\underline{0}$ \\
\hline $\bar{C}$ & A & $704-000 \mathrm{C}$ & 18489 & 1718 & 41 & 70438 & 4 & $\overline{0}$ & 4 \\
\hline $\bar{c}$ & $\mathrm{~A}$ & $704-001 \mathrm{C}$ & \begin{tabular}{|l|}
1493 \\
\end{tabular} & 139 & 91 & 1251 & $\underline{0}$ & $\overline{0}$ & 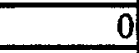 \\
\hline $\bar{c}$ & $A$ & $704-002 \mathrm{C}$ & 1792 & 166 & 9 & 1494 & $\overline{0}$ & $\overline{0}$ & $\underline{0}$ \\
\hline $\bar{c}$ & $A$ & $704-006 \mathrm{C}$ & 4826 & 448 & 6 & 2688 & $\underline{0}$ & $\overline{1}$ & \\
\hline c & A & $705-000 \mathrm{C}$ & 26250 & 2439 & 9 & 21951 & $\overline{0}$ & 0 & 0 \\
\hline$\overline{\mathbf{C}}$ & $\bar{A}$ & $706-000 \mathrm{C}$ & 19000 & 1765 & 39 & 68835 & $\underline{0}$ & $\overline{1}$ & \\
\hline $\bar{c}$ & A & $706-006 \mathrm{C}$ & 720 & 67 & 9 & 603 & $\overline{0}$ & $\overline{0}$ & 0 \\
\hline $\bar{C}$ & A & $706-009 C$ & 3920 & 364 & 9] & 3276 & 0 & 1 & \\
\hline $\bar{c}$ & A & $706-017 \mathrm{C}$ & 1792 & 166 & 9| & 1494 & 0 & 0 & $\underline{0}$ \\
\hline $\bar{c}$ & $\mathrm{~A}$ & $707-000 \mathrm{C}$ & 44728 & 4155 & $7 \pi$ & 29085 & $\overline{0}$ & $\overline{0}$ & \\
\hline $\bar{C}$ & $\mathbf{A}$ & $707-002 \mathrm{C}$ & 540 & 50 & 2 & 100 & 0 & 0 & 0 \\
\hline$\overline{\mathrm{C}}$ & $\bar{A}$ & $707-003 \mathrm{C}$ & 540 & 50 & 2 & 100 & $\overline{0}$ & 0 & $\underline{0}$ \\
\hline $\bar{c}$ & $\mathbf{A}$ & $707-004 C$ & 540 & 50 & 2 & 100 & $\underline{0}$ & O & $\underline{0}$ \\
\hline $\bar{C}$ & $\mathrm{~A}$ & $707-005 C$ & 540 & 50 & 4 & 100 & $\underline{0}$ & - & $\underline{0}$ \\
\hline C & $\mathrm{A}$ & $717-000 \mathrm{C}$ & 4250 & 395 & 9 & 3555 & & 0 & \\
\hline & & & & & & & & & \\
\hline
\end{tabular}


Table A-4.--Incipient fire data for 700D Area

\begin{tabular}{|c|c|c|c|c|c|c|c|c|c|}
\hline \multirow[b]{2}{*}{ Area } & \multirow[b]{2}{*}{ Type } & \multirow[b]{2}{*}{$\mathbf{D}$} & \multirow[b]{2}{*}{ Area, $\mathrm{ft}^{2}$} & \multirow[b]{2}{*}{ Area, $\mathbf{m}^{2}$} & \multirow{2}{*}{$\begin{array}{c}\text { years in } \\
\text { service to } \\
.94\end{array}$} & \multirow[b]{2}{*}{ grs* $m^{2}$} & \multicolumn{3}{|c|}{ Fire count } \\
\hline & & & & & & & $\begin{array}{c}\text { Original } \\
\text { count }\end{array}$ & '91 to '94| & $\begin{array}{l}\text { Sum of } \\
\text { all fires }\end{array}$ \\
\hline & & $\sqrt{2}$ & ory & Trs & & HET & & 8 & 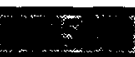 \\
\hline D & $A$ & 701-001D & 4034 & 375 & 41 & 15375 & 0 & 0 & 0 \\
\hline D & A & $701-002 \mathrm{D}$ & 100 & 9 & 41 & 369 & $\overline{0}$ & 0 & 0 \\
\hline D & A & 701-003D & 202 & 19 & 41 & 779 & 0 & 0 & 0 \\
\hline D & A & 704-000D & 10087 & 937 & 41 & 38417 & 1 & 0 & 1 \\
\hline $\bar{D}$ & A & 704-001D & 1792 & 166 & $\overline{6}$ & 996 & $\overline{0}$ & 0 & $\overline{0}$ \\
\hline D & A & 707-000D & 1665 & 155 & 41 & 6355 & 1 & 0 & 1 \\
\hline D & A & 711-000D & 680 & 63 & 41 & 2583 & 0 & 0 & 0 \\
\hline D & A & 711-001D & 3646 & 339 & 7 & 2373 & 0 & 0 & 0 \\
\hline D & $A$ & $717-000 \mathrm{D}$ & 18365 & 1706 & 41 & 69946 & 1 & 0 & 1 \\
\hline $\bar{D}$ & A & $772-000 \mathrm{D}$ & 11750 & 1092 & 41 & 44772 & 2 & 0 & 2 \\
\hline
\end{tabular}


Table A-5.--Incipient fire data for 700F Area

\begin{tabular}{|c|c|c|c|c|c|c|c|c|c|}
\hline \multirow[b]{2}{*}{ Area } & \multirow[b]{2}{*}{ Type } & \multirow[b]{2}{*}{ ID } & \multirow[b]{2}{*}{ Area, $\mathrm{ft}^{2}$} & \multirow[b]{2}{*}{ Area, $\mathrm{m}^{2}$} & \multirow{2}{*}{$\begin{array}{c}\text { years in } \\
\text { service to } \\
94\end{array}$} & \multirow[b]{2}{*}{$\operatorname{yrs}^{*} \mathbf{m}^{2}$} & \multicolumn{3}{|c|}{ Fire count } \\
\hline & & & & & & & $\begin{array}{c}\text { Original } \\
\text { count }\end{array}$ & [91 to 94 & $\begin{array}{l}\text { Sum of } \\
\text { all fires }\end{array}$ \\
\hline & & ST: & $7 \mathrm{TSE}$ & 3ing & & 780 & 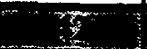 & & Ts \\
\hline$F$ & A & $300-000 \mathrm{~F}$ & 0 & 0 & o & 0 & 1 & 0 & 1 \\
\hline $\mathbf{F}$ & A & $701-001 \mathrm{~F}$ & 6080 & 565 & 40 & 22600 & 0 & 1 & 1 \\
\hline$F$ & A & $701-002 \mathrm{~F}$ & 150 & 14 & 40 & 560 & 0 & 0 & 0 \\
\hline $\mathbf{F}$ & A & $701-003 \mathrm{~F}$ & 672 & 62 & 40 & 2480 & 0 & o & 0 \\
\hline$F$ & A & $701-004 \mathrm{~F}$ & 150 & 14 & 40 & 560 & 0 & of & 0 \\
\hline$F$ & A & $701-005 \mathrm{~F}$ & 228 & 21 & 40 & 840 & 0 & 0 & 0 \\
\hline$F$ & A & $701-006 \mathrm{~F}$ & 308 & 29 & 9 & 261 & 0 & 0 & 0 \\
\hline $\mathbf{F}$ & A & $701-007 \mathrm{~F}$ & 144 & 13 & 9 & 117 & 0 & 0 & $\overline{0}$ \\
\hline F & A & $701-009 \mathrm{~F}$ & 1800 & 167 & 6 & 1002 & 0 & 0 & 0 \\
\hline $\mathbf{F}$ & $\mathbf{A}$ & $701-013 \mathrm{~F}$ & 308 & 29 & 6 & 174 & 0 & & 0 \\
\hline$F$ & A & $701-018 \mathrm{~F}$ & 308 & 29 & 5 & 145 & 0 & & 0 \\
\hline$F$ & A & $701-019 \mathrm{~F}$ & 1440 & 134 & 5ा & 670 & 0 & 5 & $\overline{0}$ \\
\hline $\mathbf{F}$ & A & $702-000 \mathrm{~F}$ & 1665 & 155 & 4 & 620 & 0 & 0 & 0 \\
\hline$F$ & A & $703-000 \mathrm{~F}$ & 25000 & 2323 & 10 & $2: 1230$ & 1 & 0 & 1 \\
\hline$F$ & A & $703-001 \mathrm{~F}$ & 840 & 78 & 10 & 780 & 0 & 0 & 0 \\
\hline$F$ & A & $704-000 \mathrm{~F}$ & 12141 & 1128 & 40 & 45120 & 2 & 0 & 2 \\
\hline$F$ & A & $704-002 \mathrm{~F}$ & 1296 & 120 & 9 & 080 & 0 & 0 & $\overline{0}$ \\
\hline$F$ & A & $704-003 F$ & 1296 & 120 & 19 & 2280 & 0 & 0 & $\overline{0}$ \\
\hline$F$ & $\mathbf{A}$ & $704-004 \mathrm{~F}$ & 720 & 67 & 9 & 603 & 0 & 0 & 0 \\
\hline $\mathbf{F}$ & A & $704-005 \mathrm{~F}$ & 720 & 67 & 14 & 938 & 0 & 0 & $\overline{0}$ \\
\hline$F$ & A & $704-008 \mathrm{~F}$ & 1536 & 143 & 12 & 1716 & D & 0) & 0 \\
\hline$F$ & A & $704-010 \mathrm{~F}$ & 1792 & 166 & 12 & 1992 & 0 & 0 & 0 \\
\hline $\mathbf{F}$ & A & $704-011 \mathrm{~F}$ & 896 & 83 & 11 & 913 & 0 & 0 & 0 \\
\hline $\mathbf{F}$ & A & $704-012 \mathrm{~F}$ & 896 & 83 & 11 & 913 & 0 & 이 & 0 \\
\hline F & A & $704-017 \mathrm{~F}$ & 768 & 71 & 7 & 497 & 0 & 0 & 0 \\
\hline $\mathbf{F}$ & A & $704-018 \mathrm{~F}$ & 1792 & 166 & 5 & 830 & 0 & 0 & 0 \\
\hline$F$ & A & $704-019 \mathrm{~F}$ & 1792 & 166 & 5 & 830 & 0 & 0 & 0 \\
\hline$F$ & A & $704-023 \mathrm{~F}$ & 1792 & 166 & 61 & 996 & 0 & 0 & 0 \\
\hline$F$ & A & 705-001F & 600 & 56 & 6 & 336 & 0 & 0 & 0 \\
\hline$F$ & A & $705-002 \mathrm{~F}$ & 600 & 56 & 6 & 336 & 0 & 0 & 0 \\
\hline$F$ & A & $706-000 \mathrm{~F}$ & 4001 & 372 & $37 \mid$ & 13764 & 0 & 0 & 0 \\
\hline$F$ & A & 707-001F & 3888 & 361 & 38 & 3718 & 2 & 0 & 2 \\
\hline$F$ & A & $707-002 \mathrm{~F}$ & 1484 & 138 & 91 & 1242 & 0 & 0 & 0 \\
\hline$F$ & A & $707-004 \mathrm{~F}$ & 240 & 22 & 9 & 198 & 0 & 0 & 0 \\
\hline$F$ & $\mathrm{~A}$ & 709-000F & 2075 & 193 & 40 & 7720 & 0 & 0 & 0 \\
\hline$F$ & A & $709-001 \mathrm{~F}$ & 2506 & 233 & 20 & 4660 & 0 & 0 & 0 \\
\hline $\mathbf{F}$ & $\mathrm{A}$ & 709-002F & 156 & 14 & 20 & 280 & 0 & 0 & 0 \\
\hline$F$ & $\mathrm{~A}$ & $709-004 \mathrm{~F}$ & 960 & 89 & 9 & 801 & 0 & 0 & $\overline{0}$ \\
\hline$F$ & A & 709-005F & 960 & 89 & 9 & 801 & 0 & 0 & 0 \\
\hline$F$ & $A$ & $711-000 \mathrm{~F}$ & 680 & 63 & 9 & 567 & 0 & 0 & 0 \\
\hline$F$ & $\mathbf{A}$ & $717-000 \mathrm{~F}$ & 52500 & 4877 & 37 & 130449 & 0 & 0 & 0 \\
\hline $\mathbf{F}$ & A & $717-004 \mathrm{~F}$ & 240 & 22 & 12 & 264 & 0 & 0 & 0 \\
\hline$F$ & A & $717-005 F$ & 100 & 9 & 12 & 108 & 0 & 0 & 0 \\
\hline $\mathbf{F}$ & A & $719-000 \mathrm{~F}$ & 192 & 18 & 31 & 558 & 0 & 0 & 0 \\
\hline$F$ & A & $722-000 \mathrm{~F}$ & 600 & 561 & 31 & 1736 & 0 & 0 & 0 \\
\hline$F$ & $\mathrm{~A}$ & $723-000 \mathrm{~F}$ & 12599 & 1170 & 40 & 46800 & 12 & 0 & 12 \\
\hline
\end{tabular}


Table A-5.--Incipient fire data for 700F Area

\begin{tabular}{|c|c|c|c|c|c|c|c|c|c|}
\hline \multirow[b]{2}{*}{ Area } & \multirow[b]{2}{*}{ Type } & \multirow[b]{2}{*}{ ID } & \multirow[b]{2}{*}{ Area, $\mathrm{ft}^{2}$} & \multirow[b]{2}{*}{ Area, $\mathbf{m}^{2}$} & \multirow{2}{*}{$\begin{array}{c}\text { years in } \\
\text { service to } \\
.94\end{array}$} & \multirow[b]{2}{*}{$\mathrm{yrs}^{*} \mathrm{~m}^{2}$} & \multicolumn{3}{|c|}{ Fire count } \\
\hline & & & & & & & $\begin{array}{c}\text { Original } \\
\text { count }\end{array}$ & '91 to '94 & $\begin{array}{l}\text { Sum of } \\
\text { all fires }\end{array}$ \\
\hline F & A & $723-001 \mathrm{~F}$ & 240 & 22 & 14 & 308 & 0 & 0 & 0 \\
\hline $\mathbf{F}$ & $\bar{A}$ & $723-002 F$ & 144 & 13 & 9 & 117 & $\overline{0}$ & $\overline{0}$ & $\overrightarrow{0}$ \\
\hline$F$ & $\bar{A}$ & $725-000 \mathrm{~F}$ & 35 & 3 & 27 & 81 & 0 & 0 & 0 \\
\hline $\mathbf{F}$ & $\mathbf{A}$ & $727-000 \mathrm{~F}$ & 240 & 22 & 9 & 198 & 0 & 0 & $\overline{0}$ \\
\hline F & A & $728-000 \mathrm{~F}$ & 3888 & 361 & 40 & 14440 & 0 & 0 & 0 \\
\hline F & A & $729-000 F$ & 3208 & 298 & 15 & 4470 & $\overline{0}$ & 0 & $\overline{0}$ \\
\hline F & A & $729-001 \mathrm{~F}$ & 384 & 36 & 9 & 324 & 0 & 0 & 0 \\
\hline F & A & $772-000 \mathrm{~F}$ & 83796 & 7785 & 40 & 311400 & 23 & 1 & 24 \\
\hline F & A & $772-001 \mathrm{~F}$ & 32000 & 2973 & 9 & 26757 & 1 & 0 & 1 \\
\hline $\mathbf{F}$ & $\bar{A}$ & $772-003 \mathrm{~F}$ & 1792 & 166 & 6 & 996 & $\overline{0}$ & 0 & $\overline{0}$ \\
\hline $\mathbf{F}$ & A & $782-001 \mathrm{~F}$ & 320 & 30 & 11 & 330 & 0 & 0 & 0 \\
\hline
\end{tabular}


Table A-6.--Incipient fire data for $700 \mathrm{H}$ Area

\begin{tabular}{|c|c|c|c|c|c|c|c|c|c|}
\hline \multirow[b]{2}{*}{ Area } & \multirow[b]{2}{*}{ Type } & \multirow[b]{2}{*}{ ID } & \multirow[b]{2}{*}{ Area, $\mathrm{ft}^{2}$} & \multirow[b]{2}{*}{ Area, $\mathbf{m}^{2}$} & \multirow{2}{*}{\begin{tabular}{|c} 
years in \\
service to \\
94
\end{tabular}} & \multirow[b]{2}{*}{$\mathrm{yrs}^{11} \mathbf{m}^{2}$} & \multicolumn{3}{|c|}{ Fire count } \\
\hline & & & & & & & $\begin{array}{c}\text { Original } \\
\text { count }\end{array}$ & '91 to '94 & $\begin{array}{l}\text { Sum of } \\
\text { all fires }\end{array}$ \\
\hline & & WTS & 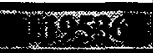 & 710 & & Why & $\sqrt{3}$ & & 2 \\
\hline $\mathrm{H}$ & A & $322-000 \mathrm{H}$ & 0 & 0 & 0 & 0 & 1 & 0 & 1 \\
\hline $\mathrm{H}$ & A & $701-001 \mathrm{H}$ & 4293 & 399 & 39 & 5561 & 1 & 0 & 1 \\
\hline $\mathrm{H}$ & A & $701-003 \mathrm{H}$ & 316 & 29 & 9 & 261 & 1 & $\overline{0}$ & 1 \\
\hline $\mathrm{H}$ & A & $701-004 \mathrm{H}$ & 65 & 6 & 9 & 54 & $\overline{0}$ & 0 & 0 \\
\hline $\bar{H}$ & A & $701-008 \mathrm{H}$ & 80 & $\overline{7}$ & 9 & 63 & $\overline{0}$ & $\overline{0}$ & 0 \\
\hline$\overline{\mathrm{H}}$ & $\bar{A}$ & $702-000 \mathrm{H}$ & 1665 & 155 & 10 & 1550 & 0 & $\overline{0}$ & 0 \\
\hline $\mathrm{H}$ & $A$ & $703-000 \mathrm{H}$ & 25000 & 2323 & 10 & 23230 & $\overline{0}$ & 1 & 1 \\
\hline $\mathrm{H}$ & A & $704-000 \mathrm{H}$ & 15000 & 1394 & 39 & $\$ 4366$ & 2 & 2 & 4 \\
\hline $\mathrm{H}$ & A & $704-001 \mathrm{H}$ & 1536 & 143 & 9 & 1287 & 0 & $\overline{0}$ & 0 \\
\hline $\bar{H}$ & $\mathbf{A}$ & $704-002 \mathrm{H}$ & 1536 & 143 & 9 & 1287 & 0 & $\overline{0}$ & 0 \\
\hline $\mathrm{H}$ & A & $704-003 \mathrm{H}$ & 1536 & 143 & 9 & 1287 & 0 & $\overline{0}$ & 0 \\
\hline $\mathrm{H}$ & A & $704-005 \mathrm{H}$ & 1536 & 143 & 9 & 1287 & 0 & $\underline{0}$ & 0 \\
\hline $\mathrm{H}$ & A & $704-006 \mathrm{H}$ & 7200 & 669 & 9 & 6021 & 0 & 0 & 0 \\
\hline $\mathrm{H}$ & A & $704-008 \mathrm{H}$ & 5040 & 468 & 13 & 6084 & 0 & 0 & 0 \\
\hline $\mathrm{H}$ & $\mathbf{A}$ & $704-011 \mathrm{H}$ & 240 & 22 & 9 & 198 & 0 & 0 & 0 \\
\hline $\mathrm{H}$ & A & $705-000 \mathrm{H}$ & 20000 & 1858 & 9 & 6722 & 0 & 2 & 2 \\
\hline $\mathrm{H}$ & $\mathbf{A}$ & $705-001 \mathrm{H}$ & 600 & 56 & 14. & 784 & 0 & 0 & 0 \\
\hline $\mathrm{H}$ & A & $705-002 \mathrm{H}$ & 600 & 56 & 14 & 784 & 0 & $\underline{0}$ & 0 \\
\hline$\vec{H}$ & $\mathrm{~A}$ & $705-005 \mathrm{H}$ & 960 & 89 & 9 & 801 & $\bar{c}$ & $\overline{0}$ & 0 \\
\hline $\mathrm{H}$ & A & 706-000H & 4220 & 392 & 39 & 5288 & c & $\overline{0}$ & 0 \\
\hline$\overline{\mathrm{H}}$ & $\bar{A}$ & $706-001 \mathrm{H}$ & 1536 & 143 & 9 & 1287 & $\mathrm{c}$ & 0 & 70 \\
\hline$\overline{\mathrm{H}}$ & A & $706-002 \mathrm{H}$ & 1536 & 143 & 14 & 2002 & $\mathrm{c}$ & 0 & 0 \\
\hline $\mathrm{H}$ & $\bar{A}$ & $707-000 \mathrm{H}$ & 19000 & 1765 & 9 & 1.5885 & $\mathrm{c}$ & 0 & 7 \\
\hline $\mathrm{H}$ & $\bar{A}$ & $717-003 \mathrm{H}$ & 360 & 33 & 15 & 495 & $\mathrm{c}$ & 0 & $\mathrm{~T}$ \\
\hline $\mathrm{H}$ & $\mathrm{A}$ & $717-004 \mathrm{H}$ & 360 & 33 & 15 & 495 & $\mathrm{c}$ & $\underline{0}$ & $\underline{0}$ \\
\hline $\mathrm{H}$ & A & $724-000 \mathrm{H}$ & 5001 & 465 & 39 & 8135 & 1 & 0 & 1 \\
\hline$\overline{\mathrm{H}}$ & $\mathrm{A}$ & $777-000 \mathrm{H}$ & 0 & 0 & 0 & $\bar{\Omega}$ & 1 & 0 & 1 \\
\hline $\mathrm{H}$ & A & $782-001 \mathrm{H}$ & 320 & 30 & 11 & 330 & ( & 0 & 0 \\
\hline
\end{tabular}


Table A-7.--Incipient fire data for 700K Area

\begin{tabular}{|c|c|c|c|c|c|c|c|c|c|}
\hline \multirow[b]{2}{*}{ Area } & \multirow[b]{2}{*}{ Type } & \multirow[b]{2}{*}{ ID } & \multirow[b]{2}{*}{ Area, $\mathbf{f t}^{2}$} & \multirow[b]{2}{*}{ Area, $\mathbf{m}^{2}$} & \multirow{2}{*}{$\begin{array}{c}\text { years in } \\
\text { service to } \\
94\end{array}$} & \multirow[b]{2}{*}{$\operatorname{yrs}^{*} m^{2}$} & \multicolumn{3}{|c|}{ Fire count } \\
\hline & & & & & & & $\begin{array}{l}\text { Original } \\
\text { count }\end{array}$ & '91 to '94 & $\begin{array}{l}\text { Sum of } \\
\text { all fires }\end{array}$ \\
\hline & & MY & Tre & 85 & & गुस: & & 3 & 5 \\
\hline $\mathbf{K}$ & A & $701-001 \mathrm{~K}$ & 4162 & 387 & 40 & 15480 & 1 & 0 & 1 \\
\hline $\bar{K}$ & A & $701-002 \mathrm{~K}$ & 1679 & 156 & 7 & 1092 & $\overline{0}$ & 0 & 0 \\
\hline$\overline{\mathbf{K}}$ & $\bar{A}$ & $701-005 K$ & 308 & 29 & 7 & 203 & $\overline{0}$ & $\overline{0}$ & 0 \\
\hline $\mathbf{K}$ & $A$ & $704-000 \mathrm{~K}$ & 15870 & 1474 & 40 & 58960 & 0 & 0 & 0 \\
\hline $\mathbf{K}$ & A & 704-001K & 1792 & 166 & 12 & 1992 & 0 & 0 & 0 \\
\hline $\mathbf{K}$ & $\mathbf{A}$ & $704-002 \mathrm{~K}$ & 845 & 79 & 5 & 395 & 0 & 0 & 0 \\
\hline$\overline{\mathbf{K}}$ & A & $704-003 \mathrm{~K}$ & 845 & 79 & 5 & 395 & 0 & 0 & 0 \\
\hline $\bar{K}$ & A & $704-004 \mathrm{~K}$ & 845 & 79 & 5 & 395 & 0 & $\overline{0}$ & 0 \\
\hline$\overline{\mathbf{K}}$ & $\bar{A}$ & $704-022 \mathrm{~K}$ & 1680 & 156 & 4 & 624 & $\overline{0}$ & $\overline{1}$ & 1 \\
\hline$\overline{\mathbf{K}}$ & $\bar{A}$ & $711-001 \mathrm{~K}$ & 800 & 74 & 5 & 370 & $\overline{0}$ & $\overline{0}$ & 0 \\
\hline$\overline{\mathbf{K}}$ & $\mathbf{A}$ & $717-000 \mathrm{~K}$ & 20000 & 1858 & 6 & 11148 & $\overline{0}$ & 1 & 1 \\
\hline
\end{tabular}


Table A-8.--Incipient fire data for $700 \mathrm{~L}$ Area

\begin{tabular}{|c|c|c|c|c|c|c|c|c|c|}
\hline \multirow[b]{2}{*}{ Area } & \multirow[b]{2}{*}{ Type } & \multirow[b]{2}{*}{ ID } & \multirow[b]{2}{*}{ Area, $\mathrm{ft}^{2}$} & \multirow[b]{2}{*}{ Area, $\mathbf{m}^{2}$} & \multirow{2}{*}{$\left|\begin{array}{c}\text { years in } \\
\text { service to } \\
94\end{array}\right|$} & \multirow[b]{2}{*}{$\mathrm{yrs}^{*} \mathrm{~m}^{2}$} & \multicolumn{3}{|c|}{ Fire count } \\
\hline & & & & & & & $\begin{array}{l}\text { Original } \\
\text { count }\end{array}$ & |'91 to '94 & $\begin{array}{l}\text { Sum of } \\
\text { all fires }\end{array}$ \\
\hline & & का & Rats & 75 & $T$ & TIII & & $\pi$ & 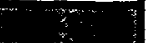 \\
\hline L & $\mathrm{A}$ & $701-001 L$ & 228 & 21 & 401 & 840 & 0 & 0 & 0 \\
\hline $\mathrm{L}$ & A & $701-002 \mathrm{~L}$ & 1679 & 156 & 7 & 1092 & 2 & 0 & 2 \\
\hline$L$ & $\mathrm{~A}$ & $701-005 \mathrm{~L}$ & 308 & 29 & 7 & 203 & $\overline{0}$ & 0 & 0 \\
\hline $\mathrm{L}$ & $\mathbf{A}$ & $704-000 \mathrm{~L}$ & 14487 & 1346 & 40 & 53840 & 1 & 0 & 1 \\
\hline L & $\bar{A}$ & $704-001 \mathrm{~L}$ & 1792 & 166 & 81 & 1328 & $\overline{0}$ & $\overline{0}$ & 0 \\
\hline $\mathrm{L}$ & A & $711-000 \mathrm{~L}$ & 630 & 59 & 40 & 2360 & 0 & 0 & 0 \\
\hline $\mathrm{L}$ & A & $717-000 \mathrm{~L}$ & 0 & 0 & 0 & 0 & 1 & 0 & 1 \\
\hline $\mathrm{L}$ & A & $723-000 \mathrm{~L}$ & 180 & 17 & 5] & 85 & 0 & 0 & 0 \\
\hline $\mathrm{L}$ & $\mathrm{A}$ & $723-001 \mathrm{~L}$ & 85 & 8 & 5 & 40 & 0 & 0 & 0 \\
\hline $\bar{L}$ & $\bar{A}$ & $723-002 \mathrm{~L}$ & 80 & 7 & 5 & 35 & 0 & 0 & 0 \\
\hline $\bar{L}$ & $\mathbf{A}$ & $723.003 \mathrm{~L}$ & 80 & 7 & 5 & 35 & 0 & $\overline{0}$ & $\overline{0}$ \\
\hline $\mathrm{L}$ & $\mathrm{A}$ & $723-004 \mathrm{~L}$ & 200 & 19 & 8 & 152 & $\overline{0}$ & 0 & 0 \\
\hline & & & (18s & 20 & & $\Sigma_{2-1}$ & & & 5 \\
\hline
\end{tabular}


Table A-9.--Incipient fire data for 700M Area

\begin{tabular}{|c|c|c|c|c|c|c|c|c|c|}
\hline \multirow[b]{2}{*}{ Area } & \multirow[b]{2}{*}{ Type } & \multirow[b]{2}{*}{ ID } & \multirow[b]{2}{*}{ Area, $\mathrm{ft}^{2}$} & \multirow[b]{2}{*}{ Area, $\mathbf{m}^{2}$} & \multirow{2}{*}{$\begin{array}{c}\text { years in } \\
\text { service to } \\
94\end{array}$} & \multirow[b]{2}{*}{ yrs ${ }^{*} m^{2}$} & \multicolumn{3}{|c|}{ Fire count } \\
\hline & & & & & & & $\begin{array}{c}\text { Origina } \\
\text { count }\end{array}$ & '91 to 94 & $\begin{array}{l}\text { Sum of } \\
\text { all fires }\end{array}$ \\
\hline & & 91 & 38 & 51 & & TTS & & 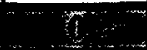 & $x$ \\
\hline $\mathbf{M}$ & A & 701-001M & 315 & 29 & 42 & 1218 & 0 & 0 & 0 \\
\hline $\mathbf{M}$ & $\mathrm{A}$ & $701-003 \mathrm{M}$ & 195 & 18 & 42 & 756 & $\overline{0}$ & 0 & 0 \\
\hline $\mathbf{M}$ & A & $701-004 \mathrm{M}$ & 192 & 18 & 4 & 72 & 1 & 0 & 1 \\
\hline $\mathbf{M}$ & $\mathrm{A}$ & $701-006 \mathrm{M}$ & 80 & 7 & 42 & 294 & 1 & $\overline{0}$ & 1 \\
\hline$\overline{\mathbf{M}}$ & $\mathrm{A}$ & $701-007 \mathrm{M}$ & 420 & 39 & 4 & 156 & $\overline{0}$ & $\overline{0}$ & $\overrightarrow{0}$ \\
\hline $\mathbf{M}$ & A & 704-000M & 7672 & 713 & 42 & 29946 & 0 & 0 & 0 \\
\hline $\mathbf{M}$ & A & $710-000 \mathrm{M}$ & 250 & 23 & 42 & 966 & 0 & 0 & 0 \\
\hline $\mathbf{M}$ & A & $730-000 \mathrm{M}$ & 18000 & 1672 & 9 & 15048 & 0 & 0 & 0 \\
\hline $\bar{M}$ & $\mathbf{A}$ & $734-000 \mathrm{M}$ & 450 & 42 & 9 & 378 & $\overline{0}$ & $\overline{0}$ & 0 \\
\hline$\overline{\mathbf{M}}$ & $\mathbf{A}$ & $777-000 \mathrm{M}$ & $\overline{0}$ & $\overline{0}$ & 0 & $\overline{0}$ & $\overline{3}$ & $\overline{0}$ & 3 \\
\hline $\bar{M}$ & A & $782-001 \mathrm{M}$ & 320 & 30 & 12 & 360 & $\overline{0}$ & $\overline{0}$ & 0 \\
\hline
\end{tabular}


Table A-10.--Incipient fire data for 700P Area

\begin{tabular}{|c|c|c|c|c|c|c|c|c|c|}
\hline \multirow[b]{2}{*}{ Area } & \multirow[b]{2}{*}{ Type } & \multirow[b]{2}{*}{ ID } & \multirow[b]{2}{*}{ Area, $\mathrm{ft}^{2}$} & \multirow[b]{2}{*}{ Area, $\mathbf{m}^{2}$} & \multirow{2}{*}{$\begin{array}{c}\text { years in } \\
\text { service to } \\
94\end{array}$} & \multirow[b]{2}{*}{$y r{ }^{*} \mathbf{n}^{2}$} & \multicolumn{3}{|c|}{ Fire count } \\
\hline & & & & & & & $\begin{array}{c}\text { Original } \\
\text { count }\end{array}$ & '91 to $94 \mid$ & $\begin{array}{l}\text { Sum of } \\
\text { all fires }\end{array}$ \\
\hline & & STs & $7 \times 3$ & 78 & & me & 5 & i: & 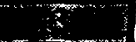 \\
\hline $\mathbf{P}$ & A & $701-001 P$ & 4164 & 387 & 40 & 1.5480 & 0 & 이 & 0 \\
\hline $\mathbf{P}$ & $\bar{A}$ & $701-002 P$ & 1679 & 156 & 7 & 1092 & 1 & of & 1 \\
\hline $\mathbf{P}$ & $\mathrm{A}$ & 701-005P & 308 & 29 & 7 & 203 & 0 & 0] & 0 \\
\hline $\bar{P}$ & $\bar{A}$ & 704-002P & 870 & 81 & 7 & 567 & 0 & 0 & 0 \\
\hline $\bar{P}$ & $\mathbf{A}$ & $704-003 P$ & 896 & 83 & 7 & 581 & 0 & 0 & 0 \\
\hline$\overline{\mathbf{P}}$ & $\bar{A}$ & $704-000 \mathrm{P}$ & 15870 & 1474 & 40 & 53960 & 2 & 0 & 2 \\
\hline $\mathbf{P}$ & $\bar{A}$ & $711-000 \mathrm{P}$ & 630 & $\overline{59}$ & 40 & 2360 & 0 & 0 & -1 \\
\hline & & & & & & & & & \\
\hline
\end{tabular}


Table A-11.--Incipient fire data for 700R Area

\begin{tabular}{|c|c|c|c|c|c|c|c|c|c|}
\hline \multirow[b]{2}{*}{ Area } & \multirow[b]{2}{*}{ Type } & \multirow[b]{2}{*}{ ID } & \multirow[b]{2}{*}{ Area, $\mathbf{f t}^{2}$} & \multirow[b]{2}{*}{ Area, $\mathrm{m}^{2}$} & \multirow{2}{*}{$\begin{array}{c}\text { years in } \\
\text { service to } \\
' 94\end{array}$} & \multirow[b]{2}{*}{$\mathrm{yrs}^{*} \mathrm{~m}^{2}$} & \multicolumn{3}{|c|}{ Fire count } \\
\hline & & & & & & & $\begin{array}{c}\text { Original } \\
\text { count }\end{array}$ & '91 to '94 & $\begin{array}{l}\text { Sum of } \\
\text { all fires }\end{array}$ \\
\hline & & 7 & $\tan$ & 1 & & bis & & 5 & \\
\hline $\mathbf{R}$ & A & $701-1 R$ & 4162 & 387 & 7 & 2709 & 0 & 0 & 0 \\
\hline $\mathbf{R}$ & $\mathbf{A}$ & $701-2 \mathrm{R}$ & 150 & 14 & 7 & 98 & 0 & 0 & 0 \\
\hline$\overline{\mathbf{R}}$ & $\bar{A}$ & $701-3 R$ & 64 & 6 & 7 & 42 & 0 & $\overline{0}$ & 0 \\
\hline $\mathbf{R}$ & A & 704-R & 15870 & 1474 & 41 & 60434 & 1 & 0 & 1 \\
\hline $\mathbf{R}$ & A & $711-R$ & 630 & 59 & 7 & 413 & 0 & 0 & 0 \\
\hline
\end{tabular}


Table A-12.--Incipient fire data for 100 Area

\begin{tabular}{|c|c|c|c|c|c|c|c|c|c|}
\hline \multirow[b]{2}{*}{ Area } & \multirow[b]{2}{*}{ Type } & \multirow[b]{2}{*}{ ID } & \multirow[b]{2}{*}{ Area, $\mathrm{ft}^{2}$} & \multirow[b]{2}{*}{ Area, $\mathrm{m}^{2}$} & \multirow{2}{*}{\begin{tabular}{|c|} 
years in \\
service to \\
94
\end{tabular}} & \multirow[b]{2}{*}{$\mathrm{yrs}^{*} \dot{m}^{2}$} & \multicolumn{3}{|c|}{ Fire count } \\
\hline & & & & & & & $\begin{array}{c}\text { Original } \\
\text { count }\end{array}$ & '91 to '94 & $\begin{array}{l}\text { Sum of } \\
\text { all fires }\end{array}$ \\
\hline & & min & 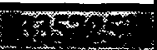 & ning & & THa & $7 x$ & 4 & $\pi$ \\
\hline C & $P$ & $105-000 \mathrm{C}$ & 500000 & 46452 & 37 & 1718724 & 17 & 1 & 18 \\
\hline $\bar{C}$ & $\mathrm{P}$ & $105.006 \mathrm{C}$ & 240 & $\overline{22}$ & 9 & 198 & 0 & 0 & 0 \\
\hline C & $\mathrm{P}$ & $105-007 \mathrm{C}$ & 240 & 22 & 9 & 198 & 0 & 0 & $\overrightarrow{0}$ \\
\hline $\mathrm{C}$ & $\mathrm{P}$ & $108-001 \mathrm{C}$ & 6000 & 557 & 33 & 18381 & 0 & 0 & 0 \\
\hline C & P & $108-002 \mathrm{C}$ & 6000 & 557 & 33 & 18381 & 0 & 0 & 0 \\
\hline C & $\mathbf{P}$ & $110-000 \mathrm{C}$ & 240 & $\overline{22}$ & 37 & 814 & 0 & 0 & 0 \\
\hline C & $\mathrm{P}$ & $151-001 \mathrm{C}$ & 6400 & 595 & 33 & 9635 & 0 & 0 & 0 \\
\hline C & $\mathbf{P}$ & $151-002 \mathrm{C}$ & 6400 & 595 & 33 & 9635 & 0 & 0 & 0 \\
\hline C & $\mathbf{P}$ & $152-007 \mathrm{C}$ & 200 & 19 & 7 & 133 & 0 & 0 & 0 \\
\hline C & P & $183-002 \mathrm{C}$ & 760 & 71 & 39 & 2769 & 0 & 0 & 0 \\
\hline C & $\mathrm{P}$ & $183-004 \mathrm{C}$ & 6430 & 597 & 39 & 13283 & 0 & 0 & 0 \\
\hline C & $\mathrm{P}$ & $184-002 \mathrm{C}$ & 828 & 77 & 39 & 3003 & 0 & 0 & 0 \\
\hline $\mathrm{C}$ & $P$ & $184-006 \mathrm{C}$ & 800 & 74 & 7 & 518 & 0 & 0 & 0 \\
\hline C & $\mathbf{P}$ & $190-000 \mathrm{C}$ & 12000 & 1115 & 37 & 41255 & 0 & 0 & 0 \\
\hline C & P & $191-000 \mathrm{C}$ & 288 & 27 & 37 & 999 & 0 & 0 & $\overline{0}$ \\
\hline $\mathbf{K}$ & $\mathrm{P}$ & $105-000 \mathrm{~K}$ & 500000 & 46452 & 37 & 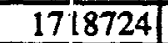 & 8 & 7 & 15 \\
\hline $\mathbf{K}$ & $\mathbf{P}$ & $105-006 \mathrm{~K}$ & 96 & 9 & 7 & 63 & 0 & 0 & 0 \\
\hline $\mathbf{K}$ & $\mathrm{P}$ & $105-007 \mathrm{~K}$ & 96 & 9 & 7 & 63 & 0 & 0 & 0 \\
\hline $\mathbf{K}$ & $\mathbf{P}$ & $105-008 \mathrm{~K}$ & 120 & 11 & 7 & 77 & 0 & 0 & 0 \\
\hline $\bar{K}$ & $\bar{P}$ & $105-013 \mathrm{~K}$ & 3250 & 302 & 7 & 2114 & 0 & 0 & 0 \\
\hline $\mathbf{K}$ & $\mathbf{P}$ & $108-001 \mathrm{~K}$ & 6000 & 557 & 40 & 22280 & 3 & $\overline{0}$ & 3 \\
\hline $\mathrm{K}$ & $\mathbf{P}$ & $108-002 \mathrm{~K}$ & 6000 & 557 & 40 & 22280 & 1 & 0 & 1 \\
\hline $\mathbf{K}$ & $\bar{P}$ & $110-000 \mathrm{~K}$ & 240 & 22 & 40 & 880 & 0 & 0 & 0 \\
\hline $\bar{K}$ & $\mathbf{P}$ & $151-001 \mathrm{~K}$ & 6400 & 595 & 37 & 22015 & 0 & 0 & 0 \\
\hline$\overline{\mathbf{K}}$ & P & $151-002 \mathrm{~K}$ & 6400 & 595 & 37 & 22015 & 0 & 0 & 0 \\
\hline $\mathbf{K}$ & P & $152-001 \mathrm{~K}$ & 6400 & 595 & 37 & 22015 & 0 & 0 & 0 \\
\hline$\overline{\mathbf{K}}$ & $\mathrm{P}$ & $152-007 \mathrm{~K}$ & 200 & 19 & 9 & 171 & 0 & 0 & 0 \\
\hline$\overline{\mathbf{K}}$ & $\mathbf{P}$ & $183-002 \mathrm{~K}$ & 720 & 67 & 40 & 2680 & 1 & 0 & 1 \\
\hline$\overline{\mathbf{K}}$ & $P$ & $183-004 \mathrm{~K}$ & 6430 & 597 & 40 & 23880 & 0 & 1 & 1 \\
\hline $\mathbf{K}$ & $P$ & $184-000 \mathrm{~K}$ & 85000 & 7897 & 37 & 292189 & 11 & 0 & 11 \\
\hline $\mathbf{K}$ & $\mathrm{P}$ & $184-005 \mathrm{~K}$ & 173 & 16 & 9 & 144 & 0 & 0 & 0 \\
\hline $\mathbf{K}$ & $\mathrm{P}$ & $185-000 \mathrm{~K}$ & 375 & 35 & 40 & 1400 & 1 & 0 & 1 \\
\hline $\mathbf{K}$ & $\mathbf{P}$ & $190-000 \mathrm{~K}$ & 12000 & 1115 & 37 & 41255 & 0 & 0 & 0 \\
\hline $\mathbf{K}$ & P & $191-000 \mathrm{~K}$ & 288 & 27 & 31 & 837 & 1 & 0 & 1 \\
\hline$L$ & $\mathbf{P}$ & $105-000 \mathrm{~L}$ & 500000 & 46452 & 37 & 1718724 & 3 & 0 & 3 \\
\hline $\mathrm{L}$ & $\mathbf{P}$ & $105-002 \mathrm{~L}$ & 720 & 67 & 9 & 603 & 0 & 0 & $\overrightarrow{0}$ \\
\hline $\bar{L}$ & $\mathbf{P}$ & $108-001 \mathrm{~L}$ & 6000 & 557 & 37 & 20609 & 2 & 0 & 2 \\
\hline $\bar{L}$ & $\mathbf{P}$ & $108-002 L$ & 6000 & 557 & 37 & 20609 & 0 & $\overline{0}$ & $\overline{0}$ \\
\hline$\overline{\mathrm{L}}$ & $\bar{P}$ & 108-004L & 625 & 58 & 9 & 522 & 1 & $\overline{0}$ & 1 \\
\hline $\mathrm{L}$ & $\mathbf{P}$ & $110-000 \mathrm{~L}$ & 240 & 22 & 37. & 814 & 0 & 0 & 0 \\
\hline $\mathrm{L}$ & $\mathbf{P}$ & $151-001 \mathrm{~L}$ & 6400 & 595 & 37 & 22015 & 0 & 0 & 0 \\
\hline $\mathrm{L}$ & $\mathbf{P}$ & $151-002 \mathrm{~L}$ & 6400 & 595 & 37 & 22015 & 0 & 0 & 0 \\
\hline$\overline{\mathrm{L}}$ & $\mathbf{P}$ & $152-001 \mathrm{~L}$ & 6400 & 595 & 37 & 22015 & 0 & 0 & 0 \\
\hline $\bar{L}$ & $\mathbf{P}$ & $152-007 \mathrm{~L}$ & 200 & 19 & 7 & 133 & 0 & 0 & 0 \\
\hline $\mathrm{L}$ & $\bar{P}$ & $183-002 \mathrm{~L}$ & 720 & 67. & 40 & 2680 & 0 & 0 & 0 \\
\hline $\mathrm{L}$ & $\mathrm{P}$ & $183-004 \mathrm{~L}$ & 6430 & 597 & 40 & 23880 & 0 & 0 & 0 \\
\hline
\end{tabular}


Table A-12.--Incipient fire data for 100 Area

\begin{tabular}{|c|c|c|c|c|c|c|c|c|c|}
\hline \multirow[b]{2}{*}{ Area } & \multirow[b]{2}{*}{ Type } & \multirow[b]{2}{*}{ ID } & \multirow[b]{2}{*}{ Area, $f^{2}$} & \multirow[b]{2}{*}{ Area, $\mathrm{m}^{2}$} & \multirow{2}{*}{\begin{tabular}{|c|} 
years in \\
service to \\
94
\end{tabular}} & \multirow[b]{2}{*}{$\mathrm{yrs}^{*} \mathrm{~m}^{2}$} & \multicolumn{3}{|c|}{ Fire count } \\
\hline & & & & & & & $\begin{array}{c}\text { Original } \\
\text { count }\end{array}$ & $\mid$ '91 to '94| & $\begin{array}{l}\text { Sum of } \\
\text { all fires }\end{array}$ \\
\hline$L$ & $\mathbf{P}$ & 184-000L & 85000 & 7897 & 37 & 292189 & 3 & 0 & 3 \\
\hline $\mathbf{L}$ & $\mathbf{P}$ & $184-006 \mathrm{~L}$ & 800 & 74 & 7 & 518 & 0 & 0 & 0 \\
\hline$L$ & $\mathbf{P}$ & $190-000 \mathrm{~L}$ & 12000 & 1115 & 37 & 41255 & 0 & 0 & 0 \\
\hline $\mathrm{L}$ & $\mathbf{P}$ & $191.000 \mathrm{~L}$ & 288 & 27 & 30 & 810 & 0 & of & 0 \\
\hline $\mathbf{P}$ & $\mathrm{P}$ & $105-000 \mathrm{P}$ & 750000 & 69677 & 37 & 2578049 & 13 & 2 & 15 \\
\hline $\mathbf{P}$ & $\mathbf{P}$ & $105-002 \mathrm{P}$ & 720 & 67 & 7 & 469 & 0 & o & 0 \\
\hline $\mathbf{P}$ & $\mathbf{P}$ & $105-003 \mathrm{P}$ & 720 & 67 & 7 & 469 & 0 & 0 & $\overline{0}$ \\
\hline $\mathbf{P}$ & $\mathbf{P}$ & $105-006 \mathrm{P}$ & 96 & 9 & 7 & 63 & 0 & 0 & $\overline{0}$ \\
\hline $\mathbf{P}$ & $\mathbf{P}$ & $105-013 P$ & 3250 & 302 & 7 & 2114 & 0 & 0 & 0 \\
\hline $\mathbf{P}$ & P & $105-014 \mathrm{P}$ & 360 & 33 & 8 & 264 & 0 & 0 & $\overline{0}$ \\
\hline $\mathbf{P}$ & $\mathbf{P}$ & 108-001P & 6000 & 557 & 40 & 22280 & 1 & ol & 1 \\
\hline $\mathbf{P}$ & P & $108-002 \mathrm{P}$ & 6000 & 557 & 40 & 22280 & 2 & 0 & 2 \\
\hline $\mathbf{P}$ & $\mathbf{P}$ & $110-000 \mathrm{P}$ & 240 & 22 & 40 & 880 & $\overline{0}$ & 0 & $\overline{0}$ \\
\hline $\mathbf{P}$ & $\bar{P}$ & $151-001 \mathrm{P}$ & 6400 & 595 & 37 & 22015 & $\overline{0}$ & 0 & $\overline{0}$ \\
\hline $\mathbf{P}$ & $\mathbf{P}$ & $151.002 \mathrm{P}$ & 6400 & 595 & 37 & 22015 & 0 & of & 0 \\
\hline $\mathbf{P}$ & $\mathbf{P}$ & $152-001 P$ & 6400 & 595 & 37 & 22015 & 0 & of & $\overline{0}$ \\
\hline $\mathbf{P}$ & $\mathbf{P}$ & $152-007 \mathrm{P}$ & 200 & 19 & 7 & 133 & 0 & 0 & 0 \\
\hline $\mathbf{P}$ & $\mathbf{P}$ & $183-002 \mathrm{P}$ & 720 & 67 & 40 & 2680 & 0 & 0 & 0 \\
\hline $\mathbf{P}$ & P & $183-004 \mathrm{P}$ & 6430 & 597 & 40 & 23880 & 1 & 0 & 1 \\
\hline P & $\mathrm{P}$ & 184-000P & 85000 & 7897 & 37 & 292189 & 12 & of & 12 \\
\hline $\mathbf{P}$ & $\mathbf{P}$ & 184-005P & 173 & 16 & 7 & 112 & 0 & 0 & 0 \\
\hline$\overline{\mathbf{P}}$ & $\mathbf{P}$ & 184-006P & 800 & 74 & 7 & 518 & 0 & 0 & 0 \\
\hline $\mathbf{P}$ & P & $185-000 \mathrm{P}$ & 375 & 35 & 40 & 1400 & 1 & 0 & 1 \\
\hline $\mathbf{P}$ & $\mathbf{P}$ & $190-000 \mathrm{P}$ & 12000 & 1115 & 37 & 41255 & 0 & of & 0 \\
\hline $\mathbf{P}$ & $\mathbf{P}$ & $191-000 \mathrm{P}$ & 288 & 27 & 30 & 810 & 0 & 0 & 0 \\
\hline $\mathbf{R}$ & P & $100-000 \mathrm{R}$ & 0 & 0 & 0 & 0 & 1 & of & 1 \\
\hline $\mathbf{R}$ & $P$ & $105-000 \mathrm{R}$ & 500000 & 46452 & 41 & 1904532 & 8 & 0 & 8 \\
\hline $\mathbf{R}$ & $\mathrm{P}$ & 108-001R & 6596 & 613 & 41 & 25133 & 0 & 0 & 0 \\
\hline $\mathbf{R}$ & $\mathbf{P}$ & $108-002 R$ & 9525 & 885 & 41 & 36285 & $\overline{0}$ & 0 & $\overline{0}$ \\
\hline $\mathbf{R}$ & $\mathbf{P}$ & $110-000 \mathrm{R}$ & 240 & 22 & 37 & 814 & 0 & 0 & 0 \\
\hline $\mathbf{R}$ & $\mathbf{P}$ & $122-000 \mathrm{R}$ & 4759 & 442 & 41 & 18122 & $\overline{0}$ & 0 & $\overline{0}$ \\
\hline $\mathbf{R}$ & P & $151-001 R$ & 6151 & 571 & 41 & 23411 & 0 & 0 & 0 \\
\hline $\mathbf{R}$ & $\bar{P}$ & $151-002 R$ & 7601 & 706 & 41 & 28946 & 0 & 0 & $\overline{0}$ \\
\hline $\mathbf{R}$ & P & $181-000 \mathrm{R}$ & 144 & 13 & 36 & 468 & 0 & 0 & $\overline{0}$ \\
\hline $\mathbf{R}$ & P & 183-001R & 2640 & 245 & 41 & 10045 & 0 & 0 & 0 \\
\hline $\mathbf{R}$ & $\mathbf{P}$ & $183-002 \mathrm{R}$ & 1150 & 107 & 41 & 4387 & 0 & 이 & 0 \\
\hline $\mathbf{R}$ & P & $184-000 \mathrm{R}$ & 85000 & 7897 & 37 & 292189 & 3 & 0 & 3 \\
\hline $\mathbf{R}$ & $\mathbf{P}$ & $190-000 R$ & 15638 & 1453 & 41 & 59573 & 1 & 0 & \\
\hline
\end{tabular}


Table A-13.--Incipient fire data for 400D Area

\begin{tabular}{|c|c|c|c|c|c|c|c|c|c|}
\hline \multirow[b]{2}{*}{ Area } & \multirow[b]{2}{*}{ Type } & \multirow[b]{2}{*}{ ID } & \multirow[b]{2}{*}{ Area, $\mathrm{ft}^{2}$} & \multirow[b]{2}{*}{ Area, $\mathbf{m}^{2}$} & \multirow{2}{*}{\begin{tabular}{|c|} 
years in \\
service to \\
$' 94$
\end{tabular}} & \multirow[b]{2}{*}{$y r s^{*} m^{2}$} & \multicolumn{3}{|c|}{ Fire count } \\
\hline & & & & & & & $\begin{array}{l}\text { Original } \\
\text { count }\end{array}$ & |'91 to '94| & $\begin{array}{l}\text { Sum of } \\
\text { all fires }\end{array}$ \\
\hline & & ता. & 6019 & 798: & & 12891 & 78 & $\mathrm{TH}$ & 78 \\
\hline D & $P$ & $400-000 D$ & 0 & 0 & 41 & 0 & 2 & 0) & 2 \\
\hline $\bar{D}$ & $\mathrm{P}$ & $401-001 D$ & 1930 & 179 & 41 & 7339 & 2 & 0 & 2 \\
\hline D & P & $401-002 \mathrm{D}$ & 300 & 28 & 41 & 1148 & 0 & 0 & 0 \\
\hline $\bar{D}$ & $\bar{P}$ & $402-000 \mathrm{D}$ & 7500 & 697 & 41 & 28577 & 1 & o & 1 \\
\hline $\mathrm{D}$ & $\bar{P}$ & $411-003 D$ & 7464 & 693 & 41 & $2 \varepsilon / 413$ & $\overline{2}$ & 0 & $\overline{2}$ \\
\hline $\bar{D}$ & P & $411-004 \mathrm{D}$ & 352 & 33 & 41 & 1353 & 0 & 0 & 0 \\
\hline D & P & $411-006 \mathrm{D}$ & 360 & 33 & 41 & 1353 & 0 & 0 & $\overline{0}$ \\
\hline $\mathrm{D}$ & $\mathbf{P}$ & $411-007 \mathrm{D}$ & 240 & 22 & 41 & 902 & 0 & 0 & $\overline{0}$ \\
\hline D & $\bar{P}$ & $412-001 \mathrm{D}$ & 1000 & 93 & 33 & 5069 & 33 & 0 & 33 \\
\hline $\bar{D}$ & $\overline{\mathbf{P}}$ & $412-011 \mathrm{D}$ & 100 & 9 & 41 & 369 & 0 & 0 & 0 \\
\hline $\bar{D}$ & $\overline{\mathbf{P}}$ & $412-002 \mathrm{D}$ & 2402 & 223 & 41 & 9143 & $\overline{0}$ & 0 & $\overline{0}$ \\
\hline $\bar{D}$ & $\overline{\mathbf{P}}$ & $412-003 D$ & 5790 & 538 & 41 & 22058 & $\overline{0}$ & 0 & $\overline{0}$ \\
\hline D & $\bar{P}$ & $412-004 \mathrm{D}$ & 1098 & 102 & 411 & $4 \longdiv { 1 8 2 }$ & $\overline{0}$ & 0 & $\overline{0}$ \\
\hline $\mathrm{D}$ & $\mathbf{P}$ & $412-005 D$ & 1200 & 111 & 41 & 4551 & 0 & 0 & 0 \\
\hline $\bar{D}$ & $\overline{\mathrm{P}}$ & $412-008 \mathrm{D}$ & 631 & 59 & 20 & 1180 & $\overline{0}$ & 0 & 0 \\
\hline $\mathrm{D}$ & $\overline{\mathrm{P}}$ & $412-009 \mathrm{D}$ & 366 & 34 & 41 & 1394 & $\overline{0}$ & 0 & $\overline{0}$ \\
\hline D & $\mathbf{P}$ & $413-000 \mathrm{D}$ & $\overline{0}$ & 0 & 33 & $\overline{0}$ & 8 & 0 & $\overline{8}$ \\
\hline D & $\mathbf{P}$ & 414-000D & 1200 & 111 & 41 & 4551 & 0 & 0 & $\overline{0}$ \\
\hline $\bar{D}$ & $\bar{P}$ & $415-000 \mathrm{D}$ & 1200 & 111 & 411 & 4551 & 0 & $\overline{0}$ & $\overline{0}$ \\
\hline $\bar{D}$ & $\bar{P}$ & $420-002 \mathrm{D}$ & 880 & 82 & 18 & 1476 & 0 & 0 & $\overline{0}$ \\
\hline D & $\overline{\mathbf{P}}$ & $420-0000$ & 6030 & 560 & 41 & 22960 & $\overline{0}$ & 0 & $\overline{0}$ \\
\hline $\bar{D}$ & $\bar{P}$ & $421-002 \mathrm{D}$ & 5525 & 513 & 40 & 20520 & $\overline{0}$ & 0 & $\overline{0}$ \\
\hline $\bar{D}$ & $\overline{\mathbf{P}}$ & $421.004 \mathrm{D}$ & 3600 & 334 & 13 & 4342 & $\overline{0}$ & $\overline{0}$ & $\overline{0}$ \\
\hline $\bar{D}$ & $\overline{\mathbf{P}}$ & $421-000 \mathrm{D}$ & 8128 & 755 & 41 & 30955 & $\overline{2}$ & $\overline{0}$ & $\overline{2}$ \\
\hline $\bar{D}$ & $\overline{\mathbf{P}}$ & $451-000 \mathrm{D}$ & 566 & 53 & 41 & 2173 & $\overline{0}$ & 0 & $\overline{0}$ \\
\hline $\bar{D}$ & $\overline{\mathbf{P}}$ & $480-002 D$ & 270 & 25 & 41) & 1025 & $\overline{0}$ & 0 & $\overline{0}$ \\
\hline $\bar{D}$ & $\overline{\mathbf{P}}$ & $480-003 \mathrm{D}$ & 576 & 54 & 41 & 2214 & $\overline{0}$ & 0 & $\overline{0}$ \\
\hline $\bar{D}$ & $\overline{\mathbf{P}}$ & $483-001 \mathrm{D}$ & 38000 & 3530 & 37 & 130610 & $\overline{0}$ & 0 & $\overline{0}$ \\
\hline D & $\mathbf{P}$ & $483-002 \mathrm{D}$ & 851 & 79 & 41 & 3239 & 0 & 0 & 0 \\
\hline $\bar{D}$ & $\overline{\mathbf{P}}$ & $483-003 \mathrm{D}$ & 1920 & 178 & 41 & 7298 & $\overline{0}$ & 0 & 0 \\
\hline D & $\mathbf{P}$ & 484-010D & 2400 & 223 & 61 & 1338 & 24 & 0 & 24 \\
\hline $\bar{D}$ & $\mathbf{P}$ & 484-011D & 192 & 18 & 6 & 108 & 0 & 0 & 0 \\
\hline D & $\mathbf{P}$ & $484-012 D$ & 800 & 74 & 6 & 444 & 0 & 0 & 0 \\
\hline $\bar{D}$ & $\overline{\mathbf{P}}$ & $484-002 \mathrm{D}$ & 648 & 60 & 15 & 900 & $\overline{1}$ & $\overline{0}$ & 1 \\
\hline $\bar{D}$ & $\overline{\mathbf{P}}$ & 484-003D & 550 & 51 & 14 & 714 & $\overline{0}$ & $\overline{0}$ & 0 \\
\hline $\bar{D}$ & $\overline{\mathbf{P}}$ & $484-004 D$ & 9200 & 855 & 13 & 11115 & 0 & $\overline{0}$ & $\overline{0}$ \\
\hline $\bar{D}$ & $\mathbf{P}$ & 484-005D & 240 & 22 & 13 & 286 & $\overline{0}$ & $\overline{0}$ & $\overline{0}$ \\
\hline $\bar{D}$ & $\overline{\mathbf{P}}$ & $484-007 D$ & 1600 & 149 & 7 & 1043 & $\overline{0}$ & $\overline{0}$ & $\overline{0}$ \\
\hline $\bar{D}$ & $\mathbf{P}$ & $484-000 \mathrm{D}$ & 250000 & 23226 & 37 & 859362 & 0 & 10 & 10 \\
\hline D & $\overline{\mathbf{P}}$ & $485-000 \mathrm{D}$ & 3826 & 355 & 37 & 13135 & 0 & ) & 0 \\
\hline $\bar{D}$ & $\overline{\mathbf{P}}$ & 494-006D & 168 & 16 & 14 & 224 & $\overline{0}$ & ) & \\
\hline
\end{tabular}


Table A-14.--Incipient fire data for 200F Area

\begin{tabular}{|c|c|c|c|c|c|c|c|c|c|}
\hline \multirow[b]{2}{*}{ Area } & \multirow[b]{2}{*}{ Type } & \multirow[b]{2}{*}{ ID } & \multirow[b]{2}{*}{ Area, $\mathrm{ft}^{2}$} & \multirow[b]{2}{*}{ Area, $\mathbf{m}^{2}$} & \multirow{2}{*}{$\begin{array}{c}\text { years in } \\
\text { service to } \\
' 94\end{array}$} & \multirow[b]{2}{*}{ yrs $^{*} \mathrm{~m}^{2}$} & \multicolumn{3}{|c|}{ Fire count } \\
\hline & & & & & & & $\begin{array}{c}\text { Original } \\
\text { count }\end{array}$ & '91 to '94 & $\begin{array}{l}\text { Sum of } \\
\text { all fires }\end{array}$ \\
\hline & & ब18. & 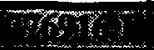 & 78 & & masts & $\pi$ & R. & 3 \\
\hline F & $\mathbf{P}$ & $200-000 \mathrm{~F}$ & 이 & 0 & 0 & 0 & 1 & 0 & 1 \\
\hline $\bar{F}$ & P & $211-000 \mathrm{~F}$ & 10000 & 929 & 37 & 34373 & 3 & 0 & 3 \\
\hline$F$ & $\mathbf{P}$ & $211-001 \mathrm{~F}$ & 6000 & 557 & 9 & 5013 & 0 & 1 & 1 \\
\hline $\mathbf{F}$ & $\mathbf{P}$ & $211-002 \mathrm{~F}$ & 740 & 69 & 40 & 2760 & $\overline{0}$ & 0 & $\overline{0}$ \\
\hline F & $\mathbf{P}$ & $211-003 \mathrm{~F}$ & 1900 & 177 & 40 & 7080 & 0 & 0 & 0 \\
\hline $\bar{F}$ & P & $211-004 \mathrm{~F}$ & 140 & 13 & 40 & 520 & 0 & 0 & 0 \\
\hline F & $P$ & $211-005 \mathrm{~F}$ & 288 & 27 & 40 & 1080 & 0 & 0 & 0 \\
\hline $\mathbf{F}$ & $\mathbf{P}$ & $211-007 \mathrm{~F}$ & 1800 & 167 & 7 & 1169 & 0 & 0 & 0 \\
\hline $\mathbf{F}$ & $\mathbf{P}$ & $217-000 \mathrm{~F}$ & 1024 & 95 & 40 & 3800 & 0 & 0 & 0 \\
\hline $\mathbf{F}$ & $\mathbf{P}$ & $221-000 \mathrm{~F}$ & 329720.1 & 30632 & 40 & 1225280 & 88 & 6 & 94 \\
\hline $\mathbf{F}$ & $\mathbf{P}$ & $221-001 \mathrm{~F}$ & 24067 & 2236 & 37 & 82732 & 1 & 1 & 2 \\
\hline $\mathbf{F}$ & $\mathbf{P}$ & $221-003 \mathrm{~F}$ & 4000 & 372 & 37 & 13764 & 0 & $\overline{0}$ & $\overline{0}$ \\
\hline $\mathbf{F}$ & P & $221-004 \mathrm{~F}$ & 192 & 18 & 37 & 666 & $\overline{0}$ & 0 & $\overline{0}$ \\
\hline $\bar{F}$ & $\mathbf{P}$ & $221-010 \mathrm{~F}$ & 1536 & 143 & 13 & 1859 & 0 & 0 & 0 \\
\hline $\mathbf{F}$ & $\mathbf{P}$ & $221-012 \mathrm{~F}$ & 10800 & 1003 & 7 & 7021 & $\overline{0}$ & 0 & 0 \\
\hline $\mathbf{F}$ & $\mathbf{P}$ & $221-013 \mathrm{~F}$ & 416 & 39 & 12 & 468 & $\overline{0}$ & $\overline{0}$ & $\overline{0}$ \\
\hline $\mathbf{F}$ & $\mathbf{P}$ & $221-015 \mathrm{~F}$ & 1536 & 143 & 9 & 1287 & $\overline{0}$ & 0 & 0 \\
\hline $\mathbf{F}$ & $\mathbf{P}$ & $221-016 \mathrm{~F}$ & 1008 & 94 & 9 & 846 & 0 & 0 & 0 \\
\hline $\mathbf{F}$ & P & $221-021 \mathrm{~F}$ & 20000 & 1858 & 7 & 13006 & 0 & 0 & 0 \\
\hline $\mathbf{F}$ & $\mathbf{P}$ & $221-022 \mathrm{~F}$ & 20000 & 1858 & 7 & 13006 & $\overline{0}$ & 0 & 0 \\
\hline $\mathbf{F}$ & $\mathbf{P}$ & $221-025 \mathrm{~F}$ & 20880 & 1940 & 9 & 17460 & $\overline{0}$ & 1 & 1 \\
\hline $\mathbf{F}$ & $\mathbf{P}$ & $221-026 \mathrm{~F}$ & 800 & 74 & 7 & 518 & 0 & 0 & 0 \\
\hline $\mathbf{F}$ & $\mathbf{P}$ & $221-027 F$ & 1000 & 93 & 6 & 558 & $\overline{0}$ & 0 & 0 \\
\hline $\mathbf{F}$ & $\mathbf{P}$ & $222-000 \mathrm{~F}$ & 5989 & 556 & 37 & 20572 & 1 & 0 & 1 \\
\hline $\mathbf{F}$ & $\mathbf{P}$ & $223-000 \mathrm{~F}$ & 390 & 36 & 32 & 1152 & $\overline{0}$ & 0 & 0 \\
\hline $\mathbf{F}$ & $\mathbf{P}$ & $232-000 \mathrm{~F}$ & 16440 & 1527 & 40 & 61080 & 1 & 0 & 1 \\
\hline F & $\mathbf{P}$ & $235-000 \mathrm{~F}$ & 47080 & 4374 & 37 & 161838 & $\overline{5}$ & 0 & 5 \\
\hline $\bar{F}$ & $\overline{\mathbf{P}}$ & $235-001 \mathrm{~F}$ & 950 & 88 & 16 & 1408 & $\overline{0}$ & 0 & 0 \\
\hline $\mathbf{F}$ & $\bar{P}$ & $235-002 \mathrm{~F}$ & 476 & 44 & 9 & 396 & $\overline{0}$ & 0 & 0 \\
\hline $\mathbf{F}$ & $\mathbf{P}$ & $235-006 \mathrm{~F}$ & 288 & 27 & 9 & 243 & $\overline{0}$ & 0 & 0 \\
\hline $\bar{F}$ & $\mathbf{P}$ & $235-009 \mathrm{~F}$ & 288 & 27 & 9 & 243 & $\overline{0}$ & $\overline{0}$ & 0 \\
\hline$\overline{\mathbf{F}}$ & $\mathbf{P}$ & $235-011 \mathrm{~F}$ & 1792 & 166 & 5 & 830 & $\overline{0}$ & 0 & 0 \\
\hline $\mathbf{F}$ & $\mathbf{P}$ & $240-000 \mathrm{~F}$ & 3072 & 285 & 43 & 12255 & 0 & 0 & 0 \\
\hline $\mathbf{F}$ & $\overline{\mathbf{P}}$ & $241-000 \mathrm{~F}$ & 714 & 66 & 9 & 594 & 1 & 0 & 1 \\
\hline$F$ & $\mathbf{P}$ & $241-017 \mathrm{~F}$ & 1078 & 100 & 14 & 1400 & 0 & 0 & 0 \\
\hline $\mathbf{F}$ & $\mathbf{P}$ & $241-018 \mathrm{~F}$ & 1200 & 111 & 14 & 1554 & 0 & 0 & 0 \\
\hline $\mathbf{F}$ & $\mathbf{P}$ & $241-024 \mathrm{~F}$ & 1500 & 139 & 14 & 1946 & 1 & 0 & 1 \\
\hline $\mathbf{F}$ & $\overline{\mathbf{P}}$ & $241-028 \mathrm{~F}$ & 1000 & 93 & 12 & 1116 & $\overline{0}$ & 1 & 1 \\
\hline $\mathbf{F}$ & $\bar{P}$ & $241-051 \mathrm{~F}$ & 36 & 3 & 14 & 42 & 0 & 0 & 0 \\
\hline $\mathbf{F}$ & $\mathbf{P}$ & $241-053 \mathrm{~F}$ & 300 & 28 & 14 & 392 & 0 & 0 & 0 \\
\hline $\mathbf{F}$ & $\mathbf{P}$ & $241-058 \mathrm{~F}$ & 2400 & 223 & 14 & 3122 & 0 & 0 & 0 \\
\hline $\mathbf{F}$ & P & $241-062 \mathrm{~F}$ & 390 & 36 & 10 & 360 & 0 & 0 & 0 \\
\hline $\mathbf{F}$ & $\mathbf{P}$ & $241-064 \mathrm{~F}$ & 486 & 45 & 11 & 495 & 0 & 1 & 1 \\
\hline $\mathbf{F}$ & $\mathbf{P}$ & $241-065 \mathrm{~F}$ & 360 & 33 & 11 & 363 & 0 & 0 & 0 \\
\hline $\mathbf{F}$ & $\mathbf{P}$ & $241-072 \mathrm{~F}$ & 360 & 33 & 11 & 363 & 0 & 0 & 0 \\
\hline $\bar{F}$ & $\mathbf{P}$ & $241-080 \mathrm{~F}$ & 600 & 56 & 11 & 616 & 0 & 0 & 0 \\
\hline
\end{tabular}


Table A-14.--Incipient fire data for 200F Area

\begin{tabular}{|c|c|c|c|c|c|c|c|c|c|}
\hline \multirow[b]{2}{*}{ Area } & \multirow[b]{2}{*}{ Type } & \multirow[b]{2}{*}{ ID } & \multirow[b]{2}{*}{ Area, $\mathbf{f t}^{2}$} & \multirow[b]{2}{*}{ Area, $\mathbf{m}^{2}$} & \multirow{2}{*}{\begin{tabular}{|c|} 
years in \\
service to \\
94
\end{tabular}} & \multirow[b]{2}{*}{$\mathrm{yrs}^{*} \mathrm{rn}^{2}$} & \multicolumn{3}{|c|}{ Fire count } \\
\hline & & & & & & & $\begin{array}{c}\text { Original } \\
\text { count }\end{array}$ & $\mid$ '91 to $94 \mid$ & $\begin{array}{l}\text { Sum of } \\
\text { all fires }\end{array}$ \\
\hline$F$ & $\mathbf{P}$ & $241-084 \mathrm{~F}$ & 400 & 37 & 8 & 296 & 0 & 이 & 0 \\
\hline $\mathbf{F}$ & $\mathrm{P}$ & $241-091 \mathrm{~F}$ & 2400 & 223 & 8 & 1784 & 0 & 0 & 0 \\
\hline $\mathrm{F}$ & $\mathrm{P}$ & 241-093F & 608 & 56 & 8 & \begin{tabular}{l|l|}
448 \\
\end{tabular} & 0 & 0 & 0 \\
\hline $\mathbf{F}$ & P & $241-096 \mathrm{~F}$ & 144 & 13 & 7 & 91 & 0 & 0 & 0 \\
\hline $\mathrm{F}$ & $\mathbf{P}$ & $241-100 \mathrm{~F}$ & 1792 & 166 & 6 & 996 & 0 & 0 & 0 \\
\hline F & $\mathrm{P}$ & $242-001 \mathrm{~F}$ & 913 & 85 & 12 & 1020 & 0 & 0 & 0 \\
\hline$F$ & $P$ & $246-000 \mathrm{~F}$ & 13660 & 1269 & 17 & 21573 & 0 & of & 0 \\
\hline $\mathbf{F}$ & $\mathrm{P}$ & $246-001 F$ & 240 & 22 & 8 & 176 & 0 & of & 0 \\
\hline$\overline{\mathbf{F}}$ & $\mathrm{P}$ & $246-002 F$ & 1792 & 166 & 6 & 996 & 이 & 0 & 0 \\
\hline $\mathrm{F}$ & $\mathrm{P}$ & $247-000 \mathrm{~F}$ & 140000 & 13006 & 81 & 104048 & 4 & 1 & 5 \\
\hline $\mathbf{F}$ & $\mathrm{P}$ & $247-003 \mathrm{~F}$ & 1836 & 171 & 6 & 1026 & 0 & 0 & 0 \\
\hline $\mathbf{F}$ & P & $247.004 \mathrm{~F}$ & 180 & 17 & 6 & 102 & 0 & of & 0 \\
\hline $\mathbf{F}$ & $P$ & $247-007 \mathrm{~F}$ & 600 & 56 & 6 & 336 & 0] & of & 0 \\
\hline$F$ & $\mathbf{P}$ & $247-008 \mathrm{~F}$ & 276 & 26 & 61 & 156 & 이 & 0 & 0 \\
\hline $\mathbf{F}$ & $\mathbf{P}$ & $247-009 \mathrm{~F}$ & 240 & 22 & 6 & 132 & 이 & o! & 0 \\
\hline $\mathbf{F}$ & $\mathbf{P}$ & $247-011 \mathrm{~F}$ & 180 & 17 & 6 & 102 & 0 & 0| & 0 \\
\hline $\mathbf{F}$ & $\mathbf{P}$ & $247-012 \mathrm{~F}$ & 3795 & 353 & 6 & 2118 & 0 & 0 & 0 \\
\hline$F$ & $\mathbf{P}$ & $247-041 \mathrm{~F}$ & 1200 & 111 & 6 & 666 & 0 & 0 & 0 \\
\hline $\bar{F}$ & $\mathbf{P}$ & $251-000 \mathrm{~F}$ & 1923 & 179 & 37 & 6623 & 11 & 0 & 1 \\
\hline $\bar{F}$ & $\mathrm{P}$ & $254-002 \mathrm{~F}$ & 520 & 48 & 17 & 816 & 0 & 0 & 0 \\
\hline $\bar{F}$ & $\mathbf{P}$ & $254-005 \mathrm{~F}$ & 816 & 76 & 14 & 1064 & 0 & 0 & 0 \\
\hline $\mathrm{F}$ & $\mathbf{P}$ & $260-001 \mathrm{~F}$ & 80 & 7 & 40 & 280 & 0 & 0 & 0 \\
\hline $\mathbf{F}$ & $\mathbf{P}$ & $280-001 \mathrm{~F}$ & 944 & 88 & 40 & 3520 & of & 0 & 0 \\
\hline $\bar{F}$ & $\mathbf{P}$ & $280-002 \mathrm{~F}$ & 400 & 37 & 40 & 1480 & 0 & 0 & 0 \\
\hline $\bar{F}$ & $P$ & $281-004 \mathrm{~F}$ & 250 & 23 & 40 & 920 & 0 & of & 0 \\
\hline $\bar{F}$ & $\bar{P}$ & $281-006 \mathrm{~F}$ & 250 & 23 & 40 & 920 & 0 & of & $\overline{0}$ \\
\hline $\mathbf{F}$ & $\mathbf{P}$ & $281-012 \mathrm{~F}$ & 112 & 10 & 6 & 60 & 0 & 0 & 0 \\
\hline $\mathbf{F}$ & $\bar{P}$ & $281-019 \mathrm{~F}$ & 144 & 13 & 6 & 78 & 0 & 0 & 0 \\
\hline $\mathbf{F}$ & $\mathbf{P}$ & $281-020 \mathrm{~F}$ & 144 & 13 & 6 & 78 & 0 & 0 & 0 \\
\hline $\mathbf{F}$ & $\mathbf{P}$ & $281-021 \mathrm{~F}$ & 144 & 13 & 6 & 78 & 0 & 0 & 0 \\
\hline $\mathbf{F}$ & $\mathbf{P}$ & $281-022 \mathrm{~F}$ & 144 & 13 & 6 & 78 & 0 & 0 & 0 \\
\hline$F$ & $\mathbf{P}$ & $281-023 F$ & 144 & 13 & 6 & 78 & 0 & 0 & 0 \\
\hline $\mathrm{F}$ & $\mathrm{P}$ & $281-024 \mathrm{~F}$ & 144 & 13 & 6 & 78 & 0 & 0 & 0 \\
\hline$F$ & $\mathbf{P}$ & $281-025 \mathrm{~F}$ & 144 & 13 & 6 & 78 & 0 & 0 & 0 \\
\hline $\bar{F}$ & $\mathbf{P}$ & $282-000 \mathrm{~F}$ & 210 & 20 & 38 & 760 & 0 & of & 0 \\
\hline$F$ & $\mathbf{P}$ & $284-000 \mathrm{~F}$ & 30000 & 2787 & 40 & 111480 & 4 & of & 4 \\
\hline $\mathbf{F}$ & $\mathbf{P}$ & $284-002 \mathrm{~F}$ & 500 & 46 & 21 & 966 & 의 & of & 0 \\
\hline $\mathbf{F}$ & $\mathbf{P}$ & 284-003F & 240 & 22 & 14 & 308 & 의 & 요 & 0 \\
\hline $\mathbf{F}$ & $\mathbf{P}$ & $284-004 \mathrm{~F}$ & 840 & 78 & 14 & 1092 & 요 & 0 & 0 \\
\hline $\mathbf{F}$ & $\mathbf{P}$ & $284-005 \mathrm{~F}$ & 240 & 22 & 14 & 308 & 요 & 0 & 0 \\
\hline$F$ & $\mathbf{P}$ & $284-008 \mathrm{~F}$ & 2826 & 263 & 9 & 2367 & 0 & 0 & $\overline{0}$ \\
\hline $\mathbf{F}$ & $\mathbf{P}$ & $284-009 \mathrm{~F}$ & 3600 & 334 & 6 & 2004 & 0 & 0 & 0 \\
\hline$F$ & $\overline{\mathbf{P}}$ & $285-001 \mathrm{~F}$ & 60 & 6 & 30 & 180 & o) & 0 & $\overline{0}$ \\
\hline $\mathbf{F}$ & $\mathbf{P}$ & $292.000 \mathrm{~F}$ & 12482 & 1160 & 37 & 42920 & 5 & 2 & 7 \\
\hline F & $\mathbf{P}$ & $292-001 \mathrm{~F}$ & 613 & 57 & 40 & 2280 & 1 & 0 & 1 \\
\hline $\bar{F}$ & $\mathbf{P}$ & $292-002 \mathrm{~F}$ & 3470 & 322 & 5 & 1610 & 0) & 0 & 0 \\
\hline $\mathbf{F}$ & $\mathbf{P}$ & 294-001F & 240 & 22 & 8 & 176 & 0 & 0 & 0 \\
\hline
\end{tabular}


Table A-14.--Incipient fire data for 200F Area

\begin{tabular}{|c|c|c|c|c|c|c|c|c|c|}
\hline & & & & & years in & & & Fire count & \\
\hline Area & Type & ID & Area, $\mathrm{ft}^{2}$ & Area, $\mathbf{m}^{2}$ & $\begin{array}{c}\text { service to } \\
94\end{array}$ & yrs* $m^{2}$ & $\begin{array}{c}\text { Origina } \\
\text { count }\end{array}$ & '91 to '94 & $\begin{array}{l}\text { Sum of } \\
\text { all fires }\end{array}$ \\
\hline
\end{tabular}


Table A-15.--Incipient fire data for $200 \mathrm{H}$ Area

\begin{tabular}{|c|c|c|c|c|c|c|c|c|c|}
\hline \multirow[b]{2}{*}{ Area } & \multirow[b]{2}{*}{ Type } & \multirow[b]{2}{*}{ ID } & \multirow[b]{2}{*}{ Area, $\mathrm{ft}^{2}$} & \multirow[b]{2}{*}{ Area, $\mathbf{m}^{2}$} & \multirow{2}{*}{$\begin{array}{c}\text { years in } \\
\text { service to } \\
.94\end{array}$} & \multirow[b]{2}{*}{$y r s * a^{2}$} & \multicolumn{3}{|c|}{ Fire count } \\
\hline & & & & & & & $\begin{array}{c}\text { Original } \\
\text { count }\end{array}$ & '91 to '94] & $\begin{array}{l}\text { Sum of } \\
\text { all fires }\end{array}$ \\
\hline & & DN & Drs? & 6ro & & Mrag 7 & s. & 5 & 7 \\
\hline $\mathrm{H}$ & $P$ & $200-000 \mathrm{H}$ & 0 & 0 & 0 & 이 & 1 & 이 & 1 \\
\hline $\mathrm{H}$ & $\mathbf{P}$ & $211.000 \mathrm{H}$ & 10000 & 929 & 37 & 34.373 & 0 & of & 0 \\
\hline $\mathrm{H}$ & $\mathrm{P}$ & $211-002 \mathrm{H}$ & 1500 & 139 & 37 & 5143 & 0 & 0 & 0 \\
\hline $\mathrm{H}$ & $\mathbf{P}$ & $211-004 \mathrm{H}$ & 141 & 13 & 39 & 507 & 0 & 0 & 0 \\
\hline $\mathrm{H}$ & $\mathbf{P}$ & $211-007 \mathrm{H}$ & 10000 & 929 & 37 & 34.373 & 4 & 0 & 4 \\
\hline $\mathrm{H}$ & $\mathbf{P}$ & $211-008 \mathrm{H}$ & 3000 & 279 & 8 & 232 & 0 & 0 & 0 \\
\hline $\mathrm{H}$ & $\mathbf{P}$ & $211-009 \mathrm{H}$ & 300 & 28 & 8 & 224 & 0 & 0 & 0 \\
\hline $\mathrm{H}$ & $\mathbf{P}$ & $211-010 \mathrm{H}$ & 300 & 28 & 8 & 224 & 0 & 0 & 0 \\
\hline $\mathrm{H}$ & $\mathbf{P}$ & $217-000 \mathrm{H}$ & 901 & 84 & 36 & 3024 & 0 & 0 & 0 \\
\hline $\mathbf{H}$ & $\mathbf{P}$ & $218-000 \mathrm{H}$ & 2800 & 260 & 9 & 1340 & 0 & 1 & 1 \\
\hline $\mathrm{H}$ & $\mathbf{P}$ & $221-000 \mathrm{H}$ & 329600 & 30621 & 40 & 1224840 & 27 & 6 & 33 \\
\hline $\mathrm{H}$ & $\mathbf{P}$ & $221-004 \mathrm{H}$ & 1500 & 139 & 27 & 3753 & 0 & 0 & 0 \\
\hline $\mathrm{H}$ & $\mathbf{P}$ & $221-006 \mathrm{H}$ & 240 & 22 & 8 & 176 & 0 & 0 & 0 \\
\hline $\mathrm{H}$ & $\mathbf{P}$ & $221-012 \mathrm{H}$ & 160 & 15 & 9 & 135 & 0 & 이 & 0 \\
\hline $\mathrm{H}$ & $\mathbf{P}$ & $221-013 \mathrm{H}$ & 600 & 56 & 9 & 504 & 0 & 0 & 0 \\
\hline $\mathrm{H}$ & $\mathrm{P}$ & $221-014 \mathrm{H}$ & 600 & 56 & 9 & 504 & 0 & of & 0 \\
\hline $\mathrm{H}$ & $\mathbf{P}$ & $225-001 \mathrm{H}$ & 896 & 83 & 9 & 747 & 이 & 요 & 0 \\
\hline $\mathrm{H}$ & $\mathbf{P}$ & $225-002 \mathrm{H}$ & 1792 & 166 & 9 & 1494 & 0 & 임 & 0 \\
\hline $\mathrm{H}$ & $\mathbf{P}$ & $225-003 \mathrm{H}$ & 1792 & 166 & 9 & 1494 & 0 & 0 & 0 \\
\hline $\mathrm{H}$ & $\mathbf{P}$ & $225-007 \mathrm{H}$ & 1792 & 166 & 6 & 996 & 0 & 0 & 0 \\
\hline $\mathrm{H}$ & $\mathbf{P}$ & $225-008 \mathrm{H}$ & 1792 & 166 & 5 & 830 & 0 & 0 & 0 \\
\hline $\mathrm{H}$ & $P$ & $225-009 \mathrm{H}$ & 840 & 78 & 13 & 1014 & 0 & 0 & 0 \\
\hline $\mathrm{H}$ & $\mathbf{P}$ & $225-010 \mathrm{H}$ & 960 & 89 & 13 & 1157 & 요 & 요 & 0 \\
\hline $\mathrm{H}$ & $\mathbf{P}$ & $225-011 \mathrm{H}$ & 960 & 89 & 13 & 1157 & 이 & 0 & 0 \\
\hline $\mathrm{H}$ & $\mathbf{P}$ & $225-012 \mathrm{H}$ & 960 & 89 & 13 & 1157 & of & 0 & 0 \\
\hline $\mathrm{H}$ & $\mathrm{P}$ & $225-013 \mathrm{H}$ & 960 & 89 & 13 & 1157 & 0 & 0 & 0 \\
\hline $\mathrm{H}$ & $\mathbf{P}$ & $225-014 \mathrm{H}$ & 960 & 89 & 13 & 1157 & 0 & of & 0 \\
\hline $\mathrm{H}$ & P & $225-015 \mathrm{H}$ & 960 & 89 & 13 & 1157 & of & 이 & 0 \\
\hline $\mathrm{H}$ & $\mathbf{P}$ & $225-017 \mathrm{H}$ & 960 & 89 & 13 & 1157 & 0 & 0 & 0 \\
\hline $\mathrm{H}$ & $\mathbf{P}$ & $225-018 \mathrm{H}$ & 504 & 47 & 13 & 611 & 0 & 0 & 0 \\
\hline $\mathrm{H}$ & $P$ & $230-000 \mathrm{H}$ & 7165 & 666 & 11 & 7326 & 1 & 0 & 1 \\
\hline $\mathrm{H}$ & $P$ & $232-000 \mathrm{H}$ & 55220 & 5130 & 39 & 200070 & 9 & 0 & 9 \\
\hline $\mathrm{H}$ & $\mathbf{P}$ & $232-001 \mathrm{H}$ & 11000 & 1022 & 37 & 37814 & 0 & 0 & 0 \\
\hline $\mathrm{H}$ & $P$ & $233-000 \mathrm{H}$ & 33000 & 3066 & 11 & 33726 & 0 & 0 & 0 \\
\hline $\mathrm{H}$ & $\mathrm{P}$ & $233-004 \mathrm{H}$ & 1792 & 166 & 3 & 498 & 0 & 0 & 0 \\
\hline $\mathrm{H}$ & $\mathbf{P}$ & $233-006 \mathrm{H}$ & 1792 & 166 & 6 & 996 & 0 & 0 & 0 \\
\hline $\mathrm{H}$ & $\mathbf{P}$ & $233-007 \mathrm{H}$ & 1792 & 166 & 6 & 996 & 0 & 0 & 0 \\
\hline $\mathrm{H}$ & $\mathbf{P}$ & $233-008 \mathrm{H}$ & 1792 & 166 & 6 & 996 & 0 & 0 & 0 \\
\hline $\mathrm{H}$ & $\mathbf{P}$ & $233-009 \mathrm{H}$ & 1792 & 166 & 5 & 830 & 0 & 0 & 0 \\
\hline $\mathrm{H}$ & $\mathrm{P}$ & $233-010 \mathrm{H}$ & 1792 & 166 & 5 & 830 & 0 & 0 & 0 \\
\hline $\mathrm{H}$ & P & 233-011H & 432 & 40 & 5 & 200 & 0 & 0 & 0 \\
\hline $\mathrm{H}$ & P & $233-012 \mathrm{H}$ & 288 & 27 & 5 & 135 & 0 & 0 & 0 \\
\hline $\mathrm{H}$ & $P$ & 234-000H & 57383 & 5331 & 36 & 151916 & 5 & 1 & 6 \\
\hline $\mathrm{H}$ & P & $234-002 \mathrm{H}$ & 672 & 62 & 9 & 558 & 0 & 0 & 0 \\
\hline $\mathrm{H}$ & $\mathbf{P}$ & $235-000 \mathrm{H}$ & 12000 & 1115 & 10 & 11150 & 0 & 0 & 0 \\
\hline $\mathrm{H}$ & $\mathrm{P}$ & $235-001 \mathrm{H}$ & 720 & 67 & 6) & 402 & 0 & 0 & $\underline{0}$ \\
\hline
\end{tabular}


Table A-15.--Incipient fire data for $200 \mathrm{H}$ Area

\begin{tabular}{|c|c|c|c|c|c|c|c|c|c|}
\hline \multirow[b]{2}{*}{ Area } & \multirow[b]{2}{*}{ Type } & \multirow[b]{2}{*}{ ID } & \multirow[b]{2}{*}{ Area, $\mathbf{f t}^{2}$} & \multirow[b]{2}{*}{ Area, $\mathrm{m}^{2}$} & \multirow{2}{*}{$\begin{array}{c}\text { years in } \\
\text { service to } \\
' 94\end{array}$} & \multirow[b]{2}{*}{$\operatorname{yrs}^{*} \mathrm{~m}^{2}$} & \multicolumn{3}{|c|}{ Fire count } \\
\hline & & & & & & & $\begin{array}{c}\text { Origina } \\
\text { count }\end{array}$ & '91 to ' $94 \mid$ & $\begin{array}{l}\text { Sum of } \\
\text { all fires }\end{array}$ \\
\hline $\mathrm{H}$ & $\mathbf{P}$ & $235-002 \mathrm{H}$ & 720 & 67 & 6 & 402 & 0 & 이 & 0 \\
\hline $\mathrm{H}$ & $\mathbf{P}$ & $235-007 \mathrm{H}$ & 510 & 47 & 6 & 282 & 0 & 0 & 0 \\
\hline $\mathrm{H}$ & $\mathbf{P}$ & $235-009 \mathrm{H}$ & 1792 & 166 & 6 & 996 & 0 & 0 & 0 \\
\hline $\mathbf{H}$ & $\mathbf{P}$ & $235-010 \mathrm{H}$ & 1792 & 166 & 6 & 996 & 0 & 0 & 0 \\
\hline $\mathrm{H}$ & P & $236-000 \mathrm{H}$ & 1270 & 118 & 24 & 2832 & of & 0 & 0 \\
\hline $\mathrm{H}$ & P & $237-000 \mathrm{H}$ & 5675 & 527 & 24 & 12648 & 0 & 0 & 0 \\
\hline $\mathrm{H}$ & $\mathbf{P}$ & $238-000 \mathrm{H}$ & 17175 & 1596 & 25 & 39900 & 0 & 01 & 0 \\
\hline$\overline{\mathrm{H}}$ & $\bar{P}$ & $238-001 \mathrm{H}$ & 640 & 59 & 25 & 1475 & 0 & 0 & $\overline{0}$ \\
\hline $\mathrm{H}$ & $\bar{P}$ & $241-000 \mathrm{H}$ & 1725 & 160 & 14 & 2240 & 0 & 11 & 1 \\
\hline $\mathrm{H}$ & P & $241-010 \mathrm{H}$ & 9713 & 902 & 14 & 12628 & 0 & 0 & 0 \\
\hline $\mathrm{H}$ & $P$ & $241-013 \mathrm{H}$ & 640 & 59 & 15 & 885 & of & 0 & 0 \\
\hline $\mathrm{H}$ & P & 241-017H & 363 & 34 & 18 & 612 & 0 & 0 & 0 \\
\hline $\mathrm{H}$ & $\mathbf{P}$ & $\longdiv { 2 4 1 - 0 2 3 \mathrm { H } }$ & 720 & 67 & 14 & 938 & 0 & 0 & $\overline{0}$ \\
\hline $\mathbf{H}$ & $\mathbf{P}$ & $241-024 \mathrm{H}$ & 1536 & 143 & 17 & 2431 & 1 & 0 & 1 \\
\hline $\mathrm{H}$ & $\mathbf{P}$ & $241-025 \mathrm{H}$ & 120 & 11 & 14 & 154 & 0 & 0 & 0 \\
\hline $\mathrm{H}$ & $\mathbf{P}$ & $241-026 \mathrm{H}$ & 36 & 31 & 14 & 42 & 0 & 0 & 0 \\
\hline $\mathrm{H}$ & P & $241-028 \mathrm{H}$ & 100 & 9 & 12 & 108 & 0 & 0 & 0 \\
\hline $\mathrm{H}$ & $\mathbf{P}$ & $241-049 \mathrm{H}$ & 100 & 9 & 12 & 108 & 0 & 이 & 0 \\
\hline $\mathbf{H}$ & $\mathbf{P}$ & $241-052 \mathrm{H}$ & 288 & 27 & 12 & 324 & 0 & 0 & 0 \\
\hline $\mathbf{H}$ & $\mathbf{P}$ & $241-055 \mathrm{H}$ & 1875 & 174 & 12 & 2088 & 1 & 0 & 1 \\
\hline $\mathrm{H}$ & $\mathrm{P}$ & $241-057 \mathrm{H}$ & 800 & 74 & 12 & 888 & 0 & 0 & 0 \\
\hline $\mathrm{H}$ & $\mathrm{P}$ & $241-058 \mathrm{H}$ & 2508 & 233 & 12 & 2796 & 1 & 0 & 1 \\
\hline $\mathrm{H}$ & $\mathbf{P}$ & $241-062 \mathrm{H}$ & 390 & 36 & 10 & 360 & 0 & 0 & 0 \\
\hline $\mathrm{H}$ & $\mathbf{P}$ & $241-064 \mathrm{H}$ & 480 & 45 & 10 & 450 & 0 & 0 & 0 \\
\hline $\mathrm{H}$ & $\mathbf{P}$ & $241-065 \mathrm{H}$ & 360 & 33 & 10 & 330 & 0 & 0 & $\overline{0}$ \\
\hline $\mathrm{H}$ & $\mathrm{P}$ & $241-068 \mathrm{H}$ & 580 & 54 & 10 & 540 & 0 & 0 & 0 \\
\hline $\mathrm{H}$ & $\bar{P}$ & $241-069 \mathrm{H}$ & 109 & 10 & 10 & 100 & 0 & 0 & $\overline{0}$ \\
\hline $\bar{H}$ & $\mathbf{P}$ & $241-070 \mathrm{H}$ & 540 & 50 & 10 & 500 & 0 & 0 & $\overline{0}$ \\
\hline $\mathrm{H}$ & $\mathbf{P}$ & $241-071 \mathrm{H}$ & 288 & 27 & 10 & 270 & 0 & 0 & $\overline{0}$ \\
\hline $\mathrm{H}$ & $\mathbf{P}$ & $241-072 \mathrm{H}$ & 360 & 33 & 14 & 462 & 0 & 0 & 0 \\
\hline $\bar{H}$ & $\mathrm{P}$. & $241-080 \mathrm{H}$ & 600 & 56 & 12 & 672 & 0 & 0 & 0 \\
\hline$\vec{H}$ & $\mathbf{P}$ & $241-081 \mathrm{H}$ & 16500 & 1533 & 10 & 15330 & 0 & 0 & 0 \\
\hline $\bar{H}$ & $\mathbf{P}$ & $241-082 \mathrm{H}$ & 1800 & 167 & 1 & 167 & of & 1 & 1 \\
\hline H & P & $241-084 \mathrm{H}$ & 6240 & 580 & 10 & 5800 & 0 & 0| & 0 \\
\hline $\mathrm{H}$ & $\mathbf{P}$ & $241-085 H$ & 400 & 37 & 8 & 296 & 0 & 요 & 0 \\
\hline $\mathbf{H}$ & $\mathbf{P}$ & $241-087 \mathrm{H}$ & 100 & 9 & 8 & 72 & 0 & o & 0 \\
\hline $\mathrm{H}$ & $P$ & $241-088 \mathrm{H}$ & 100 & 9 & 8 & 72 & 0 & 0 & $\overline{0}$ \\
\hline $\mathrm{H}$ & $\mathbf{P}$ & $241-089 \mathrm{H}$ & 1800 & 167 & 8 & 1336 & 0 & of & 0 \\
\hline $\mathbf{H}$ & $\mathbf{P}$ & $241-090 \mathrm{H}$ & 960 & 89 & 8 & 712 & 이 & 이 & 0 \\
\hline $\mathbf{H}$ & $\mathbf{P}$ & $241-092 \mathrm{H}$ & 1200 & 111 & 8 & 888 & 0 & 이 & 0 \\
\hline $\mathrm{H}$ & P & $241-095 \mathrm{H}$ & 672 & 62 & 8 & 496 & 0 & 이 & 0 \\
\hline $\mathrm{H}$ & $\mathbf{P}$ & $241-098 \mathrm{H}$ & 144 & 13 & 7 & 91 & 0 & of & 0 \\
\hline $\mathbf{H}$ & $P$ & $241-099 \mathrm{H}$ & 144 & 13 & 7 & 91 & 0 & 0 & $\overline{0}$ \\
\hline $\mathrm{H}$ & P & $241-105 \mathrm{H}$ & 750 & 70 & 6 & 420 & 0 & 0 & $\overline{0}$ \\
\hline $\bar{H}$ & $\bar{P}$ & $241-109 \mathrm{H}$ & 360 & 33 & 6 & 198 & 0 & 0 & 0 \\
\hline$\overline{\mathbf{H}}$ & $\bar{P}$ & $241-110 \mathrm{H}$ & 480 & 45 & 6 & 270 & 0 & 0 & 0 \\
\hline $\mathrm{H}$ & P & $241-119 \mathrm{H}$ & 1792 & 166 & 6 & 996 & 0 & 0 & 0 \\
\hline
\end{tabular}


Table A-15.--Incipient fire data for $200 \mathrm{H}$ Area

\begin{tabular}{|c|c|c|c|c|c|c|c|c|c|}
\hline \multirow[b]{2}{*}{ Area } & \multirow[b]{2}{*}{ Type } & \multirow[b]{2}{*}{ ID } & \multirow[b]{2}{*}{ Area, $\mathbf{f t}^{2}$} & \multirow[b]{2}{*}{ Area, $\mathrm{m}^{2}$} & \multirow{2}{*}{$\left|\begin{array}{c}\text { years in } \\
\text { service to } \\
\cdot 94\end{array}\right|$} & \multirow[b]{2}{*}{$\mathrm{yrs}^{*} \mathrm{~m}^{2}$} & \multicolumn{3}{|c|}{ Fire count } \\
\hline & & & & & & & $\begin{array}{c}\text { Original } \\
\text { count }\end{array}$ & '91 to '94 & $\begin{array}{l}\text { Sum of } \\
\text { all fires }\end{array}$ \\
\hline $\mathrm{H}$ & $\mathbf{P}$ & $241-120 \mathrm{H}$ & 1792 & 166 & 6 & 996 & 이 & 이 & 0 \\
\hline $\mathrm{H}$ & $\mathbf{P}$ & $241-121 \mathrm{H}$ & 1792 & 166 & 6 & 996 & 0 & 0 & 0 \\
\hline $\mathrm{H}$ & $\mathbf{P}$ & $241-122 \mathrm{H}$ & 504 & 47 & 6 & 282 & 0 & 0 & 0 \\
\hline $\mathrm{H}$ & $P$ & $241 \mathrm{~A}-38 \mathrm{H}$ & 375 & 35 & 9 & 315 & 0 & of & 0 \\
\hline $\mathrm{H}$ & $\mathbf{P}$ & $241 \mathrm{~A}-39 \mathrm{H}$ & 375 & 35 & 9 & 315 & 0 & 의 & 0 \\
\hline $\mathrm{H}$ & $\mathrm{P}$ & $241 \mathrm{~A}-40 \mathrm{H}$ & 375 & 35 & 9 & 315 & 0 & 0 & 0 \\
\hline$\overline{\mathrm{H}}$ & $\mathbf{P}$ & $241 \mathrm{~A}-41 \mathrm{H}$ & 375 & 35 & 9 & 315 & 0 & of & 0 \\
\hline $\mathbf{H}$ & $\mathbf{P}$ & $241 \mathrm{~A}-42 \mathrm{H}$ & 375 & 35 & 9 & 315 & 0 & 0 & 0 \\
\hline $\mathrm{H}$ & $\mathrm{P}$ & $241 \mathrm{~A}-43 \mathrm{H}$ & 375 & 35 & 9 & 315 & 0 & 0 & 0 \\
\hline $\mathbf{H}$ & $\mathbf{P}$ & $242-001 \mathrm{H}$ & 720 & 67 & 32 & 2144 & 1 & 0 & 1 \\
\hline $\mathrm{H}$ & $P$ & $242-016 \mathrm{H}$ & 300 & 28 & 12 & 336 & 0 & 0 & 0 \\
\hline $\mathrm{H}$ & $\mathbf{P}$ & $242-019 \mathrm{H}$ & 560 & 52 & 10 & 520 & 0 & 0 & 0 \\
\hline $\mathrm{H}$ & $\bar{P}$ & $242-023 \mathrm{H}$ & 200 & 19 & 10 & 190 & 0 & 0 & 0 \\
\hline $\mathrm{H}$ & $\overline{\mathbf{P}}$ & $242-024 \mathrm{H}$ & 3000 & 279 & 8 & 2232 & 0 & of & 0 \\
\hline $\mathbf{H}$ & $\mathbf{P}$ & $242.025 \mathrm{H}$ & 1674 & 156 & 9 & 1404 & 0 & 1 & 1 \\
\hline$\overline{\mathrm{H}}$ & $\mathbf{P}$ & $244-000 \mathrm{H}$ & 20785 & 1931 & 32 & 61792 & 1 & 0 & 1 \\
\hline $\mathbf{H}$ & $\mathbf{P}$ & $244-001 \mathrm{H}$ & 1920 & 178 & 25 & 4450 & 0 & 0 & 0 \\
\hline$\overline{\mathrm{H}}$ & $\overline{\mathbf{P}}$ & $244-002 \mathrm{H}$ & 1440 & 134 & 17 & 2278 & 0 & 0 & $\overline{0}$ \\
\hline$\overline{\mathbf{H}}$ & $\mathbf{P}$ & $245-000 \mathrm{H}$ & 3241 & 301 & 31 & 9331 & 0 & 0 & $\overline{0}$ \\
\hline$\overline{\mathbf{H}}$ & $\overline{\mathbf{P}}$ & $245-002 \mathrm{H}$ & 160 & 15 & 9 & 135 & ot & 0 & 0 \\
\hline H & $\mathbf{P}$ & $248-010 \mathrm{H}$ & 173 & 16 & 14 & 224 & 0 & 0 & $\overline{0}$ \\
\hline $\mathrm{H}$ & $\mathbf{P}$ & $251-000 \mathrm{H}$ & 1845 & 171 & 37 & 6327 & 0 & 0 & 0 \\
\hline $\mathbf{H}$ & $\mathbf{P}$ & $253-000 \mathrm{H}$ & 1350 & 125 & 8 & 1000 & 0 & 0] & 0 \\
\hline $\mathrm{H}$ & $\mathbf{P}$ & $254-005 \mathrm{H}$ & 816 & 76 & 13 & 988 & 0 & 1 & 1 \\
\hline $\mathrm{H}$ & $\mathbf{P}$ & $254-028 \mathrm{H}$ & 30 & 3 & 9 & 27 & 0 & 0 & 0 \\
\hline $\mathrm{H}$ & $\mathbf{P}$ & $260-001 \mathrm{H}$ & 81 & 8 & 39 & 312 & 0 & 0 & $\overline{0}$ \\
\hline $\mathrm{H}$ & $\mathbf{P}$ & $260-002 \mathrm{H}$ & 81 & 8 & 38 & 304 & 0 & 0 & $\overline{0}$ \\
\hline $\mathrm{H}$ & $P$ & $260-003 \mathrm{H}$ & 100 & 9 & 37 & 333 & 0 & 0 & $\overline{0}$ \\
\hline $\mathrm{H}$ & $\mathbf{P}$ & $260-004 \mathrm{H}$ & 241 & 22 & 36 & 792 & 0 & 0 & 0 \\
\hline $\mathrm{H}$ & $\mathbf{P}$ & $280-001 \mathrm{H}$ & 940 & 87 & 39 & 3393 & 0 & .0 & $\overline{0}$ \\
\hline $\mathrm{H}$ & $\mathbf{P}$ & $281-004 \mathrm{H}$ & 251 & 23 & 39 & 897 & 0 & 0 & 0 \\
\hline $\mathrm{H}$ & $\mathbf{P}$ & $281-006 \mathrm{H}$ & 251 & 23 & 39 & 897 & 0 & 0 & $\overline{0}$ \\
\hline $\mathrm{H}$ & $\mathbf{P}$ & $281-012 \mathrm{H}$ & 112 & 10 & 7 & 70 & 0 & of & 0 \\
\hline $\mathrm{H}$ & $\mathbf{P}$ & $281-013 \mathrm{H}$ & 144 & 13 & 7 & 91 & 0 & 0 & 0 \\
\hline $\mathbf{H}$ & $\mathbf{P}$ & $281-014 \mathrm{H}$ & 144 & 13 & 7 & 91 & 0 & 0] & 0 \\
\hline $\mathbf{H}$ & $\mathbf{P}$ & $281-015 \mathrm{H}$ & 144 & 13 & 7 & 91 & 0 & 0 & 0 \\
\hline $\mathbf{H}$ & $\mathbf{P}$ & $281-016 \mathrm{H}$ & 144 & 13 & 7 & 91 & 0 & 0 & 0 \\
\hline $\mathrm{H}$ & $\mathbf{P}$ & $281-017 \mathrm{H}$ & 144 & 13 & 7 & 91 & 0 & 0 & 0 \\
\hline $\mathbf{H}$ & $\overline{\mathbf{P}}$ & $281-018 H$ & 144 & 13 & 7 & 91 & 0 & 0 & 0 \\
\hline$\overline{\mathrm{H}}$ & $\mathbf{P}$ & $284-000 \mathrm{H}$ & 2500 & 232 & 37 & 8584 & 8 & 1 & 9 \\
\hline $\mathrm{H}$ & $\mathbf{P}$ & $284-001 \mathrm{H}$ & 200 & 19 & 13 & 247 & 0 & 0 & 0 \\
\hline $\mathrm{H}$ & $\mathbf{P}$ & $284-004 \mathrm{H}$ & 840 & 78 & 13 & 1014 & 0 & 이 & 0 \\
\hline $\mathrm{H}$ & $\mathrm{P}$ & $284-005 \mathrm{H}$ & 240 & 22 & 13 & 286 & 0 & 0 & 0 \\
\hline $\mathbf{H}$ & $\mathbf{P}$ & $284-006 \mathrm{H}$ & 240 & 22 & 13 & 286 & 0 & 0 & 0 \\
\hline $\mathrm{H}$ & $\mathrm{P}$ & $284-007 \mathrm{H}$ & 1800 & 167 & 14 & 2338 & 0 & 0 & 0 \\
\hline $\mathrm{H}$ & $\mathbf{P}$ & $285-001 \mathrm{H}$ & 61 & 6 & 30 & 180 & 0 & 0 & 0 \\
\hline $\mathrm{H}$ & $\widehat{P}$ & $285-002 \mathrm{H}$ & 192 & 18 & 27 & 486 & 0 & 0 & 0 \\
\hline
\end{tabular}


Table A-15.--Incipient fire data for $200 \mathrm{H}$ Area

\begin{tabular}{|c|c|c|c|c|c|c|c|c|c|}
\hline \multirow[b]{2}{*}{ Area } & \multirow[b]{2}{*}{ Type } & \multirow[b]{2}{*}{ D } & \multirow[b]{2}{*}{ Area, $\mathrm{ft}^{2}$} & \multirow[b]{2}{*}{ Area, $\mathrm{m}^{2}$} & \multirow{2}{*}{$\begin{array}{c}\text { years in } \\
\text { service to } \\
99\end{array}$} & \multirow[b]{2}{*}{ yrs $^{*} \mathbf{m}^{2}$} & \multicolumn{3}{|c|}{ Fire count } \\
\hline & & & & & & & $\begin{array}{c}\text { Original } \\
\text { count }\end{array}$ & '91 to '94 & $\begin{array}{l}\text { Sum of } \\
\text { all fires }\end{array}$ \\
\hline $\mathrm{H}$ & P & $285-004 \mathrm{H}$ & 44 & 4 & 9 & 36 & 0 & 0 & 0 \\
\hline $\mathrm{H}$ & P & $292-000 \mathrm{H}$ & 13251 & 1231 & 37 & 45547 & 2 & 0 & 2 \\
\hline $\bar{H}$ & P & $292-002 \mathrm{H}$ & 3360 & 312 & 9 & 2808 & 0 & 0 & $\overline{0}$ \\
\hline $\mathrm{H}$ & $\mathrm{P}$ & $292-003 \mathrm{H}$ & 100 & 9 & 9 & 81 & 0 & $\overline{0}$ & $\overline{0}$ \\
\hline H & P & 294-000H & 24000 & 2230 & 9 & 20070 & 0 & 1 & 1 \\
\hline $\mathrm{H}$ & $\mathbf{P}$ & $299-000 \mathrm{H}$ & 1200 & 111 & 12 & 1332 & 0 & 1 & 1 \\
\hline $\mathrm{H}$ & $\mathbf{P}$ & $299-002 \mathrm{H}$ & 576 & 54 & 10 & 540 & 0 & 0 & 0 \\
\hline $\mathrm{H}$ & $\mathbf{P}$ & $299-003 \mathrm{H}$ & 200 & 19 & 9 & 171 & $\overline{0}$ & $\overline{0}$ & $\overline{0}$ \\
\hline $\mathrm{H}$ & P & 299-004H & 1500 & 139 & $\overline{8}$ & 1112 & 0 & 0 & 0 \\
\hline$\overline{\mathrm{H}}$ & $\mathbf{P}$ & $299-005 \mathrm{H}$ & 608 & 56 & $\overline{8}$ & 448 & $\overline{0}$ & $\overline{0}$ & 0 \\
\hline
\end{tabular}


Table A-16.--Incipient fire data for 300M Area

\begin{tabular}{|c|c|c|c|c|c|c|c|c|c|}
\hline \multirow[b]{2}{*}{ Area } & \multirow[b]{2}{*}{ Type } & \multirow[b]{2}{*}{ DD } & \multirow[b]{2}{*}{ Area, $\mathrm{n}^{2}$} & \multirow[b]{2}{*}{ Area, $\mathbf{m}^{2}$} & \multirow{2}{*}{\begin{tabular}{|c|} 
years in \\
service to \\
94 \\
\end{tabular}} & \multirow[b]{2}{*}{$\mathrm{yrs}^{*} \mathrm{~m}^{2}$} & \multicolumn{3}{|c|}{ Fire count } \\
\hline & & & & & & & $\begin{array}{c}\text { Original } \\
\text { count }\end{array}$ & '91 to $94 \mid$ & $\begin{array}{l}\text { Sum of } \\
\text { all fires }\end{array}$ \\
\hline & & 5 & 5n: & 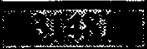 & & 30 & 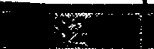 & & $\%$ \\
\hline $\mathbf{M}$ & $\mathbf{P}$ & $305-001 \mathrm{M}$ & 10440 & 970 & 9 & 8730 & of & 1 & 1 \\
\hline$\overline{\mathbf{M}}$ & $\overline{\mathbf{P}}$ & $313-000 \mathrm{M}$ & 57212 & 5315 & 42 & 223230 & 32 & 0 & 32 \\
\hline $\mathbf{M}$ & P & $313-003 \mathrm{M}$ & 44000 & 4088 & 9 & 36792 & 0 & 0 & 0 \\
\hline $\bar{M}$ & $\mathrm{P}$ & $313-006 \mathrm{M}$ & 44000 & 4088 & 9 & 36792 & 0 & 0 & $\overline{0}$ \\
\hline $\mathbf{M}$ & $\mathbf{P}$ & $313-007 \mathrm{M}$ & 360 & 33 & 8 & 264 & 0 & 0 & $\overline{0}$ \\
\hline $\mathbf{M}$ & $\overline{\mathbf{P}}$ & $313-009 \mathrm{M}$ & 315 & 29 & 8 & 232 & 0 & 0 & $\overline{0}$ \\
\hline $\mathbf{M}$ & $\overline{\mathbf{P}}$ & $313-010 \mathrm{M}$ & 144 & 13 & 8 & 104 & 0 & 0 & $\overline{0}$ \\
\hline $\mathbf{M}$ & $\mathbf{P}$ & 313-011M & 192 & 18 & 8 & 144 & 0 & 0 & $\overline{0}$ \\
\hline $\mathbf{M}$ & $\mathbf{P}$ & $315-000 \mathrm{M}$ & 15286 & 1420 & 19 & 26980 & 0 & 0 & $\overline{0}$ \\
\hline $\mathbf{M}$ & $\mathbf{P}$ & $315-001 \mathrm{M}$ & 72 & 7 & 19 & 133 & 0 & 0 & $\overline{0}$ \\
\hline$\overline{\mathbf{M}}$ & $\bar{P}$ & $315-002 \mathrm{M}$ & 100 & 9 & 19 & 171 & 0 & † & $\overline{0}$ \\
\hline $\mathbf{M}$ & P & $316-000 \mathrm{M}$ & 1600 & 149 & 7 & 1043 & 0 & 0 & $\overline{0}$ \\
\hline $\mathbf{M}$ & $\mathbf{P}$ & $319-000 \mathrm{M}$ & 506 & 47 & 37 & 1739 & 0 & 0 & $\overline{0}$ \\
\hline $\mathbf{M}$ & $\mathbf{P}$ & $320-000 \mathrm{M}$ & 63288 & 5880 & 37 & 217560 & 17 & 3 & 20 \\
\hline $\mathbf{M}$ & $\mathbf{P}$ & $320-001 \mathrm{M}$ & 720 & 67 & 9 & 603 & 0 & 0 & 0 \\
\hline $\mathbf{M}$ & $\mathbf{P}$ & $321-000 \mathrm{M}$ & 56072 & 5209 & 37 & $1 \subseteq 2733$ & 16 & 1 & 17 \\
\hline $\mathbf{M}$ & P & $321-002 \mathrm{M}$ & 510 & 47 & 9 & 423 & 의 & 0 & 0 \\
\hline $\mathbf{M}$ & $\mathbf{P}$ & $321-003 \mathrm{M}$ & 4000 & 372 & 11 & 4092 & 0 & 0 & 0 \\
\hline $\mathbf{M}$ & P & $321-004 \mathrm{M}$ & 240 & 22 & 9 & 198 & 0 & 0 & 0 \\
\hline $\bar{M}$ & $\mathbf{P}$ & $322-000 \mathrm{M}$ & 7812 & 726 & 37 & 26862 & 16 & 0 & 16 \\
\hline $\mathbf{M}$ & $\bar{P}$ & $322-001 \mathrm{M}$ & 190 & 18 & 21 & 378 & 0 & 0 & $\overline{0}$ \\
\hline $\mathbf{M}$ & $\mathbf{P}$ & $322-002 \mathrm{M}$ & 1104 & 103 & 14 & $\overline{1442}$ & 0 & 0 & $\overline{0}$ \\
\hline $\mathbf{M}$ & $\bar{P}$ & $322-003 \mathrm{M}$ & 80 & 7 & 14 & 98 & 0 & 0 & $\overline{0}$ \\
\hline $\bar{M}$ & $\mathbf{P}$ & $323-000 \mathrm{M}$ & 360 & 33 & 8 & 264 & 0 & 0 & 0 \\
\hline $\mathbf{M}$ & P & $324-000 \mathrm{M}$ & 1875 & 174 & 28 & 4872 & 1 & 0 & 1 \\
\hline $\bar{M}$ & $\bar{P}$ & $330-000 \mathrm{M}$ & 8000 & 743 & 5 & $\overline{3715}$ & 0 & 0 & $\overline{0}$ \\
\hline $\mathbf{M}$ & P & $331-000 \mathrm{M}$ & 8000 & 743 & 6 & 4458 & 0 & 0 & $\overline{0}$ \\
\hline$\overline{\mathbf{M}}$ & $\bar{P}$ & $340-000 \mathrm{M}$ & 400 & 37 & 6 & 222 & 0 & 0 & $\overline{0}$ \\
\hline $\mathbf{M}$ & P & $341-000 \mathrm{M}$ & 6000 & 557 & 6 & 3342 & 0 & 0 & 0 \\
\hline $\bar{M}$ & P & $341-001 \mathrm{M}$ & 5600 & 520 & 6 & 3120 & 0 & 0 & 0 \\
\hline $\mathbf{M}$ & $\bar{P}$ & $341-005 \mathrm{M}$ & 400 & 37 & 7 & 259 & 0 & 0 & $c$ \\
\hline & & 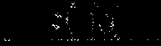 & 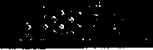 & & & 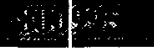 & & 8 & \\
\hline
\end{tabular}


Table A-16.--Incipient fire data for $T$ Area

\begin{tabular}{|c|c|c|c|c|c|c|c|c|c|}
\hline \multirow[b]{2}{*}{ Area } & \multirow[b]{2}{*}{ Type } & \multirow[b]{2}{*}{ ID } & \multirow[b]{2}{*}{ Area, $\mathrm{f}^{2}$} & \multirow[b]{2}{*}{ Area, $\mathbf{m}^{2}$} & \multirow{2}{*}{$\begin{array}{c}\text { years in } \\
\text { service to } \\
94\end{array}$} & \multirow[b]{2}{*}{$\mathrm{yrs}^{*} \mathrm{~m}^{2}$} & \multicolumn{3}{|c|}{ Fire count } \\
\hline & & & & & & & $\begin{array}{c}\text { Original } \\
\text { count }\end{array}$ & $\mid \cdot 91$ to '94| & $\begin{array}{l}\text { Sum of } \\
\text { all fires }\end{array}$ \\
\hline & & 8 & Ds: & 78 & & 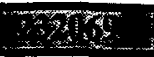 & & & \\
\hline $\mathbf{T}$ & $\mathbf{P}$ & $607-41 \mathrm{~T}$ & 400 & 37 & 81 & 296 & 0 & 0 & 0 \\
\hline$T$ & $\mathbf{P}$ & $607-46 \mathrm{~T}$ & 600 & 56 & 6 & 336 & 0 & 0 & 0 \\
\hline $\mathbf{T}$ & $\mathbf{P}$ & $669-\mathrm{T}$ & 3150 & 293 & 8 & 2344 & 0 & 0 & 0 \\
\hline $\mathrm{T}$ & $\mathbf{P}$ & $670-T$ & 3600 & 334 & 8 & 2672 & 0 & 0 & 0 \\
\hline $\mathbf{T}$ & $\mathbf{P}$ & $671-\mathrm{T}$ & 200 & 19 & 12 & 228 & 0 & 0 & 0 \\
\hline $\mathbf{T}$ & $\mathbf{P}$ & 672-T & 12120 & 1126 & 11 & 12386 & $\overline{0}$ & 0 & 0 \\
\hline $\mathbf{T}$ & $\mathbf{P}$ & 673-T & 4800 & 446 & 12 & 5352 & $\overline{0}$ & 0 & $\overline{0}$ \\
\hline $\mathbf{T}$ & $\mathbf{P}$ & 674-T & 7000 & 650 & 13 & 8450 & $\overline{0}$ & 0 & $\overline{0}$ \\
\hline $\mathbf{T}$ & $\mathbf{P}$ & 675-T & 6642 & 617 & 12 & 7404 & 1 & 0 & 1 \\
\hline $\mathbf{T}$ & $\mathbf{P}$ & $676-10 \mathrm{~T}$ & 1536 & 143 & 7 & 1001 & $\overline{0}$ & 0 & 0 \\
\hline $\mathbf{T}$ & $\mathbf{P}$ & $676-11 \mathrm{~T}$ & 1536 & 143 & 5 & 715 & $\overline{0}$ & 0 & 0 \\
\hline $\mathbf{T}$ & $\mathbf{P}$ & $676-12 \mathrm{~T}$ & 1536 & 143 & 6 & 858 & 0 & 0 & 0 \\
\hline $\mathbf{T}$ & $\mathbf{P}$ & 676-14T & 1792 & 166 & 6 & 996 & 0 & 0 & 0 \\
\hline $\mathbf{T}$ & $\mathbf{P}$ & 676-15T & 1792 & 166 & 5 & 830 & 0 & 0 & 0 \\
\hline $\mathbf{T}$ & $\mathbf{P}$ & $676-1 \mathrm{~T}$ & 1536 & 143 & 14 & 2002 & 0 & 0 & 0 \\
\hline $\mathbf{T}$ & $\mathbf{P}$ & $676-2 \mathrm{~T}$ & 720 & 67 & 9 & 603 & $\overline{0}$ & 0 & 0 \\
\hline $\mathbf{T}$ & $\mathbf{P}$ & $676-8 \mathrm{~T}$ & 1536 & 143 & 9 & 1287 & 0 & 0 & 0 \\
\hline $\mathrm{T}$ & $\mathbf{P}$ & $676-9 \mathrm{~T}$ & 1536 & 143 & 6 & 858 & 0 & $\overrightarrow{0}$ & 0 \\
\hline $\mathbf{T}$ & $\overline{\mathbf{P}}$ & 676-T & 1536 & 143 & 14 & 2002 & $\overline{0}$ & 0 & $\overline{0}$ \\
\hline $\mathbf{T}$ & $\mathbf{P}$ & $677-1 \mathrm{~T}$ & 192 & 18 & 14 & 252 & $\overline{0}$ & 0 & 0 \\
\hline $\mathbf{T}$ & $\mathbf{P}$ & $677-2 \mathrm{~T}$ & 192 & 18 & 14 & 252 & 0 & 0 & 0 \\
\hline $\mathbf{T}$ & $\mathbf{P}$ & $677-T$ & 15280 & 1420 & 37 & 52540 & 0 & 0 & 0 \\
\hline $\mathbf{T}$ & $\mathbf{P}$ & 678-T & 12957 & 1204 & 37 & 44548 & 4 & 0 & 4 \\
\hline $\mathbf{T}$ & $\mathbf{P}$ & $679-2 \mathrm{~T}$ & 120 & 11 & 9 & 99 & 0 & 0 & 0 \\
\hline $\mathbf{T}$ & $\mathbf{P}$ & $679-5 \mathrm{~T}$ & 48 & 4 & 9 & 36 & 0 & 0 & 0 \\
\hline $\mathbf{T}$ & $\mathbf{P}$ & $679.7 \mathrm{~T}$ & 101 & 9 & 9 & 81 & 0 & 0 & 0 \\
\hline $\mathrm{T}$ & P & $679-8 \mathrm{~T}$ & 101 & 9 & 9 & 81 & 0 & 0 & 0 \\
\hline $\mathbf{T}$ & $\mathbf{P}$ & 679-T & 13360 & 1241 & 41 & 50881 & 0 & 0 & 0 \\
\hline $\mathbf{T}$ & $\mathbf{P}$ & 682-T & 500 & 46 & 6 & 276 & 0 & 0 & 0 \\
\hline $\mathrm{T}$ & $\mathbf{P}$ & 684-T & 420 & 39 & 7 & 273 & 0 & 0 & 0 \\
\hline $\mathbf{T}$ & $\mathbf{P}$ & $704-1 \mathrm{~T}$ & 6500 & 604 & 7 & 4228 & 0 & 0 & 0 \\
\hline $\mathbf{T}$ & P & 704-T & 6395 & 594 & 12 & 7128 & 0 & 0 & 0 \\
\hline $\mathbf{T}$ & $\bar{P}$ & $7711-T$ & 200 & 19 & 10 & 190 & 0 & 0 & 0 \\
\hline $\mathbf{T}$ & $\overline{\mathbf{P}}$ & $7772-T$ & 40000 & 3716 & 5 & 18580 & 1 & 0 & 1 \\
\hline $\mathbf{T}$ & P & $904-\mathrm{T}$ & 3600 & 334 & 6 & 2004 & 0 & 0 & 0 \\
\hline & & & 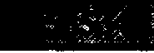 & 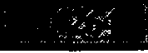 & & & 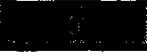 & & \\
\hline
\end{tabular}


Table A-17.--Incipient fire data for S Area

\begin{tabular}{|c|c|c|c|c|c|c|c|c|c|}
\hline \multirow[b]{2}{*}{ Area } & \multirow[b]{2}{*}{ Type } & \multirow[b]{2}{*}{ ID } & \multirow[b]{2}{*}{ Area, ft2 } & \multirow[b]{2}{*}{ Area, $\mathbf{m} 2$} & \multirow{2}{*}{$\begin{array}{c}\text { years in } \\
\text { service to } \\
' 94\end{array}$} & \multirow[b]{2}{*}{ yrs: ${ }^{*} \mathbf{m} 2$} & \multicolumn{3}{|c|}{ Fire count } \\
\hline & & & & & & & $\begin{array}{c}\text { Original } \\
\text { count }\end{array}$ & '91 to '94| & $\begin{array}{l}\text { Sum of } \\
\text { all fires }\end{array}$ \\
\hline & & ST: & हn! & 5 & & 76 & & & \\
\hline $\mathbf{S}$ & $\mathbf{P}$ & $210-000 \mathrm{~s}$ & 93600 & 8696 & 4 & 34784 & 0 & 1 & 1 \\
\hline $\bar{S}$ & $\mathbf{P}$ & $221-000 \mathrm{~S}$ & 191400 & 17782 & 4 & 71128 & 0 & 2 & 2 \\
\hline $\mathbf{S}$ & $\mathbf{P}$ & $250-000 \mathrm{~S}$ & 24824 & 2306 & 4 & 9224 & 0 & 0 & 0 \\
\hline $\bar{S}$ & $\mathbf{P}$ & $250-0015$ & 2400 & 223 & 4 & 892 & 0 & 0 & 0 \\
\hline $\mathbf{S}$ & $\bar{P}$ & $250-002 S$ & 2000 & 186 & 4] & 744 & 0 & 0 & 0 \\
\hline $\mathbf{S}$ & $\mathbf{P}$ & $250-003 \mathrm{~S}$ & 200 & 19 & 4 & 76 & 0 & 0 & 0 \\
\hline $\bar{S}$ & P & $260-0005$ & & 0 & 4 & 0 & 0 & 0 & 0 \\
\hline $\mathbf{S}$ & $\mathbf{P}$ & $260-0015$ & 288 & 27 & 4 & 108 & 0 & 0 & $\overline{0}$ \\
\hline $\bar{S}$ & $\overline{\mathbf{P}}$ & $263-155 S$ & 200 & 19 & 4 & 76 & 0 & 0 & 0 \\
\hline $\mathbf{S}$ & $\bar{P}$ & $291-000 \mathrm{~S}$ & 0 & 0 & 4 & 0 & 0 & $\overline{0}$ & 0 \\
\hline $\mathbf{S}$ & $\mathbf{P}$ & $292-0005$ & 21936 & 2038 & 4 & 8152 & 0 & 0 & 0 \\
\hline $\mathbf{S}$ & $\mathbf{P}$ & $375-000 \mathrm{~S}$ & 0 & 0 & 4 & 0 & 0 & 0 & 0 \\
\hline $\mathbf{S}$ & $\mathbf{P}$ & $422-0005$ & 4611 & 428 & 4 & 1712 & 0 & 0 & 0 \\
\hline $\mathbf{S}$ & $\mathbf{P}$ & $422-0015$ & 0 & 0 & 4 & 0 & 0 & 0 & 0 \\
\hline $\mathbf{S}$ & $\bar{P}$ & $422-0025$ & 4200 & 390 & 4 & 1560 & 0 & 0 & 0 \\
\hline $\mathbf{S}$ & $\bar{P}$ & $422-003 \mathrm{~S}$ & 460 & 43 & 2 & 86 & 0 & 0 & 0 \\
\hline $\mathbf{S}$ & $\mathbf{P}$ & $422-004 S$ & 1075 & 100 & 4 & 400 & 0 & 0 & 0 \\
\hline $\mathbf{S}$ & $\overline{\mathbf{P}}$ & $422-005 S$ & 0 & 0 & 4 & 0 & 0 & 0 & 0 \\
\hline $\mathbf{S}$ & $\bar{P}$ & $422-0065$ & 576 & 54 & 4 & 216 & 0 & 0 & 0 \\
\hline$S$ & $\mathrm{P}$ & $430-000 \mathrm{~S}$ & 0 & 0 & 4 & 0 & 0 & 0 & 0 \\
\hline $\mathbf{S}$ & $\overline{\mathbf{P}}$ & $430-0015$ & 304 & 28 & 4 & 112 & 0 & 0 & 0 \\
\hline $\bar{S}$ & P & $430-003 \mathrm{~s}$ & 828 & 77 & (4) & 308 & 0 & 0 & 0 \\
\hline $\mathbf{S}$ & P & $430-004 S$ & 527 & 49 & 4 & 196 & $\overline{0}$ & $\overline{0}$ & $\overline{0}$ \\
\hline $\mathbf{S}$ & $\mathbf{P}$ & $511-0025$ & 48 & 4 & 4 & 16 & $\overline{0}$ & 0 & 0 \\
\hline S & $\bar{P}$ & $511-0035$ & 200 & 19 & 4 & 76 & $\overline{0}$ & $\overline{0}$ & 0 \\
\hline $\mathbf{S}$ & $\mathrm{P}$ & $512-000 \mathrm{~S}$ & 7215 & 670 & 4 & 2680 & 0 & 0 & 0 \\
\hline S & $\vec{P}$ & $512-003 S$ & 240 & 22 & 4 & 88 & $\overline{0}$ & $\overline{0}$ & $\overrightarrow{0}$ \\
\hline $\mathbf{S}$ & P & $512-004 S$ & 1352 & 126 & 4 & 504 & $\overline{0}$ & $\overline{0}$ & $\overline{0}$ \\
\hline$S$ & $\mathrm{P}$ & $512-005 S$ & 1344 & 125 & 4 & 500 & 0 & 0 & 0 \\
\hline S & $\mathbf{P}$ & $512-006 \mathrm{~S}$ & 800 & 74 & 4 & 296 & 0 & 0 & 0 \\
\hline $\mathbf{S}$ & $\mathbf{P}$ & $512-007 \mathrm{~s}$ & 800 & 74 & 4 & 296 & $\overline{0}$ & 0 & 0 \\
\hline $\mathbf{S}$ & $\mathbf{P}$ & $512-008 S$ & 800 & 74 & 4 & 296 & $\overline{0}$ & $\overline{0}$ & 0 \\
\hline S & $\mathbf{P}$ & $512-009 \mathrm{~S}$ & 960 & 89 & 4 & 356 & $\overline{0}$ & $\overline{0}$ & 0 \\
\hline$S$ & $\mathbf{P}$ & $512-0105$ & 960 & 89 & 4 & 356 & 0 & $\overline{0}$ & 0 \\
\hline $\mathbf{S}$ & $\mathbf{P}$ & $512-0115$ & 960 & 89 & 4 & 356 & 0 & 0 & 0 \\
\hline $\mathbf{S}$ & $\mathbf{P}$ & $701-000 \mathrm{~s}$ & 1000 & 93 & 4 & 372 & 0 & 0 & 0 \\
\hline $\bar{S}$ & $\bar{P}$ & $701-002 S$ & 36 & 3 & 4 & 12 & 0 & 0 & 0 \\
\hline S & $\bar{P}$ & $701-003 S$ & 128 & 12 & 4 & 48 & 0 & 0 & 0 \\
\hline$S$ & P & $702-000 \mathrm{~S}$ & 1000 & 93 & 4 & 372 & 0 & $\overline{0}$ & 0 \\
\hline $\mathbf{S}$ & $\mathbf{P}$ & $704-0005$ & 25844 & 2401 & & 0 & 0 & 1 & 1 \\
\hline S & $P$ & $704-0015$ & 1504 & 140 & 4 & 560 & $\overline{0}$ & 0 & 0 \\
\hline $\mathbf{S}$ & $\mathbf{P}$ & $704-002 S$ & 1411 & 131 & 4 & 524 & 0 & 0 & 0 \\
\hline $\mathbf{S}$ & $\mathbf{P}$ & $704-003 \mathrm{~S}$ & 1398 & 130 & 4 & 520 & 0 & 0 & 0 \\
\hline $\mathbf{S}$ & $\mathbf{P}$ & $704-004 S$ & 1396 & 130 & 4 & 520 & 0 & 0 & 0 \\
\hline $\mathbf{S}$ & $\bar{P}$ & $704-005 \mathrm{~S}$ & 1365 & 127 & 4 & 508 & 0 & 0 & 0 \\
\hline$S$ & $\mathbf{P}$ & $704-0065$ & $\overline{1447}$ & 134 & 4 & 536 & 0 & 0 & 0 \\
\hline
\end{tabular}


Table A-17.--Incipient fire data for $S$ Area

\begin{tabular}{|c|c|c|c|c|c|c|c|c|c|}
\hline \multirow[b]{2}{*}{ Area } & \multirow[b]{2}{*}{ Type } & \multirow[b]{2}{*}{ ID } & \multirow[b]{2}{*}{ Area, ft2 } & \multirow[b]{2}{*}{ Area, m2 } & \multirow{2}{*}{\begin{tabular}{|c|} 
years in \\
service to \\
'94
\end{tabular}} & \multirow[b]{2}{*}{ yrs*m2 } & \multicolumn{3}{|c|}{ Fire count } \\
\hline & & & & & & & $\begin{array}{c}\text { Original } \\
\text { count }\end{array}$ & '91 to 94 & $\begin{array}{l}\text { Sum of } \\
\text { all fires }\end{array}$ \\
\hline$S$ & $P$ & 704-007S & 1440 & 134 & 4 & 536 & 0 & 0 & 0 \\
\hline $\mathbf{S}$ & $\mathbf{P}$ & 704-008S & 1498 & 139 & 41 & 556 & 0 & 0 & 0 \\
\hline $\mathbf{S}$ & P & 704-015S & 896 & 83 & 4 & 332 & 0 & 0 & 0 \\
\hline$S$ & $P$ & $704-016 \mathrm{~S}$ & 1116 & 104 & 4 & 416 & of & of & 0 \\
\hline S & $\mathbf{P}$ & 704-018S & 1296 & 120 & 4[ & 480 & 요 & of & 0 \\
\hline S & $\mathbf{P}$ & 704-019s & 827 & 77 & 4 & 308 & 의 & of & 0 \\
\hline $\mathbf{S}$ & $\mathbf{P}$ & 704-020S & 1393 & 129 & 4 & 516 & 0 & 0 & 0 \\
\hline $\mathbf{S}$ & $\mathbf{P}$ & $704-024 \mathrm{~S}$ & 686 & 64 & $4 \mid$ & 256 & of & 0 & 0 \\
\hline $\mathbf{S}$ & $\mathbf{P}$ & $704-025 S$ & 1736 & 161 & 4 & 644 & 0] & 11 & 1 \\
\hline $\mathbf{S}$ & $\mathbf{P}$ & $704-026 \mathrm{~S}$ & 1731 & 161 & 4 & 644 & 0 & 0 & 0 \\
\hline $\mathbf{S}$ & $\mathbf{P}$ & 704-027S & 1731| & 161 & 4 & 644 & of & 0 & 의 \\
\hline$S$ & $\mathbf{P}$ & $704-0285$ & 1731 & 161 & \begin{tabular}{l|l}
4 \\
\end{tabular} & 644 & of & 0 & 0 \\
\hline S & $\mathbf{P}$ & $704-029 \mathrm{~S}$ & 1736 & 161 & 4] & 644 & 0] & 0 & 0 \\
\hline $\mathbf{S}$ & $\mathbf{P}$ & $704-030 \mathrm{~S}$ & 1731 & 161 & 4 & 644 & 0 & 0 & 0) \\
\hline $\mathbf{S}$ & $\mathbf{P}$ & 704-031S & 1731 & 161 & 4 & 644 & 0 & 0 & 0 \\
\hline $\mathbf{S}$ & $\mathbf{P}$ & $704-032 \mathrm{~S}$ & 200 & 19 & 3 & 57 & 0 & 0 & 0 \\
\hline $\mathbf{S}$ & I & $704-035 S$ & 1754 & 163 & 4 & 652 & 0] & 0 & 0 \\
\hline $\mathbf{S}$ & P & $704-041 \mathrm{~S}$ & 660 & 61 & 4 & 244 & 0 & 0 & 0 \\
\hline$S$ & $P$ & $704-042 S$ & 660 & 61 & 4 & 244 & 0 & 0 & 0 \\
\hline$\underline{S}$ & $P$ & $704-044 S$ & 720 & 67| & 4 & 268 & of & 0 & 0 \\
\hline$S$ & $P$ & $704-045 S$ & 1440 & 134 & 4 & 536 & 0 & 0 & 0 \\
\hline$S$ & $P$ & $704-046 \mathrm{~S}$ & 1440 & 134 & 4 & 536 & of & 0 & 0 \\
\hline $\mathbf{S}$ & $\mathbf{P}$ & $704-047 \mathrm{~S}$ & 240 & 22 & $4 \mid$ & 88 & 요 & 의 & 0 \\
\hline $\mathbf{S}$ & $\mathbf{P}$ & $704-049 S$ & 5369 & 499 & 2 & 998 & 요 & 의 & 의 \\
\hline S & P & $704-050 \mathrm{~s}$ & 960 & 89 & 4 & 356 & 어 & 요 & 의 \\
\hline $\mathbf{S}$ & $\mathbf{P}$ & 704-051S & 960 & 89 & $4 \mid$ & 356 & 요 & 의 & 의 \\
\hline$\underline{S}$ & $\mathrm{P}$ & $704-052 S$ & 960 & 89 & 4 & 356 & 0 & 0 & 0 \\
\hline s & $P$ & $704-054 S$ & 240 & 22 & 4 & 88 & 0 & of & 0 \\
\hline S & $\mathbf{P}$ & $704-058 \mathrm{~S}$ & 1513 & 141 & 4 & 564 & 0 & 0 & 의 \\
\hline S & $P$ & $704-059 \mathrm{~S}$ & 792 & 74 & 4 & 296 & 0 & 0 & 0 \\
\hline S & $P$ & $704-060 \mathrm{~S}$ & 1492 & 139 & 4 & 556 & 0 & 0 & 0 \\
\hline$S$ & $P$ & $704-0615$ & 1703 & 158 & 5 & 790 & 0 & 0 & 0 \\
\hline $\mathbf{S}$ & $\mathbf{P}$ & $704-062 \mathrm{~S}$ & 960 & 89 & 4 & 356 & 0 & 0 & 0 \\
\hline$S$ & $P$ & $704-063 S$ & 960 & 89 & 4 & 356 & 0 & 요 & 0 \\
\hline$s$ & $\mathbf{P}$ & $704-064 S$ & 960 & 89 & 4 & 356 & 0 & 0 & 0 \\
\hline $\mathbf{S}$ & P & $704-065 \mathrm{~S}$ & 960 & 89 & 4 & 356 & 오 & 의 & 의 \\
\hline S & $\mathbf{P}$ & $704-066 \mathrm{~S}$ & 1909 & 177| & 4 & 708 & 0 & 요 & 0 \\
\hline$\underline{\mathbf{S}}$ & P & $704-067 \mathrm{~S}$ & 1909 & 177 & 4 & 708 & 0 & of & 0 \\
\hline $\mathbf{S}$ & $\mathbf{P}$ & $704-068 \mathrm{~S}$ & 1347 & 125 & 4 & 500 & 0 & 0 & 0 \\
\hline $\mathbf{S}$ & $\mathbf{P}$ & $704-071 \mathrm{~S}$ & 21204 & 1970 & 4) & 7880 & 0 & 11 & 1 \\
\hline $\mathbf{S}$ & P & $704-072 S$ & 19900 & 1849 & 41 & 7396 & 0 & of & 0 \\
\hline $\mathbf{S}$ & $\mathbf{P}$ & $704-074 S$ & 1417 & 132 & 4[ & 528 & 이 & 0 & 0 \\
\hline $\mathbf{S}$ & $P$ & $704-081 S$ & 720 & 67 & 4 & 268 & 의 & 0 & 0 \\
\hline $\mathbf{S}$ & $P$ & $704-082 S$ & 1440 & 134 & 4 & 536 & 0 & 요 & 0 \\
\hline $\mathbf{s}$ & $\mathbf{P}$ & 704-089S & 720 & 67) & 4 & 268 & 0 & 0 & 0 \\
\hline S & P & $704-096 \mathrm{~S}$ & 80 & 7. & 4 & 28 & 0 & 0 & 0 \\
\hline $\mathbf{S}$ & $\mathbf{P}$ & $704-097 \mathrm{~S}$ & 960 & 89 & 4[ & 356 & 0 & 0] & 0 \\
\hline
\end{tabular}


Table A-17.--Incipient fire data for S Area

\begin{tabular}{|c|c|c|c|c|c|c|c|c|c|}
\hline \multirow[b]{2}{*}{ Area } & \multirow[b]{2}{*}{ Type } & \multirow[b]{2}{*}{ ID } & \multirow[b]{2}{*}{ Area, $\mathrm{ft2}$} & \multirow[b]{2}{*}{ Area, $\mathbf{m} 2$} & \multirow{2}{*}{$\begin{array}{c}\text { years in } \\
\text { service to } \\
94\end{array}$} & \multirow[b]{2}{*}{ yrsi*m2 } & \multicolumn{3}{|c|}{ Fire count } \\
\hline & & & & & & & $\begin{array}{c}\text { Original } \\
\text { count }\end{array}$ & '91 to '94 & $\begin{array}{l}\text { Sum of } \\
\text { all fires }\end{array}$ \\
\hline $\mathbf{S}$ & $\mathbf{P}$ & $704-098 \mathrm{~S}$ & 1339 & 124 & 4 & 496 & 0 & 0 & 0 \\
\hline S & $\overline{\mathbf{P}}$ & $704-099 \mathrm{~S}$ & 889 & 83 & 4 & 332 & 0 & 0 & 0 \\
\hline $\bar{S}$ & P & $704-111 \mathrm{~S}$ & 0 & 0 & 4 & $\overline{0}$ & 0 & 0 & 0 \\
\hline$S$ & $\mathbf{P}$ & $704-112 \mathrm{~S}$ & 1792 & 166 & 4 & 664 & 이 & 0 & 0 \\
\hline $\mathbf{S}$ & P & $704-115 S$ & 1953 & 181 & 4 & 724 & 0 & 0 & 0 \\
\hline $\mathbf{S}$ & $\mathbf{P}$ & $704-116 S$ & 288 & 27 & 4 & 108 & 0 & 0 & 0 \\
\hline S & $\overline{\mathbf{P}}$ & $704-1185$ & 960 & 89 & 4 & 356 & 0 & 0 & 0 \\
\hline S & $\mathrm{P}$ & $704-119 S$ & 720 & 67 & 4 & 268 & 0 & 0 & 0 \\
\hline$S$ & $P$ & $704-120 S$ & 700 & 65 & 4 & 260 & 0 & 0 & 0 \\
\hline S & $\mathbf{P}$ & 704-13S & 312 & 29 & 4 & 116 & 이 & 0 & 0 \\
\hline $\mathbf{S}$ & $\mathbf{P}$ & $706-000 \mathrm{~S}$ & 8640 & 803 & 2 & 1606 & of & 0 & 0 \\
\hline $\mathbf{S}$ & P & $707-000 \mathrm{~S}$ & 4500 & 418 & 4 & 1672 & 0 & 0 & 0 \\
\hline $\bar{S}$ & $\bar{P}$ & $707-0015$ & 480 & 45 & 4 & 180 & 0 & 0 & 0 \\
\hline $\mathbf{S}$ & P & $707-002 \mathrm{~S}$ & 360 & 33 & 4 & 132 & 0 & 0 & 0 \\
\hline S & $\overline{\mathbf{P}}$ & $707-0035$ & 160 & 15 & 4 & 60 & 0 & 0 & 0 \\
\hline $\mathbf{S}$ & $\mathbf{P}$ & $707-004 S$ & 160 & 15 & 4 & 60 & 0 & 0 & 0 \\
\hline $\mathbf{S}$ & $P$ & $707.005 S$ & 200 & 19 & 4 & 76 & 0 & 0 & 0 \\
\hline $\mathbf{S}$ & $\mathrm{P}$ & $714-000 \mathrm{~S}$ & 12000 & 1115 & 4 & 4460 & 0 & 0 & 0 \\
\hline $\mathbf{S}$ & $\mathbf{P}$ & $714-001 \mathrm{~s}$ & 1040 & 97 & 4 & 388 & 이 & 이 & 0 \\
\hline $\mathbf{S}$ & $\mathbf{P}$ & $714-002 \mathrm{~s}$ & 954 & 89 & 4 & 356 & 0 & 0 & 0 \\
\hline $\mathbf{S}$ & $\mathbf{P}$ & 714-003s & 954 & 89 & 4 & 356 & 0 & 0 & 0 \\
\hline $\mathbf{S}$ & P & $714-004 S$ & 954 & 89 & 4 & 356 & 0 & of & 0 \\
\hline $\bar{S}$ & $\mathbf{P}$ & $714-005 \mathrm{~S}$ & 954 & 89 & 4 & 356 & 이 & 0 & 0 \\
\hline S & $\mathbf{P}$ & $714-006 \mathrm{~S}$ & 960 & 89 & 4 & 356 & of & 0 & 0 \\
\hline$S$ & $\bar{P}$ & $717-000 \mathrm{~S}$ & 2000 & 186 & 4 & 744 & 0 & 0 & 0 \\
\hline $\mathbf{S}$ & $\mathbf{P}$ & $717-001 \mathrm{~s}$ & 240 & 22 & 4 & 88 & 0 & 0 & 0 \\
\hline S & P & $717-0035$ & 756 & 70 & 4 & 280 & 0 & 0 & 0 \\
\hline S & $\mathbf{P}$ & $717-011 \mathrm{~s}$ & 9600 & 892 & 4 & 3568 & 0 & 0 & 0 \\
\hline S & $\mathbf{P}$ & $717-0125$ & 8000 & 743 & 4 & 2972 & 0 & 0 & 0 \\
\hline $\bar{S}$ & $\bar{P}$ & $717-0135$ & 10800 & 1003 & 4 & 4012 & 0 & 0 & 0 \\
\hline $\bar{S}$ & $\bar{P}$ & $981-000 \mathrm{~S}$ & 2430 & 226 & 4 & 904 & 0 & 0 & 0 \\
\hline & & $D=$ & wis & & & & & & \\
\hline
\end{tabular}


Table A-18.--Incipient fire data for G,N,E Area

\begin{tabular}{|c|c|c|c|c|c|c|c|c|c|}
\hline \multirow{2}{*}{ Area } & \multirow[b]{2}{*}{ Type } & \multirow[b]{2}{*}{ D } & \multirow[b]{2}{*}{ Area, $\mathrm{ft}^{2}$} & \multirow[b]{2}{*}{ Area, $\mathbf{m}^{2}$} & \multirow{2}{*}{\begin{tabular}{|c|} 
years in \\
service to \\
94
\end{tabular}} & \multirow[b]{2}{*}{$\mathrm{yrs}^{*} \mathrm{~m}^{2}$} & \multicolumn{3}{|c|}{ Fire count } \\
\hline & & & & & & & $\begin{array}{c}\text { Original } \\
\text { count }\end{array}$ & $\mid$ '91 to '94| & $\begin{array}{l}\text { Sum of } \\
\text { all fires }\end{array}$ \\
\hline & & MT: & 1355 & WT & & FATS & $\pi$ & & $\pi$ \\
\hline$E$ & $\mathbf{S}$ & 643-000E & 0 & 0 & 0 & 0 & 0 & 2 & 2 \\
\hline $\mathbf{G}$ & $\mathbf{S}$ & 504-001G & 576 & 54 & 37 & 1998 & 0 & 0 & 0 \\
\hline G & $\mathbf{S}$ & $504-002 \mathrm{G}$ & 576 & 54 & 37 & 1998 & $\overline{0}$ & 0 & $\overline{0}$ \\
\hline $\mathbf{G}$ & $\mathbf{S}$ & $504-003 G$ & 756 & 70 & 37 & 2590 & 0 & 0 & 0 \\
\hline $\bar{G}$ & $\bar{S}$ & $607-038 \mathrm{G}$ & 600 & 56 & 9 & 504 & $\overline{0}$ & 0 & 0 \\
\hline$G$ & $\mathbf{S}$ & $608-000 \mathrm{G}$ & 1477 & 137 & 41 & 5617 & 0 & 0 & 0 \\
\hline $\mathbf{G}$ & $S$ & $609-000 \mathrm{G}$ & 4000 & 372 & 38 & 14136 & 0 & 0 & 0 \\
\hline $\bar{G}$ & S & 614-006G & 86 & 8 & 41 & 328 & $\overline{0}$ & 0 & 0 \\
\hline G & S & $614-048 \mathrm{G}$ & 93 & 9 & 5 & 45 & 0 & 0 & 0 \\
\hline G & $\mathbf{S}$ & 614-049G & 93 & 9 & 5 & 45 & $\overline{0}$ & 0 & 0 \\
\hline G & $S$ & 614-050G & 93 & 9 & 5 & 45 & 0 & 0 & $\overline{0}$ \\
\hline $\bar{G}$ & S & $614-051 G$ & 93 & 9 & 5 & 45 & $\overline{0}$ & 0 & $\overline{0}$ \\
\hline $\bar{G}$ & S & $614-052 G$ & 93 & 9 & 5 & 45 & $\overline{0}$ & 0 & $\overline{0}$ \\
\hline $\bar{G}$ & $S$ & $614-053 G$ & 93 & 9 & 5 & 45 & 0 & 0 & 0 \\
\hline G & $S$ & $614-054 G$ & 93 & 9 & 5 & 45 & 0 & 0 & 0 \\
\hline $\bar{G}$ & S & $614-055 G$ & 93 & 9 & 5 & 45 & $\overline{0}$ & $\overline{0}$ & $\overline{0}$ \\
\hline $\mathbf{G}$ & $S$ & 616-000G & 654 & 61 & 41 & 2501 & 0 & 0 & 0 \\
\hline G & $S$ & $617.000 \mathrm{G}$ & 2400 & 223 & 10 & 2230 & 0 & 0 & 0 \\
\hline G & S & $617-002 \mathrm{G}$ & 480 & 45 & 8 & 360 & 0 & 0 & 0 \\
\hline $\bar{G}$ & S & $618-000 G$ & 12522 & 1163 & 41 & 47683 & 0 & 0 & 0 \\
\hline $\bar{G}$ & S & $623-002 \mathrm{G}$ & 64 & 6 & 41 & 246 & 0 & 0 & 0 \\
\hline $\bar{G}$ & S & $623-027 \mathrm{G}$ & 288 & 27 & 9 & 243 & 0 & 0 & 0 \\
\hline $\bar{G}$ & S & $624-003 \mathrm{G}$ & 600 & 56 & 9 & 504 & 0 & 0 & 0 \\
\hline$\overline{\mathbf{G}}$ & $\mathbf{S}$ & $642-000 \mathrm{G}$ & 2800 & 260 & 9 & 2340 & $\overline{0}$ & 0 & 0 \\
\hline $\bar{G}$ & S & 643-000G & $\overline{0}$ & 0 & 0 & 0 & 1 & 0 & 1 \\
\hline $\bar{G}$ & s & 643-006G & 65 & 6 & 9 & 54 & $\overline{0}$ & 0 & 0 \\
\hline $\bar{G}$ & S & $643-012 \mathrm{G}$ & 720 & 67 & 9 & 603 & 0 & 0 & $\overline{0}$ \\
\hline $\bar{G}$ & S & $643-015 G$ & 192 & 18 & 9 & 162 & $\overline{0}$ & $\overline{0}$ & 0 \\
\hline G & $S$ & $643-016 \mathrm{G}$ & 240 & 22 & 9 & 198 & $\overline{0}$ & $\overline{0}$ & $\overline{0}$ \\
\hline G & $s$ & $643-017 \mathrm{G}$ & 160 & 15 & 11 & 165 & $\overline{0}$ & $\overline{0}$ & 0 \\
\hline $\bar{G}$ & $S$ & $643-018 \mathrm{G}$ & 160 & 15 & 9 & 135 & $\underline{0}$ & $\overline{0}$ & 0 \\
\hline$G$ & S & 643-020G & 360 & 33 & 9 & 297 & 0 & 0 & 0 \\
\hline$G$ & $S$ & 643-023G & 80 & 7 & 9 & 63 & 0 & 0 & 0 \\
\hline G & S & 643-029G & 3600 & 334 & 9 & 3006 & 0 & 0 & 0 \\
\hline$G$ & S & $643-030 \mathrm{G}$ & 8000 & 743 & 9 & 6687 & 0 & 0 & 0 \\
\hline G & s & $647-000 \mathrm{G}$ & 5000 & 465 & 9 & 4185 & 0 & 0 & 0 \\
\hline $\bar{G}$ & $\bar{s}$ & $647-0016$ & 1344 & 125 & 9 & 1125 & 0 & 0 & 0 \\
\hline $\bar{G}$ & S & $652-008 \mathrm{G}$ & $\overline{0}$ & 0 & 33 & 0 & 1 & 0 & 1 \\
\hline $\bar{G}$ & S & $661-000 \mathrm{G}$ & 2967 & 276 & 41 & 11316 & $\overline{0}$ & 0 & 0 \\
\hline G & is & $661-001 \mathrm{G}$ & 64 & 6 & 17 & 102 & $\overline{0}$ & 0 & 0 \\
\hline $\bar{G}$ & s & $661-002 \mathrm{G}$ & 1400 & 130 & 17 & 2210 & 0 & 0 & 0 \\
\hline $\bar{G}$ & is & $673-000 \mathrm{G}$ & $\overline{0}$ & 0 & 33 & 0 & 1 & 0 & 1 \\
\hline $\bar{G}$ & Is & $675-000 \mathrm{G}$ & $\overline{0}$ & 0 & 33 & 0 & $\overline{2}$ & $\overline{0}$ & $\overline{2}$ \\
\hline $\bar{G}$ & s & $677-000 \mathrm{G}$ & 0 & 0 & 33 & 0 & $\overline{4}$ & $\overline{0}$ & $\overline{4}$ \\
\hline$\overline{\mathrm{G}}$ & s & $678-000 \mathrm{G}$ & $\overline{0}$ & 0 & 33 & 0 & 1 & 0 & 1 \\
\hline $\bar{G}$ & ts & $681-001 \mathrm{G}$ & 12843 & 1193 & 37 & 44141 & 1 & 0 & 1 \\
\hline
\end{tabular}


Table A-18.--Incipient fire data for G,N,E Area

\begin{tabular}{|c|c|c|c|c|c|c|c|c|c|}
\hline \multirow[b]{2}{*}{ Area } & \multirow[b]{2}{*}{ Type } & \multirow[b]{2}{*}{ ID } & \multirow[b]{2}{*}{ Area, $\mathbf{f t}^{2}$} & \multirow[b]{2}{*}{ Area, $\mathrm{m}^{2}$} & \multirow{2}{*}{\begin{tabular}{|c|}
$\begin{array}{c}\text { years in } \\
\text { service to } \\
94\end{array}$ \\
\end{tabular}} & \multirow[b]{2}{*}{$y^{\prime *} \mathbf{n t}^{2}$} & \multicolumn{3}{|c|}{ Fire count } \\
\hline & & & & & & & $\begin{array}{c}\text { Original } \\
\text { count }\end{array}$ & '91 to '94 & $\begin{array}{l}\text { Sum of } \\
\text { all fires }\end{array}$ \\
\hline$G$ & $S$ & $681-003 \mathrm{G}$ & 12843 & 1193 & 37 & 44141 & 1 & 0 & 1 \\
\hline $\mathrm{G}$ & $S$ & $681-005 \mathrm{G}$ & 5899 & 548 & 37 & 20276 & 0 & 0 & 0 \\
\hline $\mathbf{G}$ & 5 & $681-006 \mathrm{G}$ & 2400 & 223 & 36 & 8028 & 0 & 0 & 0 \\
\hline$G$ & $S$ & $681-007 \mathrm{G}$ & 1790 & 166 & 39 & 6474 & 0 & 0 & 0 \\
\hline $\mathbf{G}$ & $S$ & $681-008 \mathrm{G}$ & 400 & 37 & 27 & 999 & of & 0 & 0 \\
\hline$G$ & $S$ & $681-009 \mathrm{G}$ & 480 & 45 & 9 & 405 & 0 & 0 & 0 \\
\hline$G$ & $S$ & $681-010 \mathrm{G}$ & 362 & 34 & 9 & 306 & 1 & 0 & 1 \\
\hline $\mathbf{G}$ & $S$ & $681-011 \mathrm{G}$ & 246 & 23 & 9 & 207 & 0 & 0 & 0 \\
\hline $\mathbf{G}$ & $S$ & $681-017 \mathrm{G}$ & & 0) & & 0 & 0 & 1 & 1 \\
\hline G & $\mathbf{S}$ & 686-003G & 720 & 67 & 16 & 1072 & 0 & 0 & 0 \\
\hline$G$ & $S$ & 690-000G & 11500 & 1068 & 9 & 9612 & 31 & 0 & 3 \\
\hline$G$ & $S$ & $701-002 G$ & 146 & 14 & 41 & 574 & 0 & 0 & 0 \\
\hline$G$ & $S$ & $701.003 G$ & 146 & 14 & 41 & 574 & 0 & 0 & 0 \\
\hline $\mathbf{G}$ & $S$ & $701-004 \mathrm{G}$ & 146 & 14 & 41 & 574 & of & 0 & 0 \\
\hline $\mathbf{G}$ & $S$ & $701-005 \mathrm{G}$ & 146 & 14 & 41 & 574 & of & 0 & 0 \\
\hline G & $S$ & $701-006 \mathrm{G}$ & 146 & 14 & 41 & 574 & 0 & 0 & 0 \\
\hline G & $S$ & $701-008 \mathrm{G}$ & 150 & 14 & 10 & 140 & 0 & 0 & 0 \\
\hline $\mathbf{G}$ & $S$ & $701.012 \mathrm{G}$ & 150 & 14 & 10 & 140 & 包 & 0 & 0 \\
\hline G & $\mathbf{S}$ & $701-013 \mathrm{G}$ & 150 & 14 & 10 & 140 & 0) & of & 0 \\
\hline G & $\mathbf{S}$ & $701-014 \mathrm{G}$ & 48 & 4 & 9 & 36 & 0 & of & 0 \\
\hline $\bar{G}$ & s & $701-015 \mathrm{G}$ & 48 & 4 & 9 & 36 & 0 & 0 & 0 \\
\hline $\mathbf{G}$ & $S$ & $701-016 \mathrm{G}$ & 48 & 4 & 9 & 36 & 0 & 0 & 0 \\
\hline $\mathrm{G}$ & $S$ & 703-005G & 2700 & 251 & 9 & 2259 & 0 & 의 & 0 \\
\hline$G$ & $S$ & $703-006 \mathrm{G}$ & 5600 & 520 & 9 & 4680 & 0 & 0 & 0 \\
\hline$G$ & S & $703-007 \mathrm{G}$ & 240 & 22 & 9 & 198 & 요 & 의 & 0 \\
\hline G & S & 703-009G & 200 & 19 & 9 & 171 & 의 & 0 & 0 \\
\hline$G$ & $S$ & $704-001 \mathrm{G}$ & 980 & 91 & 5 & 455 & 0 & 0 & 0 \\
\hline$G$ & $\mathbf{S}$ & $704-002 G$ & 784 & 73 & 5[ & 365 & 이 & 0] & 0 \\
\hline$G$ & S & 704-003G & 980 & 91 & 5 & 455 & 의 & 0 & 0 \\
\hline$G$ & $\mathrm{~S}$ & 704-004G & 460 & 43 & 5 & 215 & 0 & 0 & 0 \\
\hline$G$ & S & $705-000 \mathrm{G}$ & 11926 & 1108 & 5 & 5540 & 1 & 0 & 1 \\
\hline$G$ & $s$ & 706-000G & 7333 & 681 & 5 & 3405 & 0 & 0 & 0 \\
\hline $\mathbf{G}$ & $s$ & $706-001 \mathrm{G}$ & 200 & 19 & 5 & 95 & 0 & 0 & 0 \\
\hline $\bar{G}$ & $S$ & 706-003G & 10500 & 975 & 5 & 4875 & 0 & 0 & 0 \\
\hline $\mathbf{G}$ & S & $707-000 \mathrm{G}$ & 961 & 89 & 5 & 445 & 0 & 0 & 0 \\
\hline $\bar{G}$ & S & $709-000 \mathrm{G}$ & 10080 & 936 & 12 & 11232 & 0 & 0 & $\overline{0}$ \\
\hline$G$ & $S$ & $709-001 \mathrm{G}$ & 750 & 70 & 5 & 350 & 의 & 0 & $\overline{0}$ \\
\hline $\mathrm{G}$ & $s$ & $709-002 \mathrm{G}$ & 13550 & 1259 & 8 & 10072 & 0 & 0 & 0 \\
\hline $\bar{G}$ & $S$ & 709-004G & 11550 & 1073 & 6 & 6438 & 이 & 0 & $\underline{0}$ \\
\hline $\bar{G}$ & $S$ & $710-000 \mathrm{G}$ & 15360 & 1427 & 5) & 7135 & 1 & 0 & 1 \\
\hline $\mathrm{G}$ & $S$ & $710-002 G$ & 800 & 74 & 5 & 370 & 0 & 0 & $\underline{0}$ \\
\hline $\mathrm{G}$ & $S$ & $711.000 \mathrm{G}$ & 6000 & 557 & 5 & 2785 & 0 & 0 & $\underline{0}$ \\
\hline $\mathrm{G}$ & $S$ & $714-000 \mathrm{G}$ & 8000 & 743 & 37 & 27491 & 0 & 0 & $\overline{0}$ \\
\hline$G$ & $S$ & $714-002 \mathrm{G}$ & 11956 & 1111 & 37 & 4.1107 & 0 & 0 & $\underline{0}$ \\
\hline$G$ & $\mathbf{S}$ & 714-005G & 18670 & 1734 & $37 \mid$ & 64158 & 이 & 의 & 0 \\
\hline$G$ & $S$ & 714-006G & 16000 & 1486 & 37 & 54982 & 이 & 0 & 0 \\
\hline $\mathrm{G}$ & S & $714-007 \mathrm{G}$ & 16000 & 1486 & 37| & 54982 & 이 & 0 & 0 \\
\hline
\end{tabular}


Table A-18.--Incipient fire data for G,N,E Area

\begin{tabular}{|c|c|c|c|c|c|c|c|c|c|}
\hline Area & Type & D & Area, $\mathrm{ft}^{2}$ & Area, $\mathbf{m}^{2}$ & $\begin{array}{c}\text { years in } \\
\text { service to } \\
\text { '94 }\end{array}$ & yrs $^{*} \mathrm{~m}^{2}$ & $\begin{array}{c}\text { Origina } \\
\text { count }\end{array}$ & $\begin{array}{l}\text { Fire count } \\
\text { '91 to ' } 94\end{array}$ & $\begin{array}{l}\text { Sum of } \\
\text { all fires }\end{array}$ \\
\hline $\mathbf{G}$ & $S$ & $715-000 \mathrm{G}$ & 140 & 13 & 9 & 117 & 이 & 0 & 0 \\
\hline G & S & $715-001 \mathrm{G}$ & 576 & 54 & 9 & 486 & 0 & 0 & 0 \\
\hline G & IS & $717-000 \mathrm{G}$ & 3244 & 301 & 42 & 12642 & 0 & 1 & 1 \\
\hline $\mathrm{G}$ & 5 & $722-000 \mathrm{G}$ & 6030 & 560 & 9 & 5040 & o & 0 & 0 \\
\hline G & Is & $724-007 \mathrm{G}$ & 5231 & 486 & 9 & 4374 & 11 & 0 & 1 \\
\hline $\mathrm{G}$ & s & $724-008 \mathrm{G}$ & 1300 & 121 & 9 & 1089 & 0 & 0 & 0 \\
\hline G & S & $726-001 \mathrm{G}$ & 1600 & 149 & 9 & 1341 & 0 & 0 & 0 \\
\hline$G$ & $S$ & $728-000 \mathrm{G}$ & 1201 & 112 & 9 & 1008 & 0 & 0 & 0 \\
\hline$G$ & $\mathbf{S}$ & $730-000 \mathrm{G}$ & 8080 & 751 & 9 & 6759 & 0 & 0 & 0 \\
\hline G & $\mathbf{S}$ & $734-0000$ & 308 & 29 & 26 & 754 & 0 & 0 & $\overline{0}$ \\
\hline G & S & $735-007 \mathrm{G}$ & 1550 & 144 & 9 & 1296 & 0 & 0 & 0 \\
\hline$G$ & S & $735-007 \mathrm{G}$ & 4920 & 457 & 9 & 4113 & 0 & 0 & 0 \\
\hline G & S & $739-001 \mathrm{G}$ & 200 & 19 & 9 & 171 & 0 & 0 & 0 \\
\hline$G$ & s & $739-004 \mathrm{G}$ & 360 & 33 & 22 & 726 & 0 & 0 & 0 \\
\hline G & IS & $739-006 \mathrm{G}$ & 48 & 4 & 9 & 36 & 0 & 0 & 0 \\
\hline $\mathbf{G}$ & IS & $741-000 \mathrm{G}$ & 600 & 56 & 9 & 504 & 0 & 1 & 1 \\
\hline G & S & $741-001 \mathrm{G}$ & 400 & 37 & 9 & 333 & 0 & 0 & 0 \\
\hline $\mathrm{G}$ & S & $741-002 \mathrm{G}$ & 8000 & 743 & 9 & 6687 & 0 & 0 & 0 \\
\hline $\mathrm{G}$ & S & $760-000 G$ & 4074 & 378 & 38 & 14364 & 0 & 0 & 0 \\
\hline$G$ & Is & $760-001 \mathrm{G}$ & 4138 & 384 & 38 & 14592 & 1 & 0 & 1 \\
\hline $\mathrm{G}$ & S & $760-002 G$ & 714 & 66 & 38 & 2508 & 의 & 0 & 0 \\
\hline G & $S$ & $760-003 G$ & 3024 & 281 & 38 & 10678 & 0 & 0 & 0 \\
\hline G & s & $760-004 \mathrm{G}$ & 3760 & 349 & 21 & 7329 & 0 & 0 & 0 \\
\hline G & S & $760-005 \mathrm{G}$ & 1792 & 166 & 5 & 830 & 0 & 0 & 0 \\
\hline G & $S$ & $760-008 \mathrm{G}$ & 865 & 80 & 15 & 1200 & 0 & 0 & 0 \\
\hline G & S & $760-009 \mathrm{G}$ & 3600 & 334 & 13 & 4342 & 0 & 0 & 0 \\
\hline G & S & $760-010 \mathrm{G}$ & 144 & 13 & 13 & 169 & 0. & 0 & 0 \\
\hline$G$ & S & $760-011 \mathrm{G}$ & 11401 & 1059 & 12 & 12708 & 1 & 0 & 1 \\
\hline G & IS & $760-012 G$ & 1500 & 139 & 11 & 1529 & 0 & 0 & 0 \\
\hline G & IS & $760-013 G$ & 2000 & 186 & 6 & 1116 & 0 & 0 & 0 \\
\hline G & IS & $772-000 \mathrm{G}$ & 7695 & 715 & 38 & 27170 & 0 & 0 & 0 \\
\hline G & S & $772-001 \mathrm{G}$ & 7786 & 723 & 38 & 27474 & 0 & 0 & 0 \\
\hline G & IS & $772-002 \mathrm{G}$ & 392 & 36 & 34 & 1224 & 1 & 0 & 1 \\
\hline$G$ & s & $772-003 G$ & 240 & 22 & 31 & 682 & 의 & 0 & 0 \\
\hline $\mathbf{G}$ & Is & $772-005 G$ & 668 & 62 & 30 & 1860 & 0 & 0 & 0 \\
\hline G & $\mathbf{S}$ & $772-007 \mathrm{G}$ & 8000 & 743 & 41 & 30463 & 0 & 0 & 0 \\
\hline $\mathbf{G}$ & IS & $772-008 G$ & 7800 & 725 & 26 & 18850 & 0 & 0 & 0 \\
\hline G & IS & $772-009 \mathrm{G}$ & 7800 & 725 & 26 & 18850 & 0 & 0 & 0 \\
\hline G & Is & $772-010 \mathrm{G}$ & 7800 & 725 & 26 & 18850 & 0 & 0 & 0 \\
\hline G & IS & $772-013 G$ & 720 & 67 & 24 & 1608 & 0 & 0 & 0 \\
\hline G & IS & $772-014 \mathrm{G}$ & 672 & 62 & 20 & 1240 & 0 & 0 & 0 \\
\hline$G$ & $S$ & $772-017 \mathrm{G}$ & 768 & 71 & 19 & 1349 & 0) & 0 & 0 \\
\hline G & IS & $772-018 \mathrm{G}$ & 660 & 61 & 9 & 549 & 0 & 0 & 0 \\
\hline G & S & $772-019 \mathrm{G}$ & 660 & 61 & 9 & 549 & 0 & 0 & 0 \\
\hline G & IS & $772-020 \mathrm{G}$ & 1000 & 93 & 19 & 1767 & 1 & 0 & 1 \\
\hline G & S & $772-021 \mathrm{G}$ & 648 & 60 & 9 & 540 & 0 & 0 & 0 \\
\hline G & IS & $772-022 G$ & 648 & 60 & 9 & 540 & 0 & 0 & 0 \\
\hline
\end{tabular}


Table A-18.--Incipient fire data for G,N,E Area

\begin{tabular}{|c|c|c|c|c|c|c|c|c|c|}
\hline \multirow[b]{2}{*}{ Area } & \multirow[b]{2}{*}{ Type } & \multirow[b]{2}{*}{ ID } & \multirow[b]{2}{*}{ Area, $\boldsymbol{f}^{2}$} & \multirow[b]{2}{*}{ Area, $\mathbf{m}^{2}$} & \multirow{2}{*}{\begin{tabular}{|c|} 
years in \\
service to \\
'94
\end{tabular}} & \multirow[b]{2}{*}{$y r{ }^{*} m^{2}$} & \multicolumn{3}{|c|}{ Fire count } \\
\hline & & & & & & & $\begin{array}{l}\text { Original } \\
\text { count }\end{array}$ & '91 to '94| & $\begin{array}{l}\text { Sum of } \\
\text { all fires }\end{array}$ \\
\hline $\mathbf{G}$ & $s$ & $772.025 \mathrm{G}$ & 2400 & 223 & 9 & 2007 & 0 & 1 & 1 \\
\hline G & s & $772-026 \mathrm{G}$ & 200 & 19 & 12 & 228 & 0 & 0 & 0 \\
\hline G & S & $904-116 \mathrm{G}$ & 48 & 4 & 7 & 28 & 0 & 0 & 0 \\
\hline $\mathbf{G}$ & S & $905-006 \mathrm{G}$ & 48 & 4 & 41 & 164 & 0 & 0 & 0 \\
\hline $\bar{G}$ & $S$ & $905-007 \mathrm{G}$ & 48 & 4 & 41 & 164 & $\overline{0}$ & 0 & 0 \\
\hline G & $S$ & $905-008 \mathrm{G}$ & 48 & 4 & 41 & 164 & 0 & 0 & 0 \\
\hline G & S & $905-010 \mathrm{G}$ & 48 & 4 & $41]$ & 164 & 0 & 이 & 0 \\
\hline G & S & $905-011 \mathrm{G}$ & 36 & 3 & 41 & 123 & 0 & 0 & 0 \\
\hline G & $S$ & $905-074 \mathrm{G}$ & 36 & 3 & 20 & 60 & 0 & 0 & 0 \\
\hline G & $S$ & $905-109 \mathrm{G}$ & 48 & 4 & 8 & 32 & 0 & 0 & 0 \\
\hline G & $S$ & $905-110 \mathrm{G}$ & 48 & 4 & 8 & 32 & 0 & 0 & 0 \\
\hline G & $\mathbf{S}$ & $905-111 \mathrm{G}$ & 48 & 4 & 8 & 32 & 0 & 0 & 0 \\
\hline$G$ & $S$ & $905-115 \mathrm{G}$ & 48 & 4 & 7 & 28 & 0 & 0 & 0 \\
\hline $\mathbf{N}$ & S & $704-003 N$ & 12000 & 1115 & 11 & $1: 265$ & 0 & 0 & 0 \\
\hline $\mathbf{N}$ & $\mathbf{S}$ & $711-000 \mathrm{~N}$ & 45600 & 4236 & 37 & 156732 & $\underline{0}$ & 2 & 2 \\
\hline $\mathbf{N}$ & S & $711-002 \mathrm{~N}$ & & 이 & & 0 & 0 & 1 & 1 \\
\hline $\mathbf{N}$ & S & $713-000 \mathrm{~N}$ & 30000 & 2787 & 37 & 103119 & $\underline{0}$ & 0 & 0 \\
\hline $\mathbf{N}$ & S & $713-001 \mathrm{~N}$ & 30000 & 2787 & 12 & 3.3444 & 0 & 0 & 0 \\
\hline $\mathbf{N}$ & S & $716-000 \mathrm{~N}$ & & 0 & & 0 & 0 & 1 & 1 \\
\hline $\mathbf{N}$ & $\mathbf{S}$ & $716-000 \mathrm{~N}$ & 15850 & 1473 & 37 & 54501 & 0 & 0 & 0 \\
\hline $\mathbf{N}$ & S & $716-005 \mathrm{~N}$ & & 0 & & 0 & 0 & 1 & 1 \\
\hline $\mathbf{N}$ & $S$ & $717-005 \mathrm{~N}$ & 22000 & 2044 & 37 & 7,5628 & 0 & 0 & 0 \\
\hline $\mathbf{N}$ & $\mathbf{S}$ & $717-008 \mathrm{~N}$ & 7400 & 687| & 37 & 25419 & $\underline{0}$ & 0 & 0 \\
\hline $\mathbf{N}$ & $\mathbf{S}$ & $717-010 \mathrm{~N}$ & 12000 & 1115 & 15 & 15725 & 2 & 0 & 2 \\
\hline $\mathbf{N}$ & $S$ & $717-011 \mathrm{~N}$ & 5265 & 489 & 37 & 13093 & 0 & 0 & $\overline{0}$ \\
\hline $\mathbf{N}$ & $S$ & $719-000 \mathrm{~N}$ & 6500 & 604 & 37 & 22348 & 0 & 0 & 0 \\
\hline $\mathbf{N}$ & $S$ & $725-001 \mathrm{~N}$ & 4500 & 418 & 12 & 5016 & 0 & 0 & 0 \\
\hline $\mathbf{N}$ & $s$ & $728-012 \mathrm{~N}$ & 의 & 의 & 0 & 0 & $\underline{0}$ & 1 & 1 \\
\hline $\mathbf{N}$ & S & $731-000 \mathrm{~N}$ & 14000 & 1301 & 9 & 11709 & 0 & 1 & 1 \\
\hline $\mathbf{N}$ & s & $741-000 \mathrm{~N}$ & 0 & 이 & 0 & 0 & 0 & 1 & 1 \\
\hline
\end{tabular}


Appendix B, SRS Fire Loss Data for 1991, 1992, 1993 and 1994 
WSRC-TR-99-00361

This page intentionally blank. 
Table B-1.--Incipient fires occuring between 1991 and 1994

\begin{tabular}{|c|c|c|c|c|c|}
\hline Building & 1991 & 1992 & 1993 & 1994 & $\begin{array}{c}\text { Sum of fires } \\
\text { between } 91.94\end{array}$ \\
\hline \multicolumn{6}{|l|}{$703-000 \mathrm{~A}$} \\
\hline \multicolumn{6}{|l|}{$703-041 \mathrm{~A}$} \\
\hline \multicolumn{6}{|l|}{$703.042 \mathrm{~A}$} \\
\hline \multicolumn{6}{|l|}{$715-000 \mathrm{~A}$} \\
\hline \multicolumn{6}{|l|}{$\frac{716-000 \mathrm{~A}}{716-000 \mathrm{~A}}$} \\
\hline \multirow{2}{*}{\multicolumn{6}{|c|}{$716-002 \mathrm{~A}$}} \\
\hline \multicolumn{2}{|l|}{$719-011 \mathrm{~A}$} & & & & \\
\hline \multirow{2}{*}{\multicolumn{6}{|c|}{$\frac{723-000 \mathrm{~A}}{735-002 \mathrm{~A}}$}} \\
\hline & & & & & \\
\hline \multicolumn{6}{|l|}{$777-010 \mathrm{~A}$} \\
\hline \multicolumn{6}{|l|}{$778-005 \mathrm{~A}$} \\
\hline \multirow{2}{*}{\multicolumn{6}{|c|}{$\frac{784-000 \mathrm{~A}}{789-000 \mathrm{~A}}$}} \\
\hline & & & & & \\
\hline \multirow{2}{*}{\multicolumn{6}{|c|}{$\begin{array}{r}\mathrm{A} \\
703-000 \mathrm{~B} \\
\end{array}$}} \\
\hline & & & & & \\
\hline \multicolumn{6}{|l|}{$703-001 \mathrm{~B}$} \\
\hline \multicolumn{6}{|l|}{ B } \\
\hline \multirow{2}{*}{\multicolumn{6}{|c|}{$704-006 \mathrm{C}$}} \\
\hline & & \multicolumn{4}{|c|}{$706-000 \mathrm{C}$} \\
\hline \multirow{2}{*}{\multicolumn{6}{|c|}{$\frac{706-009 \mathrm{C}}{700 \mathrm{C}}$}} \\
\hline & & & & & \\
\hline \multicolumn{6}{|l|}{$701-001 F$} \\
\hline \multicolumn{6}{|l|}{$772.000 \mathrm{~F}$} \\
\hline 7005 & & & & & \\
\hline \multicolumn{6}{|l|}{$703-000 \mathrm{H}$} \\
\hline \multicolumn{6}{|l|}{$704-000 \mathrm{H}$} \\
\hline \multirow{2}{*}{\multicolumn{6}{|c|}{$\frac{705-000 \mathrm{H}}{700 \mathrm{H}}$}} \\
\hline & & & & & \\
\hline \multirow{2}{*}{\multicolumn{6}{|c|}{$704-022 \mathrm{~K}$}} \\
\hline & & & & & \\
\hline \multicolumn{6}{|l|}{$700 \mathrm{~K}$} \\
\hline \multicolumn{6}{|l|}{$105-000 \mathrm{C}$} \\
\hline \multicolumn{6}{|l|}{$105-000 \mathrm{~K}$} \\
\hline $183-004 \mathrm{~K}$ & & & & & \\
\hline $105-000 \mathrm{P}$ & & & & & \\
\hline 100 & & & 7 & & 1. \\
\hline 484-000D & & & & & 1 \\
\hline 4000 & & & & & $\mathbf{1}$ \\
\hline
\end{tabular}




\begin{tabular}{|c|c|c|c|c|c|}
\hline Building & 1991 & 1992 & 1993 & 1994 & $\begin{array}{c}\text { Sum of tires } \\
\text { between } 91-94\end{array}$ \\
\hline $221-000 \mathrm{~F}$ & 2 & 1 & 1 & 21 & 6 \\
\hline $221-001 F$ & & & 1 & & 1 \\
\hline $221-025 \mathrm{~F}$ & 1 & & & & 1 \\
\hline $241-028 \mathrm{~F}$ & & & 1 & & $\sqrt[1]{1}$ \\
\hline $241-064 \mathrm{~F}$ & & & 1 & & 1 \\
\hline $247-000 \mathrm{~F}$ & & & & 1 & 1 \\
\hline $292-000 \mathrm{~F}$ & & 2 & & & 2 \\
\hline $200 F$ & 4 & 3 & 4 & 3 & 14 \\
\hline $218-000 \mathrm{H}$ & & & $\square$ & 11 & 1 \\
\hline $221-000 \mathrm{H}$ & & 2 & 2 & 2 & 6 \\
\hline $234-000 \mathrm{H}$ & & & 1 & & 1 \\
\hline $241-000 \mathrm{H}$ & 1 & & & & 1 \\
\hline $241.082 \mathrm{H}$ & & 1 & & & 1 \\
\hline $242-025 \mathrm{H}$ & & & & 1 & 1 \\
\hline $254-005 H$ & & & 1 & & 1 \\
\hline $284-000 \mathrm{H}$ & & & & & 1 \\
\hline $294-000 \mathrm{H}$ & & & 1 & & 1 \\
\hline $299-000 \mathrm{H}$ & & & 1 & & 1 \\
\hline $200 \mathrm{H}$ & 2 & 3 & 6 & 4 & 15 \\
\hline $305-001 M$ & & & 1 & & 1 \\
\hline $320-000 \mathrm{M}$ & 1 & 1 & & 1 & 3 \\
\hline $321-000 \mathrm{M}$ & & 1 & & & 1 \\
\hline $300 M$ & 1 & 2 & 1 & 1 & 5 \\
\hline $210-000 \mathrm{~s}$ & & & 1 & & 1 \\
\hline $221-000 s$ & & & 1 & 1 & 2 \\
\hline $704-000 s$ & & & & 1 & 1 \\
\hline 704-025S & & & & 1 & 1 \\
\hline $704-0715$ & & & & 1 & 1 \\
\hline $\mathbf{S}$ & $\overline{0}$ & 0 & 2 & 4 & 6 \\
\hline 643-000E & & 1 & & 1 & 2 \\
\hline $681-017 \mathrm{G}$ & 1 & & & & 1 \\
\hline $717-000 \mathrm{G}$ & & & & 1 & 1 \\
\hline $741-000 \mathrm{G}$ & & & & 1 & 1 \\
\hline $772-025 \mathrm{G}$ & 1 & & & & 1 \\
\hline $711-000 \mathrm{~N}$ & $\overline{2}$ & & & & $\overline{2}$ \\
\hline $711-002 \mathrm{~N}$ & & & & 1 & 1 \\
\hline $716-000 \mathrm{~N}$ & & & 1 & & 1 \\
\hline 716-005N & 1 & & & & 1 \\
\hline $728-012 N$ & & 1 & & & 1 \\
\hline $731-000 \mathrm{~N}$ & & & & 1 & 1 \\
\hline $741-000 \mathrm{~N}$ & & & 1 & & 1 \\
\hline E/G/N & 5 & 2 & 2 & 5 & 14 \\
\hline Total fires & 26 & 23 & 35 & 22 & 106 \\
\hline
\end{tabular}


Appendix C, Background Material for Original Fire Frequency Estimator 
WSRC-TR-99-00361

This page intentionally blank:. 


\section{DEVELOPMENT OF BUILDING FIRE FREQUENCIES FOR THE MAU REPORT}

The initial MAU report, WSRC-TR-91-469, and the draft final report, WSRC-TR-91-424, developed fire frequencies for the various buildings under consideration for a fire protection upgrade by estimating a site severe fire frequency per 10,000 square feet of floor space, assigning multipliers to the different areas based on history of fires, and assigning multipliers based on usage of the buildings. The site severe fire frequency was developed from WSRCRP-90-143, "Analysis of SRS Industrial Fire Records".

The site severe fire frequency ( $F_{\text {sverute }}$ ) was estimated as the total site fire frequency ( $F_{\text {tomite }}$ ) multiplied by the expected occurrence of a fire of sufficient magnitude to cause substantial damage (deaths of a fraction of the occupants, or property damage losses on the order of $5 \%$ to $70 \%$ of the buildings replacement value which varies from about $\$ 20$ million upward to $\$ 800$ million). $F_{\text {toretein }}=$ $10,000 * N_{100} /\left(A_{100} * Y\right)=40.5 \mathrm{E}-03$ fires per $10,000 \mathrm{sq}$ ft-yr where $N_{100}$ is the total number of fires observed in facilities with $A_{a x} \mathrm{sq} f t$ of floor space over a period of $Y$ years. At the time of the report there had been 0 fires out of 669 fires reported that caused the magnitude of damage stated above, therefore it was assumed that one occurs every 669 fires to yield a site severe fire frequency of $F_{\text {everesice }}=40.5 \mathrm{E}-03 *(1 / 669)=60.5 \mathrm{E}-06$ per $10,000 \mathrm{sq}$ ft per $\mathrm{yr}$.

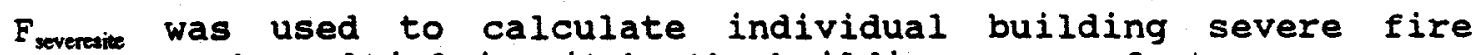
frequency by multiplying it by the building square footage, an area specific multiplier, and a usage dependent multiplier. The area specific multiplier weighted the fire frequency depending on the area that the building was in (based on history of fires in the various areas), and the usage dependent multiplier weighted the fire frequency depending on the activity that was performed in the building (manufacturing, chemical storage, offices, etc.)

The effort in this report is to use the building/area fire data used in WSRC-RP-90-143 to develop building specific severe fire frequencies or area specific fire frequencies based on actual occurrence of fires in the buildings/areas. This will reduce uncertainties about the data used in the initial and final draft versions of the MAU report.

\section{METHODS}

The data base used to develop WSRC-RP-90-143 was obtained from M.D. 
Brandyberry. The data base listed all fires that were reported during the period January 1958 through December 1990, and it listed the building that the fire occurred in. There were a total of 710 facility fires reported during the periocl. The data base was sorted by area and building to facilitate extraction of numbers of fires per building and numbers of fires per area. For the buildings in the MAU analysis, the number of fires reported if any, were gathered and placed in a spreadsheet along with the associated buildings (see Table 1 , columns B \& C). Columns D \& $E$ of Table 1 show the month and year that the buildings were completed according to property accounting records (if available), and column $F$ calculates the years that the buildings have been in service during the period $1 / 58$ through $12 / 90$. When property accounting records were not available for certain buildings, cognizant personnel provided an estimate of start-up date for some of the facilities. These estimates are shown in column $F$ with no entries to columns $C$ or D. The building specific severe fire frequency (Table 1, column $G)$ is calculated by the following formula:

$F_{\text {bideppecific }}=$ \# Fires Reported / \# Yrs in Servicie $1 / 58-12 / 90 *(1 / 710)$

This was considered to be a statistically sufficient value to use in the MAU analysis if; 1) there were 2 or more fires reported in the facility, or 2) the \# yrs in service was judged to be sufficient to calculate a frequency based on one reported fire.

If either of the two conditions listed above were not met, an area specific fire frequency per 10,000 square : :eet/year was developed from Brandyberry's data base to reduce uricertainties associated with using a site severe fire frequency with weighting factors. Table 2 shows by area(from property accounting records), the buildings within the area, the square footacje of the buildings, and the number of fires reported for the buildings. At the end of each area, the total square footage is shown, and the total number of reported fires is shown. From these values, we can derive an area specific fire frequency per 10,000 square: feet per year by the following formula:

$F_{\text {ereapecific }}=F_{\text {lowal }} * 10,000 /\left(A_{\text {tox }} * 710\right.$ fires $* 33$ yrs $)$

where: $F_{\text {tow }}=$ Total number of fires reported for the area, $A_{\text {tom }}=$ Total square footage of the buildings in the area 33 yrs is the assumed \# years of service for the area $(1 / 58-12 / 90)$

The area specific data was broken down into areas that would contain like buildings, i.e. manufacturing or office, in order to 
eliminate usage of weighting factors. The following are the areas in which an area specific severe fire frequency was developed, and the actual area specific fire frequency is also listed.

\begin{tabular}{|c|c|}
\hline Area & $\begin{array}{l}\text { Severe Fire Fr } \\
\text { (per } 10,000 \mathrm{sq}\end{array}$ \\
\hline $\begin{array}{l}100 \\
200 \mathrm{~F} \\
200 \mathrm{H} \\
\mathrm{A} \\
700 \mathrm{~F} \\
700 \mathrm{H} \\
300 \mathrm{M} \\
400 \mathrm{D} \\
\mathrm{B} \\
700 \mathrm{C} \\
700 \mathrm{D} \\
\text { G/CS } \\
\text { TNX } \\
700 \mathrm{~K} \\
700 \mathrm{~L} \\
700 \mathrm{M} \\
700 \mathrm{P} \\
700 \mathrm{R}\end{array}$ & $\begin{array}{l}1.21 E-05 \\
6.61 E-05 \\
3.97 E-05 \\
2.41 E-05 \\
6.47 E-05 \\
2.28 E-05 \\
1.03 E-04 \\
8.67 E-05 \\
1.74 E-05 \\
1.66 E-05 \\
4.08 E-05 \\
1.84 E-05 \\
1.67 E-05 \\
9.05 E-06 \\
8.64 E-05 \\
7.67 E-05 \\
5.24 E-05 \\
2.04 E-05\end{array}$ \\
\hline
\end{tabular}

There was not enough data to calculate an area specific severe fire frequency for S-Area, so it was assumed to be the average of $200-F$ and $200-\mathrm{H}$.

This data was used in column I of Table 1 to calculate building severe fire frequency for those building that had insufficient data to determine a building specific severe fire frequency by multiplying the area specific severe fire frequency per 10,000 square feet by the square footage of the building and dividing the result by 10,000 . Therefore, column $\mathrm{J}$ of Table 1 contains the recommended values for building severe fire frequencies, where the values in bold represent a frequency based on actual fires in the building, and the values that are not in bold represent a frequency based on actual fires in the area.

As a final "sanity" check of the building severe fire frequencies, the number of small fires that were expected to have occurred over the existence of the building was back calculated using the building severe fire frequency from column $J$, and the number of years in service (during the period 1/58-12/90) of the building from column F. If the expected number of fires came out greater than 1 for the buildings that had actually experienced 0 fires, then the building severe fire frequency was forced to a value that would be consistent with the building experiencing 1 fire over it's existence. This situation did occur with 6 buildings; 305-A, 
$719-\mathrm{A}, 483-1 \mathrm{D}, 717-\mathrm{F}, 711-\mathrm{N}$, and 713-N. The forced values are highlighted in the Table as large, bold, italic numbers.

\section{LIMITATIONS}

The limitations of using this data in the MAlJ prioritization effort are concerned with uncertainties in the reporting of fires and therefore the fire database, and uncertainties in area square footage. As acknowledged in WSRC-RP-90-143, the industrial fire data base is not complete because undoubtedly, there have been fires that have occurred that were not reposted. This uncertainty was also present in the earlier MAU reports since it relied on the data to determine area specific multipliers.

The square footage values used in Table 2 came from the property accounting group in SRL. Discrepancies have been shown in this data because it was initially used as the raw data for square footage of the buildings considered in the MAU analysis, and when cognizant personnel reviewed the input data there were some gross errors uncovered with some square footage values. These errors were corrected for those buildings. The other buildings that were not part of the MAU analysis but were used in the development of area specific fire frequency development could be suspect. 
Table 1

\begin{tabular}{|c|c|c|c|c|}
\hline $\mathbf{G}$ & $\bar{H}$ & 1 & $J$ & $\bar{K}$ \\
\hline BLDG & & AREA SPECIFIC & ADOPTED VALUE & \# OF FIRES \\
\hline SPECIFIC & & SEVERE & FOR BLDG & EXPECTED \\
\hline SEVERE & BLDG & FIRE FREO I & SEVERE & BASED ON \\
\hline FIRE FREQ & SOFT & $10.000 \mathrm{SQ} \mathrm{FT}$ & FIRE FREQ & BLDG SFREO \\
\hline $4.27 E-05$ & 18606 & 2.41E-05 & $4.27 E-05$ & 1.00 \\
\hline \multirow[t]{3}{*}{$8.54 E-05$} & 5195 & 2.41E-05 & $8.54 E-05$ & 2.00 \\
\hline & 20000 & $2.41 E-05$ & $4.82 E-05$ & .21 \\
\hline & 20000 & $2.41 \mathrm{E}-05$ & $4.82 E-05$ & .23 \\
\hline \multirow[t]{2}{*}{$9.80 E-04$} & 20000 & 2.41E-05 & $9.80 E-04$ & 4.00 \\
\hline & 20000 & $2.41 \mathrm{E}-05$ & $4.82 E-05$ & .18 \\
\hline 2.99E-04 & 177000 & 2.41E-05 & $2.99 E-04$ & 7.00 \\
\hline 2.99E-04 & 40068 & $2.41 E-05$ & $2.99 E-04$ & 7.00 \\
\hline \multirow[t]{4}{*}{$4.27 E-05$} & 26487 & 2.41E-05 & $4.27 E-05$ & 1.00 \\
\hline & 6900 & 2.41E-05 & $1.66 \mathrm{E}-05$ & .11 \\
\hline & 7800 & 2.41E-05 & $1.88 \mathrm{E}-05$ & .09 \\
\hline & 11700 & 2.41E-05 & $2.82 \mathrm{E}-05$ & .66 \\
\hline \multirow[t]{5}{*}{$2.86 E-04$} & 20000 & $2.41 E-05$ & $4.82 E-05$ & .17 \\
\hline & 1800 & 2.41E-05 & $4.34 E-06$ & .05 \\
\hline & 7500 & 2.41E-05 & $1.81 E-05$ & .09 \\
\hline & 10286 & 2.41E-05 & $2.48 \mathrm{E}-05$ & .58 \\
\hline & 29000 & $2.41 E-05$ & 6.99E-05 & .36 \\
\hline \multirow[t]{2}{*}{ 4.27E-05 } & 4450 & 2.41E-05 & $4.27 \mathrm{E}-05$ & 1.00 \\
\hline & 20000 & $2.41 E-05$ & $4.82 \mathrm{E}-05$ & .21 \\
\hline $4.10 \mathrm{E}-03$ & 304000 & $2.41 \mathrm{E}-05$ & $4.10 \mathrm{E}-03$ & 96.00 \\
\hline $4.27 E-05$ & 2501 & 2.41E-05 & $4.27 E-05$ & 1.00 \\
\hline $8.54 E-05$ & 6000 & 2.41E-05 & $8.54 E-05$ & 2.00 \\
\hline $8.54 E-05$ & 6000 & $2.41 \mathrm{E}-05$ & $8.54 E-05$ & 2.00 \\
\hline $3.67 E-04$ & 20000 & $1.74 \mathrm{E}-05$ & $3.48 \mathrm{E}-05$ & .09 \\
\hline $3.67 E-04$ & 20000 & $1.74 E-05$ & $3.48 E-05$ & .09 \\
\hline $7.26 E-04$ & 500000 & $1.21 \mathrm{E}-05$ & $7.26 \mathrm{E}-04$ & 17.00 \\
\hline \multirow[t]{3}{*}{$7.26 E-04$} & 500000 & $1.21 \mathrm{E}-05$ & $7.26 \mathrm{E}-04$ & 17.00 \\
\hline & 6000 & $1.21 E-05$ & $7.26 \mathrm{E}-06$ & 17 \\
\hline & 6000 & $1.21 \mathrm{E}-05$ & $7.26 \mathrm{E}-06$ & .17 \\
\hline
\end{tabular}


Table 1

\begin{tabular}{|c|c|c|c|c|c|c|c|c|c|c|c|}
\hline & A & B & c & $\mathbf{D}$ & $\mathbf{E}$ & $F$ & $\mathbf{G}$ & $H$ & 1 & $\mathbf{J}$ & $\mathbf{K}$ \\
\hline 1 & & & & & & & BLDG & & AREA SPECIFIC & ADOPTED VALUE & \# OF FIRES \\
\hline 2 & & & \# FIRES & & & YAS IN & SPECIFIC & & SEVERE & FOR BLDG & EXPECTED \\
\hline 3 & & & REPORTED & MTH & YR & SERVICE & SEVERE & BLDG & FIRE FREQ I & SEVERE & BASED ON \\
\hline 4 & AREA & BLDG & $1 / 58-12 / 90$ & COMP & COMP & $1 / 58-12 / 90$ & FIRE FREO & SOFT & $10,000 \mathrm{Sa} \mathrm{FT}$ & FIRE FREQ & BLDG SFREQ \\
\hline 5 & & & & & & & & & & & \\
\hline 35 & C & $151-1 \mathrm{C}$ & 의 & 4 & 55 & 33.00 & & 6400 & $1.21 \mathrm{E}-05$ & $7.74 \mathrm{E}-06$ & .18 \\
\hline 36 & C & $151-2 \mathrm{C}$ & of & 4 & 55 & 33.00 & & 6400 & 1.21E-05 & $7.74 \mathrm{E}-06$ & .18 \\
\hline 37 & C & $190-\mathrm{C}$ & of & 4 & 55 & 33.00 & & 12000 & $1.21 \mathrm{E}-05$ & $1.45 \mathrm{E}-05$ & .34 \\
\hline 38 & C & $707-C$ & 0 & 6 & 88 & 2.58 & & 44728 & $1.66 \mathrm{E}-05$ & 7.42E-05 & .14 \\
\hline 39 & D & 483-1D & 1 & 2 & 53 & 33.00 & 4.27E-05 & 38000 & 8.67E-05 & 4.27E-05 & 1.00 \\
\hline 40 & D & 484-D(1) & 24 & 2 & 53 & 33.00 & $1.02 E-03$ & 250000 & 8.67E-05 & $1.02 \mathrm{E}-03$ & 24.00 \\
\hline 41 & D & 484-D (2) & 24 & 2 & 53 & 33.00 & $1.02 E-03$ & 250000 & $8.67 \mathrm{E}-05$ & $1.02 E-03$ & 24.00 \\
\hline 42 & D & 485-D & 0 & 2 & 53 & 33.00 & & 3826 & 8.67E-05 & $3.32 \mathrm{E}-05$ & .78 \\
\hline 43 & $F$ & $211-1 \mathrm{~F}(1)$ & 0 & 10 & 85 & 5.25 & & 6000 & 6.61E-05 & $3.97 \mathrm{E}-05$ & .15 \\
\hline 44 & $\bar{F}$ & $211-1 F(2)$ & of & 10 & 85 & 5.25 & & 6000 & 6.61E-05 & 3.97E-05 & .15 \\
\hline 45 & $F$ & $211 \cdot F(1)$ & 3 & 9 & 54 & 33.00 & $1.28 \mathrm{E}-04$ & 10000 & $6.61 \mathrm{E}-05$ & $1.28 E-04$ & 3.00 \\
\hline 46 & $\mathbf{F}$ & $211-F(2)$ & 3 & 9 & 54 & 33.00 & $1.28 E-04$ & 10000 & 6.61E-05 & $1.28 E-04$ & 3.00 \\
\hline 47 & $\mathbf{F}$ & 221-1f (1) & 1 & 9 & 54 & 33.00 & $4.27 \mathrm{E}-05$ & 24067 & 6.61E-05 & $4.27 E-05$ & 1.00 \\
\hline 48 & $F$ & $221-1 F(2)$ & 1 & 9 & 54 & 33.00 & 4.27E-05 & 24067 & 6.61E-05 & 4.27E-05 & 1.00 \\
\hline 49 & $\bar{F}$ & $221-F(1)$ & 74 & 9 & 54 & 33.00 & $3.16 \mathrm{E}-03$ & 329600 & 6.61E-05 & 3.16E-03 & 74.00 \\
\hline 50 & $F$ & $221-F(2)$ & 74 & 9 & 54 & 33.00 & $3.16 \mathrm{E}-0 \mathrm{~S}$ & š̄ & ô.0ic-0ิ5 & $3.965-43$ & 74.00 \\
\hline 51 & $F$ & $222 \cdot F$ & 1 & 6 & 58 & 32.58 & 4.32E-05 & 5989 & 6.61E-05 & 4.32E-05 & 1.00 \\
\hline 52 & $\mathbf{F}$ & $235-F$ & 5 & 9 & 54 & 33.00 & 2.13E-04 & 47080 & 6.61E-05 & $2.13 E-04$ & 5.00 \\
\hline 53 & $F$ & $247-F$ & 4 & 7 & 88 & 2.50 & $2.25 E-03$ & 140000 & 6.61E-05 & 2.25E-03 & 4.00 \\
\hline 64 & $F$ & $251-F$ & 1 & 9 & 54 & 33.00 & 4.27E-05 & 1923 & 6.61E-05 & $4.27 E-05$ & 1.00 \\
\hline 55 & $\boldsymbol{F}$ & 284-8F & of & 11 & 85 & 5.17 & & 2826 & 6.61E-05 & 1.87E-05 & .07 \\
\hline 56 & $F$ & 292-2F (1) & of & 11 & 89 & 1.17 & & 3470 & 6.61E-05 & 2.29E-05 & .02 \\
\hline 57 & $\mathbf{F}$ & $292.2 F(2)$ & 0 & 11 & 89 & 1.17 & & 3470 & 6.61E-05 & 2.29E-05 & .02 \\
\hline 58 & $\mathbf{F}$ & 292-F & 5 & 9 & 54 & 33.00 & $2.13 E-04$ & 12482 & 6.61E-05 & $2.13 E-04$ & 5.00 \\
\hline 59 & $F$ & $703-F$ & 1 & 9 & 84 & 6.33 & 2.22E-04 & 25000 & $6.47 \mathrm{E}-05$ & 2.22E-04 & 1.00 \\
\hline 60 & $\mathbf{F}$ & 717-F & 1 & 9 & 54 & 33.00 & 4.27E-05 & 52500 & 6.47E-05 & 4.27E-05 & 1.00 \\
\hline 61 & $\bar{F}$ & $772-1 \mathrm{~F}$ & 1 & & & 5.00 & $2.82 \mathrm{E}-04$ & 32000 & 6.47E-05 & 2.07E-04 & .73 \\
\hline 62 & $\mathbf{F}$ & FB LINE & 14 & 9 & 54 & 33.00 & $5.98 E-04$ & 30000 & 6.47E-05 & 5.98E-04 & 14.00 \\
\hline 63 & $F$ & NSR/PSF & 0 & & & 3.00 & & 40000 & 6.47E-05 & $2.59 \mathrm{E}-04$ & .55 \\
\hline
\end{tabular}


Table 1

\begin{tabular}{|c|c|c|c|c|c|c|c|c|c|c|c|}
\hline & A & $\mathbf{B}$ & C & D & $E$ & $\mathbf{F}$ & $\mathbf{G}$ & $\mathbf{H}$ & 1 & $J$ & $\mathbf{K}$ \\
\hline 1 & & & & & & & BLDG & & AREA SPECIFIC & ADOPTED VALUE & \# OF FIRES \\
\hline 2 & & & $\$$ FIRES & & & YRS IN & SPECIFIC & & SEVERE & FOR BLDG & EXPECTED \\
\hline 3 & & & REPORTED & MTH & $\mathbf{Y R}$ & SERVICE & SEVERE & BLDG & FIRE FREQ I & SEVERE & BASED ON \\
\hline 4 & AREA & BLDG & $1 / 58-12 / 90$ & COMP & COMP & $1 / 58-12 / 90$ & FIRE FREO & SOFT & $10.000 \mathrm{SQ} \mathrm{FT}$ & FIRE FREQ & BLDG SFREO \\
\hline 5 & & & & & & & & & & & \\
\hline 64 & $\mathbf{G}$ & 647-G & 0 & - & & & & 5000 & 1.84E-05 & $9.20 \mathrm{E}-06$ & \\
\hline 65 & $\mathbf{G}$ & $681-1 \mathrm{G}(1)$ & 1 & 7 & 53 & 33.00 & 4.27E-05 & 12843 & 1.84E-05 & 4.27E-05 & 1.00 \\
\hline 66 & $\mathbf{G}$ & $681-16(2)$ & 1 & 7 & 53 & 33.00 & $4.27 E-05$ & 12843 & 1.84E-05 & 4.27E-05 & 1.00 \\
\hline 67 & $\mathbf{G}$ & $681-3 G(1)$ & 1 & 7 & 53 & 33.00 & 4.27E-05 & 12843 & $1.84 \mathrm{E}-05$ & 4.27E-05 & 1.00 \\
\hline 68 & $\mathbf{G}$ & $681-3 G(2)$ & 1 & 7 & 53 & 33.00 & 4.27E-05 & 12843 & $1.84 \mathrm{E}-05$ & $4.27 E-05$ & 1.00 \\
\hline 69 & $\mathbf{G}$ & $681-5 G$ & 0 & 7 & 53 & 33.00 & & 5899 & 1.84E-05 & $1.09 \mathrm{E}-05$ & .25 \\
\hline 70 & $\mathbf{G}$ & $681-6 G$ & 0 & 12 & 58 & 32.08 & & 2400 & 1.84E-05 & 4.42E-06 & .10 \\
\hline 71 & $\mathbf{G}$ & $709-2 G$ & 0 & 4 & 87 & 3.75 & & 13550 & $1.84 E-05$ & 2.49E-05 & .07 \\
\hline 72 & G & $709-46$ & 0 & 10 & 88 & 2.25 & & 11550 & 1.84E-05 & 2.13E-05 & .03 \\
\hline 73 & $\mathbf{G}$ & $709-G$ & 0 & 7 & 83 & 7.50 & & 10080 & 1.84E-05 & $1.85 \mathrm{E}-05$ & .10 \\
\hline 74 & $\mathbf{G}$ & $714-2 \mathrm{G}$ & 0 & 1 & 56 & 33.00 & & 11956 & $1.84 E-05$ & 2.20E-05 & .52 \\
\hline 75 & G & 714.66 & 0 & 1 & 52 & 33.00 & & 16000 & $1.84 E-05$ & 2.94E-05 & .69 \\
\hline 76 & $\mathrm{H}$ & $211-8 \mathrm{H}$ & 0 & 6 & 87 & 3.58 & & 3000 & 3.97E-05 & 1.19E-05 & .03 \\
\hline 77 & $H$ & $211-9 H \& 10 \mathrm{H}$ & of & 6 & 87 & 3.58 & & 600 & 3.97E-05 & $2.38 E-06$ & .01 \\
\hline 78 & $H$ & $211-H \& 7 H(1)$ & 4 & 7 & 55 & 33.00 & $1.71 \mathrm{E}-04$ & 10000 & 3.97E-05 & 1.71E-04 & 4.00 \\
\hline 79 & $H$ & 211-H87H (2) & 4 & 7 & 55 & 33.00 & 1.71E-04 & 10000 & 3.97E-05 & $1.71 E-04$ & 4.00 \\
\hline 80 & $H$ & $221 \cdot H(1)$ & 23 & 7 & 55 & 33.00 & $9.82 E-04$ & 329600 & 3.97E-05 & 9.82E-04 & 23.00 \\
\hline 81 & $H$ & $221-H(2)$ & 23 & 7 & 55 & 33.00 & $9.82 \mathrm{E}-04$ & 329600 & 3.97E-05 & 9.82E-04 & 23.00 \\
\hline 82 & $\mathrm{H}$ & $230-H$ & 1 & 11 & 83 & 7.17 & $1.97 E-04$ & 7165 & $3.97 \mathrm{E}-05$ & 1.97E-04 & 1.00 \\
\hline 83 & $H$ & $235-4$ & 0 & 9 & 84 & 6.33 & & 12000 & $3.97 \mathrm{E}-05$ & $4.76 \mathrm{E}-05$ & .21 \\
\hline 84 & $\mathrm{H}$ & $241-10 \mathrm{H}$ & 0 & & & & & 9713 & 3.97E-05 & $3.86 E-05$ & \\
\hline 85 & $\mathrm{H}$ & 242-1H & 1 & 3 & 63 & 27.83 & 5.06E-05 & 720 & 3.97E-05 & $5.06 E-05$ & 1.00 \\
\hline 86 & $H$ & 244-H & 1 & 10 & 62 & 28.25 & 4.99E-05 & 20785 & 3.97E-05 & 4.99E-05 & 1.00 \\
\hline 87 & $H$ & 251-H & 0 & 7 & 55 & 33.00 & & 1845 & 3.97E-05 & 7.32E-06 & .17 \\
\hline 88 & $H$ & $253-H$ & 0 & 10 & 86 & 4.25 & & 1350 & $3.97 E-05$ & $5.36 \mathrm{E}-06$ & .02 \\
\hline 89 & $\mathrm{H}$ & 254-28H & 0 & & & & & 30 & 3.97E-05 & $1.19 E-07$ & \\
\hline 90 & $H$ & $284-H(1)$ & 8 & 7 & 55 & 33.00 & $3.41 E-04$ & 2500 & 3.97E-05 & $3.41 E-04$ & 8.00 \\
\hline 91 & $\mathrm{H}$ & $284-H(2)$ & 8 & 7 & 55 & 33.00 & 3.41E-04 & 2500 & 3.97E-05 & 3.41E-04 & 8.00 \\
\hline 92 & $\mathrm{H}$ & $292-H$ & 2 & 7 & 55 & 33.00 & 8.54E-05 & 13251 & $3.97 \mathrm{E}-05$ & $8.54 E-05$ & 2.00 \\
\hline & & & & & & 4.00 & & 11250 & $2.28 \mathrm{E}-05$ & 2.57E-05 & .07 \\
\hline
\end{tabular}




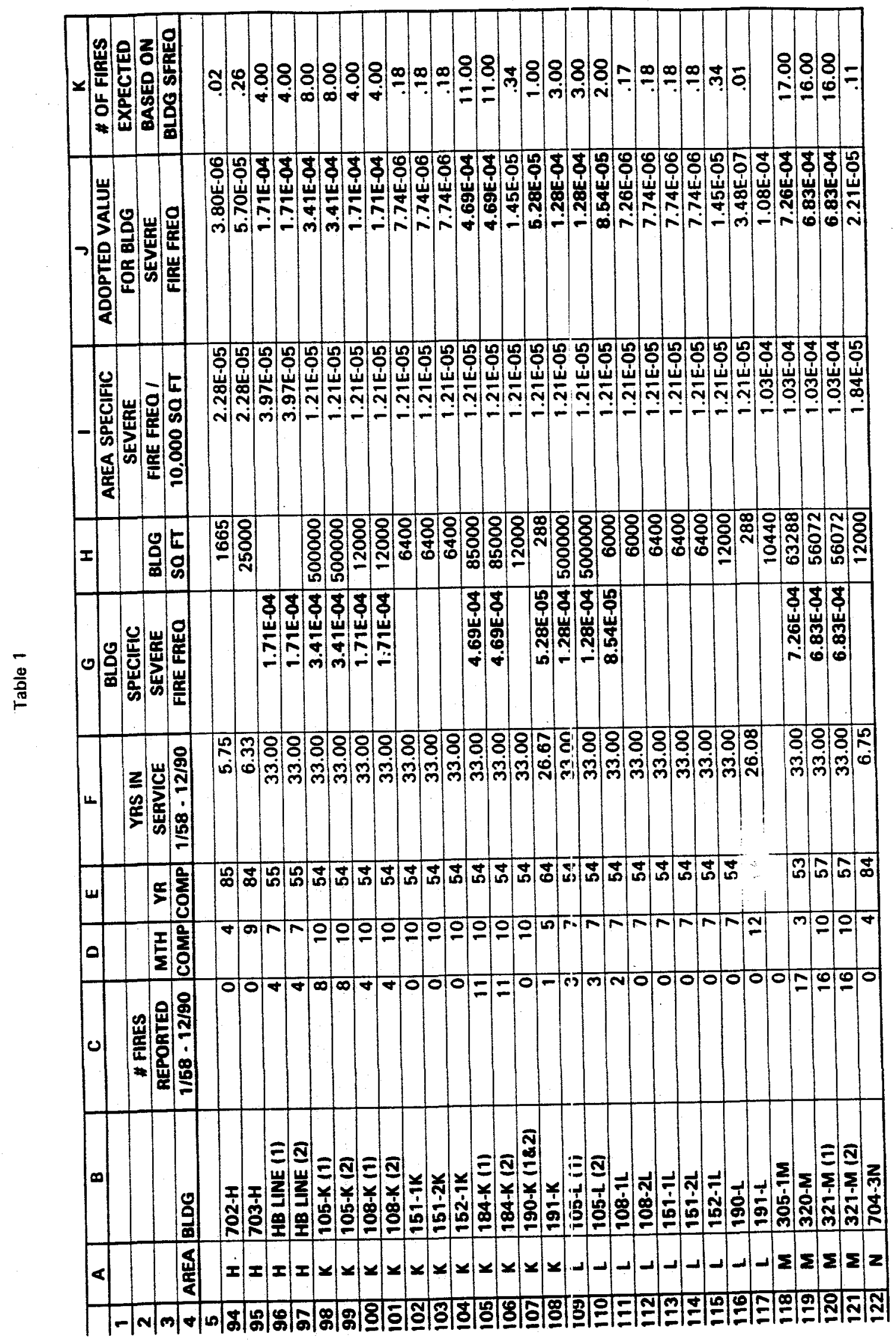


Table 1

\begin{tabular}{|c|c|c|c|c|c|c|c|c|c|c|c|}
\hline & $\mathbf{A}$ & $\mathbf{B}$ & C & D & $\mathbf{E}$ & $\mathbf{F}$ & $\mathbf{G}$ & $H$ & 1 & $\mathrm{~J}$ & $\mathbf{K}$ \\
\hline 1 & & & & & & & BLDG & & AREA SPECIFIC & ADOPTED VALUE & \# OF FIRES \\
\hline 2 & & & * fiaEs & & & YAS IN & SPECIFIC & & SEVERE & FOR BLDG & EXPECTED \\
\hline 3 & & & REPORTED & MTH & YR & SERVICE & SEVERE & BLDG & FIRE FREO I & SEVERE & BASED ON \\
\hline 4 & AREA & BLDG & $1 / 58-12 / 90$ & Comp & CoMP & $1 / 58-12 / 90$ & FIRE FREQ & SOFT & $10,000 \mathrm{SOFT}$ & FIRE FREQ & BLDG SFREO \\
\hline 5 & & & & & & & & & & & \\
\hline 123 & $\mathbf{N}$ & $711-N$ & 1 & 7 & 53 & 33.00 & 4.27E-05 & 45600 & 1.84E-05 & $4.27 E-05$ & 1.00 \\
\hline 124 & $\mathbf{N}$ & $713-1 N$ & 0 & 11 & 82 & 8.17 & & 30000 & 1.84E-05 & $5.52 \mathrm{E}-05$ & .32 \\
\hline 125 & $\mathbf{N}$ & $713-N$ & 1 & 7 & 53 & 33.00 & $4.27 E-05$ & 30000 & $1.84 E-05$ & $4.27 E-05$ & 1.00 \\
\hline 126 & $\mathbf{N}$ & $716-N$ & 0 & 7 & 53 & 33.00 & & 15850 & $1.84 \mathrm{E}-05$ & $2.92 E-05$ & .68 \\
\hline 127 & $\mathbf{N}$ & $717-10 \mathrm{~N}$ & 0 & 7 & 80 & 10.50 & & 12000 & $1.84 \mathrm{E}-05$ & $2.21 E-05$ & .16 \\
\hline 128 & $N$ & $717-11 N$ & 0 & 7 & 53 & 33.00 & & 5265 & $1.84 \mathrm{E}-05$ & $9.69 \mathrm{E}-06$ & .23 \\
\hline 129 & $\mathbf{N}$ & $717-5 N$ & 0 & 7 & 53 & 33.00 & & 22000 & $1.84 E-05$ & $4.05 E-05$ & .95 \\
\hline 130 & $\mathbf{N}$ & $717-8 \mathrm{~N}$ & 0 & 7 & 53 & 33.00 & & 7400 & 1.84E-05 & $1.36 E-05$ & .32 \\
\hline 131 & $N$ & $719-N$ & 0 & 7 & 53 & 33.00 & & 6500 & $1.84 E-05$ & $1.20 \mathrm{E}-05$ & .28 \\
\hline 132 & $N$ & $725-1 N$ & 0 & 5 & 83 & 7.67 & & 4500 & $1.84 \mathrm{E}-05$ & $8.28 E-06$ & .05 \\
\hline 133 & $\mathbf{P}$ & 105-P (1) & 13 & 3 & 54 & 33.00 & 5.55E-04 & 750000 & $1.21 E-05$ & 5.55E-04 & 13.00 \\
\hline 134 & $P$ & $105-P(2)$ & 13 & 3 & 54 & 33.00 & 5.55E-04 & 750000 & $1.21 E-05$ & 5.55E-04 & 13.00 \\
\hline 135 & $P$ & $108-P(1)$ & 3 & 3 & 54 & 33.00 & $1.28 E-04$ & 12000 & $1.21 E-05$ & $1.28 E-04$ & 3.00 \\
\hline 136 & $\mathbf{P}$ & $108-P(2)$ & 3 & 3 & 54 & 33.00 & $1.28 E-04$ & 12000 & 1.21E-05 & $1.28 E-04$ & 3.00 \\
\hline 137 & $\mathbf{P}$ & $151-1 P(1)$ & 0 & 3 & 54 & 33.00 & & 6400 & $1.21 \mathrm{E}-05$ & $7.74 \mathrm{E}-06$ & .18 \\
\hline 138 & $\mathbf{P}$ & $151-1 P(2)$ & 0 & 3 & 54 & 33.00 & & 6400 & 1.21E-05 & $7.74 \mathrm{E}-06$ & .18 \\
\hline 139 & $\mathbf{P}$ & $151-2 P(1)$ & 0 & 3 & 54 & 33.00 & & 6400 & $1.21 E-05$ & $7.74 \mathrm{E}-06$ & .18 \\
\hline 140 & $\mathbf{P}$ & $161-2 P(2)$ & 0 & 3 & 54 & 33.00 & & 6400 & $1.21 E-05$ & $7.74 E-06$ & .18 \\
\hline 141 & $\mathbf{P}$ & $152-1 P$ & 0 & 3 & 54 & 33.00 & & 6400 & $1.21 \mathrm{E}-05$ & $7.74 \mathrm{E}-06$ & .18 \\
\hline 142 & $P$ & $184-P$ & 12 & 3 & 54 & 33.00 & $5.12 E-04$ & 85000 & $1.21 \mathrm{E}-05$ & $5.12 E-04$ & 12.00 \\
\hline 143 & $\mathbf{P}$ & $190-P(1)$ & 0 & 3 & 54 & 33.00 & & 12000 & $1.21 \mathrm{E}-05$ & $1.45 \mathrm{E}-05$ & .34 \\
\hline 144 & P & $190-P(2)$ & 0 & 3 & 54 & 33.00 & & 12000 & $1.21 E-05$ & $1.45 E-05$ & .34 \\
\hline 145 & $\mathbf{P}$ & 191-P & 0 & 3 & 65 & 25.83 & & 288 & $1.21 E-05$ & $3.48 \mathrm{E}-07$ & .01 \\
\hline 146 & $\mathbf{s}$ & $210-5$ & 0 & & & & & 50703 & $5.29 \mathrm{E}-05$ & $2.68 \mathrm{E}-04$ & \\
\hline 147 & $\mathbf{s}$ & $221-5$ & 1 & & & & & 191400 & 5.29E-05 & $1.01 \mathrm{E}-03$ & \\
\hline 148 & $\mathbf{s}$ & $292-5$ & 0 & & & & & 21274 & $5.29 E-05$ & $1.13 \mathrm{E}-04$ & \\
\hline 149 & 5 & $422-5$ & 0 & & & & & 4541 & 5.29E-05 & $2.40 \mathrm{E}-05$ & \\
\hline $15 n$ & 5 & 511.5 & o & & & & & 9593 & $5.29 \mathrm{E}-0.5$ & 5.07E-05 & \\
\hline
\end{tabular}

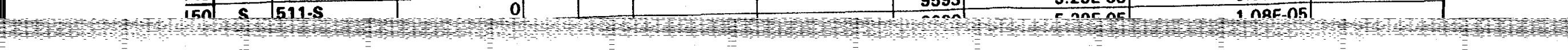


Table 1

\begin{tabular}{|c|c|c|c|c|c|c|c|c|c|c|c|}
\hline & $\mathbf{A}$ & $\mathbf{B}$ & C & D & $\mathbf{E}$ & $\mathbf{F}$ & $\mathbf{G}$ & $\mathrm{H}$ & 1 & $J$ & $\mathbf{K}$ \\
\hline 1 & & & & & & & BLDG & & AREA SPECIFIC & ADOPTED VALUE & * OF FIRES \\
\hline 2 & & & $\because$ FIRES & & & YAS IN & SPECIFIC & & SEVERE & FOR BLDG & EXPECTED \\
\hline 3 & & & AEPORTED & MTH & $\mathbf{Y R}$ & SERVICE & SEVERE & BLDG & FIRE FREQ I & SEVERE & BASED ON \\
\hline 4 & AREA & BLDG & $1 / 58 \cdot 12 / 90$ & COMP & COMP & $1 / 58-12 / 90$ & FIRE FREO & SQFT & $10.000 \mathrm{SQFT}$ & FIRE FAEO & BLDG SFREO \\
\hline 5 & & & & & & & & & & & \\
\hline 152 & $\mathbf{s}$ & $980-5$ & 0 & & & & & 5426 & 5.29E-05 & 2.87E-05 & \\
\hline 153 & $\mathbf{T}$ & 670-T & 0 & 11 & 86 & 4.17 & & 3600 & 1.67E-05 & $6.01 E-06$ & .02 \\
\hline 154 & $T$ & 672-T & 0 & 3 & 84 & 6.83 & & 12120 & 1.67E-05 & 2.02E-05 & .10 \\
\hline 155 & $\mathbf{r}$ & $675-T$ & 1 & 1 & 83 & 8.00 & $1.76 E-04$ & 6642 & 1.67E-05 & 1.76E-04 & 1.00 \\
\hline 156 & $\mathbf{T}$ & 677.T & 0 & 1 & 56 & 33.00 & & 15280 & 1.67 E-05 & 2.55E-05 & .60 \\
\hline 157 & $\mathbf{T}$ & $678-\mathrm{T}$ & 4 & 7 & 53 & 33.00 & 1.71E-04 & 12957 & $1.67 \mathrm{E}-05$ & 1.71E-04 & 4.00 \\
\hline 158 & $\mathbf{T}$ & $704-1 T$ & 0 & 10 & 87 & 3.25 & & 6500 & 1.67E-05 & $1.09 \mathrm{E}-05$ & .03 \\
\hline 169 & $\mathbf{T}$ & 704-T & 0 & 12 & 82 & 8.08 & & 6395 & 1.67E-05 & 1.07E-05 & .06 \\
\hline
\end{tabular}


Table 2

\begin{tabular}{|c|c|c|c|c|}
\hline \multicolumn{3}{|c|}{100 AREAS: AVERAGE SEVERE FIRE } & & \\
\hline \multicolumn{3}{|c|}{ FREQUENCY / 10000 SQ FT } & & \\
\hline BLDG & SOFT & \# FIRES & & \\
\hline $105-\mathrm{C}$ & 500000 & 17 & & \\
\hline $105-6 \mathrm{C}$ & 240 & 0 & & \\
\hline $105-7 \mathrm{C}$ & 240 & 0 & & \\
\hline $108-1 \mathrm{C}$ & 6000 & of & & \\
\hline $108-2 C$ & 6000 & of & & \\
\hline $110-\mathrm{C}$ & 240 & 0 & & \\
\hline $151-1 \mathrm{C}$ & 6400 & 0 & & \\
\hline $151-2 C$ & 6400 & 0 & & \\
\hline $152-7 \mathrm{C}$ & 200 & 0 & & \\
\hline $183-2 \mathrm{C}$ & 760 & 0 & & \\
\hline $183-4 \mathrm{C}$ & 6430 & 0 & & \\
\hline $184-2 C$ & 828 & of & & \\
\hline $184-6 \mathrm{C}$ & 800 & 0 & & \\
\hline $190-\mathrm{C}$ & 12000 & 0 & & \\
\hline $191-C$ & 288 & 0 & & \\
\hline $105-\mathrm{K}$ & 500000 & 8 & & \\
\hline $105-6 \mathrm{~K}$ & 96 & of & & \\
\hline $105-7 \mathrm{~K}$ & 96 & 0 & & \\
\hline $105-8 \mathrm{~K}$ & 120 & 0 & & \\
\hline $105-13 \mathrm{~K}$ & 3250 & 0 & & \\
\hline $108 \cdot 1 \mathrm{~K}$ & 6000 & 3 & & \\
\hline $108-2 \mathrm{~K}$ & 6000 & 1 & & \\
\hline $110-\mathrm{K}$ & 240 & of & & \\
\hline $151-1 \mathrm{~K}$ & 6400 & 0) & & \\
\hline $151-2 \mathrm{~K}$ & 6400 & 0 & & \\
\hline $152-1 \mathrm{~K}$ & 6400 & 0 & & \\
\hline $152-7 \mathrm{~K}$ & 200 & 0 & & \\
\hline $183-2 \mathrm{~K}$ & 720 & 1 & & \\
\hline $183-4 \mathrm{~K}$ & 6430 & 0 & & \\
\hline $184-K$ & 85000 & 11 & & \\
\hline $184-5 K$ & 173 & 0 & & \\
\hline $185-K$ & 375 & 1 & & \\
\hline $190-K$ & 12000 & 0 & & \\
\hline $191-K$ & 288 & 1 & & \\
\hline $105-P$ & 750000 & 13 & & \\
\hline $105-2 \mathrm{P}$ & 720 & 0 & & \\
\hline $105-3 P$ & 720 & 0 & & \\
\hline $105-6 \mathrm{P}$ & 96 & 0 & & \\
\hline $105-13 P$ & 3250 & 0 & & \\
\hline $105.14 \mathrm{P}$ & 360 & 0 & & \\
\hline $108 \cdot 1 P$ & 6000 & 1 & & \\
\hline $108-2 P$ & 6000 & 2 & & \\
\hline $110-\mathrm{P}$ & 240 & o) & & \\
\hline $151-1 \mathrm{P}$ & 6400 & 0 & & \\
\hline $151.2 P$ & 6400 & 의 & & \\
\hline
\end{tabular}


Table 2

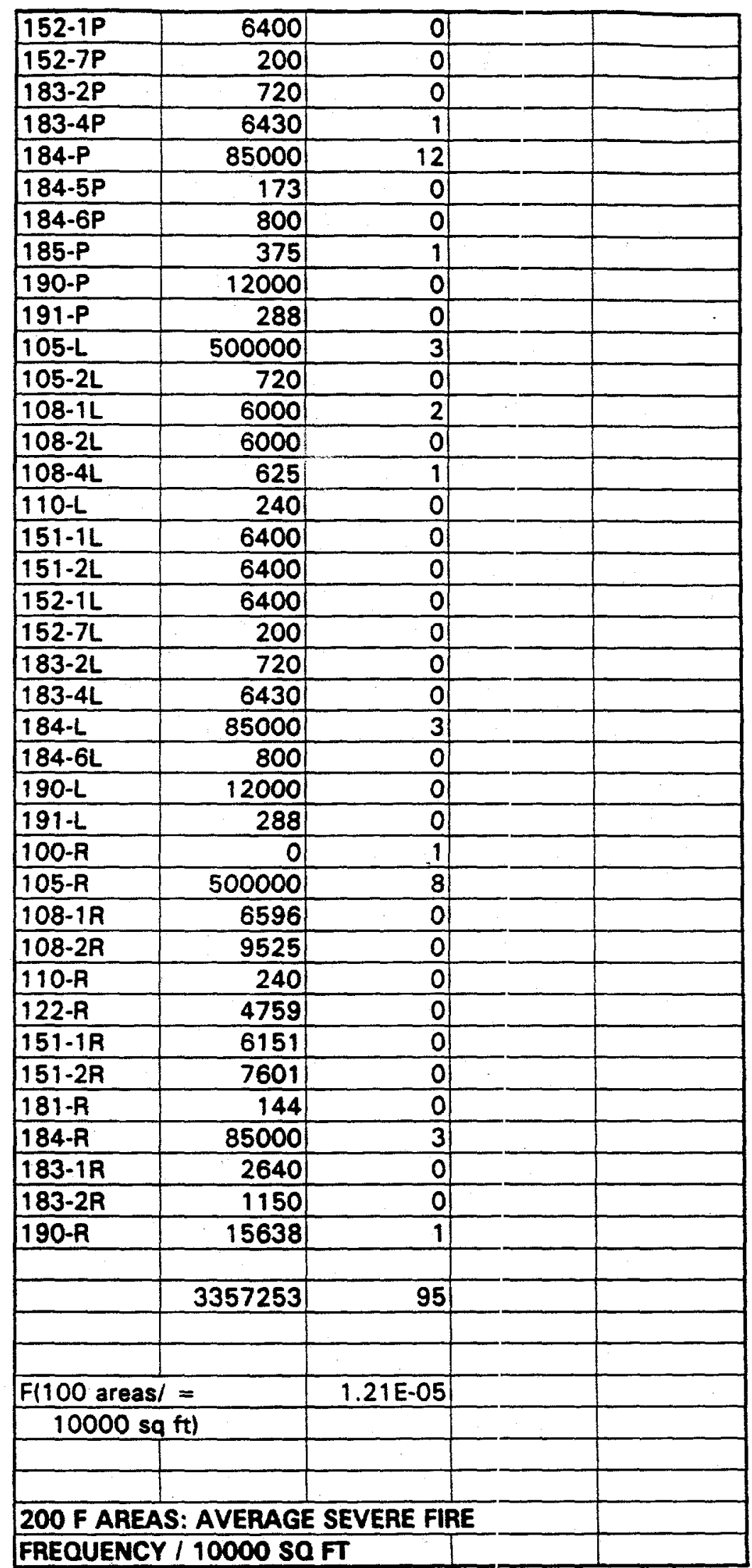


Table 2

\begin{tabular}{|c|c|c|c|c|}
\hline BLDG & SQFT & \# FIRES & & \\
\hline $200-F$ & 0 & 1 & & \\
\hline $211-F$ & 10000 & 3 & & \\
\hline $211-1 F$ & 6000 & 0 & & \\
\hline $211-2 \mathrm{~F}$ & 740 & o) & & \\
\hline $211-3 \mathrm{~F}$ & 1900 & 0 & & \\
\hline $211-4 \mathrm{~F}$ & 140 & 0 & & \\
\hline $211-5 \mathrm{~F}$ & 288 & 0 & & \\
\hline $211-7 F$ & 1800 & 0 & & \\
\hline $217-F$ & 1024 & 0 & & \\
\hline 221-F & 329600 & 88 & & \\
\hline $221.1 \mathrm{~F}$ & 24067 & 1 & & \\
\hline $221-3 F$ & 4000 & 0 & & \\
\hline $221-4 F$ & 192 & 0 & & \\
\hline $221-10 \mathrm{~F}$ & 1536 & 0 & & \\
\hline $221-12 F$ & 10800 & 0 & & \\
\hline $221-13 F$ & 416 & 0 & & \\
\hline $221-15 F$ & 1536 & 0 & & \\
\hline $221-16 \mathrm{~F}$ & 1008 & o & & \\
\hline $221-21 \mathrm{~F}$ & 20000 & 0 & & \\
\hline $221-22 \mathrm{~F}$ & 20000 & 0 & & \\
\hline $221-26 \mathrm{~F}$ & 800 & of & & \\
\hline $221-27 F$ & 1000 & 0 & & \\
\hline $222-F$ & 5989 & 1 & & \\
\hline $223-F$ & 390 & 0 & & \\
\hline $232-F$ & 16440 & 1 & & \\
\hline 235-F & 47080 & 5 & & \\
\hline 235-1F & 950 & o & & \\
\hline $235-2 F$ & 476 & o & & \\
\hline $235-6 \mathrm{~F}$ & 288 & 0 & & \\
\hline 235-9F & 288 & 0 & & \\
\hline $235-11 \mathrm{~F}$ & 1792 & o & & \\
\hline $240-F$ & 3072 & 0 & & \\
\hline $241-F$ & 714 & 1 & & \\
\hline 241-17F & 1078 & 0 & & \\
\hline $241-18 F$ & 1200 & 0 & & \\
\hline $241-24 F$ & 1500 & 1 & & \\
\hline $241-28 F$ & 1000 & of & & \\
\hline $241-51 F$ & 36 & 0 & & \\
\hline $241-53 F$ & 300 & 0 & & \\
\hline $241-58 \mathrm{~F}$ & 2400 & 0 & & \\
\hline $241-62 F$ & 390 & 0 & & \\
\hline $241-64 F$ & 480 & 0 & & \\
\hline $241-65 F$ & 360 & 이 & & \\
\hline $241-72 F$ & 360 & 0 & & \\
\hline 241-80F & 600 & 0 & & \\
\hline $241-84 F$ & 400 & 0 & & \\
\hline $241-91 F$ & 2400 & 0 & & \\
\hline
\end{tabular}


Table 2

\begin{tabular}{|c|c|c|c|c|}
\hline $241-93 F$ & 608 & 0 & & \\
\hline $241-96 \mathrm{~F}$ & 144 & 0 & & \\
\hline $241-100 \mathrm{~F}$ & 1792 & 0 & & \\
\hline $242-1 \mathrm{~F}$ & 913 & o) & & \\
\hline $242-16 \mathrm{~F}$ & 5 & 0 & & \\
\hline $246-F$ & 13660 & of & & \\
\hline $246-1 \mathrm{~F}$ & 240 & 0 & & \\
\hline $246-2 F$ & 1792 & 0 & & \\
\hline $247-F$ & 140000 & 4 & & \\
\hline $247.3 F$ & 1836 & 0 & & \\
\hline $247-4 F$ & 180 & 0 & & \\
\hline $247-7 \mathrm{~F}$ & 600 & of & & \\
\hline $247-8 \mathrm{~F}$ & 276 & 0 & & \\
\hline $247-9 F$ & 240 & 0 & & \\
\hline $247-11 \mathrm{~F}$ & 180 & 0 & & \\
\hline $247-12 \mathrm{~F}$ & 3795 & 0 & & \\
\hline $247-41 F$ & 1200 & 0 & & \\
\hline $251-F$ & 1923 & 1 & & \\
\hline 254-2F & 520 & 0 & & \\
\hline 254-5F & 816 & 0 & & \\
\hline $260-1 F$ & 80 & o) & & \\
\hline $280-1 F$ & 944 & 0 & & \\
\hline $280-2 F$ & 400 & of & & \\
\hline $281-4 F$ & 250 & 0 & & \\
\hline $281-6 \mathrm{~F}$ & 250 & 0 & & \\
\hline $281-12 F$ & 112 & 0 & & \\
\hline $281-19 F$ & 144 & 0 & & \\
\hline $281-20 F$ & 144 & 0 & & \\
\hline $281-21 F$ & 144 & 0 & & \\
\hline $281-22 F$ & 144 & 0 & & \\
\hline $281-23 F$ & 144 & 0 & & \\
\hline $281-24 F$ & 144 & 0 & & \\
\hline $281-25 \mathrm{~F}$ & 144 & 0 & & \\
\hline $282-F$ & 210 & 0 & & \\
\hline 284-F & 30000 & 4 & & \\
\hline $284-2 F$ & 500 & 0 & & \\
\hline 284-3F & 240 & 0 & & \\
\hline $284-4 \mathrm{~F}$ & 840 & 0 & & \\
\hline $284-5 F$ & 240 & 0 & & \\
\hline $284-8 F$ & 2826 & 0 & & \\
\hline $284-9 F$ & 3600 & 0 & & \\
\hline 285-1F & 60 & 0 & & \\
\hline $292-F$ & 12482 & 5 & & \\
\hline $292-1 F$ & 613 & 1 & & \\
\hline $292-2 F$ & 3470 & o) & & \\
\hline \multirow[t]{3}{*}{ 294-1F } & 240 & 0 & & \\
\hline & 755915 & 117 & & \\
\hline & & & & \\
\hline
\end{tabular}


Table 2

\begin{tabular}{|c|c|c|c|c|}
\hline \multicolumn{2}{|c|}{$F(200 \mathrm{~F}$ area $)=$} & 6.61E-05 & & \\
\hline \multicolumn{5}{|c|}{$10000 \mathrm{sq} f \mathrm{ft}$} \\
\hline & & & & \\
\hline & & & & \\
\hline \multicolumn{5}{|c|}{200 H AREAS: AVERAGE SEVERE FIRE } \\
\hline \multirow{2}{*}{\multicolumn{5}{|c|}{\begin{tabular}{|l|l|} 
FREQUENCY / 10000 SQ FT & \\
\end{tabular}}} \\
\hline & & & & \\
\hline BLDG & SQ FT & \# FIRES & & \\
\hline $200-\mathrm{H}$ & a) & 1 & & \\
\hline $211 \cdot 2 \mathrm{H}$ & 1500 & 0 & & \\
\hline $211-4 \mathrm{H}$ & 141 & 0 & & \\
\hline $211-7 \mathrm{H}$ & 10000 & 4 & & \\
\hline $211-8 \mathrm{H}$ & 3000 & 0 & & \\
\hline $211-9 \mathrm{H}$ & 300 & 0 & & \\
\hline $211-10 \mathrm{H}$ & 300 & 0 & & \\
\hline $217 \cdot \mathrm{H}$ & 901 & of & & \\
\hline $221-H$ & 329600 & 27 & & \\
\hline $221-4 \mathrm{H}$ & 1500 & of & & \\
\hline $221-6 \mathrm{H}$ & 240 & 0 & & \\
\hline $221-12 \mathrm{H}$ & 160 & 0 & & \\
\hline $221-13 \mathrm{H}$ & 600 & 0 & & \\
\hline $221-14 \mathrm{H}$ & 600 & of & & \\
\hline $225.1 \mathrm{H}$ & 896 & 0 & & \\
\hline $225-2 \mathrm{H}$ & 1792 & 0 & & \\
\hline $225-3 \mathrm{H}$ & 1792 & 0 & & \\
\hline $225-7 \mathrm{H}$ & 1792 & 0 & & \\
\hline $225-8 \mathrm{H}$ & 1792 & 0 & & \\
\hline $225-9 \mathrm{H}$ & 840 & 0 & & \\
\hline $225-10 \mathrm{H}$ & 960 & 0 & & \\
\hline $225.11 \mathrm{H}$ & 960 & 0 & & \\
\hline $225.12 \mathrm{H}$ & 960 & o & & \\
\hline $225-13 \mathrm{H}$ & 960 & 0 & & \\
\hline $225-14 \mathrm{H}$ & 960 & 0 & & \\
\hline $225-15 \mathrm{H}$ & 960 & 0) & & \\
\hline $225-17 \mathrm{H}$ & 960 & 0 & & \\
\hline $225-18 \mathrm{H}$ & 504 & 0 & & \\
\hline $230-\mathrm{H}$ & 7165 & 1 & & \\
\hline $232-H$ & 55220 & 9 & & \\
\hline $232-1 \mathrm{H}$ & 11000 & 0 & & \\
\hline $233.4 \mathrm{H}$ & 1792 & 0 & & \\
\hline $233-6 \mathrm{H}$ & 1792 & 0 & & \\
\hline $233-7 \mathrm{H}$ & 1792 & 0 & & \\
\hline $233.8 \mathrm{H}$ & 1792 & 0 & & \\
\hline $233-9 H$ & 1792 & 0 & & \\
\hline $233-10 \mathrm{H}$ & 1792 & 0 & & \\
\hline $233-11 \mathrm{H}$ & 432 & 0 & & \\
\hline $233.12 \mathrm{H}$ & 288 & 0 & & \\
\hline
\end{tabular}


Table 2

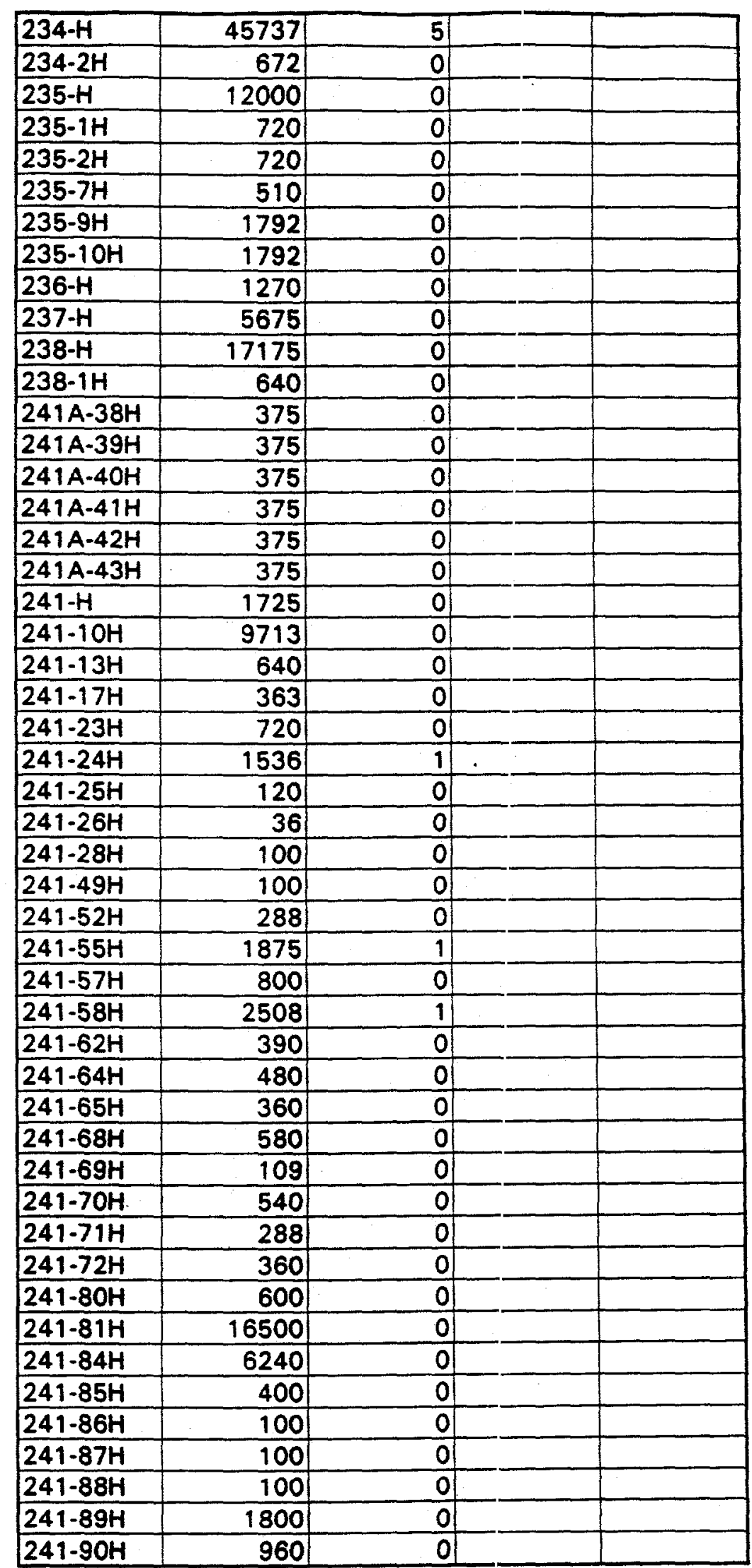


Table 2

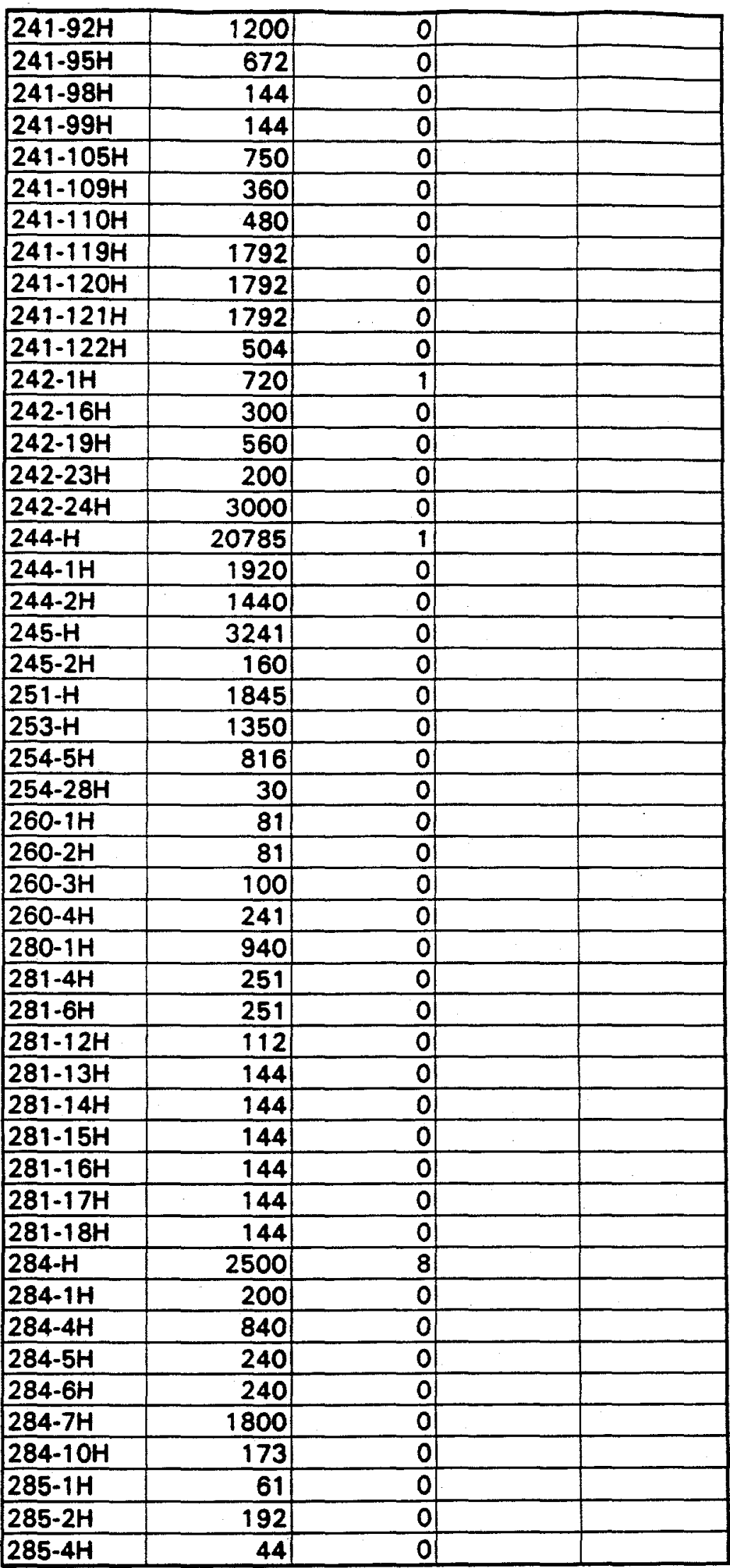


Table 2

\begin{tabular}{|c|c|c|c|c|}
\hline $292-H$ & 13251 & 2 & & \\
\hline $292-2 \mathrm{H}$ & 3360 & 0 & & \\
\hline $292-3 \mathrm{H}$ & 100 & 0) & & \\
\hline $299-H$ & 1200 & 0 & & \\
\hline $299-2 \mathrm{H}$ & 576 & 0 & & \\
\hline 299-3H & 200 & of & & \\
\hline $299-4 \mathrm{H}$ & 1500 & 0 & & \\
\hline \multirow[t]{3}{*}{$299-5 \mathrm{H}$} & 608 & 0 & & \\
\hline & & & & \\
\hline & 667214 & 62 & & \\
\hline \multicolumn{2}{|c|}{$\mathrm{F}(200 \mathrm{H}$ area $)=$} & $3.97 E-05$ & & \\
\hline \multicolumn{2}{|c|}{$10000 \mathrm{sq} f t)$} & & & \\
\hline & & & & \\
\hline & & & & \\
\hline \multirow{2}{*}{\multicolumn{3}{|c|}{\begin{tabular}{|l} 
A AREA: AVERAGE SEVERE FIRE \\
FREQUENCY $/ 10000$ SO FT
\end{tabular}}} & & \\
\hline & & & & \\
\hline \multicolumn{2}{|c|}{ FREQUENCY / 10000 SQ FT } & & & \\
\hline BLDG & SQFT & \# FIRES & & \\
\hline $305-A$ & 18606 & of & & \\
\hline $607-2 A$ & 240 & 2 & & \\
\hline $607-16 \mathrm{~A}$ & 600 & 0 & & \\
\hline $614-1 A$ & 86 & 0 & & \\
\hline $701-1 \mathrm{~A}$ & 873 & 0 & & \\
\hline $701-2 A$ & 170 & 0 & & \\
\hline $701-3 A$ & 150 & 0 & & \\
\hline $701-9 \mathrm{~A}$ & 64 & of & & \\
\hline $701-12 A$ & 1216 & 0 & & \\
\hline $701-13 A$ & 48 & 0 & & \\
\hline $702-A$ & 5195 & 2 & & \\
\hline 703-A & 177000 & 7 & & \\
\hline $703-2 \mathrm{~A}$ & 1536 & 0 & & \\
\hline $703-3 A$ & 1536 & of & & \\
\hline $703-4 A$ & 1560 & 0 & & \\
\hline $703-5 A$ & 1560 & 0 & & \\
\hline $703-6 \mathrm{~A}$ & 1536 & 0 & & \\
\hline $703-9 A$ & 823 & 0 & & \\
\hline $703-10 \mathrm{~A}$ & 1536 & 0. & & \\
\hline $703-11 \mathrm{~A}$ & 1536 & 0 & & \\
\hline $703-12 A$ & 720 & 0 & & \\
\hline $703-13 A$ & 720 & 0 & & \\
\hline $703-14 A$ & 1536 & 이 & & \\
\hline $703-15 A$ & 720 & 0 & & \\
\hline $703-16 \mathrm{~A}$ & 720 & 0 & & \\
\hline $703-17 \mathrm{~A}$ & 720 & O) & & \\
\hline $703-18 A$ & 720 & 0 & & \\
\hline $703-22 A$ & 1792 & 0 & & \\
\hline $703-26 \mathrm{~A}$ & 1792 & of & I & \\
\hline
\end{tabular}


Table 2

\begin{tabular}{|c|c|c|}
\hline $703-28 A$ & 1792 & 0 \\
\hline 703-30A & 1792 & 0 \\
\hline 703-31A & 1792 & 0 \\
\hline $703-33 A$ & 1536 & 0 \\
\hline $703-34 A$ & 1600 & 0 \\
\hline 703-37A & 1600 & 0 \\
\hline 703-38A & 1600 & 0 \\
\hline $703-41 \mathrm{~A}$ & 20000 & 0 \\
\hline $703-42 A$ & 20000 & 0 \\
\hline $703-43 A$ & 20000 & 4 \\
\hline $703-44 A$ & 20000 & 1 \\
\hline $703-45 \mathrm{~A}$ & 20000 & 0 \\
\hline $703-51 \mathrm{~A}$ & 432 & 0 \\
\hline $703.52 \mathrm{~A}$ & 896 & 0 \\
\hline $703-53 \mathrm{~A}$ & 896 & 0 \\
\hline 703-54A & 896 & 0 \\
\hline $703-55 A$ & 896 & 0 \\
\hline 703-56A & 896 & 0 \\
\hline $703.57 \mathrm{~A}$ & 896 & 0 \\
\hline $703-58 A$ & 896 & 0 \\
\hline $703-63 A$ & 896 & 0 \\
\hline 703-70A & 896 & 0 \\
\hline 703-71A & 600 & 0 \\
\hline $703-73 A$ & 1792 & 0 \\
\hline 706-A & 1216 & 0 \\
\hline 707-A & 1008 & 0 \\
\hline 708-A & 40068 & 7 \\
\hline 709-A & 3695 & 0 \\
\hline $710-A$ & 9533 & 2 \\
\hline $711-A$ & 2512 & 0 \\
\hline $712-A$ & 683 & 0 \\
\hline 713-A & 48521 & 0 \\
\hline 713-1A & 11760 & 1 \\
\hline $713-2 A$ & 20000 & 0 \\
\hline $713-3 A$ & 896 & 0 \\
\hline 714-A & 45036 & 0 \\
\hline $715-A$ & 229 & 1 \\
\hline 716-A & 100 & 7 \\
\hline $716-1 A$ & 896 & 0 \\
\hline $716-2 A$ & 7225 & 2 \\
\hline $716-3 A$ & 360 & 0 \\
\hline $716.4 \mathrm{~A}$ & 2400 & 0 \\
\hline $717-A$ & 52435 & 9 \\
\hline $717-4 \mathrm{~A}$ & 200 & 0 \\
\hline $717-5 \mathrm{~A}$ & 1536 & 0 \\
\hline $717-6 \mathrm{~A}$ & 160 & 0 \\
\hline 717-7A & 1536 & 0 \\
\hline $717.8 A$ & 792 & 0 \\
\hline $717.9 \mathrm{~A}$ & 792 & o) \\
\hline
\end{tabular}


Tabie 2

\begin{tabular}{|c|c|c|c|c|}
\hline $717-10 \mathrm{~A}$ & 1440 & 0 & & \\
\hline $719-A$ & 26487 & 0 & & \\
\hline $719-1 \mathrm{~A}$ & 840 & 0 & & \\
\hline $719-2 A$ & 1792 & 0 & & \\
\hline $719-3 A$ & 160 & 0 & & \\
\hline $719-4 A$ & 16000 & 0 & & \\
\hline 719-7A & 896 & 0 & & \\
\hline $719-8 A$ & 896 & 1 & & \\
\hline $719-9 \mathrm{~A}$ & 896 & 0 & & \\
\hline $719-13 A$ & 504 & 0 & & \\
\hline $719-14 A$ & 952 & 0 & & \\
\hline $719-15 A$ & 952 & 0 & & \\
\hline $719-16 \mathrm{~A}$ & 952 & 0 & & \\
\hline $719-17 \mathrm{~A}$ & 952 & 0 & & \\
\hline $719-18 \mathrm{~A}$ & 1792 & 0 & & \\
\hline $719-19 A$ & 1792 & 0 & & \\
\hline $719-20 A$ & 720 & 0 & & \\
\hline $720-A$ & 11180 & 0 & & \\
\hline $721-A$ & 4595 & of & & \\
\hline $722-A$ & 11700 & 0 & & \\
\hline $722-1 \mathrm{~A}$ & 6900 & 0 & & \\
\hline $722-2 A$ & 720 & 0 & & \\
\hline $722.4 \mathrm{~A}$ & 7800 & 0 & & \\
\hline $722-5 \mathrm{~A}$ & 20000 & 0 & & \\
\hline $722.8 \mathrm{~A}$ & 792 & 0 & & \\
\hline $723-A$ & 25194 & 3 & & \\
\hline $723-2 A$ & 1152 & 0 & & \\
\hline $723-4 A$ & 1792 & 0 & & \\
\hline $723-5 \mathrm{~A}$ & 384 & 0 & & \\
\hline $723-6 \mathrm{~A}$ & 1320 & 0 & & \\
\hline $723.7 \mathrm{~A}$ & 1560 & 0 & & \\
\hline $723-8 \mathrm{~A}$ & 720 & 0 & & \\
\hline $723-9 A$ & 720 & 0 & & \\
\hline $723-12 A$ & 240 & 0 & & \\
\hline $723-13 A$ & 210 & 0 & & \\
\hline $723-15 A$ & 2400 & 0 & & \\
\hline $724-A$ & 11595 & 0 & & \\
\hline $724-2 A$ & 2160 & 0 & & \\
\hline $724-3 A$ & 1536 & 0 & & \\
\hline $724-4 A$ & 1536 & 0 & & \\
\hline $724-6 A$ & 384 & 0 & & \\
\hline $724-7 A$ & 384 & 0 & & \\
\hline $725-A$ & 1680 & 0 & & \\
\hline $726-A$ & 400 & 0 & & \\
\hline $730-A$ & 20000 & 1 & & \\
\hline $730-1 \mathrm{~A}$ & 1792 & 0 & & \\
\hline $730-2 A$ & 1792 & 0 & & \\
\hline $730-3 A$ & 1792 & 0 & & \\
\hline $733-A$ & 1926 & 0 & & \\
\hline
\end{tabular}


Table 2

\begin{tabular}{|c|c|c|c|c|}
\hline $733-1 \mathrm{~A}$ & 4000 & 0 & & \\
\hline 734-A & 2034 & 0] & & \\
\hline $735-A$ & 48290 & 13 & & \\
\hline $735-2 A$ & 1800 & 0 & & \\
\hline $735-4 A$ & 213 & 0 & & \\
\hline 735-7A & 7500 & 0 & & \\
\hline $735-8 A$ & 1536 & 0 & & \\
\hline $735-9 A$ & 1536 & 0 & & \\
\hline $735-11 \mathrm{~A}$ & 30000 & 0) & & \\
\hline $735-13 A$ & 1800 & 이 & & \\
\hline $735-16 A$ & 1792 & 0 & & \\
\hline $736-A$ & 10286 & 이 & & \\
\hline $736-1 A$ & 120 & 0 & & \\
\hline $737-A$ & 27321 & 0 & & \\
\hline $737-1 \mathrm{~A}$ & 2702 & o & & \\
\hline $737-2 A$ & 1210 & 0 & & \\
\hline $737-3 A$ & 1356 & 0 & & \\
\hline $737-4 A$ & 740 & 0 & & \\
\hline $737-5 A$ & 2400 & 0 & & \\
\hline $737-6 \mathrm{~A}$ & 1584 & 0 & & \\
\hline $737.7 \mathrm{~A}$ & 1440 & 0 & & \\
\hline $737-8 \mathrm{~A}$ & 1440 & o & & \\
\hline $737-11 \mathrm{~A}$ & 1475 & 0 & & \\
\hline $737-12 A$ & 738 & 0 & & \\
\hline $737-13 \mathrm{~A}$ & 5000 & 0 & & \\
\hline $737-14 A$ & 1440 & 0 & & \\
\hline $737-15 A$ & 1440 & o & & \\
\hline $737.17 \mathrm{~A}$ & 500 & o & & \\
\hline $737-18 A$ & 2400 & 0 & & \\
\hline $737-19 A$ & 1440 & 0 & & \\
\hline $737-20 A$ & 768 & 0 & & \\
\hline $737-23 A$ & 300 & 0 & & \\
\hline $738-A$ & 96 & 0 & & \\
\hline $740-A$ & 3026 & 0 & & \\
\hline $740-3 A$ & 1792 & O & & \\
\hline $740-4 A$ & 1792 & 0 & & \\
\hline $740-5 A$ & 1792 & 0 & & \\
\hline $740-6 \mathrm{~A}$ & 1792 & 0 & & \\
\hline $740-7 A$ & 504 & 0 & & \\
\hline $740-8 A$ & 1200 & 0 & & \\
\hline $740-9 A$ & 1792 & 0 & & \\
\hline $740-10 \mathrm{~A}$ & 1792 & 0 & & \\
\hline $740-11 \mathrm{~A}$ & 1792 & 0 & & \\
\hline $740-12 A$ & 1792 & 0 & & \\
\hline $742-A$ & 29000 & 0 & & \\
\hline $742-2 A$ & 1792 & 0 & & \\
\hline $742-3 A$ & 1792 & 0 & & \\
\hline $742-4 A$ & 720 & 0 & & \\
\hline $742-5 A$ & 720 & 0 & & \\
\hline
\end{tabular}


Table 2

\begin{tabular}{|c|c|c|c|c|}
\hline 743-A & 1034 & 0 & & \\
\hline $743-1 \mathrm{~A}$ & 1440 & 0 & & \\
\hline 745-A & 11025 & 1 & & \\
\hline $745-3 A$ & 384 & 0 & & \\
\hline $745-6 A$ & 2400 & 0 & & \\
\hline $746-A$ & 1296 & 0 & & \\
\hline $746-1 \mathrm{~A}$ & 1200 & 0 & & \\
\hline 748-A & 5680 & 0 & & \\
\hline $748-1 \mathrm{~A}$ & 35 & 0 & & \\
\hline $749-A$ & 11500 & 0 & & \\
\hline $749-1 \mathrm{~A}$ & 100 & 0 & & \\
\hline $751.1 \mathrm{~A}$ & 4450 & 1 & & \\
\hline $751-2 A$ & 288 & 0 & & \\
\hline $752-25 A$ & 200 & 0 & & \\
\hline $761-A$ & 141 & 0 & & \\
\hline $763-A$ & 2400 & 0 & & \\
\hline $770-A$ & 1404 & 0 & & \\
\hline $771-\mathrm{A}$ & 45 & 0 & & \\
\hline 773-A & 304000 & 96 & & \\
\hline $773-2 \mathrm{~A}$ & 1012 & 0 & & \\
\hline $773-3 A$ & 449 & 0 & & \\
\hline $773-4 A$ & 35 & 0 & & \\
\hline $773-5 A$ & 35 & 0 & & \\
\hline $773-6 A$ & 35 & 0 & & \\
\hline $773-8 \mathrm{~A}$ & 35 & 0 & & \\
\hline $773-11 \mathrm{~A}$ & 1824 & 1 & & \\
\hline $773-12 \mathrm{~A}$ & 1632 & 0 & & \\
\hline $773-13 A$ & 1500 & 0 & & \\
\hline $773-14 A$ & 768 & 0 & & \\
\hline $773-16 \mathrm{~A}$ & 1536 & 0 & & \\
\hline $773-17 \mathrm{~A}$ & 1536 & 0 & & \\
\hline $773-18 A$ & 1536 & 0) & & \\
\hline $773-19 A$ & 1536 & 0 & & \\
\hline $773-20 \mathrm{~A}$ & 896 & 0 & & \\
\hline $773.21 \mathrm{~A}$ & 1512 & 0 & & \\
\hline $773-22 A$ & 1536 & 0 & & \\
\hline $773-23 A$ & 1536 & 0 & & \\
\hline $773-24 A$ & 1536 & of & & \\
\hline $773-26 A$ & 1536 & 0 & & \\
\hline $773-27 A$ & 1536 & 0 & & \\
\hline $773-41 \mathrm{~A}$ & 20000 & 0) & & \\
\hline $773-42 A$ & 20000 & 0 & & \\
\hline $773-43 A$ & 18720 & 0 & & \\
\hline 773-51A & 20000 & 0 & & \\
\hline $773-53 A$ & 1792 & 0 & & \\
\hline $773-54 A$ & 1792 & 0 & & \\
\hline $773-55 \mathrm{~A}$ & 1792 & 0 & & \\
\hline 773-56A & 1792 & 0 & & \\
\hline $773-57 \mathrm{~A}$ & 1792 & 0 & & \\
\hline
\end{tabular}


Table 2

\begin{tabular}{|c|c|c|c|}
\hline $773-58 A$ & 1792 & 0 & \\
\hline $773-59 A$ & 1792 & 0 & \\
\hline 773-60A & 504 & 0 & \\
\hline 774-A & 2501 & 1 & \\
\hline $774-1 \mathrm{~A}$ & 720 & 0 & \\
\hline $774-2 \mathrm{~A}$ & 798 & 0 & \\
\hline 775-A & 819 & 0 & \\
\hline $776-A$ & 3000 & 0 & \\
\hline $776-1 \mathrm{~A}$ & 1801 & 0 & \\
\hline $776-3 A$ & 280 & 0 & \\
\hline $776-4 A$ & 280 & 0 & \\
\hline $776.5 \mathrm{~A}$ & 360 & 0 & \\
\hline $776-6 A$ & 2563 & 0 & \\
\hline $776-8 A$ & 608 & 0 & \\
\hline 777-A & 2800 & 0 & \\
\hline $777-10 A$ & 55308 & 1 & \\
\hline $777-11 \mathrm{~A}$ & 96 & 0 & \\
\hline $777-12 \mathrm{~A}$ & 885 & 0 & \\
\hline $777-15 A$ & 720 & o & \\
\hline 778-A & 144 & 이 & \\
\hline 779-A & 4589 & 1 & \\
\hline $779-1 \mathrm{~A}$ & 720 & 요 & \\
\hline $779-3 A$ & 150 & 0 & \\
\hline $779-6 A$ & 288 & 0 & \\
\hline $779-10 \mathrm{~A}$ & 1450 & 0 & \\
\hline $780-1 \mathrm{~A}$ & 270 & 0 & \\
\hline $780-2 A$ & 421 & o & \\
\hline 784-A & 6000 & 2 & \\
\hline $784-4$ & 480 & 0 & \\
\hline $784-1 \mathrm{~A}$ & 960 & of & \\
\hline $784-3 A$ & 150 & 0 & \\
\hline $784-4 A$ & 173 & 0 & \\
\hline 785-A & 960 & 0 & \\
\hline $785-1 \mathrm{~A}$ & 121 & 0 & \\
\hline 786-A & 4600 & 0 & (Bldg 773-A removed \\
\hline $786-1 A$ & 1536 & 0 & from the data because \\
\hline $786-2 A$ & 408 & 0 & it is an outfior) \\
\hline $786-4 A$ & 288 & 0 & \\
\hline 786-5A & 2100 & 0 & \\
\hline $786-6 A$ & 1120 & of & \\
\hline $786-13 A$ & 144 & 0 & \\
\hline 789-A & 6000 & 2 & \\
\hline 792-A & 882 & 0 & \\
\hline \multirow[t]{3}{*}{$905-98 \mathrm{~A}$} & 196 & 0 & \\
\hline & & & \\
\hline & 1597702 & 169 & \\
\hline & & & \\
\hline FlA area) $=$ & & $2.41 E-05$ & \\
\hline $10000 \mathrm{so}$ & & & \\
\hline
\end{tabular}


Table 2

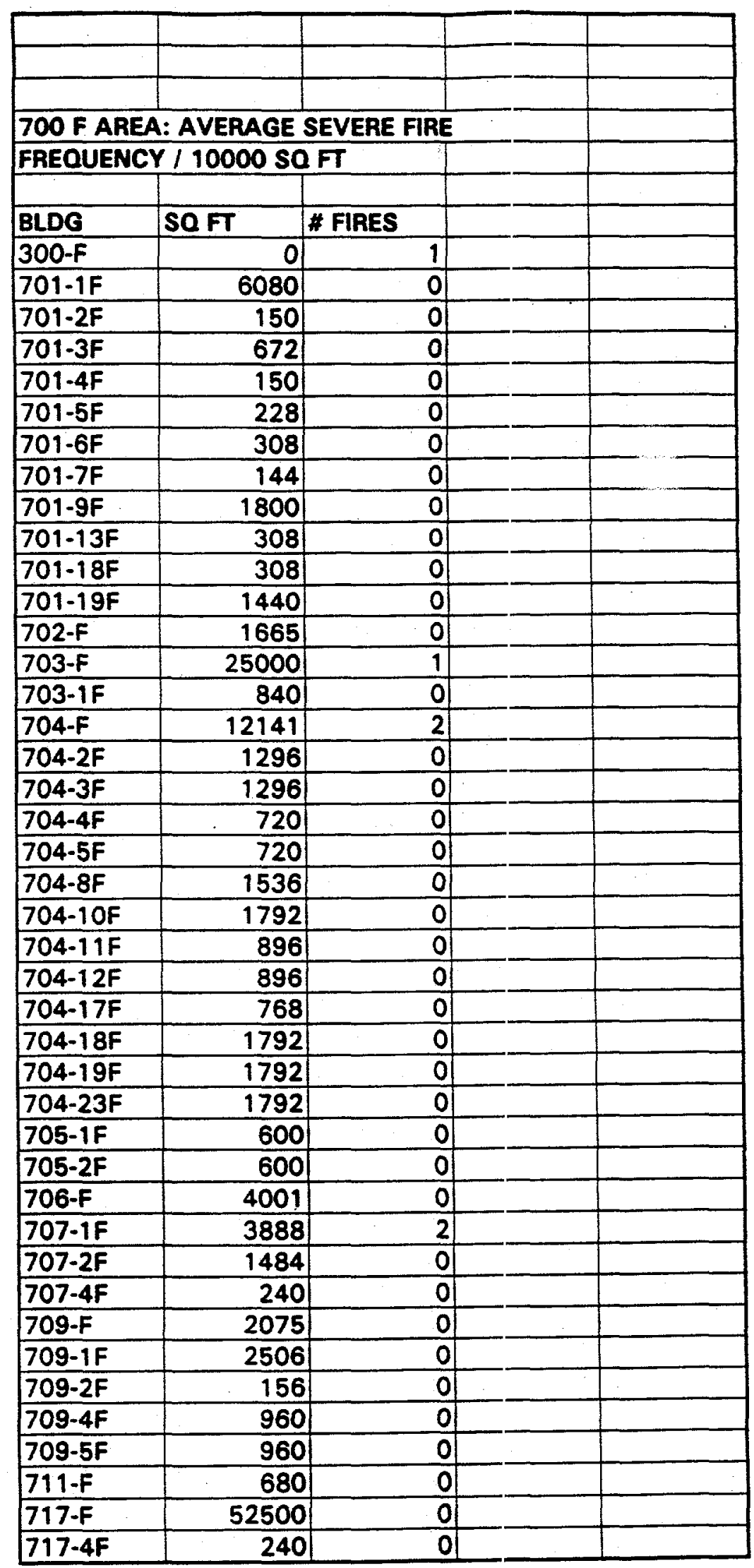


Table 2

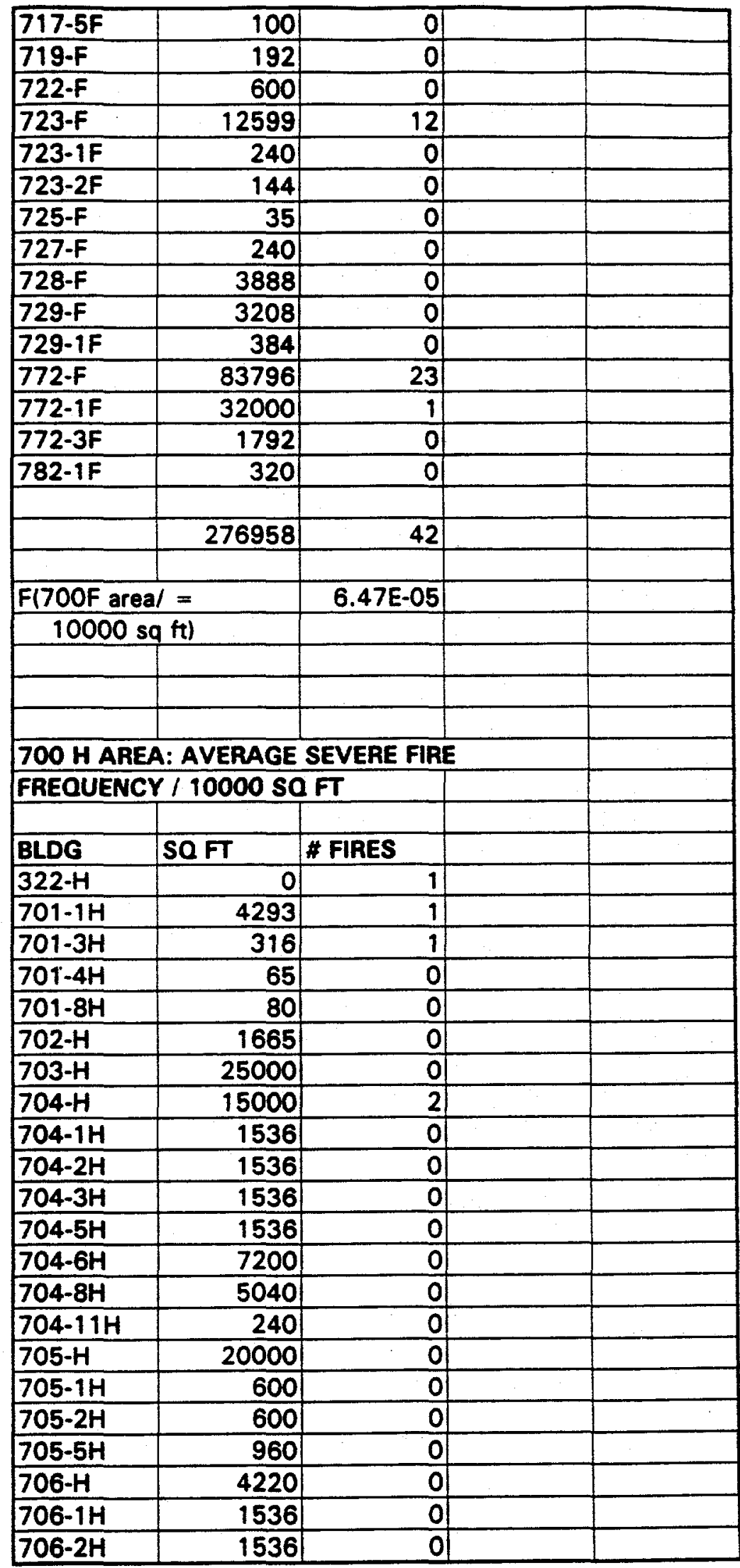


Table 2

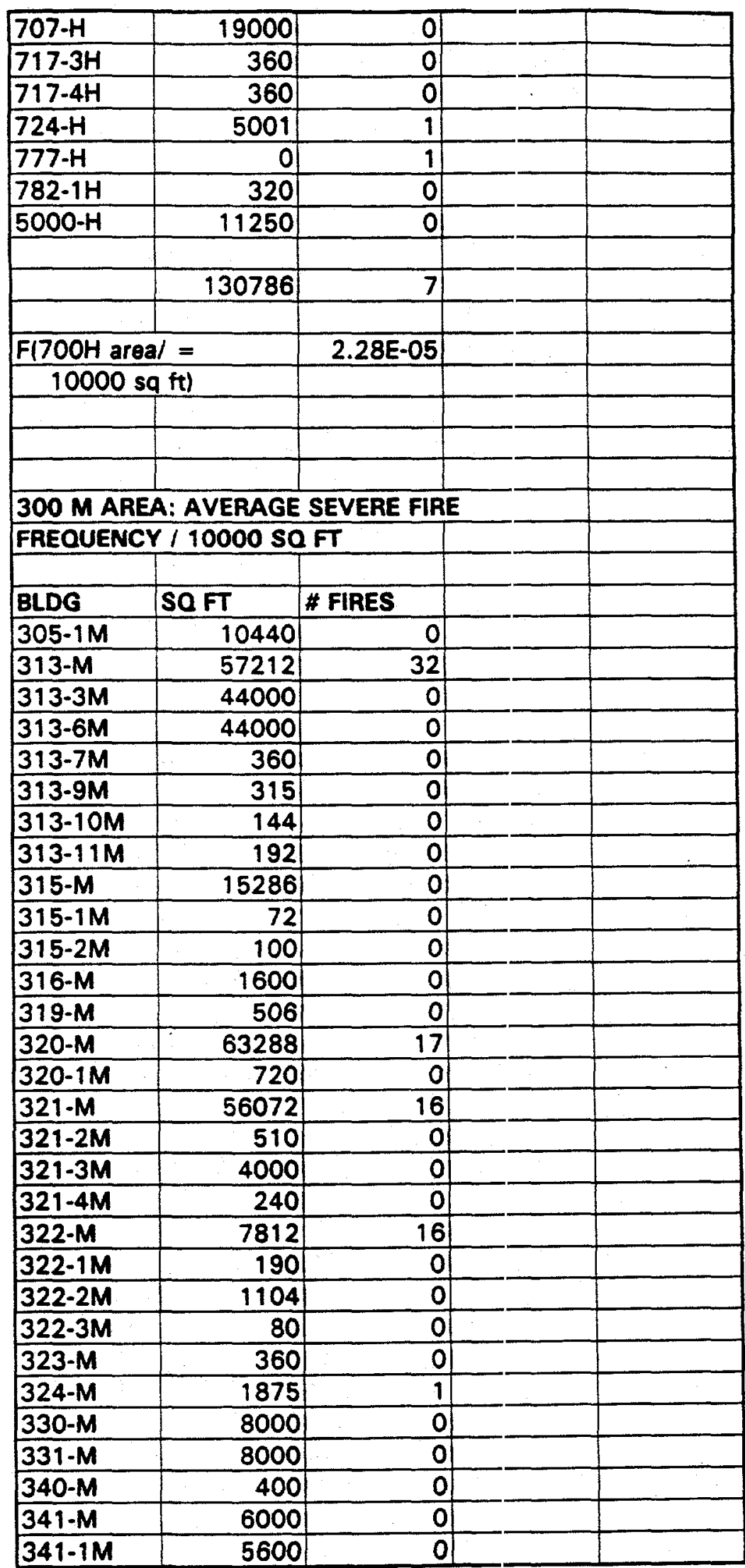


Table 2

\begin{tabular}{|c|c|c|c|c|}
\hline $341-5 M$ & 400 & 0 & & \\
\hline & 338878 & 82 & & \\
\hline & & & & \\
\hline $\mathrm{F}(300 \mathrm{M}$ a & $a /=$ & $1.03 E-04$ & & \\
\hline 10000 & $\mathrm{~g}(\mathrm{ft})$ & & & \\
\hline & & & & \\
\hline & & & & \\
\hline 400 D AR & A: AVERAGE & SEVERE FIRE & & \\
\hline FREQUEN & $Y / 10000 \mathrm{SO}$ & $2 \mathrm{FT}$ & & \\
\hline & & & & \\
\hline BLDG & SOFT & \# FIRES & & \\
\hline $400-D$ & o & 2 & & \\
\hline $401-10$ & 1930 & 2 & & \\
\hline $401-2 D$ & 300 & 0 & & \\
\hline 402-D & 7500 & 1 & & \\
\hline $411-3 D$ & 7464 & 2 & & \\
\hline $411-4 D$ & 352 & of & & \\
\hline $411.6 D$ & 360 & 0 & & \\
\hline $411-70$ & 240 & 0 & & \\
\hline $412-10$ & 1000 & 33 & & \\
\hline 412.20 & 2402 & 0 & & \\
\hline $412-3 D$ & 5790 & 0 & & \\
\hline $412.4 D$ & 1098 & 0 & & \\
\hline $412.5 D$ & 1200 & 0 & & \\
\hline $412-60$ & 1 & 0 & & \\
\hline $412-8 D$ & 631 & 0 & & \\
\hline $412-9 D$ & 366 & 이 & & \\
\hline $412-110$ & 100 & 0 & & \\
\hline $413-D$ & 0 & 8 & & \\
\hline 414-D & 1200 & of & & \\
\hline $415-D$ & 1200 & 0 & & \\
\hline $420-D$ & 6030 & 0 & & \\
\hline $420-2 D$ & 880 & 0 & & \\
\hline $421-D$ & 8128 & 2 & & \\
\hline $421-2 D$ & 5525 & 0 & & \\
\hline $421-4 D$ & 3600 & 0 & & \\
\hline $451-D$ & 566 & 0 & & \\
\hline $480-2 D$ & 270 & 0 & & \\
\hline $480-3 D$ & 576 & 0 & & \\
\hline 483-1D & 38000 & 0 & & \\
\hline $483-2 D$ & 851 & 0 & & \\
\hline $483-3 D$ & 1920 & of & & \\
\hline $484-D$ & 250000 & 24 & & \\
\hline $484-2 D$ & 648 & 1 & & \\
\hline $484-3 D$ & 550 & 0 & & \\
\hline $484-4 D$ & 9200 & 0 & & \\
\hline 484 & 240 & 0 & & \\
\hline
\end{tabular}


Table 2

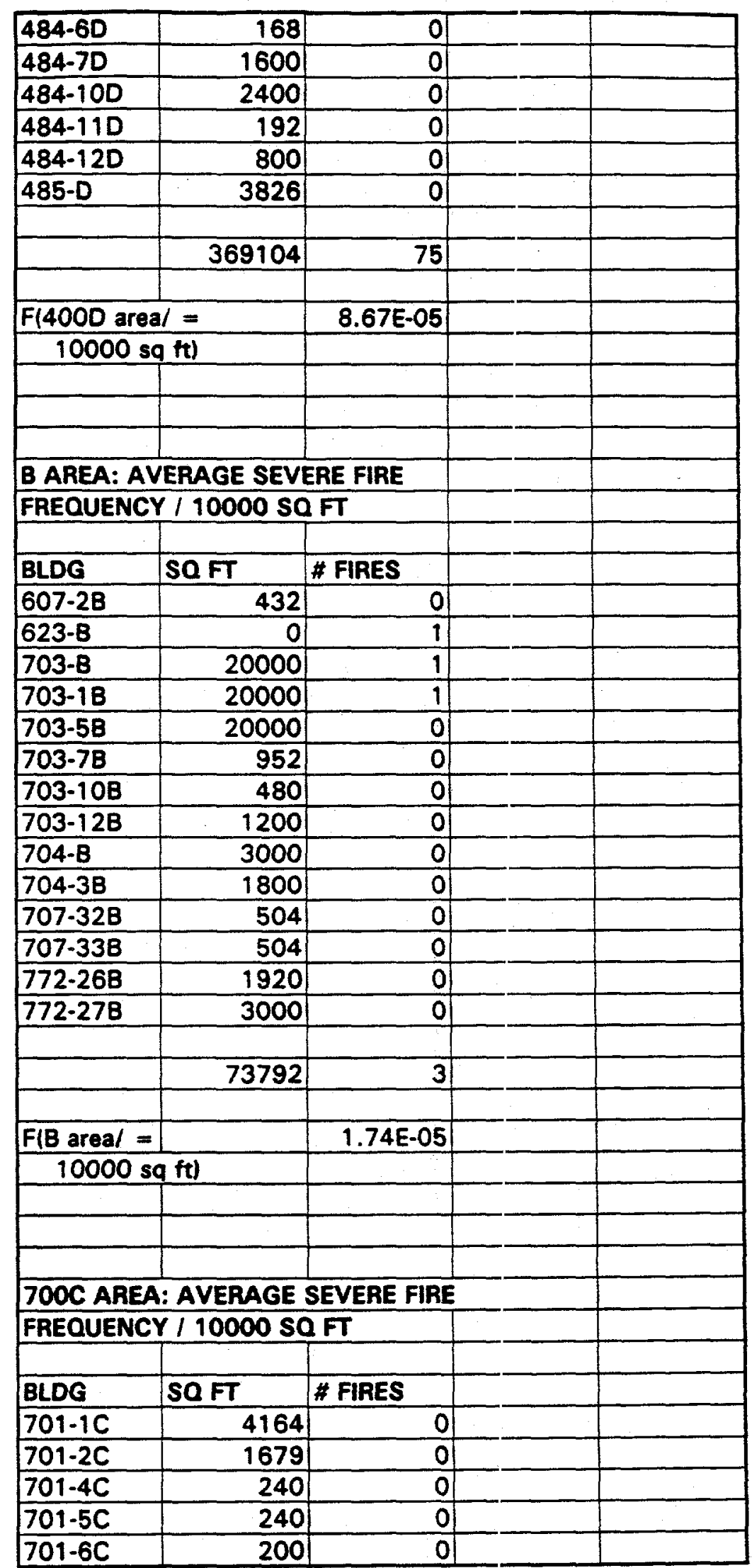


Table 2

\begin{tabular}{|c|c|c|c|c|}
\hline 702-C & 1665 & 0) & & \\
\hline 704-C & 18489 & 4 & & \\
\hline $704-1 \mathrm{C}$ & 1493 & 0 & & \\
\hline $704-2 C$ & 1792 & 0 & & \\
\hline $705-C$ & 26250 & 0 & & \\
\hline $706-C$ & 19000 & 0 & & \\
\hline $706-6 \mathrm{C}$ & 720 & 의 & & \\
\hline $706-17 \mathrm{C}$ & 1792 & o & & \\
\hline $707-C$ & 44728 & 0 & & \\
\hline $707-2 \mathrm{C}$ & 540 & o & & \\
\hline $707-3 \mathrm{C}$ & 540 & 0 & & \\
\hline $707-4 C$ & 540 & 0 & & \\
\hline $707.5 \mathrm{C}$ & 540 & 0 & & \\
\hline \multirow[t]{3}{*}{$717-C$} & 4250 & 1 & & \\
\hline & & & & \\
\hline & 128862 & 5 & & \\
\hline 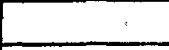 & & & & \\
\hline \multicolumn{2}{|c|}{ Fi700C areal $=$} & $1.66 \mathrm{E}-05$ & & \\
\hline \multicolumn{2}{|c|}{$10000 \mathrm{sq}(\mathrm{ft}$} & & & \\
\hline & & & & \\
\hline & & & & \\
\hline \multirow{2}{*}{\multicolumn{3}{|c|}{\begin{tabular}{l|} 
7000 AREA: AVERAGE SEVERE FIRE \\
FREQUENCY / 10000 SQ FT
\end{tabular}}} & & \\
\hline & & & & \\
\hline \multirow{2}{*}{ BLDG } & & & & \\
\hline & SQFT & \# FIRES & & \\
\hline $701-10$ & 4034 & 0 & & \\
\hline 701.20 & 100 & 0 & & \\
\hline $701 \cdot 30$ & 202 & 0. & & \\
\hline 704-D & 10087 & 1 & & \\
\hline $704-1 D$ & 1792 & 0 & & \\
\hline 707.0 & 1665 & 1 & & \\
\hline $711-D$ & 680 & 0 & & \\
\hline $711-10$ & 3646 & 0 & & \\
\hline 717-D & 18365 & 1 & & \\
\hline \multirow[t]{3}{*}{$772-D$} & 11750 & 2 & & \\
\hline & & & & \\
\hline & 52321 & 5 & & \\
\hline & & & & \\
\hline \multirow{2}{*}{\multicolumn{2}{|c|}{$\frac{F(7000 \text { area/ }=}{10000 \mathrm{sq} f t)}$}} & $4.08 \mathrm{E}-05$ & & \\
\hline & & & & \\
\hline & & & & \\
\hline & & & & \\
\hline & & & & \\
\hline \multirow{2}{*}{\multicolumn{3}{|c|}{\begin{tabular}{|l} 
G AREA: AVERAGE SEVERE FIRE \\
FREQUENCY / 10000 SQ FT \\
\end{tabular}}} & & \\
\hline & & & & \\
\hline & & & & \\
\hline BLDG & SQFT & \# FIRES & & \\
\hline
\end{tabular}


Table 2

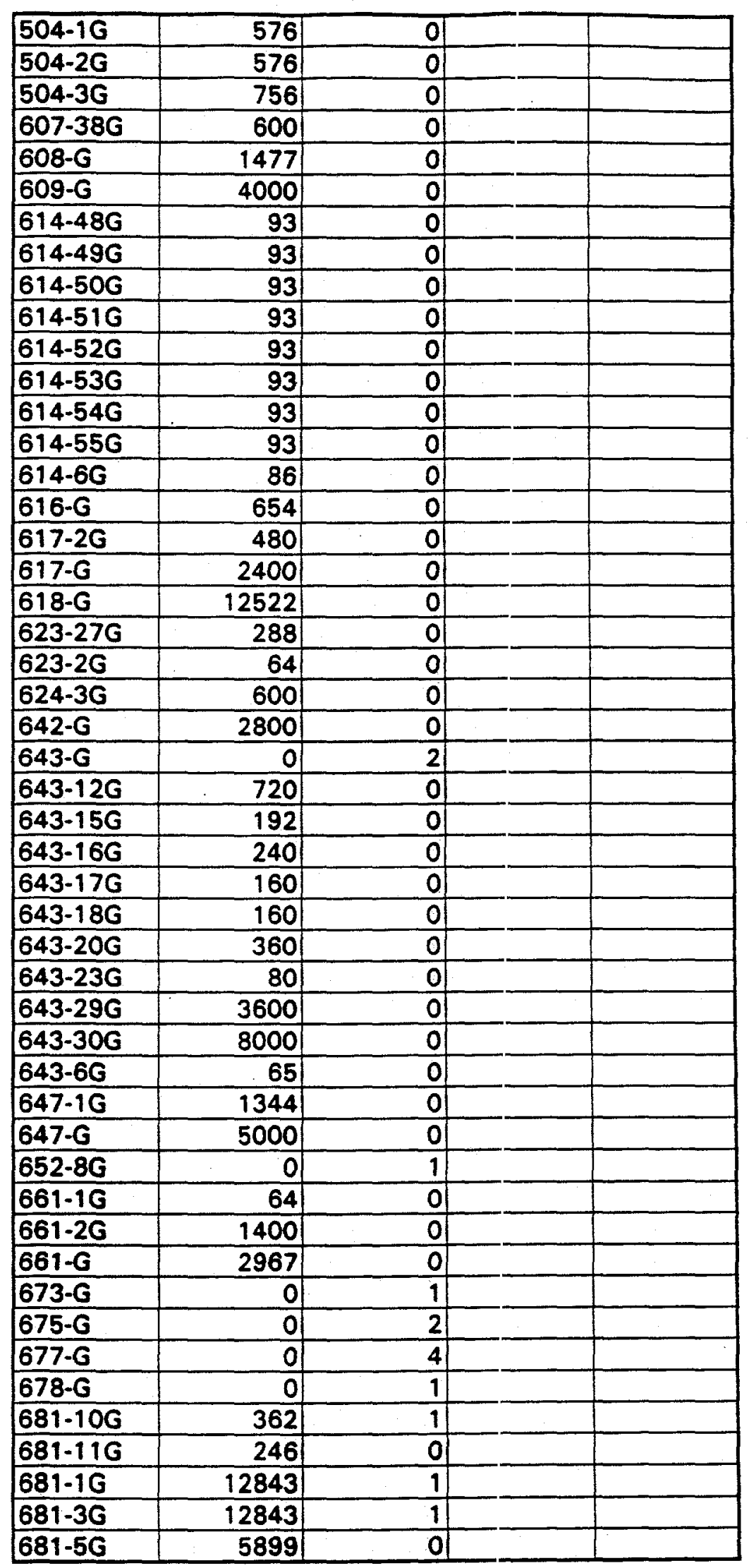


Table 2

\begin{tabular}{|c|c|c|c|c|}
\hline $681-6 \mathrm{G}$ & 2400 & 0 & & \\
\hline $681.7 \mathrm{G}$ & 1790 & 0 & & \\
\hline $681-8 \mathrm{G}$ & 400 & 0 & & \\
\hline $681-9 \mathrm{G}$ & 480 & 0 & & \\
\hline $686-3 G$ & 720 & 0 & & \\
\hline 690-G & 11500 & 3 & & \\
\hline $701-12 \mathrm{G}$ & 150 & 0 & & \\
\hline $701-13 G$ & 150 & 0 & & \\
\hline $701-146$ & 48 & 0 & & \\
\hline $701-15 \mathrm{G}$ & 48 & 0 & & \\
\hline $701-16 G$ & 48 & 0 & & \\
\hline $701-2 \mathrm{G}$ & 146 & 0 & & \\
\hline $701-3 G$ & 146 & 0 & & \\
\hline $701-4 G$ & 146 & 0 & & \\
\hline $701-5 G$ & 146 & 0 & & \\
\hline $701-6 \mathrm{G}$ & 146 & 0 & & \\
\hline $701-8 \mathrm{G}$ & 150 & 0 & & \\
\hline $703-5 \mathrm{G}$ & 2700 & 0 & & \\
\hline $703-6 \mathrm{G}$ & 5600 & 0 & & \\
\hline $703.7 \mathrm{G}$ & 240 & 0 & & \\
\hline $703-9 \mathrm{G}$ & 200 & 0 & & \\
\hline $704-1 \mathrm{G}$ & 980 & 0 & & \\
\hline $704-2 G$ & 784 & 0 & & \\
\hline $704-3 G$ & 980 & 0 & & \\
\hline $704-4 \mathrm{G}$ & 460 & 0 & & \\
\hline $705-G$ & 11926 & 1 & & \\
\hline $706-1 \mathrm{G}$ & 200 & 0 & & \\
\hline $706-3 G$ & 10500 & 0 & & \\
\hline $706-\mathrm{G}$ & 7333 & 0 & & \\
\hline $707-G$ & 961 & 0 & & \\
\hline $709-1 \mathrm{G}$ & 750 & of & & \\
\hline $709-2 \mathrm{G}$ & 13550 & o) & & \\
\hline $709-4 G$ & 11550 & 0 & & \\
\hline $709-G$ & 10080 & 0 & & \\
\hline $710-2 \mathrm{G}$ & 800 & 0 & & \\
\hline $710-G$ & 15360 & 1 & & \\
\hline $711-\mathrm{G}$ & 6000 & 0 & & \\
\hline $714-2 \mathrm{G}$ & 11956 & 0 & & \\
\hline $714-5 G$ & 18670 & 0 & & \\
\hline $714-6 G$ & 16000 & of & & \\
\hline $714-7 G$ & 16000 & 0 & & \\
\hline $714-G$ & 8000 & 0 & & \\
\hline $715-1 \mathrm{G}$ & 576 & o & & \\
\hline $715-\mathrm{G}$ & 140 & 0 & & \\
\hline $717-G$ & 3244 & 0 & & \\
\hline $722-G$ & 6030 & o & & \\
\hline $724-7 \mathrm{G}$ & 5231 & 1 & & \\
\hline $724-8 G$ & 1300 & 0 & & \\
\hline $726-1 \mathrm{G}$ & 1600 & 0 & & \\
\hline
\end{tabular}


Table 2

\begin{tabular}{|c|c|c|c|c|}
\hline $728-G$ & 1201 & 0 & & \\
\hline $730-G$ & 8080 & of & & \\
\hline $734-G$ & 308 & 0) & & \\
\hline $735-7 \mathrm{G}$ & 4920 & 0 & & \\
\hline $735-7 \mathrm{G}$ & 1550 & 0 & & \\
\hline $739-1 \mathrm{G}$ & 200 & of & & \\
\hline $739-4 \mathrm{G}$ & 360 & 0 & & \\
\hline $739-6 \mathrm{G}$ & 48 & 0 & & \\
\hline $741-1 \mathrm{G}$ & 400 & of & & \\
\hline $741-2 G$ & 8000 & 0 & & \\
\hline $741-G$ & 600 & 0 & & \\
\hline $760-10 G$ & 144 & 0 & & \\
\hline $760-11 \mathrm{G}$ & 0 & 1 & & \\
\hline $760-12 \mathrm{G}$ & 1500 & 0 & & \\
\hline $760-13 G$ & 2000 & 0 & & \\
\hline $760-1 \mathrm{G}$ & 4138 & 1 & & \\
\hline $760-2 \mathrm{G}$ & 714 & 0 & & \\
\hline $760-3 G$ & 3024 & 0 & & \\
\hline $760-4 G$ & 3760 & o) & & \\
\hline $760-5 \mathrm{G}$ & 1792 & 0 & & \\
\hline $760-8 G$ & 865 & 0 & - & \\
\hline $760-9 \mathrm{G}$ & 3600 & 0 & & \\
\hline $760-G$ & 4074 & 0 & & \\
\hline $772-10 \mathrm{G}$ & 7800 & 0 & & \\
\hline $772-13 G$ & 720 & 0 & & \\
\hline $772-14 G$ & 672 & 0 & & \\
\hline $772-17 \mathrm{G}$ & 768 & 0 & & \\
\hline $772-18 \mathrm{G}$ & 660 & 0 & & \\
\hline $772-19 \mathrm{G}$ & 660 & 0 & & \\
\hline $772-1 \mathrm{G}$ & 7786 & 0 & & \\
\hline $772-2 G$ & 0 & 1 & & \\
\hline $772.20 \mathrm{G}$ & 1000 & 0 & & \\
\hline $772.21 \mathrm{G}$ & 648 & 0 & & \\
\hline $772-22 \mathrm{G}$ & 648 & 0 & & \\
\hline $772-25 \mathrm{G}$ & 2400 & 0 & & \\
\hline $772.26 G$ & 200 & 0 & & \\
\hline $772.3 \mathrm{G}$ & 240 & 0 & & \\
\hline $772-5 \mathrm{G}$ & 668 & 0 & & \\
\hline $772-7 \mathrm{G}$ & 8000 & 0 & & \\
\hline $772-8 \mathrm{G}$ & 7800 & 0 & & \\
\hline $772.9 \mathrm{G}$ & 7800 & 0 & & \\
\hline $772-G$ & 7695 & 0 & & \\
\hline $904-116 \mathrm{G}$ & 48 & 0 & & \\
\hline $905-109 \mathrm{G}$ & 48 & 0 & & \\
\hline $905-10 \mathrm{G}$ & 48 & 0 & & \\
\hline $905-110 G$ & 48 & 0 & & \\
\hline $905-111 \mathrm{G}$ & 48 & 0 & & \\
\hline $905-115 \mathrm{G}$ & 48 & 0 & & \\
\hline $905-11 \mathrm{G}$ & 36 & 0 & & \\
\hline
\end{tabular}


Table 2

\begin{tabular}{|c|c|c|c|c|}
\hline $905-6 \mathrm{G}$ & 48 & of & & \\
\hline $905-74 G$ & 36 & of & & \\
\hline 905-7G & 48 & of & & \\
\hline $905-8 \mathrm{G}$ & 48 & of & & \\
\hline $725-1 N$ & 4500 & 0 & & \\
\hline $717 \cdot 11 \mathrm{~N}$ & 5265 & 0 & & \\
\hline $711-\mathrm{N}$ & 45600 & 0 & & \\
\hline $713-1 \mathrm{~N}$ & 30000 & 0 & & \\
\hline $713-N$ & 30000 & 0 & & \\
\hline $716-N$ & 15850 & 0 & & \\
\hline $717-10 \mathrm{~N}$ & 12000 & 2 & & \\
\hline $717-5 N$ & 22000 & 0 & & \\
\hline $717-8 \mathrm{~N}$ & 7400 & 0 & & \\
\hline \multirow[t]{2}{*}{$719-N$} & 6500 & 0 & & \\
\hline & 580545 & 25 & & \\
\hline & 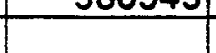 & & & \\
\hline FIG area/ $=$ & & $1.84 E-05$ & & \\
\hline \multicolumn{5}{|c|}{$10000 \mathrm{sq} f t)$} \\
\hline & & & & \\
\hline & & & & \\
\hline \multicolumn{5}{|c|}{ TNX AREA: AVERAGE SEVERE FIRE } \\
\hline \multicolumn{5}{|c|}{ FREQUENCY / 10000 SQ FT } \\
\hline & & & & \\
\hline BLDG & SQ FT & \# FIRES & & \\
\hline $607-41 \mathrm{~T}$ & 400 & 0 & & \\
\hline $607-46 \mathrm{~T}$ & 600 & 0 & & \\
\hline $669-T$ & 3150 & 0 & & \\
\hline 670-T & 3600 & 0 & & \\
\hline $671-T$ & 200 & 0 & & \\
\hline 672-T & 12120 & 0 & & \\
\hline 673-T & 4800 & 0 & & \\
\hline 674-T & 7000 & 0 & & \\
\hline 675-T & 6642 & 1 & & \\
\hline 676-T & 1536 & 0 & & \\
\hline $676-1 T$ & 1536 & 0 & & \\
\hline 676-2T & 720 & 0 & & \\
\hline $676-8 \mathrm{~T}$ & 1536 & 0 & & \\
\hline $676-9 T$ & 1536 & 0 & & \\
\hline $676-10 \mathrm{~T}$ & 1536 & 0 & & \\
\hline $676-11 T$ & 1536 & 0 & & \\
\hline $676-12 \mathrm{~T}$ & 1536 & 0 & & \\
\hline $676-14 \mathrm{~T}$ & 1792 & 0 & & \\
\hline $676-15 \mathrm{~T}$ & 1792 & 0 & & \\
\hline $677-\mathrm{T}$ & 15280 & 0 & & \\
\hline $677-1 \mathrm{~T}$ & 192 & 0 & & \\
\hline $677-2 T$ & 192 & 0 & & \\
\hline 678-T & 12957 & 4 & & \\
\hline
\end{tabular}


Table 2

\begin{tabular}{|c|c|c|c|c|}
\hline 679-T & 13360 & of & & \\
\hline $679-2 T$ & 120 & 0 & & \\
\hline $679-5 T$ & 48 & of & & \\
\hline $679-7 \mathrm{~T}$ & 101 & of & & \\
\hline $679-8 \mathrm{~T}$ & 101 & of & & \\
\hline 682-T & 500 & 0 & & \\
\hline 684-T & 420 & 의 & & \\
\hline 704-T & 6395 & 0 & & \\
\hline $704-1 \mathrm{~T}$ & 6500 & of & & \\
\hline $711-T$ & 200 & of & & \\
\hline $772-T$ & 40000 & 1 & & \\
\hline 904-T & 3600 & 0 & & \\
\hline & & & & \\
\hline & 153534 & 6 & & \\
\hline \multirow{2}{*}{\multicolumn{2}{|c|}{ F(TNX area/ $=$}} & & & \\
\hline & & $1.67 E-05$ & & \\
\hline \multicolumn{5}{|c|}{$10000 \mathrm{sq} f \mathrm{ft}$} \\
\hline & & & & \\
\hline & & & & \\
\hline & & & & \\
\hline \multicolumn{5}{|c|}{ 700K AREA: AVERAGE SEVERE FIRE } \\
\hline \multicolumn{5}{|c|}{ FREQUENCY / 10000 SQ FT } \\
\hline & & & & \\
\hline BLDG & SQFT & \# FIRES & & \\
\hline $701-1 \mathrm{~K}$ & 4162 & $1]$ & & \\
\hline $701-2 \mathrm{~K}$ & 1679 & 0] & & \\
\hline $701-5 K$ & 308 & 0) & & \\
\hline $704-K$ & 15870 & of & & \\
\hline $704-1 \mathrm{~K}$ & 1792 & 의 & & \\
\hline $704-2 K$ & 845 & 0 & & \\
\hline $704-3 K$ & 845 & of & & \\
\hline $704-4 K$ & 845 & O) & & \\
\hline $711-1 \mathrm{~K}$ & 800 & of & & \\
\hline \multirow[t]{3}{*}{$717-K$} & 20000 & of & & \\
\hline & & & & \\
\hline & 47146 & 1 & & \\
\hline \multicolumn{2}{|c|}{ F(700K areal $=$} & $905 \mathrm{~F}-06$ & & \\
\hline \multicolumn{5}{|c|}{$10000 \mathrm{sq} f t$} \\
\hline & & & & \\
\hline & & & & \\
\hline & & & & \\
\hline & & & & \\
\hline $700 \mathrm{~L}$ AF & AVERAGE S & SEVERE FIRE & & \\
\hline FREQUE & $110000 \mathrm{s0}$ & FT & & \\
\hline & & & & \\
\hline BLDG & SQFT & \# FIRES & & \\
\hline
\end{tabular}


Table 2

\begin{tabular}{|c|c|c|c|c|}
\hline $701-1 \mathrm{~L}$ & 228 & 0 & & \\
\hline $701-2 L$ & 1679 & 2 & & \\
\hline $701-5 \mathrm{~L}$ & 308 & 0 & & \\
\hline $704-L$ & 14487 & 1 & & \\
\hline $704-1 \mathrm{~L}$ & 1792 & 0 & & \\
\hline $711-L$ & 630 & of & & \\
\hline $717-L$ & 0 & 1 & & \\
\hline $723-L$ & 180 & 0 & & \\
\hline $723-1 L$ & 85 & 0 & & \\
\hline $723-2 \mathrm{~L}$ & 80 & 0 & & \\
\hline $723-3 L$ & 80 & 0 & & \\
\hline $723-4 \mathrm{~L}$ & 200 & 0 & & \\
\hline & & & & \\
\hline & 19749 & 4 & & \\
\hline$F 17001$ ar & & $8.64 E-05$ & & \\
\hline 10000 & ft) & & & \\
\hline & & & & \\
\hline & & & & \\
\hline & & & & \\
\hline $700 M$ AR & AVERAGE & SEVERE FIRE & & \\
\hline FREQUEN & $1110000 \mathrm{s0}$ & & & \\
\hline BLDG & SOFT & \# FIRES & & \\
\hline $701-1 M$ & 315 & 0 & & \\
\hline $701-3 M$ & 195 & 0 & & \\
\hline $701-4 M$ & 192 & 1 & & \\
\hline $701-6 M$ & 0 & 1 & & \\
\hline $701.7 M$ & 420 & 0 & & \\
\hline 704-M & 7672 & of & & \\
\hline $710-M$ & 250 & 0 & & \\
\hline $730-M$ & 18000 & 0 & & \\
\hline $734-M$ & 450 & 0 & & \\
\hline $777-M$ & 0 & 3 & & \\
\hline $782-1 M$ & 320 & 0 & & \\
\hline & & & & \\
\hline & 27814 & 5 & & \\
\hline & & & & \\
\hline $\mathrm{F1700Ma}$ & $a=$ & $7.67 \mathrm{E}-05$ & & \\
\hline 10000 & $\mathrm{ft}$ & & & \\
\hline & & & & \\
\hline & & & & \\
\hline & & & & \\
\hline DOP AR: & AVERAGE $S$ & EVERE FIRE & & \\
\hline FREQUEN & $1 / 1000050$ & & & \\
\hline$B$ BLI & 30 & \# FIRE & & \\
\hline
\end{tabular}


Table 2

\begin{tabular}{|c|c|c|c|c|}
\hline $701-1 P$ & 4164 & 0 & & \\
\hline $701-2 P$ & 1679 & 1 & & \\
\hline $701-5 P$ & 308 & of & & \\
\hline 704-P & 15870 & 2 & & \\
\hline $704-2 P$ & 870 & 0 & & \\
\hline $704-3 P$ & 896 & 0 & & \\
\hline \multirow{3}{*}{$711 . P$} & 630 & 0 & & \\
\hline & 24417 & 3 & & \\
\hline & & & & \\
\hline \multicolumn{2}{|c|}{ F(700P areal $=$} & $5.24 E-05$ & & \\
\hline \multicolumn{2}{|c|}{$10000 \mathrm{sq} f t$} & & & \\
\hline \multirow{2}{*}{\multicolumn{3}{|c|}{\begin{tabular}{|l|} 
700R AREA: AVERAGE SEVERE FIRE \\
FREQUENCY / 10000 SO FT \\
\end{tabular}}} & & \\
\hline & & & & \\
\hline \multirow{9}{*}{\begin{tabular}{|l|} 
BLDG \\
$701-1 R$ \\
$701-2 R$ \\
$701-3 R$ \\
$704-R$ \\
$711-R$ \\
\\
\end{tabular}} & & & & \\
\hline & SOFT & \# FIRES & & \\
\hline & 4162 & 0 & & \\
\hline & 150 & 0 & & \\
\hline & 64 & 0 & & \\
\hline & 15870 & 1 & & \\
\hline & 630 & 0 & & \\
\hline & & & & \\
\hline & 20876 & 1 & & \\
\hline \multirow{2}{*}{\multicolumn{2}{|c|}{$\begin{array}{r}F(700 \mathrm{R} \text { area/ }= \\
10000 \mathrm{sq} \mathrm{ft}) \\
\end{array}$}} & 2.04E-05 & & \\
\hline & & & & \\
\hline & & & & \\
\hline & & & & \\
\hline & & & & \\
\hline & & & & \\
\hline & & & & \\
\hline & & & & \\
\hline & & & & \\
\hline & & & & \\
\hline & & & & \\
\hline & & & & \\
\hline & & & & \\
\hline & & & & \\
\hline
\end{tabular}

